\title{
Progression in Non-Destructive Spallation Prediction and Elevated Temperature Mechanical Property Evaluation of Thermal Barrier Coating Systems by Use of a Spherical Micro-Indentation Method
}

Jared Michael Tannenbaum

West Virginia University

Follow this and additional works at: https://researchrepository.wvu.edu/etd

\section{Recommended Citation}

Tannenbaum, Jared Michael, "Progression in Non-Destructive Spallation Prediction and Elevated Temperature Mechanical Property Evaluation of Thermal Barrier Coating Systems by Use of a Spherical Micro-Indentation Method" (2011). Graduate Theses, Dissertations, and Problem Reports. 3054. https://researchrepository.wvu.edu/etd/3054

This Dissertation is protected by copyright and/or related rights. It has been brought to you by the The Research Repository @ WVU with permission from the rights-holder(s). You are free to use this Dissertation in any way that is permitted by the copyright and related rights legislation that applies to your use. For other uses you must obtain permission from the rights-holder(s) directly, unless additional rights are indicated by a Creative Commons license in the record and/ or on the work itself. This Dissertation has been accepted for inclusion in WVU Graduate Theses, Dissertations, and Problem Reports collection by an authorized administrator of The Research Repository @ WVU. For more information, please contact researchrepository@mail.wvu.edu. 


\title{
Progression in Non-Destructive Spallation Prediction and Elevated Temperature Mechanical Property Evaluation of Thermal Barrier Coating Systems by Use of a Spherical Micro-Indentation Method
}

\author{
Jared Michael Tannenbaum
}

Dissertation submitted to the

College of Engineering and Mineral Resources

at West Virginia University

in partial fulfillment of the requirements

for the degree of

Doctor of Philosophy

In

Mechanical Engineering

Bruce Kang, Ph.D. Committee Chairman

Marry Anne Alvin

Gary Morris, Ph.D.

Victor Mucino, Ph.D.

Edward Sabolsky, Ph.D.

Nianqiang Wu, Ph.D.

Department of Mechanical and Aerospace Engineering

Morgantown, West Virginia

2011

Keywords: Thermal barrier coating; load-depth sensing indentation; elevated temperature material characterization 


\section{ABSTRACT}

\section{Progression in Non-Destructive Spallation Prediction and Elevated Temperature Mechanical Property Evaluation of Thermal Barrier Coating System by Use of a Spherical Micro-Indentation Method}

Jared M. Tannenbaum

The accumulation of carbon dioxide in the atmosphere continues to be an important ecological issue associated with global warming. The demand for improved efficiencies in energy conversion in recent years has led to the introduction of land-based gas turbines with significantly increased inlet temperatures. To accomplish this, nickel-based superalloys with protective thermal barrier coatings (TBC) are widely used as systems capable of extending the life and increasing firing temperatures of combustor and stationary turbine components. However, coating durability, thermal-fatigue and erratic spallation failure currently limit the continuous operation of turbine engines. Of the present ceramic coating materials used, yttria stabilized zirconia (YSZ) is most prevalent; its low thermal conductivity, high thermal expansion coefficient and outstanding mechanical strength make it ideal for use in TBC systems. However, residual stresses caused by coefficients of thermal expansion mismatches within the TBC system and unstable thermally-grown oxides (TGO) are considered the primary causes for its premature and erratic spallation failure. The development of new materials, coating technologies and evaluation techniques is required if enhanced efficiency is to be achieved. As a result, several non-destructive evaluation (NDE) techniques have been developed to address this problem yet few comprehensive studies have resulted in the development of true NDE techniques capable of predicting failure location prior to its occurrence.

In this research, a load-based micro-indentation method for NDE of TBCs exposed to thermal loads in air has been developed. Coating surface stiffness responses obtained through use of this technique have been found capable of assessing damage accumulation and macroscopic debonding failure sites following thermal exposure of TBC systems to elevated temperatures. Furthermore, microstructural analyses correlating these surface stiffness response to overall YSZ/TGO interface conditions indicate that high interfacial rumpling and non-uniform oxide growth leads to the development of both in-plane and out-of-plane residual stresses. As a result, areas displaying relative increases in surface stiffness response enable early detection of initial TBC spallation locations. Additionally, with the evolution of nanotechnologies, indentation testing techniques have become more common, yet their ability to evaluate material mechanical properties in harsh environments remains a challenge. Following a classical Hertzian contact mechanics approach, a microindentation technique that does not require system compliance calibration or the use of high precision depth sensors has been developed. The removal of these constraints has led to the development of both a portable and high-temperature micro-indentation system for TBC materials mechanical property evaluation up to $1000^{\circ} \mathrm{C}$. 


\section{Table of Contents}

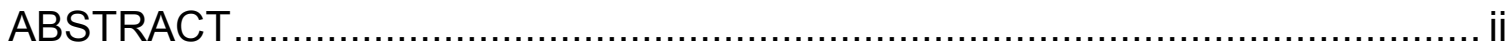

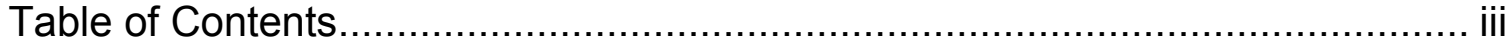

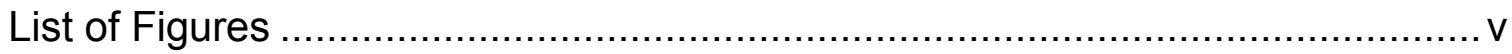

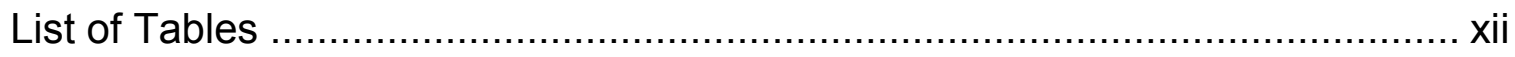

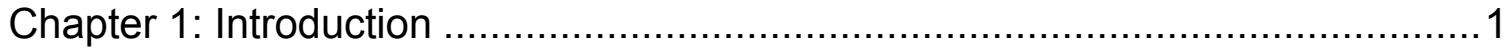

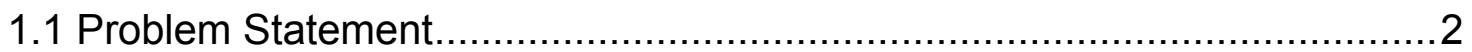

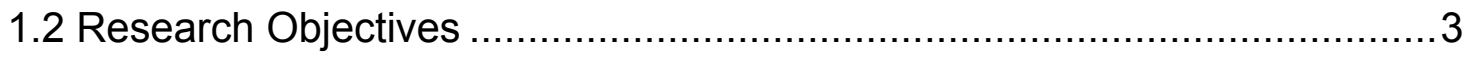

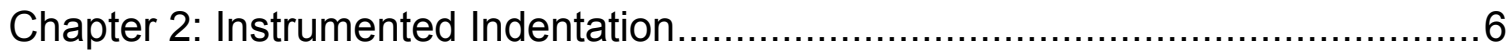

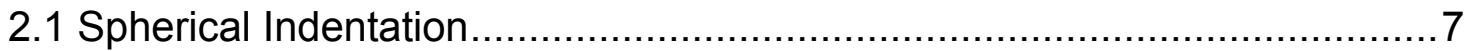

2.2 Instrumented Indentation Data Analysis ..................................................... 9

2.2.1 Single Point Unloading .............................................................. 10

2.2.2 Multiple Partial Unloading .......................................................11

2.3 Instrumented Indentation Systems …............................................... 14

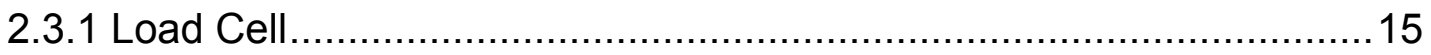

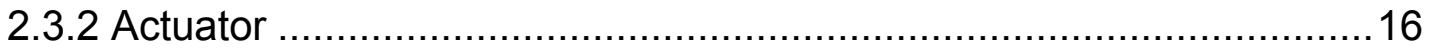

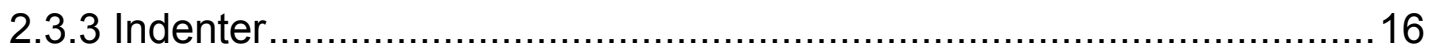

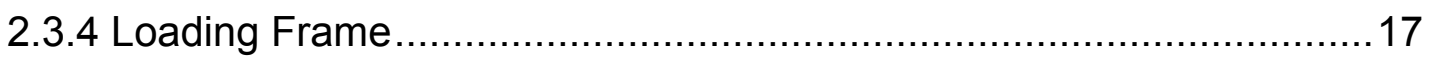

2.4 Factors Affecting Instrumented Indentation ........................................ 17

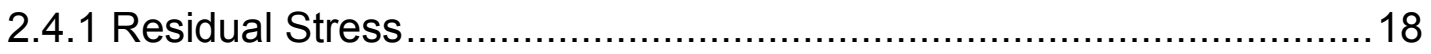

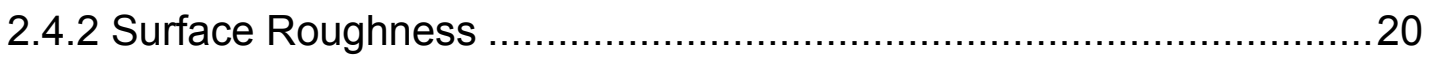

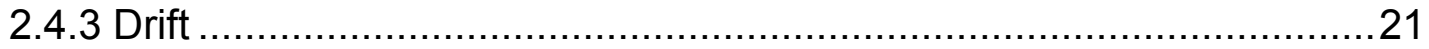

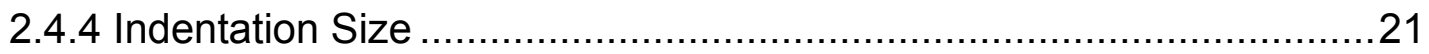

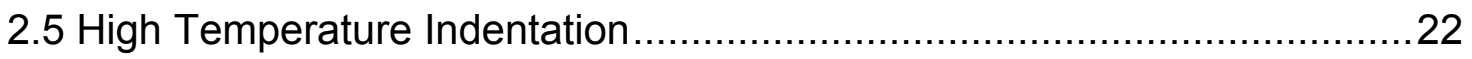

2.5.1 System Development and Description ...........................................24

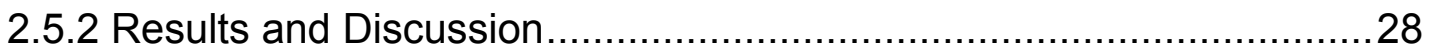

2.6 Environmental High Temperature Indentation .......................................31

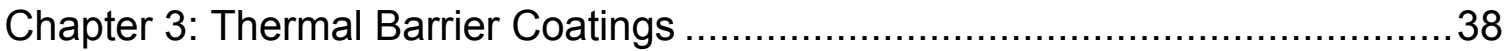

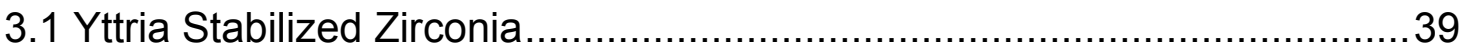

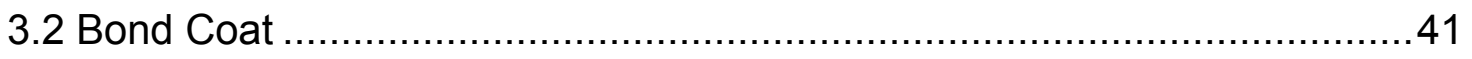

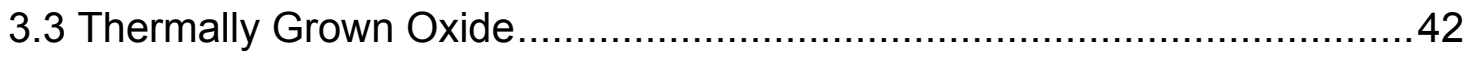




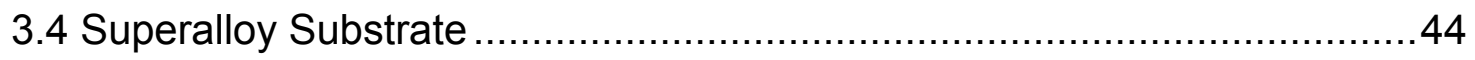

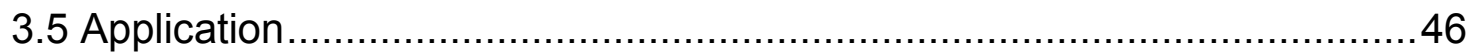

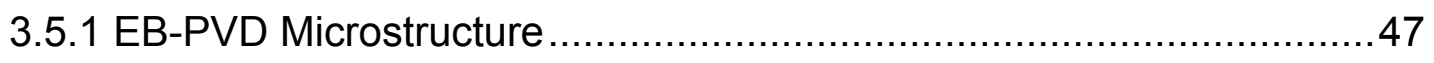

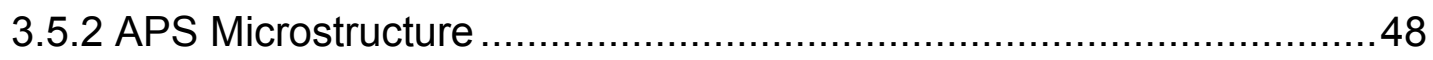

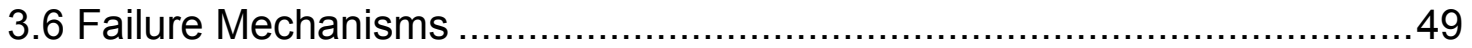

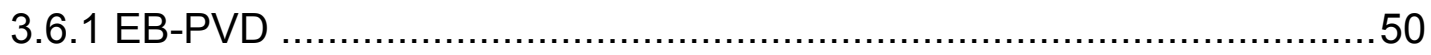

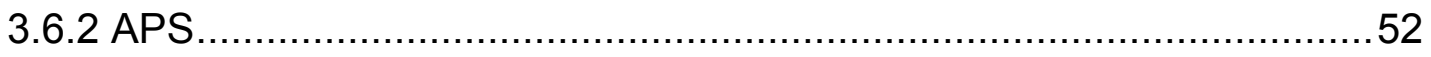

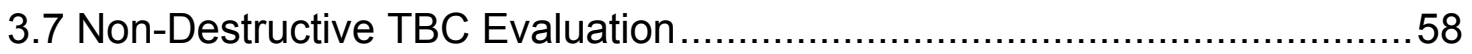

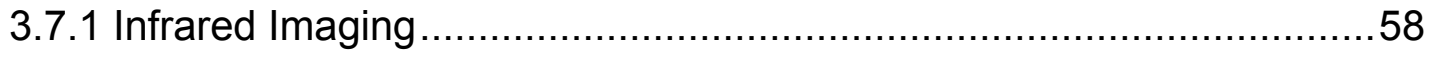

3.7.2 Photoluminescence Piezospectroscopy ........................................60

3.7.3 Electrochemical Impedance Spectroscopy ....................................62

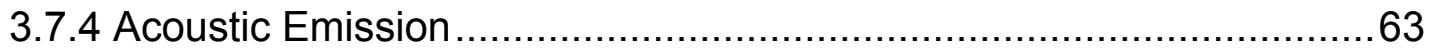

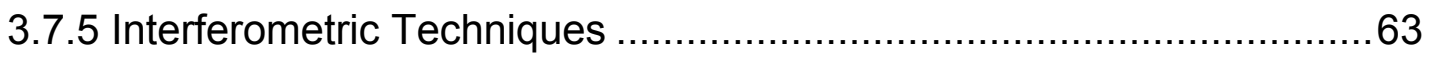

3.8 Load Based Micro-Indentation Evaluation ...............................................64

3.8.1 Thermal Barrier Coating Coupons ................................................65

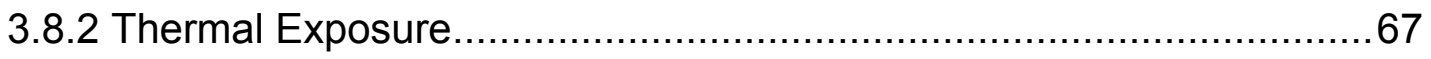

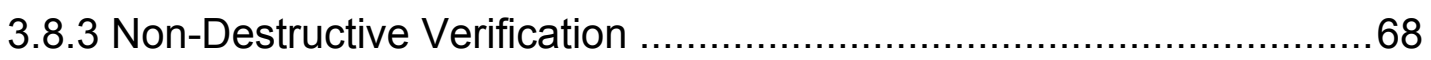

3.8.4 Unloading Slope Measurement Results ........................................70

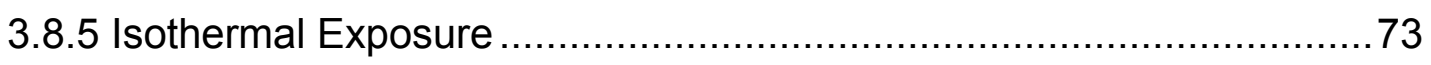

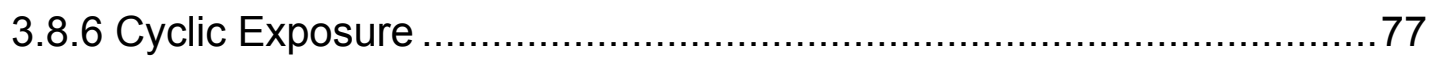

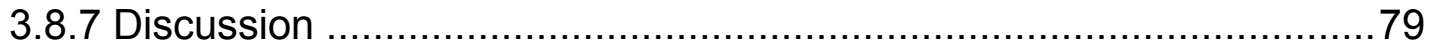

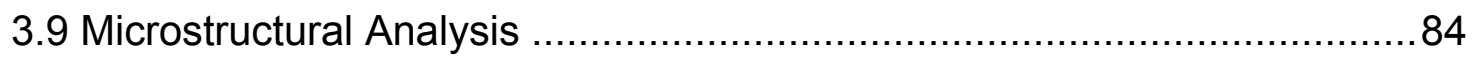

3.9.1 Object Oriented Finite Element Method ......................................... 85

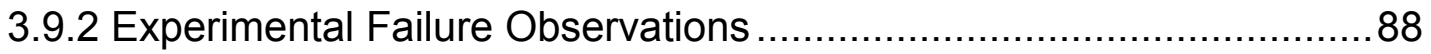

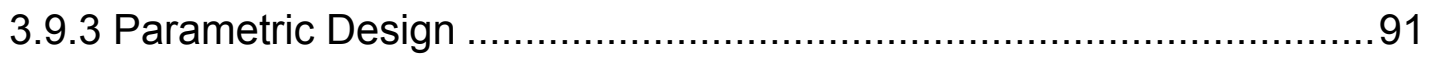

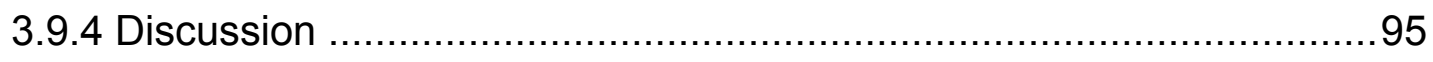

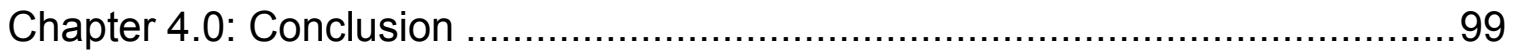

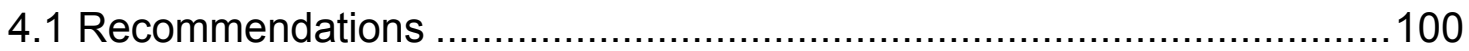

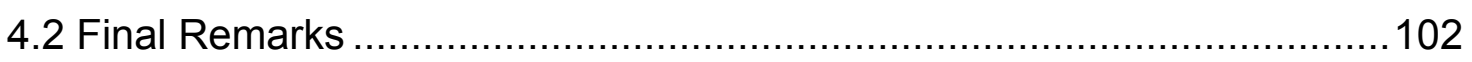

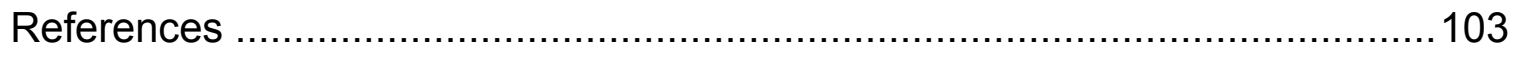

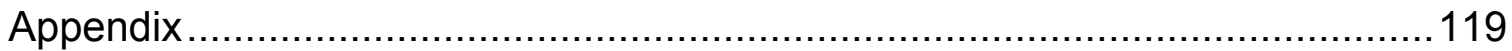




\section{List of Figures}

Figure 1: Contact deformation between two spheres [10].

Figure 2: Pile-up and sink-in (not simultaneously) occurring during spherical

indentation.

Figure 3: Load displacement curve for single point unloading algorithm............10

Figure 4: Load displacement curve for multiple partial unloading algorithm........12

Figure 5: Instrumented indentation system loading chain schematic................. 15

Figure 6: Elastic modulus variance with an imposed residuals stress [23].........19

Figure 7: Atomic force micrograph of a $0.76-\mathrm{mm}$ thick aluminum film [32] .........20

Figure 8: Micro-indentation probe for measuring plastic deformation of porous thermal barrier oxides at temperatures between $937^{\circ} \mathrm{C}$ and $1137^{\circ} \mathrm{C}$ [37].

Figure 9: Instrumented micro-indentation equipment for experiments up to $1000^{\circ} \mathrm{C}$ [38].

Figure 10: High temperature indentation system loading frame design; a) i: glass fiber insulation, ii: furnace heating element, iii: sample, iv: spherical sapphire $750 \mu \mathrm{m}$ radius indenter, v: alumina indenter rod, vi: $0.8 \mathrm{~nm}$ resolution Physik Instrumente P-246.7S $120 \mu \mathrm{m}$ piezoelectric actuator, vii: $\pm 0.1 \%$ accuracy Honeywell Model $75100 \mathrm{lb}$. load cell and b) optical image. 25

Figure 11: High temperature indentation system design, a) room temperature and b) high temperature. 26

Figure 12: High temperature indentation elastic modulus results on an $\mathrm{H} 13$ tool steel coupon with \pm 1 standard deviation [43].

Figure 13: High temperature indentation elastic modulus results on a Haynes 230 coupon with \pm 1 standard deviation [44]. 30

Figure 14: Iron oxide scales grown on $\mathrm{H} 13$ Tool Steel at $500^{\circ} \mathrm{C}$; a) x250 and b) $\mathrm{x} 1700$ 30

Figure 15: Controlled environment high temperature indentation system. 
Figure 16: Controlled environment high temperature indentation system schematic; i) stainless steel bellows, ii) gas/moisture/vacuum port, iii) sample (shown schematically in Figure 11) and iv) cylindrical alumina tube. 33

Figure 17: Controlled environment high temperature indentation system, gas/steam and water cooling lines.

Figure 18: Controlled environment high temperature indentation system, external water pumps and cooling tank. 35

Figure 19: Controlled environment high temperature indentation system;

overview. 36

Figure 20: Controlled gas high temperature indentation system at a) room temperature and b) $1100^{\circ} \mathrm{C}$. 37

Figure 21: Yttria stabilized zirconia phase diagram [53] 39

Figure 22: Reduction in relative coating durability with increasing thickness [56].

Figure 23: Thermally grown oxide microstructure [52] ............................... 43

Figure 24: TBC Cross-sectional SEM Micrograph, a) EB-PVD and b) APS ........46

Figure 25: EB-PVD columnar structure formed upon application [74] ................47

Figure 26: Plasma spray splat impact formation [75] ..................................48

Figure 27: Plasma spray splat microstructure [77] .................................... 49

Figure 28: EB-PVD TBC cross-sectional scanning electron micrographs of Ptaluminide bond coats showing the imperfections that enlarge by ratcheting. [86].

Figure 29: Schematic diagram and scanning electron micrograph showing the four different cracking mechanisms of a failed APS TBC after 240 cycles [93].

Figure 30: Cross-sectional scanning electron micrograph of an APS TBC system thermal load at $1070^{\circ} \mathrm{C}$ in air after a) 95 and b) 585 hours [94] ........54

Figure 31: APS TBC system micro-crack development [97] ............................54

Figure 32: Oxide scale thickness induced stress analysis [98] .......................55

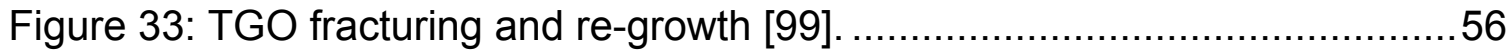




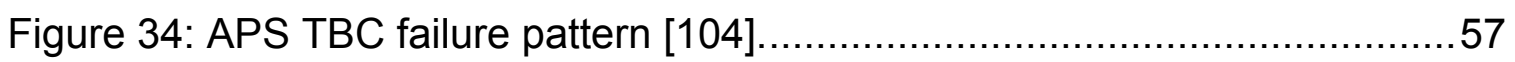

Figure 35: YSZ residual stresses measured by XRD techniques [106] ..............57

Figure 36: Delamination indication by a) white light and $b$ ) mid-infrared imaging [116]. 60

Figure 37: Experimental setup, i) $\pm 0.15 \%$ accuracy Honeywell Model $31100 \mathrm{lb}$. load cell, ii) $3.6 \mathrm{~nm}$ resolution Physik Instrumente P-239.9S $180 \mu \mathrm{m}$ piezoelectric actuator, iii) spherical tungsten carbide (WC) $750 \mu \mathrm{m}$ radius indenter, and iv) coupon stage. 66

Figure 38: Top coat SEM micrographs of an APS/MCrAIY/RenéN5 obtained after a cumulative 375 isothermal hours, a) Non-Indented Region and b) Indented Region. 68

Figure 39: Top coat SEM micrographs of an EB-PVD/MDL/RenéN5 obtained after a cumulative 60 cycles, a) Non-Indented Region and b) Indented Region. .68

Figure 40: Failure location along the surface of the thermally cycled EBPVD/MDL/RenéN5 coupon. 69

Figure 41: Axisymmetric numerical TBC indentation model boundary conditions and indenter radius of $793.5 \mu \mathrm{m}$.

Figure 42: $90 \mathrm{~N}$ indentation inference zone shown to exist in all layers of the TBC system, Von Mises Stress (MPa)...............................................70

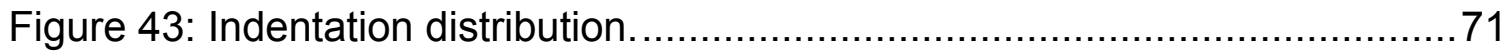

Figure 44: Outliers unloading slope measurements defined as those data points that reside within and above $80 \%$ of the first standard deviation. ......72

Figure 45: APS/MCrAIY/RenéN5 coupon A, a) surface stiffness response contour map after 450 hours of isothermal exposure at $1100^{\circ} \mathrm{C}$ and $\mathrm{b}$ ) edge spallation following 500 hours of isothermal exposure. .74

Figure 46: APS/MCrAIY/RenéN5 coupon B, a) surface stiffness response contour map after 360 hours of isothermal exposure at $1100^{\circ} \mathrm{C}$ and $b$ ) edge spallation following 400 hours of isothermal exposure. .75 
Figure 47: APS/MCrAIY/RenéN5 coupon C, a) surface stiffness response contour map after 400 hours of isothermal exposure at $1100^{\circ} \mathrm{C}$ and b) desktop edge spallation 1.5 weeks after test completion.

Figure 48: APS/MCrAIY/RenéN5 coupon D, a) surface stiffness response contour map after 360 hours of isothermal exposure at $1100^{\circ} \mathrm{C}$ and $b$ ) edge spallation following 400 hours of isothermal exposure.

Figure 49: APS/MCrAIY/RenéN5 Coupon E, a) surface stiffness response contour map after 360 thermal cycles and b) edge spallation following 400 thermal cycles.

Figure 50: APS /MCrAIY/RenéN5 Coupon F, a) surface stiffness response contour map after 400 thermal cycles and b) complete debonding of the APS YSZ along the TGO/metal interface after 440 thermal cycles.

Figure 51: APS /MCrAIY/RenéN5 Coupon G, a) surface stiffness response contour map after 400 thermal cycles and b) edge spallation following 440 thermal cycles.

Figure 52: EB-PVD/MDL/RenéN5 Coupon $\mathrm{H}$, a) surface stiffness response contour map after 600 thermal cycles and b) extensive spallation after 640 thermal cycles.

Figure 53: Average APS TBC high, low and average surface stiffness responses along TBC life percentage with $1 \sigma$ (standard deviation) shown. 80

Figure 54: Average APS TBC coefficient of variation in surface stiffness response measurement with $1 \sigma$ (standard deviation) shown.

Figure 55: Average TGO residual stress along thermal exposure acquired by PLPS methods [139].

Figure 56: EB-PVD/MDL/RenéN5 coupon edge cross sectional scanning electron micrographs after 640 isothermal cycles, a) high and b) low residually stressed region.

Figure 57: YSZ/TGO/BC microstructure geometry interface. .86

Figure 58: Object oriented finite element analysis model having a thickness of 12700 microns. 
Figure 59: Object oriented finite element analysis Von Mises residual stress in GPa.

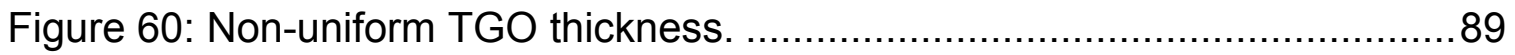

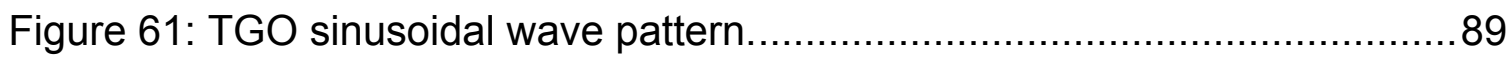

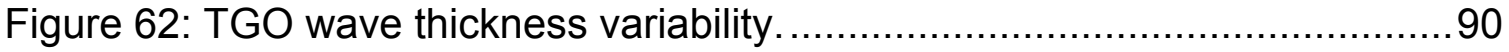

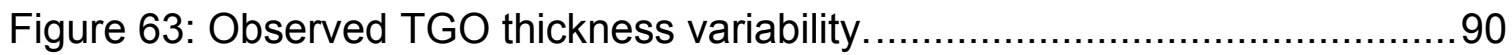

Figure 64: YSZ/TGO/BC interface failure pattern........................................ 91

Figure 65: Thermally grown oxide modeling parameters. ..............................92

Figure 66: Boundary conditions applied to the numerical TBC model................94

Figure 67: YSZ/YGO/BC interface mesh resolution.......................................94

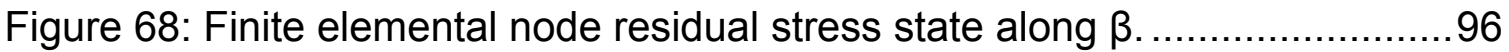

Figure 69: Oxide scale shear stress profile (MPa) ...................................... 96

Figure 70: Oxide scale failure inducing stress pattern created as $\beta$ approaches a

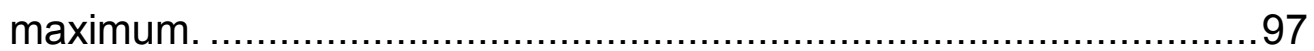

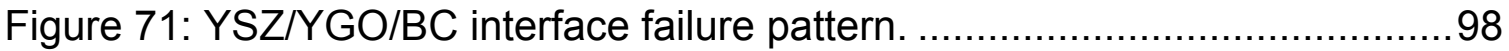

Figure 72: Edge and surface photographs of APS/MCrAIY/RenéN5 coupon A after a cumulative 500 isothermal heat treatment hours at $1100^{\circ} \mathrm{C}$ in an ambient air environment after 2 weeks storage........................120

Figure 73: Surface stiffness response color maps for Coupon A obtained after each isothermal heat treatment.

Figure 74: Edge and surface photographs of APS/MCrAlY/RenéN5 Coupon B after a cumulative 400 isothermal heat treatment hours at $1100^{\circ} \mathrm{C}$ in an ambient air environment.

Figure 75: Surface stiffness response color maps for Coupon B obtained after periodical 40 hour isothermal heat treatment cycles at $1100^{\circ} \mathrm{C}$ in an ambient air environment.

Figure 76: Edge and surface photographs of APS/MCrAlY/RenéN5 Coupon C after a cumulative 400 isothermal heat treatment hours at $1100^{\circ} \mathrm{C}$ in an ambient air environment and 1.5 weeks storage. 
Figure 77: Surface stiffness response color maps for Coupon $C$ obtained after periodical 40 hour isothermal heat treatment cycles at $1100^{\circ} \mathrm{C}$ in an ambient air environment.

Figure 78: Edge and surface photographs of APS/MCrAIY/RenéN5 Coupon D after cumulative 400 isothermal heat treatment hours at $1100^{\circ} \mathrm{C}$ in an ambient air environment and 1.5 weeks storage.

Figure 79: Surface stiffness response color maps for Coupon D obtained after periodical 40 hour isothermal heat treatment cycles at $1100^{\circ} \mathrm{C}$ in an ambient air environment.

Figure 80: Edge and surface photographs of APS/MCrAIY/RenéN5 Coupon E after cumulative 400 cycles of heat treatment at $1100^{\circ} \mathrm{C}$ in an ambient air environment after 2 days in storage.

Figure 81: Surface stiffness response color maps for Coupon $E$ obtained after periodical 40 cycle heat treatments at $1100^{\circ} \mathrm{C}$ in an ambient air environment.

Figure 82: YSZ surface and failure surface photographs of APS/MCrAIY/RenéN5 Coupon $\mathrm{F}$ after cumulative 440 cycles of heat treatment at $1100^{\circ} \mathrm{C}$ in an ambient air environment.

Figure 83: Surface stiffness response color maps for Coupon $\mathrm{F}$ obtained after periodical 40 cycle heat treatments at $1100^{\circ} \mathrm{C}$ in an ambient air environment.

Figure 84: Edge and surface photographs of APS/MCrAlY/RenéN5 Coupon G after cumulative 440 cycles of heat treatment at $1100^{\circ} \mathrm{C}$ in an ambient air environment. 126

Figure 85: Surface stiffness response color maps for Coupon G obtained after periodical 40 cycle heat treatments at $1100^{\circ} \mathrm{C}$ in an ambient air environment.

Figure 86: Surface photographs of EB-PVD/MDL/RenéN5 Coupon $\mathrm{H}$ after a cumulative 640 cycles of heat treatment at $1100^{\circ} \mathrm{C}$ in an ambient air environment. 
Figure 87: Surface stiffness response color maps for Coupon $\mathrm{H}$ obtained after periodical 40 cycle heat treatments at $1100^{\circ} \mathrm{C}$ in an ambient air

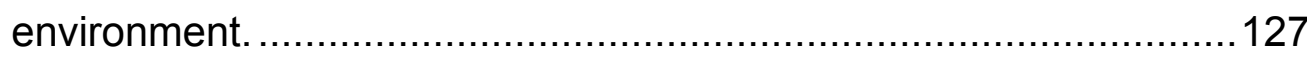




\section{List of Tables}

Table 1: Room temperature elastic modulus measurement............................28

Table 2: Indentation inference zone TBC system material properties [104] ........69

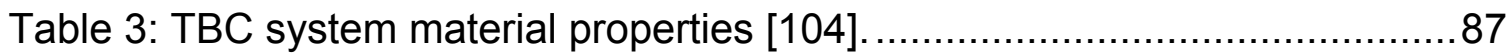

Table 4: Numerical TGO model design parameters......................................93 


\section{Chapter 1: Introduction}

The demand for improved efficiencies in energy conversions systems in recent years has led to the introduction of land-based gas turbines with significantly higher gas inlet temperatures. Handling of such high temperatures requires the use of high strength, directionally solidified and single crystalline nickel base superalloys. In addition, the components of the hottest sections are internally cooled and protected by ceramic thermal barrier coatings (TBC) which allow component operation at gas temperatures in the range of the melting point of superalloys. The efficiency of all types of gas turbine engines, aircraft, landbased and marine, is proportional to the firing or inlet temperature. Increases in these temperatures are facilitated by improved structural design and airfoil cooling technology applied to higher strength cast by increasingly complex processes and coated with steadily improved thermal protection systems. The most critical first stage turbine blades are composed of a nickel-base superalloys in various wrought and cast forms, in addition to being augmented by thermal barrier coatings for increased firing temperatures [1]. Yttria-stabilized zirconia thermal barrier coatings were first suggested as potential utility for land based gas turbines in 1978 [2]. At that time YSZ thermal barrier coatings were in the early stages of development for clean-fueled aircraft gas turbine service. Currently yttria-stabilized zirconia thermal barrier coatings are commonly applied on structural components by an air plasma spray (APS) or electron beamphysical vapor deposition (EB-PVD) process. A metallic bond coat $(B C)$ between the zirconia based TBC and the nickel-base super alloy provides oxidation resistance for the base material and is responsible for adherence of the ceramic coating [3].The yttria-stabilized zirconia primary function is to prevent heat transfer to the metallic substrate. Yttria-stabilized zirconia has reached a level of prominence as a thermal barrier coating due to its relatively high thermal expansion coefficient and low thermal conductivity. These unique properties enable YSZ to thermally protect the structural components of the gas turbine and accommodate the large amount of residual strains that occur upon cooling. 
Forming first during processing and later in service, a thermally grown oxide layer develops as a result of bond coat oxidation [4]. Currently, engine designers still primarily rely on thermal barrier coatings as a hot section component lifetime extension system. However, with the implementation of a reliable spallation failure prediction instrument, researchers may fully exploit the full potential of thermal barrier coatings for significant gas turbine performance improvements.

In today's engines gas temperatures exceed the melting point of superalloys substrates by more than $250^{\circ} \mathrm{C}$ [5]. This is accomplished by the uses of thermal barrier coatings, as well as excessive internal and external cooling, which however reduces overall turbine efficiency. Nevertheless, further increases in thrust-to-weight ratios of the next generation turbine engines will require even higher gas temperatures. To meet these goals, a new production and a complete understanding of current high temperature materials is needed. Additionally, reliable diagnostic methods are needed to evaluate the severity of erosion of TBC systems on land-based turbine engine components.

\subsection{Problem Statement}

One driving force in the development of gas turbines is increased efficiency resulting in less fuel consumption and environmental pollution such as $\mathrm{CO}_{2}$ and $\mathrm{NO}_{\mathrm{X}}$ in the exhaust gases. TBC systems however, coated on hot section components (combustion chamber tiles and rotating blades) of landbased gas turbines increases structural potentials in the resistance against hot corrosive and oxidative environments. TBCs provide performance, efficiency, and durability benefits by reducing turbine cooling air requirements and lowering metal temperatures [6]. These increases in temperature are enabled by the low thermal conductivity of the zirconia YSZ, coupled with active cooling of the underlying metal substrate. A multilayered structure, the TBC is composed of a structural supporting substrate, followed by an oxidation diffusion barrier bond coat on top of which the YSZ is applied. Although the life of these coatings is affected by a number of factors, nearly all researches consider the thermally grown oxide to be a crucial parameter for TBC life [7]. This oxide scale develops 
between the oxygen transparent YSZ and the aluminum rich bond coat. In thermal cycling operation these systems often fail by crack initiation and propagation close to or within the YSZ/BC interface [8]. This failure is attributed to stresses arising from the formation of this thermally grown oxide (TGO) on the rough $B C$ surface. However, the stresses acting in this region are rather complex due to TGO formation, creep effects and roughness of the BC. Furthermore, microstructural interface geometrical conditions between these layers reduce the probability of characterizing residuals stresses that develop in this region. As a result, no accurate numerical failure prediction models have been developed. TBC life cycle estimation techniques lack the accuracy needed for true failure prediction. This is due to both the variability within the system and lack of accurate material property data along system degradation. Furthermore, prevailing non-destructive evaluation techniques although helpful, are only capable of detecting, but not predicting failure.

\subsection{Research Objectives}

Research objectives are summarized and simplified in the following; however a more in-depth description is found bellow:

1. Apply a micro-indentation technique for NDE testing of TBC system materials, where unloading surface stiffness responses of APS and EB-PVD systems subjected to isothermal and cyclic loadings at $1100^{\circ} \mathrm{C}$ will be evaluated.

2. Thermally loaded TBC system cross-sectional scanning electron micrographs will be used to generate numerical models from which residual stress states will be evaluated. Analysis of the out-of-plane tensile stresses developing upon application of a temperature differential will provide a detailed explanation for experimentally observed microstructural failure patterns in TBC systems.

3. Following a classical Hertzian contact mechanics approach, a loadbased multiple partial unloading micro-indentation technique which does not require system calibration or high precision depth sensors 
will be used to development a high temperature micro-indentation system.

With the evolution of nano-technologies, indentation testing techniques have become more prevalent. Yet their ability to evaluate material mechanical properties in harsh environments remains a challenge. Following a classical Hertzian contact mechanics approach, a load-based multiple partial unloading micro-indentation technique has been developed that does not require system calibration or the use of high precision depth sensors, where typical indentation instrument registers the depth of indenter penetration and also any displacement of the instrument arising from reaction forces. These displacements are directly proportional to load and must be accounted for when analyzing the loaddisplacement curve. However with application of a multiple partial unlading algorithm, these constraints have been removed and have led to the development of a portable indentation system and have presented the potential to be applied to high temperature environments as well. In this research, a loadbased multiple partial unloading micro-indentation material mechanical property evaluation algorithms will be enlisted to conducted elevated temperature evaluations of TBC system materials.

Additionally, the durability and life cycle of TBC systems applied to gas turbine blades and combustor components is limiting the maximum temperature and subsequent efficiency at which gas turbine engines operate. The development of new materials, coating technologies and evaluation techniques is required if enhanced efficiency is to be achieved. Of the current ceramic coating materials used in gas turbine engines, YSZ is most prevalent, its low thermal conductivity, high thermal expansion coefficient and outstanding mechanical strength make it ideal for use in TBC systems. However, residual stresses caused by coefficients of thermal expansion mismatches and unstable thermally grown oxides are considered the primary causes for its premature and erratic spallation failure. Sensitive to residual stress state, indentation unloading surface stiffness responses of air plasma sprayed and electron beam physical vapor deposited YSZ TBC systems subjected to isothermal and cyclic loadings at 
$1100^{\circ} \mathrm{C}$ will be evaluated to determine the reliability of micro-indentation as a non-destructive spallation detection system. Furthermore, throughout this study TBC system cross-sectional scanning electron micrographs along ever increasing thermal cycles will be utilized to generate numerical models from which residual stress states will be evaluated. Analysis of the out-of-plane tensile stresses developing upon application of a temperature differential will provide a detailed explanation for experimentally observed microstructural failure patterns in TBC systems. 


\section{Chapter 2: Instrumented Indentation}

Indentation is a mechanical testing process by which material mechanical properties are found. Implementing the use of relatively hard, geometrically specific materials, an indenter is submerged into a test material using a given load for a predetermined amount of time. The material's reaction to this applied load is dependent upon its mechanical properties. The remaining size and depth of the indentation left provides information from which its mechanical material properties are derived. This residual impression's depth and area have been measured using various techniques, including both depth sensing and a range of optical technologies. Initially developed to evaluate only alloys, indentation testing has quickly become helpful to all industrial sectors, including lumber, ceramics, and glass production. Indentation testing's ability to perform nondestructive evaluation on small volumes of material has also made it quite popular in the ever increasing nanotechnology industry. Yet, indentation testing has long been used for hardness testing dating back to the 1800's, but recently these techniques have gone far beyond the capabilities of these original testing procedures and techniques. The demand for nanotechnology has led to the production of much smaller and more precise non-destructive material evaluation procedure. As these materials are develop, indentation methods have moved to the forefront of commonly used evaluation techniques.

First developed to determine mechanical properties of materials that, due to their geometry or configuration, could not be evaluated using conventional methods. Due to this, indentation became the primary technique used for the examination of thin films and coating materials. The mechanics of instrumented indentation are similar to that of a hardness tests where in a rigid indenter is purposely forced into the surface of an unknown sample using a specific load. Yet this is where the comparison stops, although similar, instrumented indentation differs from hardness testing in that indentation size and depth are monitored throughout the evaluation procedure using high resolution displacement or optical sensors. The load-indentation depth plots produced by 
these various methods are ultimately responsible for providing the information required to obtain unknown material mechanical property information. The following is a brief review of these techniques and general contact mechanical theories.

\subsection{Spherical Indentation}

While studying the phenomenon of Newton's ring as a post doctorate, it occurred to Hertz that while much was known about the optical aspect of two lenses being placed together, no good understanding of the deflection occurring at the point of contact had been established [9]. Hertz became particularly concerned by the nature in which localized deformation and pressure between the two spherical surfaces could be quantified, Figure 1 [10].

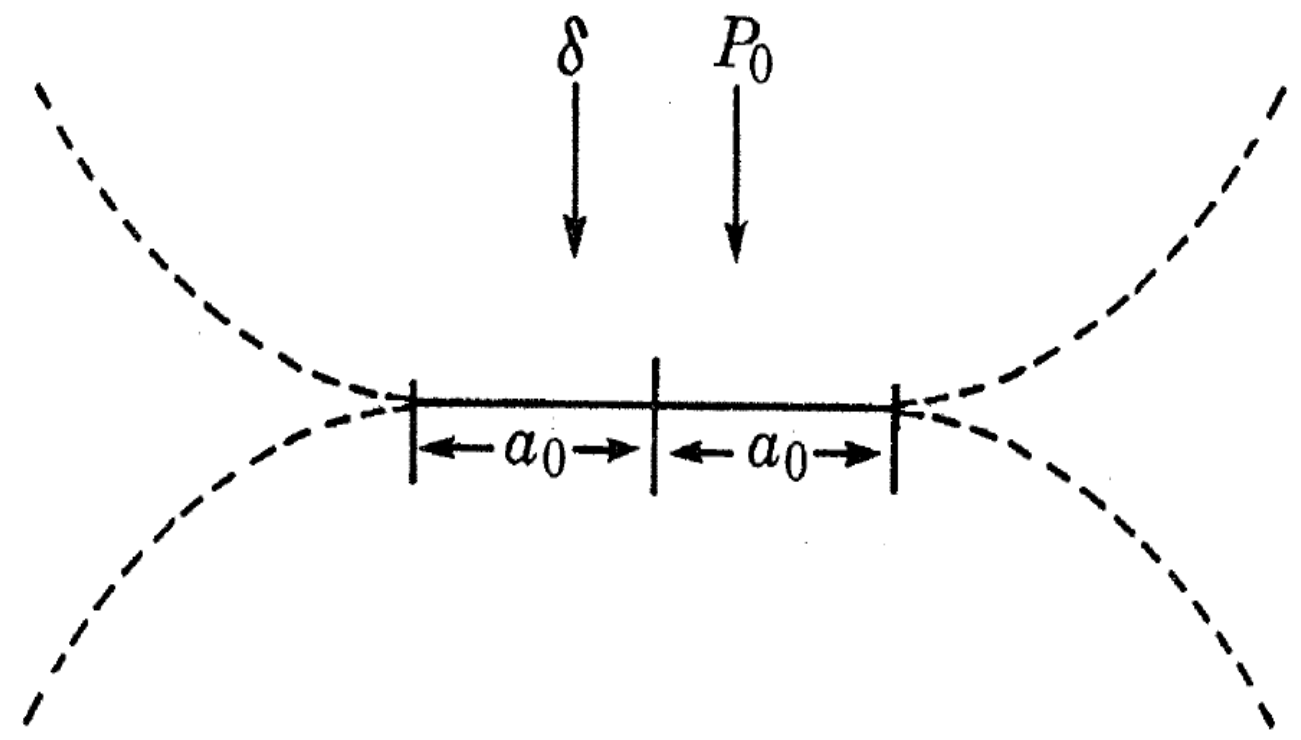

Figure 1: Contact deformation between two spheres [10].

With this in mind, he sought to assign a quadratic function that would represent this profile. What he developed became basis on which all modern day contact mechanical theories are derived, Equations (1) and (2), where $P$ is applied load, $d_{i}$ the diameter of both spheres and $E_{R}$ the reduced modulus.

$$
a^{3}=\sqrt{\frac{3 P}{8 E_{R}\left(\frac{1}{d_{1}}+\frac{1}{d_{2}}\right)}}
$$




$$
\frac{1}{E_{R}}=\frac{\left(1-v^{2}\right)}{E}+\frac{\left(1-v_{i}^{2}\right)}{E_{i}}
$$

Hertz's established that the stresses and deflections arising from the contact between two elastic solids, regardless the shape were subject to geometrical deformation according to this function [11]. Furthermore, allowing the diameter of the second sphere to approach infinity, a load and radius of contact function between a rigid sphere and a flat surface is developed, Equation (3). From this function, Lure developed the following formula for spherical indentation under idealized conditions and the assumption that upon shallow spherical indentation depths $h=a^{2} / R$, Equation (4) where $h_{e}$ represent elastic indentation depth only [12].

$$
\begin{gathered}
a^{3}=\frac{3}{4} \frac{P R}{E_{R}} \\
P=(4 / 3) E_{R} R^{1 / 2} h_{e}^{3 / 2}
\end{gathered}
$$

Throughout spherical indentation of an elastic material, the specimen's surface is typically drawn in and downwards toward the indenter, as a result, a sink in effect around the indenter is observed, Figure 2.

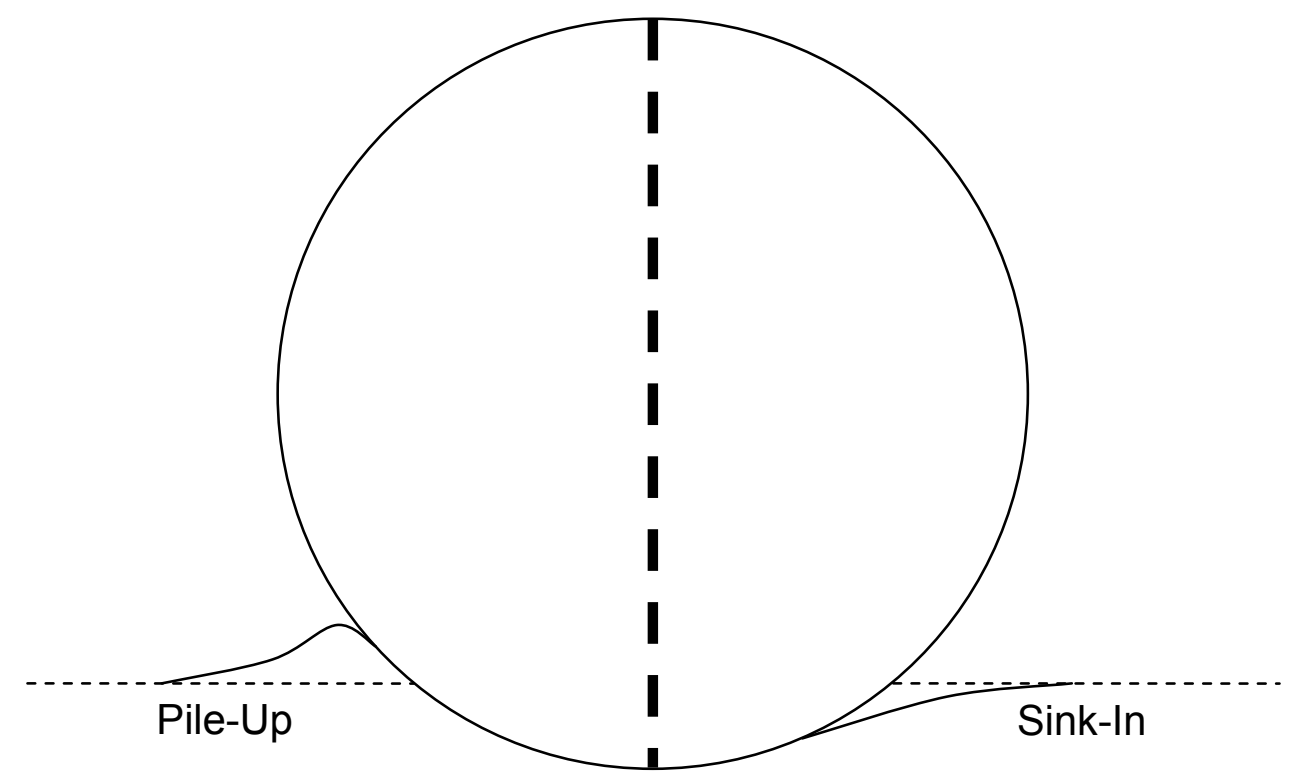

Figure 2: Pile-up and sink-in (not simultaneously) occurring during spherical indentation.

Yet, when the contact involves plastic deformation, the material may either sinkin or pile-up around the indenter. In a fully plastic region, the behavior is found to 
be dependent upon the strain hardening properties of the material and the ratio between elastic modulus to yield stress. For non-strain hardening materials with large $\sigma_{Y} / E$ ratios, the plastic zone is observed to have a hemispherical shape, meeting the surface well outside the radius of the circle of contact. Piling up in these materials is to be expected, as most of the plastic deformation occurs near the indenter. On the other hand, for materials with low $\sigma_{Y} / E$ ratios, the plastic zone is contained within the circle of contact boundary. The elastic deformations that accommodate this volume of indentation are then spread to a greater distance, resulting in a sinking in effect [13]. For materials that exhibit strain hardening, the yield strength effectively increases as the strain increases. For this reason, during an indentation test, the material within the plastic zone becomes more rigid as the amount of deformation increases. Due to this, the material close to the indentation solidifies, yet the surrounding material remains elastic. As a result, the plastic region is driven deeper into the specimen and the material encircling the indentation is then subjected to a sinking in effect.

\subsection{Instrumented Indentation Data Analysis}

Elastic modulus and hardness values among others are typically parameters obtained from instrumented indentation tests. Yet, unlike a typical hardness test, wherein indentation impression size is measured directly, instrumented indentation rather calculates this value with knowledge of the indenter's geometry and indentation depth. Due to this, instrumented indentation is often referred to as depth sensing indentation testing as well. Although instrumented indentation may be performed using indenters of any shape, sharp and spherical geometries have become more popular in recent years. Ultimately resulting in the extraction of elastic modulus and hardness information from the test material, load and depth curves are recorded as load is applied to the indenter and consequently the specimen as well. This applied load creates

plastic deformation within the specimen directly beneath the indenter tip, causing a residual impression to remain following removal of the load, yet unlike conventional hardness test, the size of this impression is far too small for 
conventional optical measurement techniques. In response to this, the depth of penetration together with the known indenter geometry provides an indirect measurement of contact area, from which an estimate of hardness is made. Elastic modulus calculation however, requires the acquisitions of additional parameters, the theory and details of which are discussed in the following sections.

\subsubsection{Single Point Unloading}

Single point unloading indentation tests use, as the name implies, only one unloading slope within the load-displacement curve from which the reduced modulus is calculated, Figure 3.

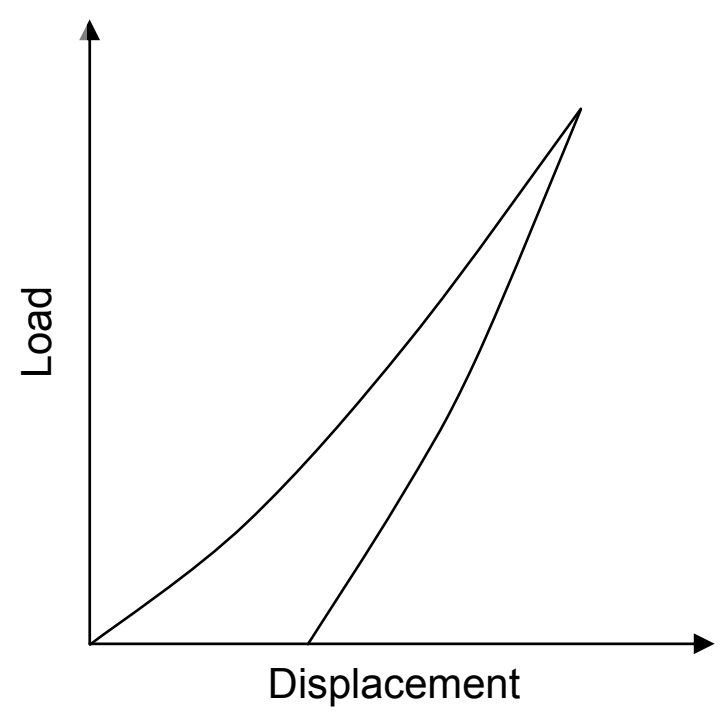

Figure 3: Load displacement curve for single point unloading algorithm.

This method, employed by the majority of instrumented indentation systems provides an accurate and theoretically sound measurement of the materials elastic response. Through an extraction of the load-displacement curve's loading slope, elastic modulus is calculated via Equation (5) where contact area is calculated using a known indenter geometry and the indentation depth $\left(A=\pi a^{2}\right)$ [14].

$$
E_{R}=\frac{1}{2} \frac{d P}{d h} \sqrt{\frac{\pi}{A}}
$$


Although this testing technique has many advantages, its greatest attribute may be the shallow penetration depths required. Quite popular in the material research community, shallow penetration depths has allowed indentation testing techniques to be deemed relatively non-destructive. Furthermore, these superficial penetration depths required permit samples composed of multiple layers, such as thin films, to be evaluated without interference from the substrate's material properties. Additionally, the simplicity and effectiveness of this technique has enhanced its marketability and is used in many commercially available instrumented indentation systems.

\subsubsection{Multiple Partial Unloading}

Developed at West Virginia University, the first generation of instrumented indentation analysis data reduction techniques required the use of transparent sapphire spherical indenter. This transparent indenter measurement (TIM) system, with phase shifting Twyman-Green and Moiré interferometer techniques directly measures the out-of-plane deformation and contact radius throughout the indentation process [15]. The variation in out-of-plane deformation and continuously measured contact radii provide a platform on which the material's elastic modulus and true stress strain relationship are found. This technique although easily performed in a lab environment, requires the use of sophisticated optical systems. Through this direct contact area measurement along with the unloading slope, the material's elastic modulus is calculated via Equation (5). Although unique and novel, this technique's versatility is limited due to its optical requirement. As a result of this, a second generation TIM data reduction technique was developed wherein a partial unloading produce was employed, Figure 4. 


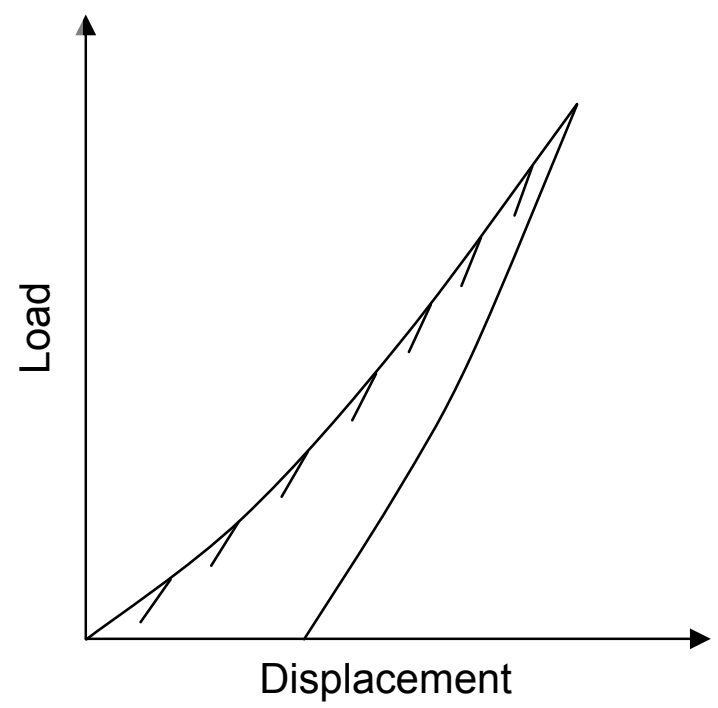

Figure 4: Load displacement curve for multiple partial unloading algorithm.

This simplified transparent indenter measurement method however does not require the interferometric optics used in the first generation. Furthermore, this second generation TIM system was able to continuously monitor contact area by an exchangeable long working distance microscope [16]. Through this, contact area measurements were captured at each unloading.

The basic principle on which this data reduction techniques operates is the assumption that throughout an indentation test, total displacement of the actuator is not only a function of the indentation depth but compliance of the system as well. As a result, total displacement $\left(h_{\text {total }}\right)$ is the summation of both total indentation depth $(h)$ as well as system deflection $\left(h_{s}\right)$, Equation (6). Furthermore, as the indentation unloading response is solely elastic, it can be assumed that elastic compliance $\left(d h_{e} / d P\right)$ is equal to total indentation compliance $(d h / d P)$. Thus taking the derivative of this function with respect to indentation load $(P)$ and replacing elastic compliance with indentation compliance leads to the development of Equation (7).

$$
\begin{gathered}
h_{\text {total }}=h+h_{S} \\
\frac{d h_{\text {total }}}{d P}=\frac{1}{2 E_{R}} \sqrt{\frac{\pi}{A}}+\frac{d h_{S}}{d P}
\end{gathered}
$$


However, with this equation the reduced modulus determination still requires an in-situ contact area measurement, yet only total displacement rather than indentation depth is needed. The removal of this not easily acquired parameter alleviates much of the error associated with load-depth sensing indentation. Furthermore, under the assumption that system compliance $\left(d h_{S} / d P\right)$ remains constant throughout a given indentation test, a function containing only differences in contact area and slope between partial unloadings is developed, Equation (8). From this it is seen that only contact area and unloading slope are non-constant variables, thus a function needing only contact area and total displacement is developed for the extraction of reduced modulus.

$$
E_{R}=\frac{1}{2} \sqrt{\pi}\left(\left.\frac{1}{\sqrt{A}}\right|_{2}-\left.\frac{1}{\sqrt{A}}\right|_{1}\right)\left(\left.\frac{d h_{\text {total }}}{d P}\right|_{2}-\left.\frac{d h_{\text {total }}}{d P}\right|_{1}\right)^{-1}
$$

This simplified transparent indenter measurement method however removing the interferometric optics needed in generation one still requires the use of optical contact area measurement. Furthermore, although this technique and function are proven, the evolution of a load based equation would alleviate much of the cost associated with acquisition of elastic modulus by instrumented indentation techniques. Thus, the third generation TIM employs a variation of the multiple partial unloading technique developed for the second generation of instrumented indentation analysis. Yet, unlike the second generation, this analysis procedure does not require any optical contact area measurement. Thus, rewriting Equation (4) in the form $P=\alpha h_{e}{ }^{m}$, solving for $h_{e}$ and taking the derivative of both sides with respect to $P$ yields Equation (9), where $\alpha=(4 / 3) E_{R} R^{-1 / 2}, m=3 / 2$ and $C=(1 / m) \alpha^{-1 / m}$.

$$
\frac{d h_{\text {total }}}{d P}=C P^{(1 / m)-1}+\frac{d h_{S}}{d P}
$$

It is shown via Equation 2.9 that total displacement compliance $\left(d h_{\text {total }} / d P\right)$ and $P^{-1 / 3}$ maintain a linear relationship, if and only if system compliance $\left(C_{S}\right)$ remains constant throughout the multiple partial unloading steps. It is from this that the slope $(C)$ can now be used in the determination of the reduced modulus, Equation (10). 


$$
E_{R}=\left(6 m^{3} R\right)^{-1 / 2}
$$

Previous indentation methods using only single point unloading are incapable of obtaining this slope, thus require contact area or indentation depth measurements. Also, conventional indentation methods develop error in elastic modulus measurement along applied load. Specifically, if a large load is applied the resulting elastic modulus will too be large. Moreover, this scenario holds true for small applied loads as well, where in a relatively diminutive applied load results in an underestimation of a materials true elastic modulus. The multiplepartial unloading method on the other hand eliminates this loading influence. The validity of the proposed load-based indentation method can be addressed via Equation (10), where if system compliance $\left(C_{S}\right)$ remains contact throughout the indentation test, this linear relationship will be maintained.

\subsection{Instrumented Indentation Systems}

Despite the simplistic design and setup, the indentation process requires considerable amounts of experimental experience and resources. The high precision of these instruments demand a familiarity of load-depth sensing indentation testing. Due to the extreme sensitivity of indentation chain components, it is necessary that the specimen and instrument be free from vibration or tampering of any kind. Implementing the use of an optical table will ensure a steady test platform but is not completely necessary. Furthermore, the instrument should not be subjected to drastic temperature changes, as this will prevent any errors due to thermal expansion effects. These and other considerations should be taken into account prior to the construction of any indentation system. Additionally, sample and loading frame geometry should be considered throughout the design process as well. Moreover, all indentation systems require the use of a rigid loading frame. This component of the indentation system is responsible for creating the boundary conditions under which the equations for ideal contact mechanics are derived, unfortunately some level of system deflection exist. This system compliance is unavoidable, yet greater accuracy is achieved when this value is minimized. The following 
presents the components of the indentation chain in greater detail and those systems developed for this research. Encompassing some capacity of a geometry specific loading frame, load cell, piezoelectric actuator and indenter, Figure 5, the components and structures of these units are discussed in the following sections. Finally, all indentation chains components must meet the standard practices for instrumented indentation testing developed by ASTM International [17].

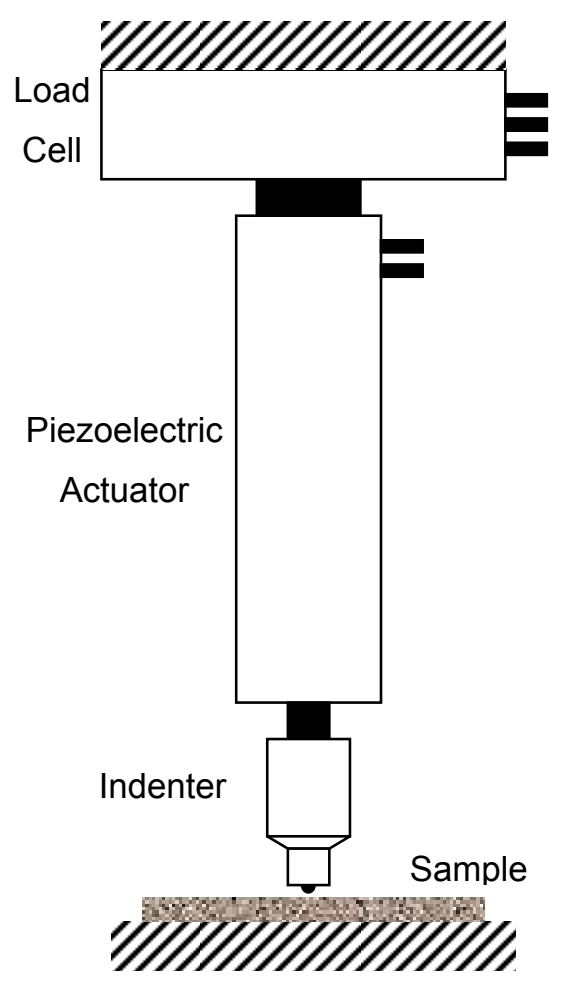

Figure 5: Instrumented indentation system loading chain schematic.

\subsubsection{Load Cell}

The load cell or force transducer used in an indentation system is enlisted to convert a load response to an electrical signal. Stress analysis is often performed by measuring strains on the surface of deformed elements. Of the many experimental methods available, the electrical resistance strain gage is the most common choice of the great majority of experimentalist [17]. Used for its size and accuracy, strain gauge load cells are extremely reliable. When applied to a know material with a known geometry, a respective load can be determined. 
It is by this principle that a strain gauge load cell operates. However, the voltage drop across these elements is rather subtle and requires the services of a Wheatstone bridge configuration. Allowing these minute variations in voltage to be measured, the Wheatstone bridge acts as amplification source for electrical load signals to be acquired throughout the indentation test [19].

\subsubsection{Actuator}

Due to its high resolution, a piezoelectric actuator is typically used the displacement source for depth sensing indentation applications. Composed of multiple piezoelectric ceramic discs, the actuators displacement is primarily a function of the applied electric field. As a voltage is applied to these materials, mechanical strain (displacement) is produced similar to that of thermal expansion. However, as electric fields and not temperature gradients are used, a faster reaction time is achieved resulting in an increase in sensitivity. The active material of the positioning element is composed of piezoelectric disks. Separated by thin metallic electrodes, this stack of elements exhibits a great deal of stiffness at high pressures and is able to provide stability throughout the indentation test [19]. Factors such as thermal drift and overheating need to be considered when using a piezoelectric actuator. Although minute, these factors may cause errors in a system requiring such precision.

\subsubsection{Indenter}

The indenter material and geometry must be chosen based on the information one wishes to obtain from the indentation test. Furthermore, the method by which the indenter is attached to components of the indentation chain must be secure. Sharp indenters, such as Vickers, Berkovich and Knoop, induce plasticity earlier in the indentation process. This is desirable when testing thin films for which the hardness or modulus of the top coat, independent of the substrate, is required. Spherical indenters, in opposition to that of sharp, offer a gradual transitions from elastic to elastic plastic response. This gradual transition enables strain hardening characteristic of test materials to be examined as well. 
While geometry is of great importance, the material of which the indenter is composed is significant as well. This material must quite rigid and strain resistant. Due to this, materials having a relatively high elastic modulus are often used. Common materials include, sapphire, tungsten carbide and diamond [19]. Although brittle, sapphire indenters are highly desirable for their optical properties. For this and cost, sapphire indenters are often used in experimental capacities.

\subsubsection{Loading Frame}

The loading frame of an instrumented indentation device must be of heavy construction. Often composed of steel, the loading frame becomes the most important aspect of an indentation system under loading conditions. The accuracy of results obtained from an indentation system in highly proportional to the level of compliance in the indentations systems loading frame. Due to this, a great deal of time and effort is required for the design and construction of these systems. Furthermore, the loading frame must be able to accommodate specimens of various sizes and geometries. This requirement presents a problem for indentation system designers as the loading frame must also act as a rigid body throughout the indentation test. In order to minimize system compliance, a rigid stabilizing or clamping element must be utilized at each joint [20]. Other issues such as weight and size should also be considered, where a system of great magnitude may perform well but is also confined to lab. Additionally, the more portable the system is the less likely it is to act as a rigid body. Yet, evaluation of immobile specimens such as bridge trusses and building columns are commonly desired. This requires that some balance between rigidity and portable be met, yet accuracy of the evaluation technique remaining most important.

\subsection{Factors Affecting Instrumented Indentation}

Although theoretically simple, in practice a range of errors are associated with experimental instrumented indentation testing. Many of inaccuracies arise from environmental or instrumentation malfunctions throughout the indentation 
process [19]. In addition, there are a number of material related issues that also affect the validity of these results. Moreover, the sensitivity of an instrumented indentation test to these phenomena and others is the subject of much debate and continuing research. Yet, an attempt to subside these errors is futile as many are often unpredictable and unavoidable. Of the many errors that are capable of reducing the reliability of an indentation test, system compliance may be the most important. Large deflection of the loading frame results in shallow indentation depths and ultimately inaccurate material property measurements. Experience shows that the errors caused by this significantly affect the slope of the fitted line at low applied loads. As a result, the rigidity of the indentation system must be proven prior to experimental characterization. Additional parameters limiting the accuracy of load depth sensing material characterization are discussed bellow.

\subsubsection{Residual Stress}

In instrumented indentation analysis procedures it is assumed that the specimen is stress free. Yet, materials often contain immeasurable stress patterns. This level of residual stress within the material may be found by examining the shape of the pile-up occurring at the edge of the indentation. These deviations in shape give some information about the level of residual stress within the specimen. However, the load displacement curve also provides some insight to this matter. Experimental techniques have shown these effects to be too small to measure directly. However, general reports of smaller indentation depths have been observed if a compressive residuals stress exists and larger depths if tensile stresses are present. It was later recognized that for elastic contact, any residual stresses within the specimen serve to alter indentation stress and strain distributions [21]. More recently, researchers have demonstrated that residual stresses within a material are shown to alter the results of indentation based mechanical property measurements as well [22]. Hardness and elastic modulus results using standard nano-indentation techniques and data analysis procedures were found to increase when 
performed on materials under uniform compressive stresses and reduce under uniform tensile stresses, Figure $6[23,24]$.

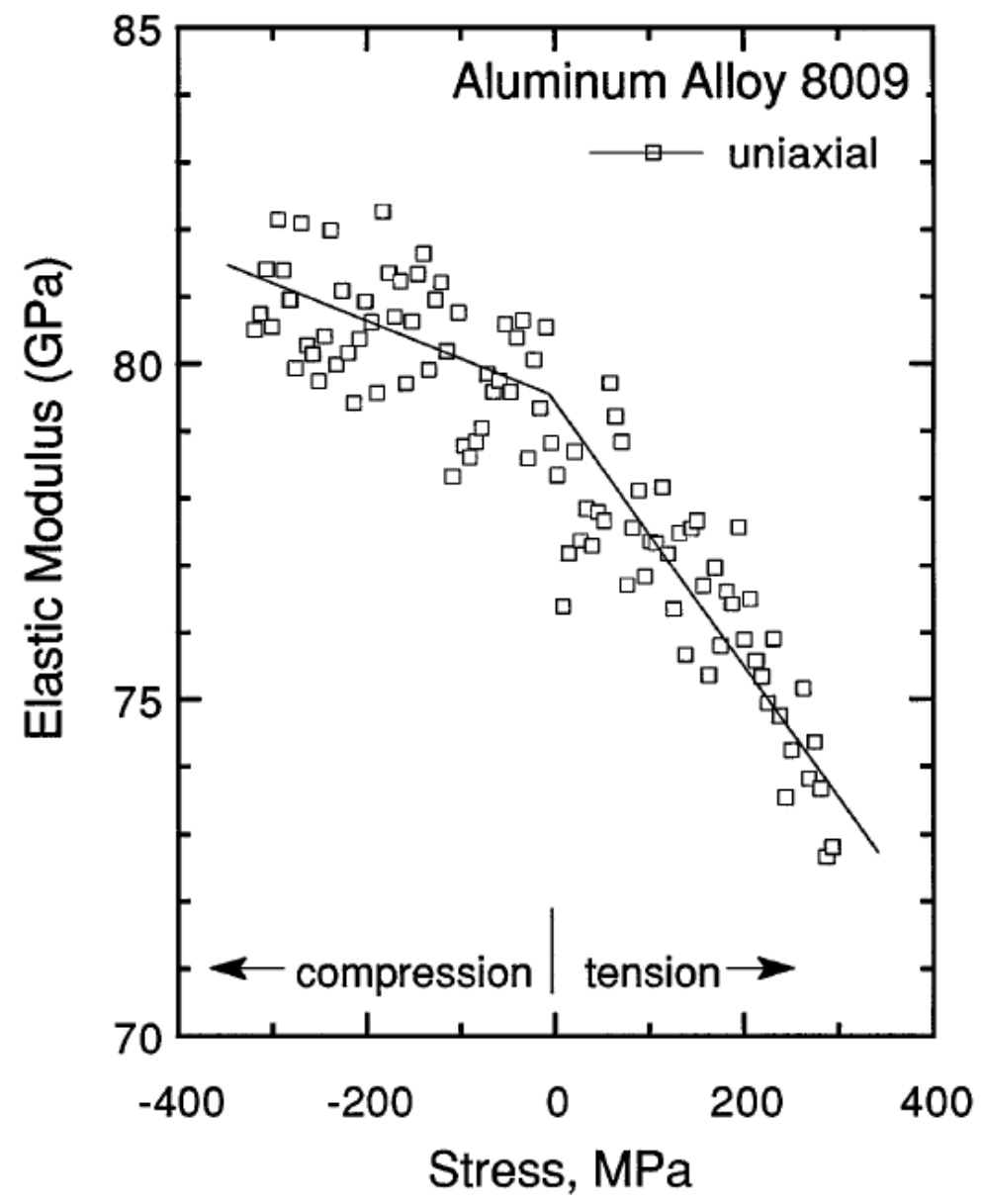

Figure 6: Elastic modulus variance with an imposed residuals stress [23].

Furthermore, finite element simulations have demonstrated the significant effects of biaxial residual stresses on load and displacement parameters of spherical and sharp indentation citing an increase in calculated elastic modulus and unloading slope as well as decreases in total contact area [25, 26, 27, 28]. Additionally, general methodologies have been proposed for the determination of surface residual stresses and residual plastic strains using instrumented indentation techniques [30, 29]. Moreover, throughout analysis of the indentation load required to induce cracks, a numerical method has be developed capable of measuring residual stresses in small volume brittle materials [31]. These models although well investigated are unable to detect the presence of residual stresses 
in an unknown material. Thus require evaluation of both a stressed and unstressed volume to establish states of compression or tension.

\subsubsection{Surface Roughness}

As contact area is measured indirectly from depth of penetration, sample surface roughness is capable of inducing considerable error in the determination of elastic modulus, Figure 7 [32].

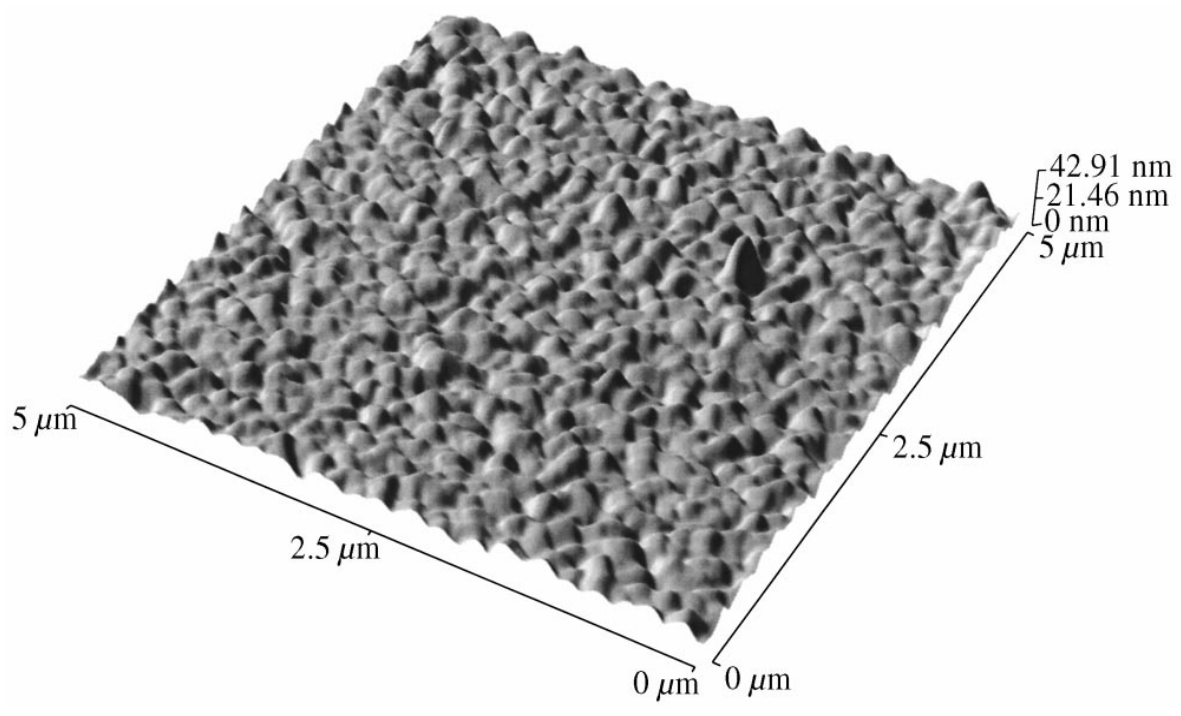

Figure 7: Atomic force micrograph of a $0.76-\mathrm{mm}$ thick aluminum film [32].

Surface roughness is characterized by the asperity height and spatial distribution across the specimen surface, where the parameter is equal to maximum asperity height, indenter radius and contact area radius obtained under a given load. With this parameter, surface roughness is found to affect an instrument indentation test if values greater than 0.05 are observed [33]. The overall effect of surface roughness on the indentation test is the reduction of mean contact pressure, and thereby a simulated increase in calculated contact radius. For a given indentation load and depth of penetration, the calculated values will be far less and result in a reduction in measured elastic modulus. The use of a sharp indenter will alleviate much of this error, yet a high level of surface roughness supersedes the effectiveness of this substitution. 


\subsubsection{Drift}

There are two types of drift behavior that may be observed during instrumented indentation, both creep and thermal drift. Manifesting most clearly as the indentation load is held constant, creep will cause the indentation depth to increase as it is attempting to keep a constant load. This effect, typically unwanted during instrumented indentation testing, can also be desired in some instances as instrumented indentation is also able to perform creep exponent evaluation. This consequently creates yet another material property that may be extracted through indentation testing. Virtually indistinguishable from creep, thermal drift, causing a change in specimen dimension may also be observed during indentation testing both at room and elevated temperatures. Thermal expansion, resulting in a changing of indentation depth, imposes error onto the load and depth readings gathered by the load cell. Yet if total indentation time from start to finish is relatively small, thermal drift has been shown to have a minimal effect on the calculated elastic modulus [34]. Additionally, if the testing temperature is at a steady thermal state, thermal drift effects will not be observed.

\subsubsection{Indentation Size}

For an isotropic homogeneous material one would expect to measure only one elastic modulus value, yet for a variety of reasons, experimental data often results in a variation of this value due to total indentation depth. This effect may be a real reflection of the material's behavior and arise due to the presence of thin oxide films which substantially differ in material mechanical property than that of the substrate [19]. Furthermore, for materials exhibiting indentation size effects, the conditions of plastic flow may depend not only on the strain but also the magnitude of strain gradients present within the material. These gradients may occur in the vicinity of a crack where the stress fields are rapidly changing or even near an edge. Typically defects of this nature will result display significant reductions in surface stiffness response as indentation depth increases, particularly in the event of shallow cracking. 


\subsection{High Temperature Indentation}

Instrumented micro-indentation techniques have generally been developed for room temperature tests; however more recent research activities have resulted in the extension of this technique to elevated temperature and controlled environment material mechanical property characterization. Microindentation hardness tests performed on jadeite and diopside at temperatures between $300^{\circ} \mathrm{C}$ and $750^{\circ} \mathrm{C}$ have been conducted with high levels of accuracy [35]. Additionally, further showing that low thermal drift can be achieved; a high temperature stage for small scale material property characterization to $400^{\circ} \mathrm{C}$ was developed [36]. Later, researchers using a sapphire hemispherical indenter presented a micro-indentation probe for measuring the plastic deformation of porous thermal barrier oxides at temperatures between $937^{\circ} \mathrm{C}$ and $1137^{\circ} \mathrm{C}$, obtaining load and load-line displacement curves of TBC systems, Figure 8 [37].

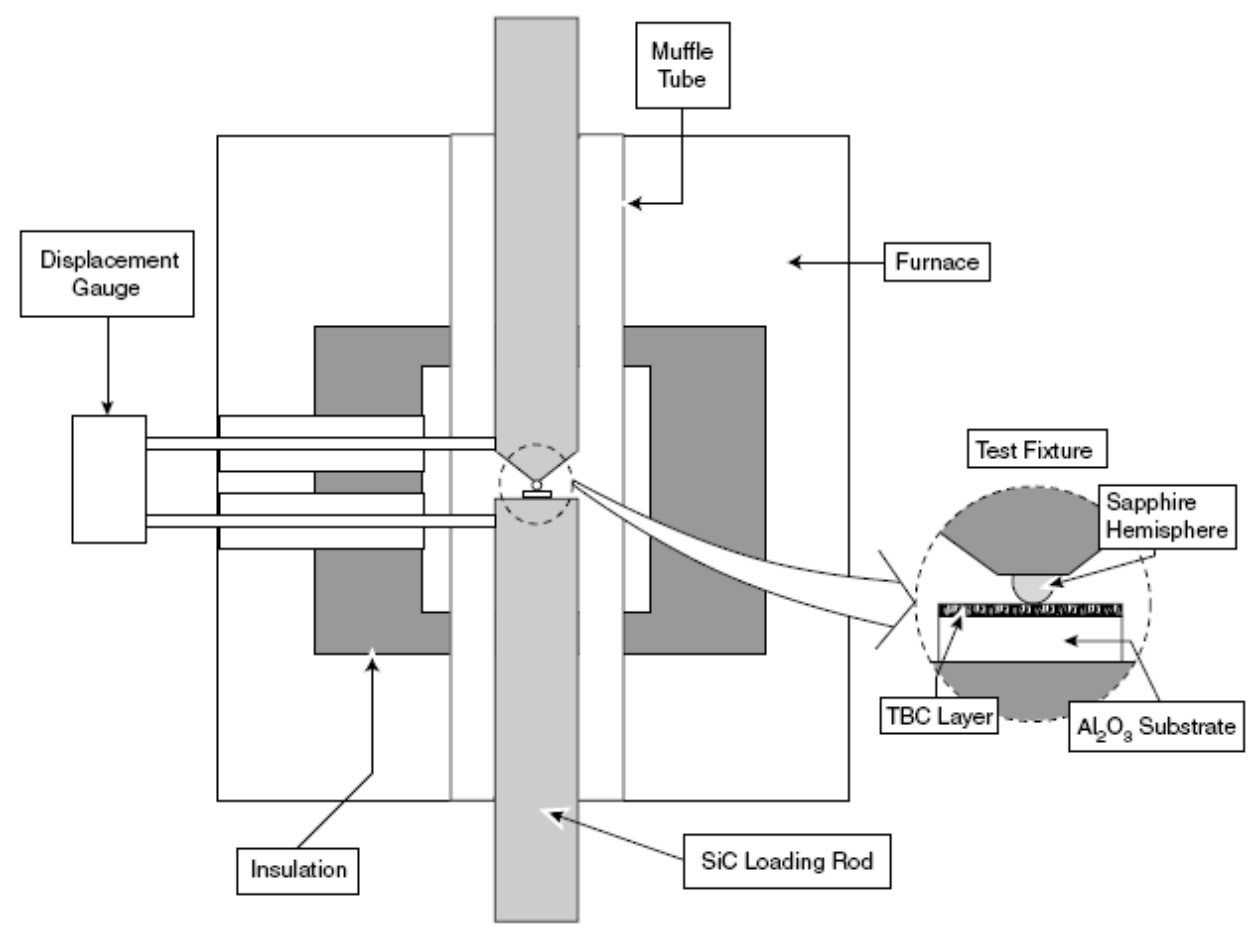

Figure 8: Micro-indentation probe for measuring plastic deformation of porous thermal barrier oxides at temperatures between $937^{\circ} \mathrm{C}$ and $1137^{\circ} \mathrm{C}$ [37].

These high temperature impression tests performed on various thermal barrier oxides deposited by EB-PVD revealed the columnar microstructure 
developed by this coating application procedure caused this material to be susceptible to heterogeneous deformation in the form of shear bands. These bands were found to be accompanied by cracks that introduce a plane of weakness, resulting in susceptibility to material removal during impact by foreign objects. More recently, an original instrumented micro-indentation system capable of testing materials in inert atmosphere environments up to $1000^{\circ} \mathrm{C}$ was developed. The validity of this system was evaluated using $\mathrm{NiAl}(\mathrm{Pt})$ thermal barrier bond coating materials and showed comparable agreement with reported literature values [38].

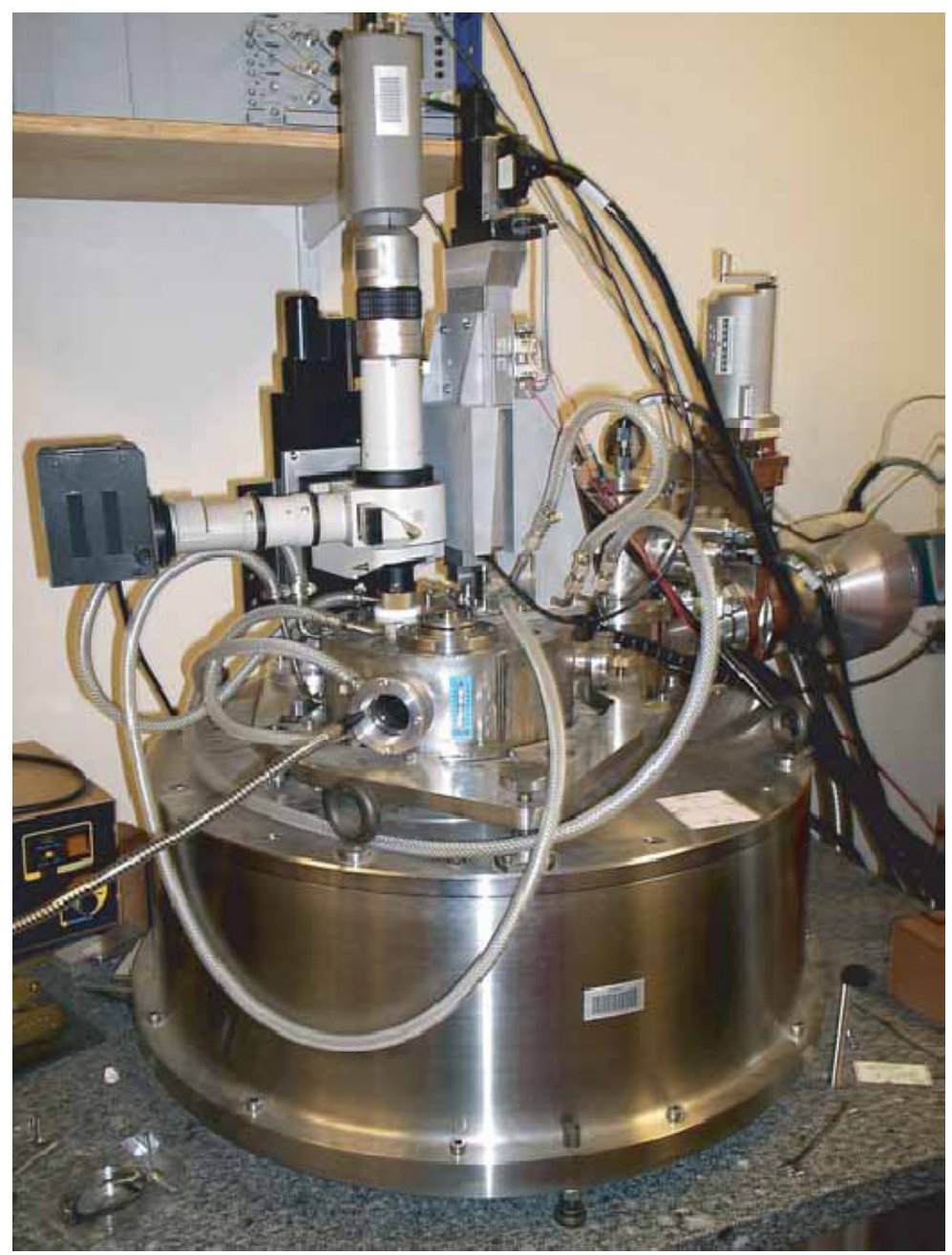

Figure 9: Instrumented micro-indentation equipment for experiments up to $1000^{\circ} \mathrm{C}$ [38].

Later, quantitative mechanical property measurements were performed on a specimen of standard fused silica at temperatures up to $405^{\circ} \mathrm{C}$, ultimately 
showing good correlation with accepted hardness and elastic modulus values [39]. In this research a high temperature indentation system has been developed utilizing the previously detailed multiple partial unloading procedure for material mechanical property evaluation. This load based technique has allowed to the development of an instrumented indentation system capable of reaching temperatures in excess of $1200^{\circ} \mathrm{C}$, the details and results of which are described bellow.

\subsubsection{System Development and Description}

Prior to the development of this high temperature indentation system, validation tests at room temperature were first conducted. To perform these tests, three room temperature only indentation systems were developed, each of which is designed to accommodate samples of various geometries. Verification tests of both a table top and portable load-depth sensing indentation system were performed, the details of which are discussed elsewhere [26]. The results of these experiments shown that this multiple partial unloading procedure is capable of accurately obtaining elastic modulus values of metallic alloys with both flat and tubular surfaces. Furthermore, this technique required only minimal surface preparation, consisting of; a) minor polishing using 600 grit sand papers, followed by b) a brief acetone bath removing any particulate that may have remained. The general design principles of these validation micro-indentation systems were utilized in the development of the high temperature material mechanical property evaluation apparatus, the details of which are presented bellow.

Utilizing the proven designs principle of previously constructed room temperature indentation systems, a simple low compliant loading frame structure was developed [40]. Encompassing guide rails and boundary constraints, this design allows the indentation chain to be both maneuverable yet rigid once secured, Figure 10. 


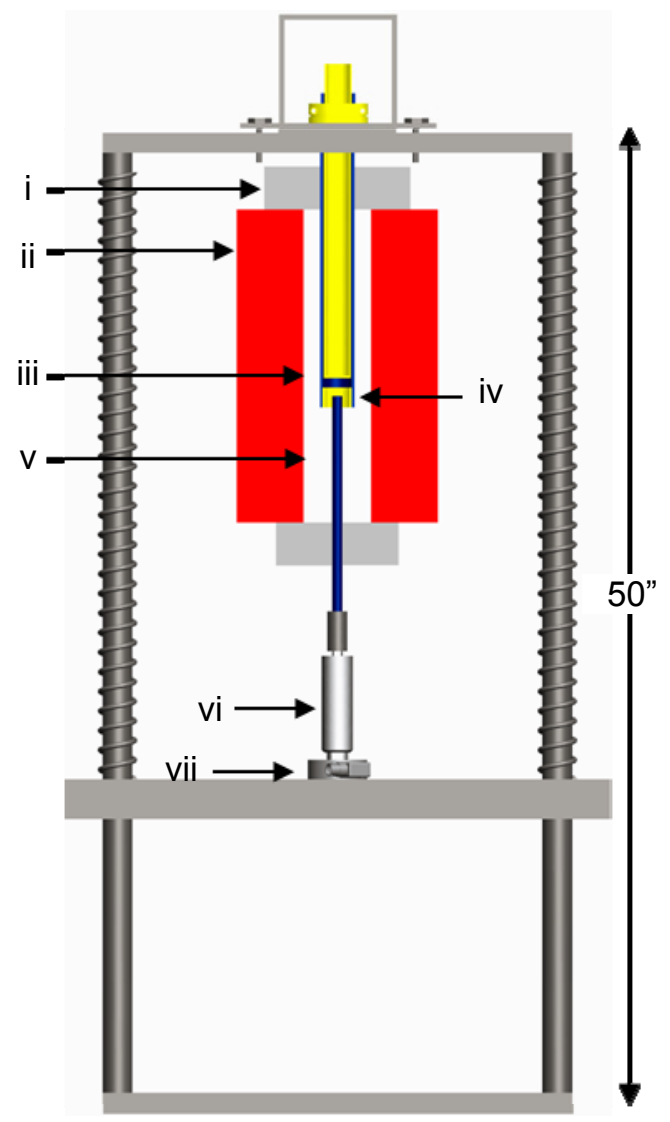

(a)

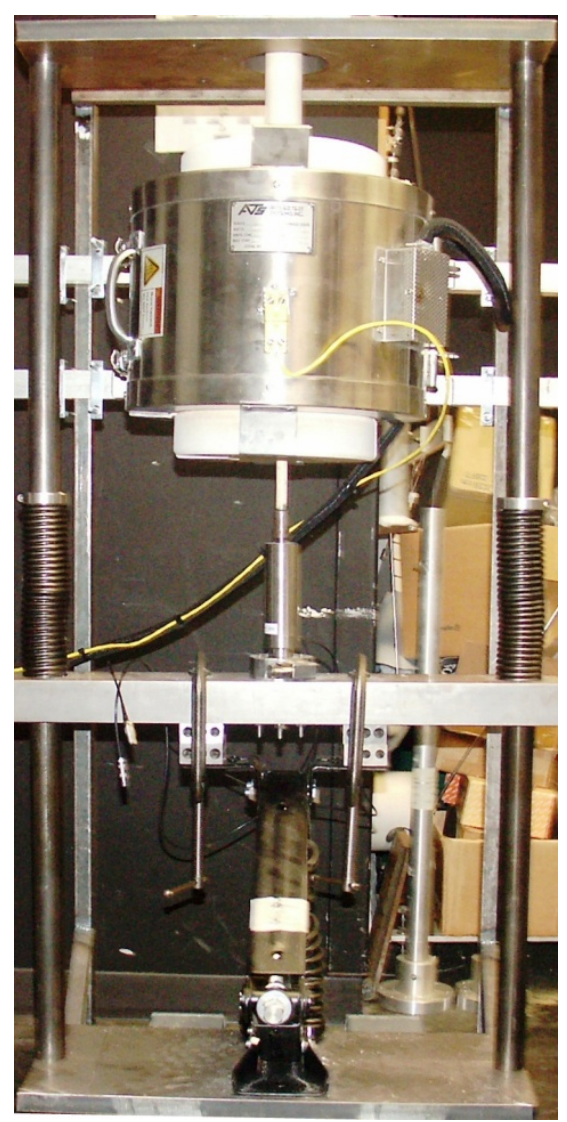

(b)

Figure 10: High temperature indentation system loading frame design; a) i: glass fiber insulation, ii: furnace heating element, iii: sample, iv: spherical sapphire $\mathbf{7 5 0} \mu \mathrm{m}$ radius indenter, v: alumina indenter rod, vi: $0.8 \mathrm{~nm}$ resolution Physik Instrumente P-246.7S 120 $\mu \mathrm{m}$ piezoelectric actuator, vii: $\pm 0.1 \%$ accuracy Honeywell Model $75100 \mathrm{lb}$. load cell and b) optical image.

Due to the relatively high temperatures of which this system will be exposed, a custom indenter assembly was developed wherein a semi-spherical sapphire lens was linked to the center of an alumina rod using ceramic glue. Although the mechanical properties, namely elastic modulus, of sapphire are found to be inversely proportional to temperature, these reductions are minimal when bellow $1400^{\circ} \mathrm{C}[41,42]$. This design ensured the highly delicate and temperature sensitive components of the indentation chain not be exposed to the thermal loads. Additionally, to further reduce the temperature to which the indentation chain was exposed, an inverted indentation system design was 
implemented where the sample and indentation chain positions are reversed. This inverted design allowed all heat lost to radiate in the direction opposite to that of the indentation chain. However, as a result of this inverted design, a unique sample constraint system had to be developed, Figure 11.

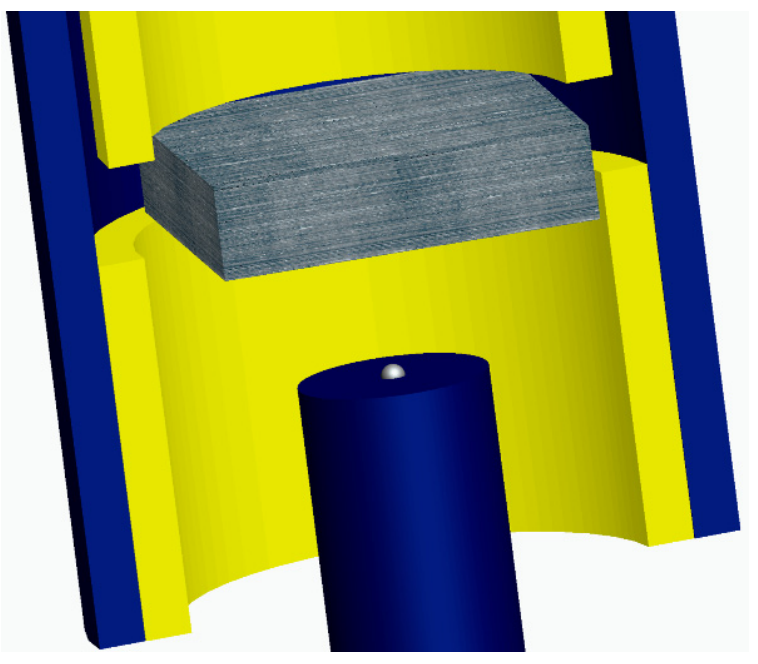

(a)

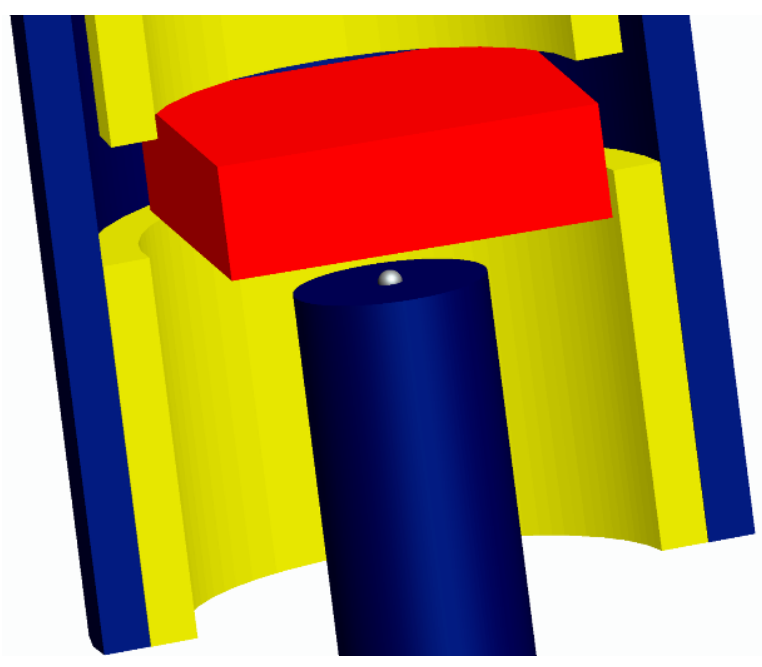

(b)

Figure 11: High temperature indentation system design, a) room temperature and b) high temperature.

Composed of both an outer and inner ceramic tube, this system is responsible for creating the boundary conditions required for idealized indentation throughout testing. Utilizing opposite directional forces, the sample in clamped between the base of the inner and shelf of the outer ceramic tubes. This method allows the specimen to only be constrained once the desired test temperature is reached. Due to variations in thermal expansion mismatch between the sample and the ceramic tubes, the opposite directional forces are not applied until the system has had ample time to reach steady state conditions. Upon reaching this target temperature, the inner ceramic tube is lowered to prevent upward movement of the coupon. As a result of this method by which the samples are constrained, prior to performing a test with this unit, coupons must first be cut to a size of 1 " by 1 ". The thickness of the coupon may vary yet a minimum thickness of $1 / 8$ " in desired, this minimizes any warping of the sample once the boundary constraint is applied. Following sectioning, the sample in then 
polished using standard material preparation techniques with 600 grit sand papers. Any remaining film that may have deposited on the surface is then removed using an acetone solution. Once complete, the sample in placed in the stage with the polished side facing the indenter and positioned in the high temperature evaluation system. The sample remains in this position until the furnace has reached a desired steady state condition. As previously mentioned, the sample may not be constrained prior to high temperature exposure due to variation is thermal expansion coefficient between it and the ceramic sample stage.

Having reached steady state thermal conditions (the time required to reach these conditions increases with set temperature), the sample is now constrained using a simple loading thread and lock design validated prior to construction of this device. Once complete, the sample carriage is constrained via threaded locks as well and will remain in the position until evaluation is complete. As the sample is now secure, the positing of the indentation chain is begun. This process requires that the indenter, load cell and actuator be brought to within reach of the sample's surface. Prior to raising the indentation chain, the actuator is manually driven to a position of relatively small expansion $(\sim 20 \mu \mathrm{m})$. As the indentation chain is slowly raised, the load cell indicator is closely monitored for any variation. Once the indenter has made contact with the sample surface, the actuator is then promptly retracted once again. This pre-expansion and subsequent retraction of the actuator allows any additional expansion of the alumina rod of which the sapphire indenter is attached to subside prior to execution of the indentation test. This process is necessary in that although a majority of the indenter/alumina rod has been exposed to steady state temperature, the additional length of which the indentation chain was raised has not. Following this step the sample is moved to a fresh (non-indented) location and the system is once again allowed time to reach steady state. The system is now ready to perform evaluations and the multiple partial unloading procedure is executed. Although extensive, this pre-evaluation procedure is required if accurate and consistent data is desired. These thermal loads applied throughout 
testing are done so using an Applied Test Systems ${ }^{\mathrm{TM}}$ resistance coil tube furnace capable of reaching a maximum temperature of $1250^{\circ} \mathrm{C}$ with a sensitivity of $\pm 3^{\circ} \mathrm{C}$. Although this temperature was maintained throughout the thermal chamber, the true sample temperature is not monitored.

\subsubsection{Results and Discussion}

Assuming a Poisson's ratio of 0.3 and 0.34 , room temperature elastic moduli for H13 Tool Steel and Bronze 932 were calculated using experimental data obtained from this system. Reported values for these materials are known to be in the range of $207 \mathrm{GPa}$ and $103-124 \mathrm{GPa}$ at room temperature for $\mathrm{H} 13$ Tool Steel and Bronze 932 respectively [43]. Through this, it was determined that this system was capable of consistently performing material evaluation tests on samples at room temperature, Table 1 . With very little deviation between tests, this system was found to be an effective room temperature load-based instrumented indentation material characterization tool.

Table 1: Room temperature elastic modulus measurement.

\begin{tabular}{|c|c|c|}
\hline Material & H13 Tool Steel & Bronze 932 \\
\hline Literature & $207(\mathrm{GPa})$ & $103-124(\mathrm{GPa})$ \\
\hline Test 1 & 214.3 & 115.9 \\
\hline Test 2 & 207.5 & 106.8 \\
\hline Test 3 & 214.1 & 106.8 \\
\hline Test 4 & 209.1 & 110.2 \\
\hline Test 5 & 205.9 & 115.5 \\
\hline Average & 210.2 & 111.0 \\
\hline Deviation & 3.8 & 4.5 \\
\hline
\end{tabular}

Having now established the room temperature accuracy of this unit, elevated temperature micro-indentation tests were performed on two alloys: $\mathrm{H} 13$ Tool Steel and Haynes 230 nickel-based superalloy. Initial calibration tests were performed on a 1" x 1" x $1 / 4 " \mathrm{H} 13$ Tool Steel coupon at $22^{\circ} \mathrm{C}, 100^{\circ} \mathrm{C}, 200^{\circ} \mathrm{C}$, $300^{\circ} \mathrm{C}, 400^{\circ} \mathrm{C}$ and $500^{\circ} \mathrm{C}$. The results of these elastic modulus evaluation tests displayed good correlation to reported literature values, Figure 12 [43]. 


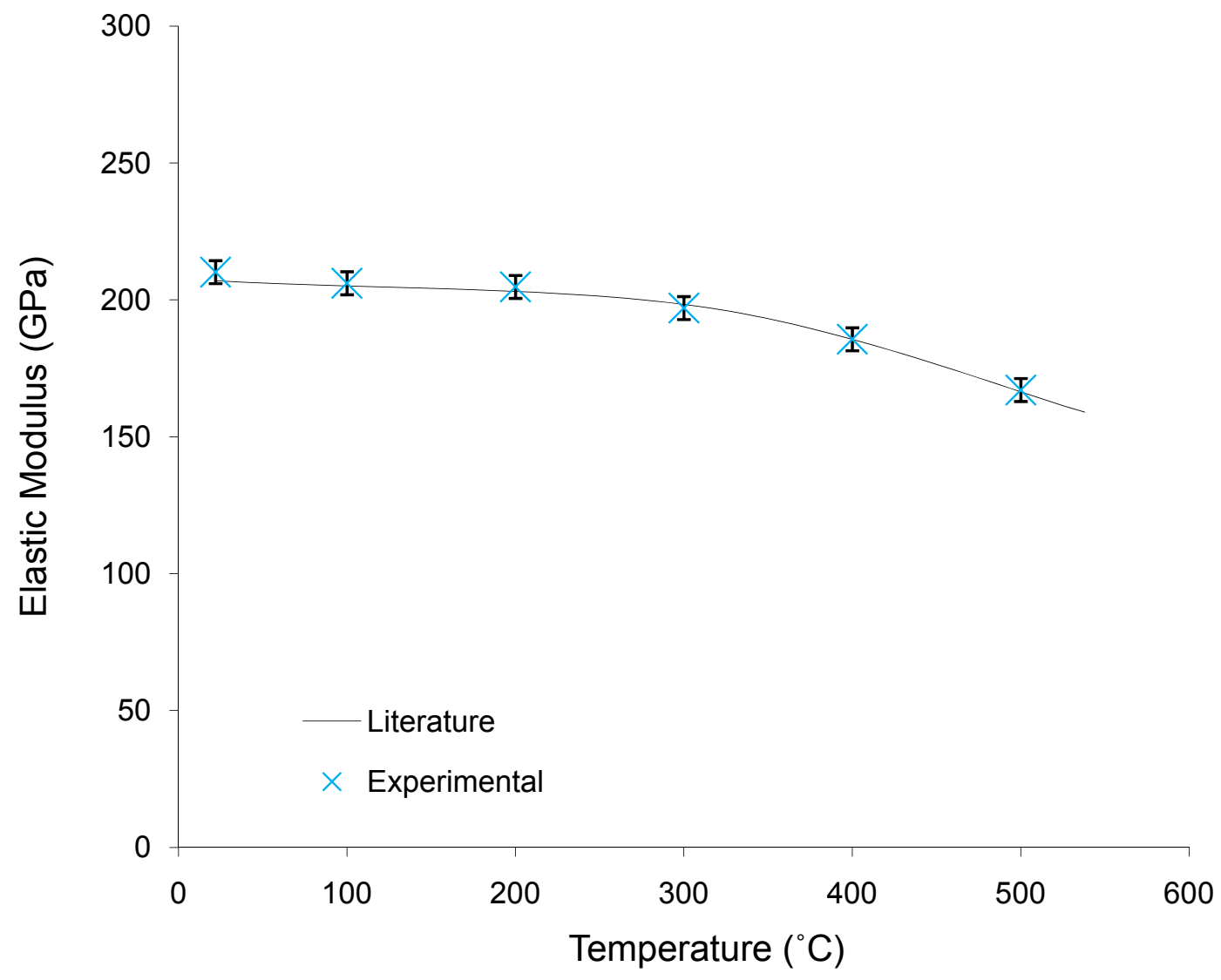

Figure 12: High temperature indentation elastic modulus results on an $\mathrm{H} 13$ tool steel coupon with \pm 1 standard deviation [43].

At all temperatures five multiple partial unloading micro-indentation elastic modulus evaluation tests were performed using a $2 \mu \mathrm{m}$ loading and $1 \mu \mathrm{m}$ unloading displacement. Although a total actuator displacement of $30 \mu \mathrm{m}$ was used throughout testing, the maximum load reached during each test decreased along increasing thermal load. This load reduction is the result of hardness decreases with increasing temperature of the H13 Tool Steel. Additionally, elastic modulus evaluation of a single crystalline Haynes 230 superalloy at $1000^{\circ} \mathrm{C}$ show good correlation with reported literature values, Figure 13 [44]. 


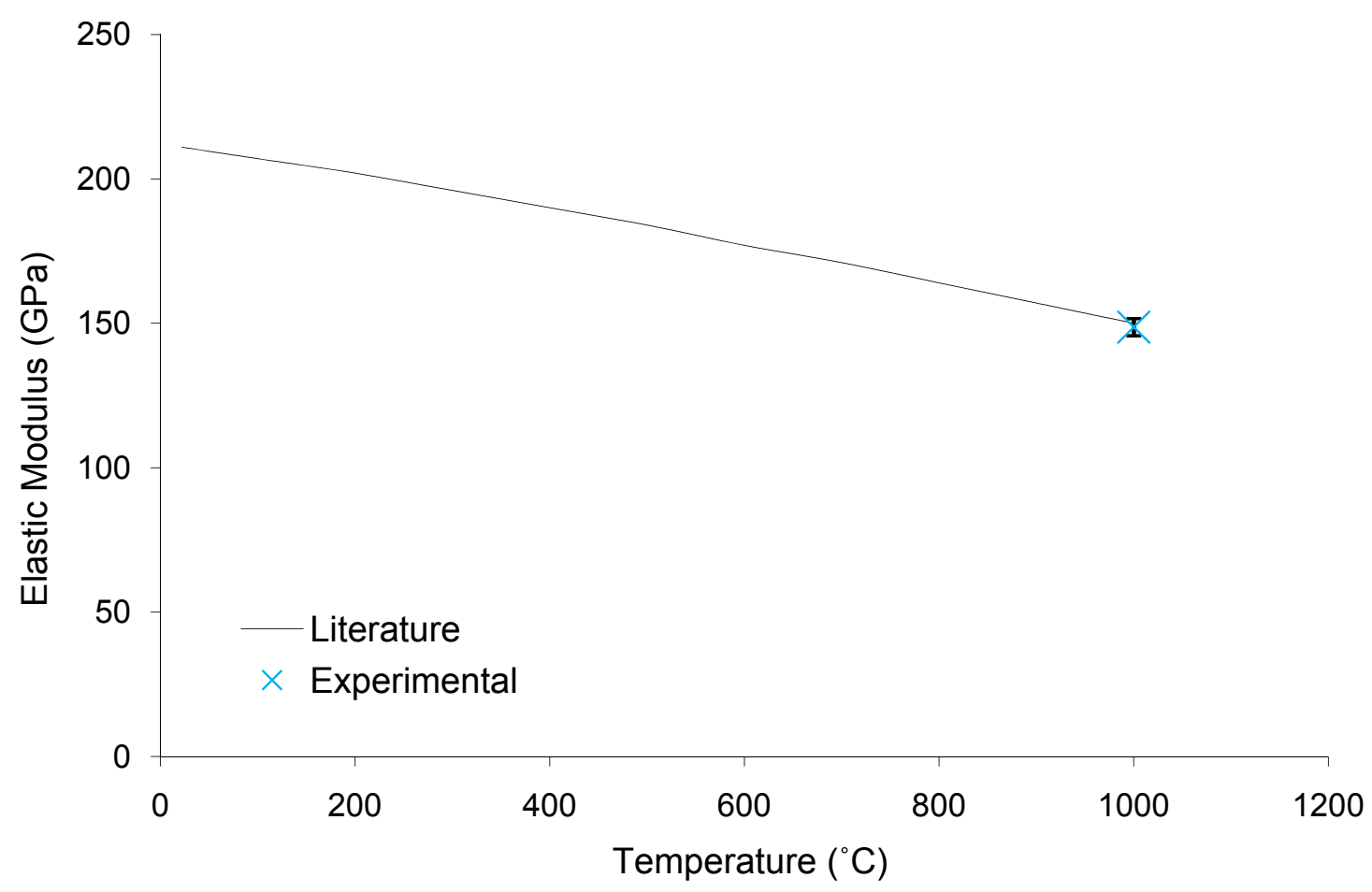

Figure 13: High temperature indentation elastic modulus results on a Haynes 230 coupon with \pm 1 standard deviation [44].

Also, indentation creep tests of Haynes 230 at $1200^{\circ} \mathrm{C}$ were found to be in agreement with known creep exponent data as well. Furthermore, while a thin oxide layer was found to form at these high temperatures, its effect on the materials overall elastic modulus is relatively low, Figure 14.

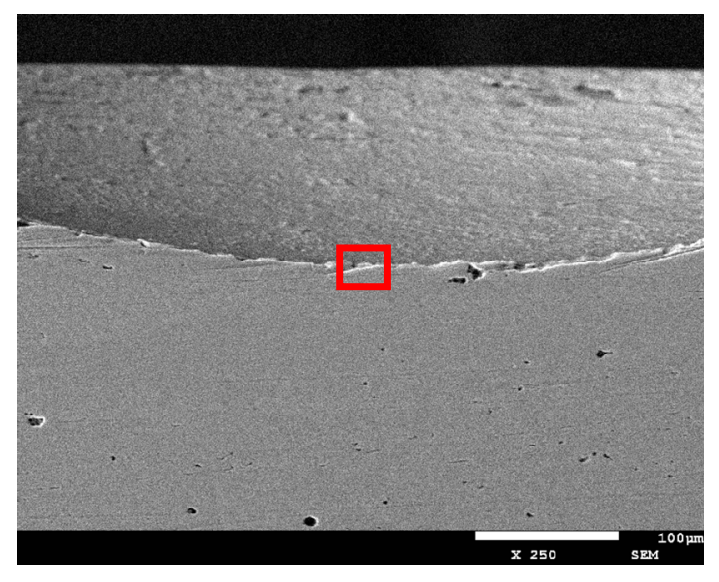

(a)

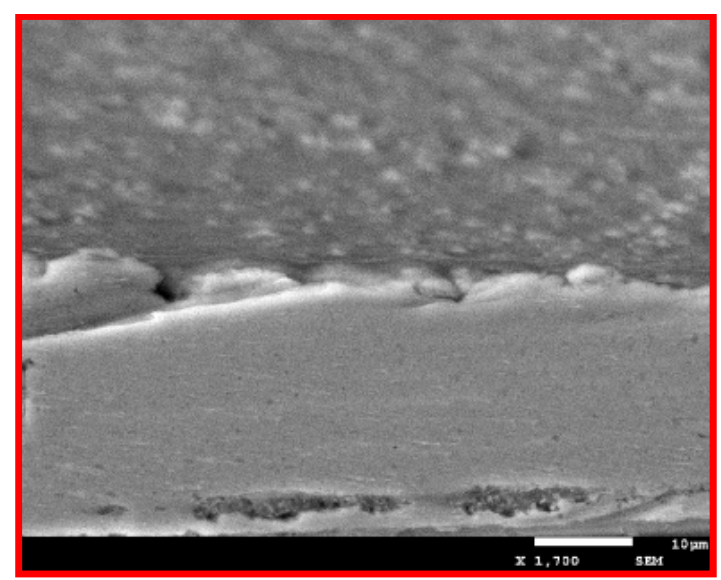

(b)

Figure 14: Iron oxide scales grown on $\mathrm{H} 13$ Tool Steel at $500^{\circ} \mathrm{C}$; a) x250 and b) x1700 
The relatively large loads $(60 \sim 90 \mathrm{~N})$ at which these micro-indentation tests were performed produces results that reflect only the sample's bulk rather than surface elastic modulus. Thus, effects of any thin oxide scales that may form during testing are only observed at low indentation loads. Furthermore, the thickness of the oxide layer formed is highly dependent on both temperature and environmental conditions. Consequently, as evaluation temperature increases, the maximum indentation load required to obtain bulk material properties must follow. Also, if a combined modulus is desired, total indentation depth must be reduced. Yet, recent studies have shown that although reasonable elastic modulus and hardness values for monolithic layers may be obtained, these tests are incapable of providing true modulus values of multilayered materials because of their inherent anisotropy [45]. Due to this, if these materials are to be evaluated at higher temperatures an inert gas environmental indentation system must be developed.

\subsection{Environmental High Temperature Indentation}

It is proposed that this technology be extended to the development of a high temperature micro-indentation test methodology for the performance evaluation of TBC systems in simulated oxy-fuel environments, Figure 15 


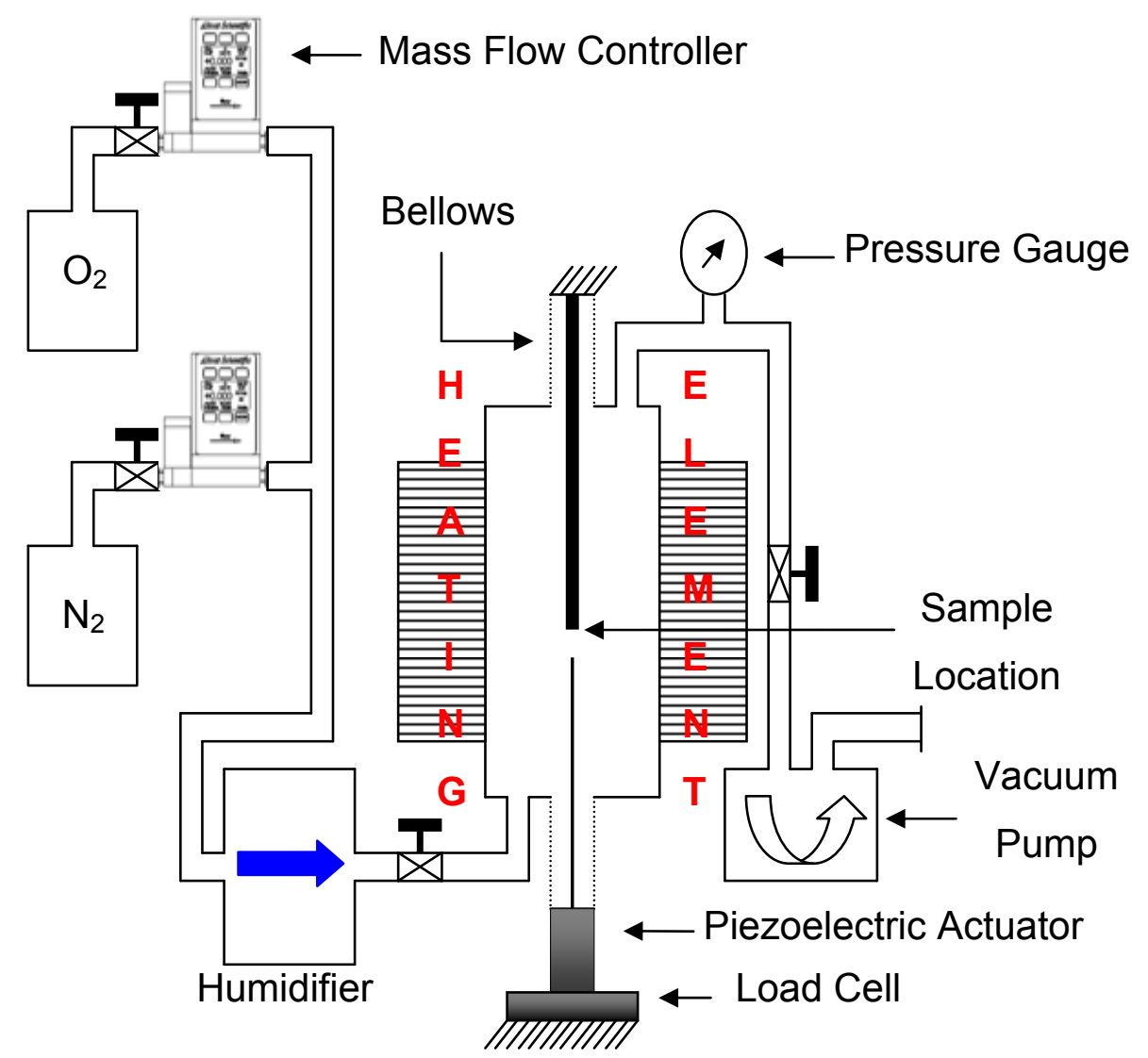

Figure 15: Controlled environment high temperature indentation system.

It is anticipated that with the development of this thermal flux indentation systems, TBC coupons will be evaluated in $\sim 50 \%$ steam/air environments at $1200^{\circ} \mathrm{C}$. In addition, at periodic intervals ( $\sim 100$ hour), these coupons will be removed from the furnace and examined for damage/spallation followed by further thermal loading until failure is reached. With this information it is believed that more sophisticated and accurate numerical TBC failure prediction models will be developed [46].

Developed through alteration of the previously presented high temperature micro-indentation system, the controlled environment high temperature indentation system requires the use of several additional components, Figure 16. 


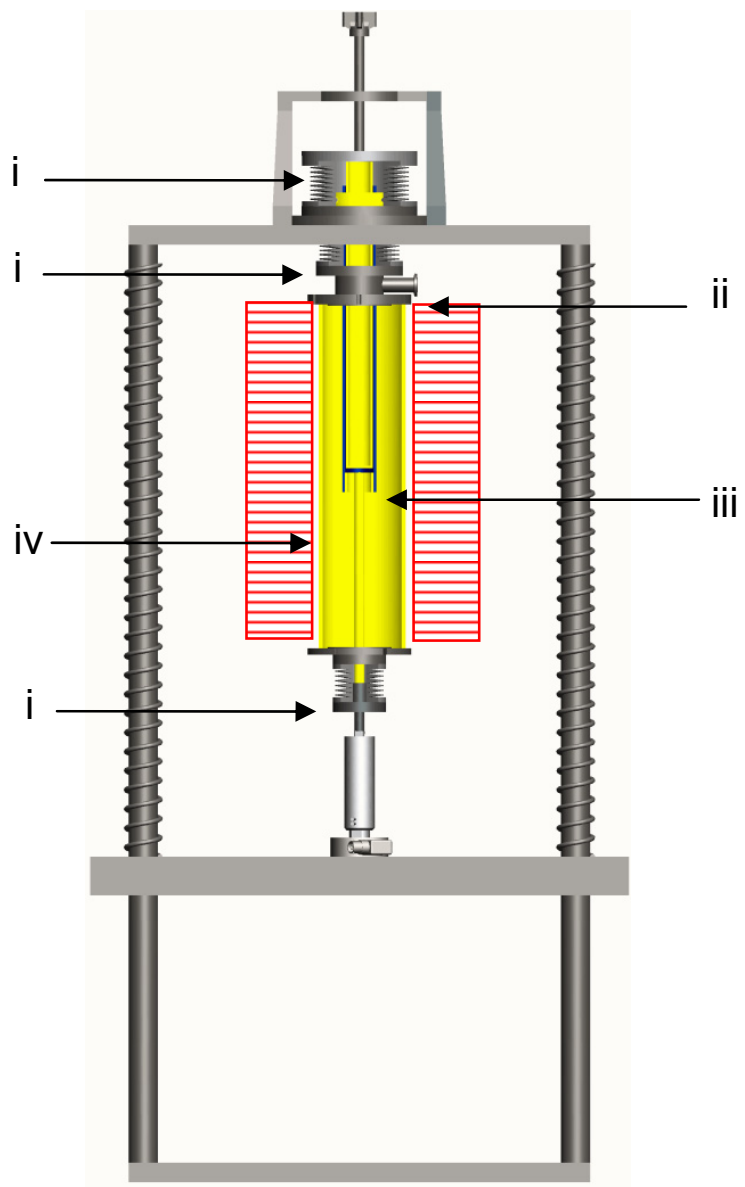

Figure 16: Controlled environment high temperature indentation system schematic; i) stainless steel bellows, ii) gas/moisture/vacuum port, iii) sample (shown schematically in Figure 11) and iv) cylindrical alumina tube.

Although the evaluation chamber requires vacuum capability, indentation chain components must not be exposed to harsh environmental conditions and thus should remain external, thus a series of bellows is utilized to allow actuator to indenter displacement but prevent environmental damage of the indentation chain. Furthermore, losing its mechanical strength at temperatures above $\sim 500^{\circ} \mathrm{C}$ [47], stainless steel requires external heat removal while under thermal loads above this level. Consequently, an external cooling system is required to prevent both oxidation and lose of mechanical strength of several system components at elevated temperatures, Figure 17. 


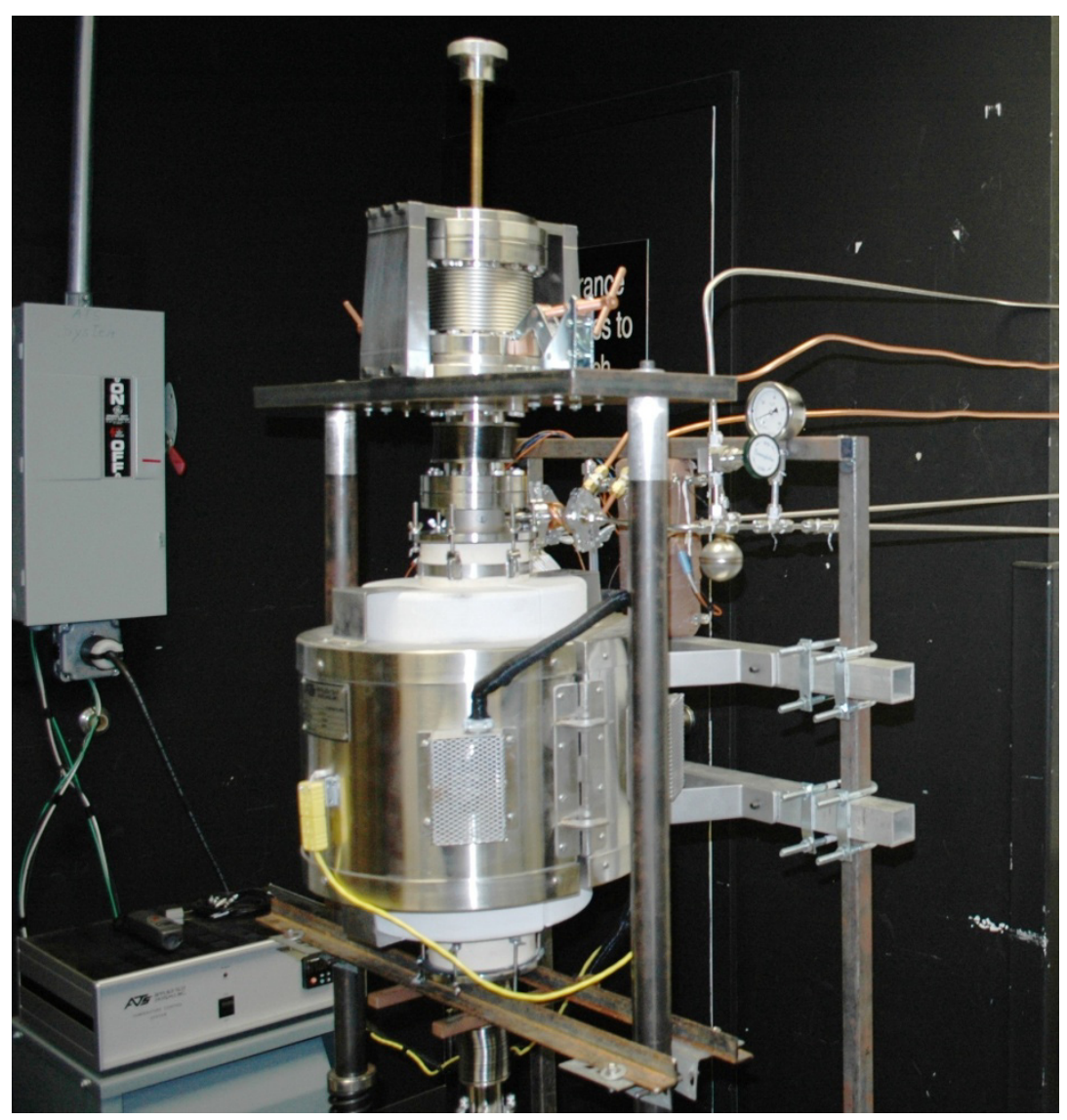

Figure 17: Controlled environment high temperature indentation system, gas/steam and water cooling lines.

The addition of this heat exchanger is purely external and does not alter the overall design of the controlled environment high temperature indentation system. Additionally, a more environmentally resistant thermocouple has been utilized to prevent both oxidation and degradation of the sensor's junction during the entertainment of moisture in the evaluation chamber.

Composed of stainless steel, the inlet inert gas line is initially fed through a liquid humidifier where it is first saturated with steam prior to entering the thermal chamber. Although the gas bottle is under a positive pressure, differential losses through the gas/steam line require the chamber first be brought to a vacuum state prior to the introduction of steam. Thus, a vacuum pump has been installed and will allow the thermal chamber to be purged multiple times to achieve a steady-state gas/steam state prior to thermal loading, Figure 18. 


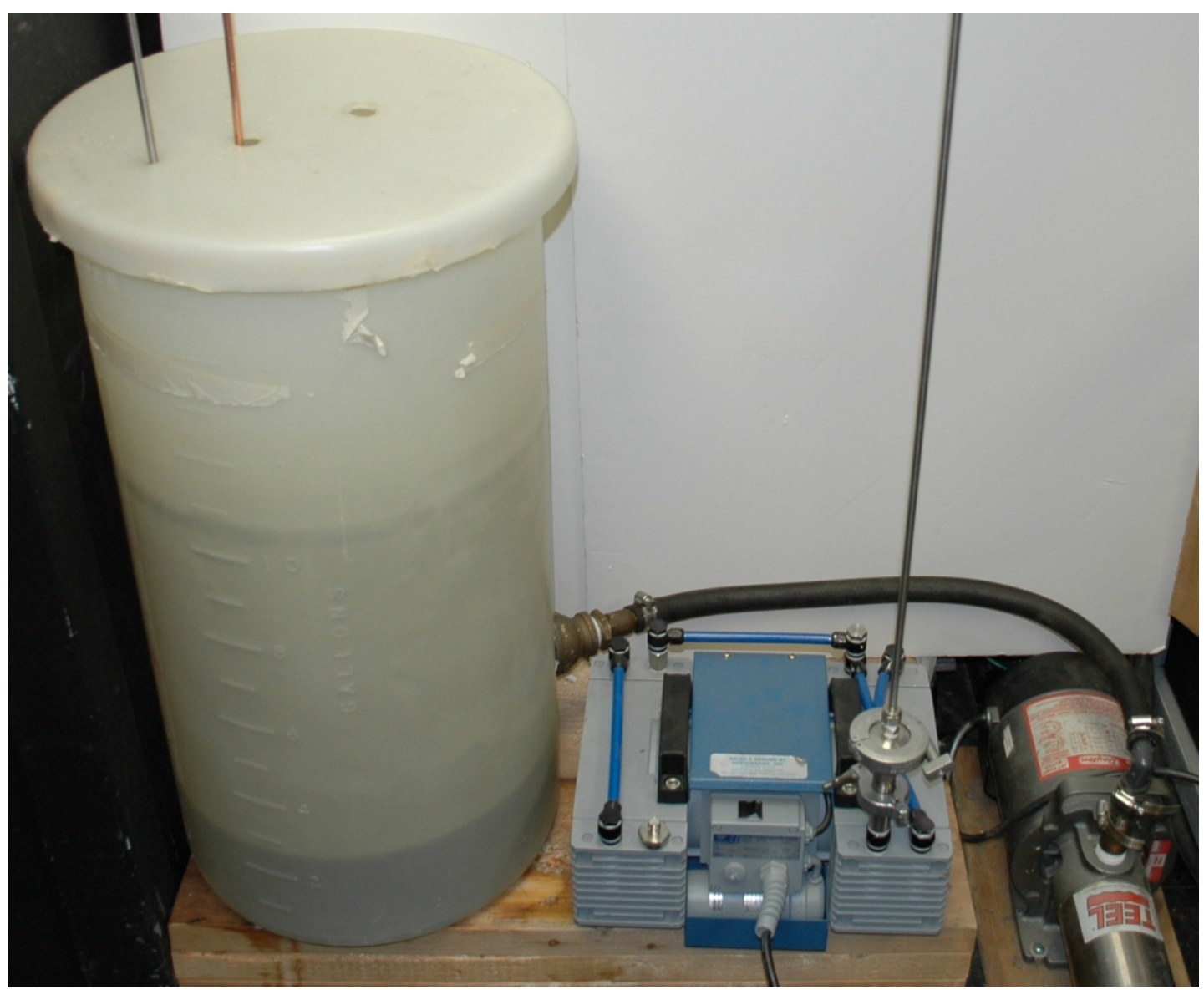

Figure 18: Controlled environment high temperature indentation system, external water pumps and cooling tank.

This vacuum purging procedure is repeated until a desired level of steam and inert gas mixture within the chamber is reached. In order to control the humidity content, a series of thermal pads are applied on the gas/steam lines leading to the thermal chamber. A total of three thermal pads have been installed, all of which are powered through a controller placed at the bottom of the indentation system, Figure 19. 


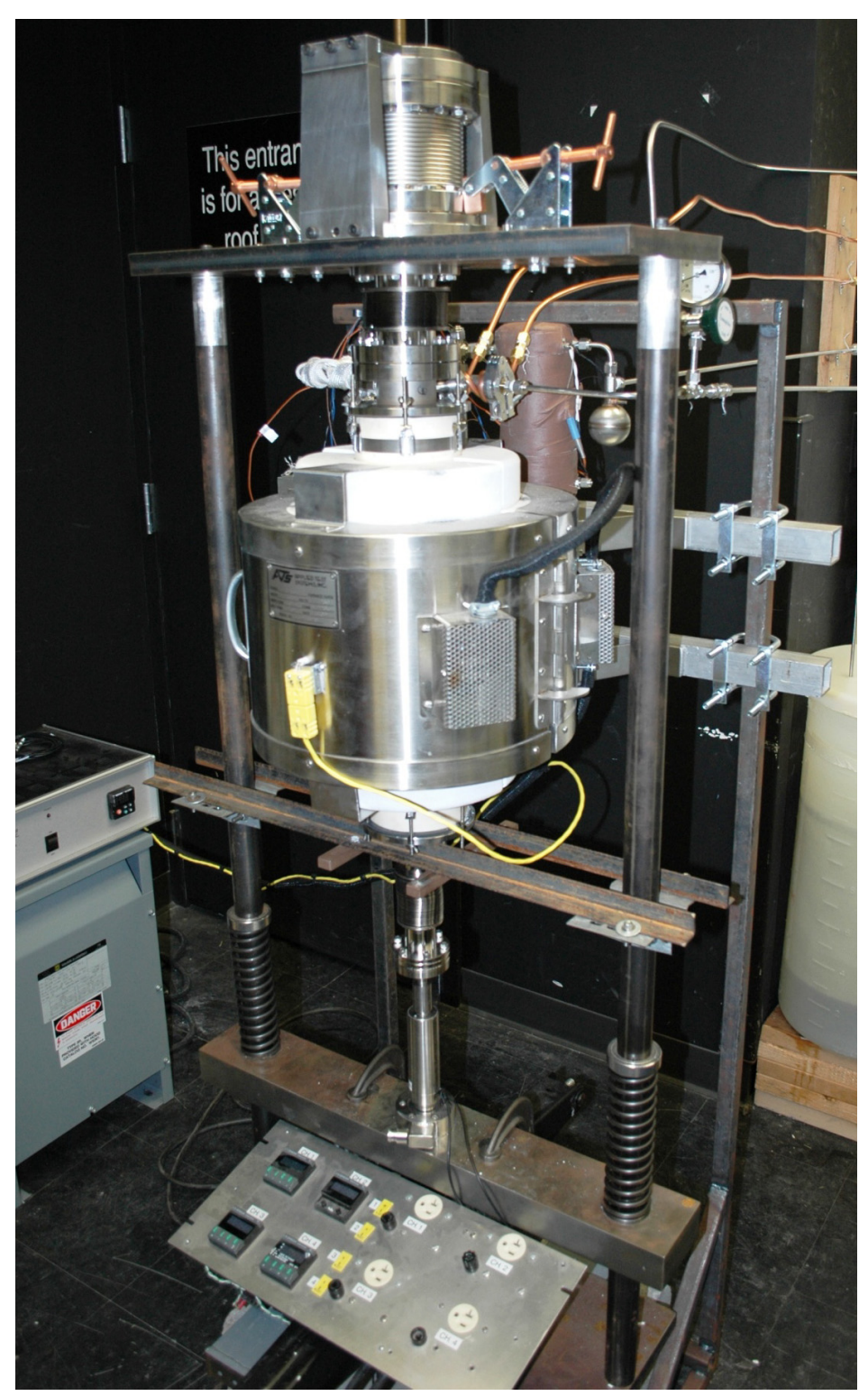

Figure 19: Controlled environment high temperature indentation system; overview.

Warming of the lines between the humidifier and the thermal chamber will ensure no water vapor condensation will occur prior to reaching the preheated sample chamber. Once a sufficient level of saturated steam exists in the thermal chamber, all outlets are sealed and the furnace is then brought to the desired testing temperature. This increase in temperature will cause pressures within the chamber to increase, thus a 1PSI poppet check valve is installed to release all additional gases exhausted by the system. With the completion of the inert 
gas/controlled moisture content high temperature environmental chamber, initial simulated TBC thermal cycling has been conducted, Figure 20.

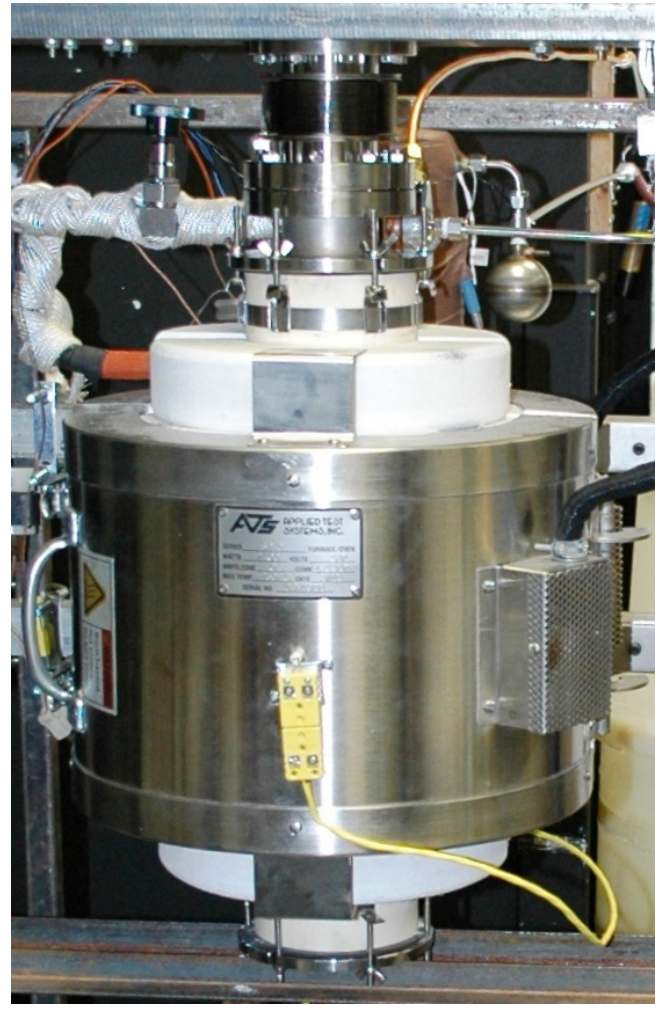

(a)

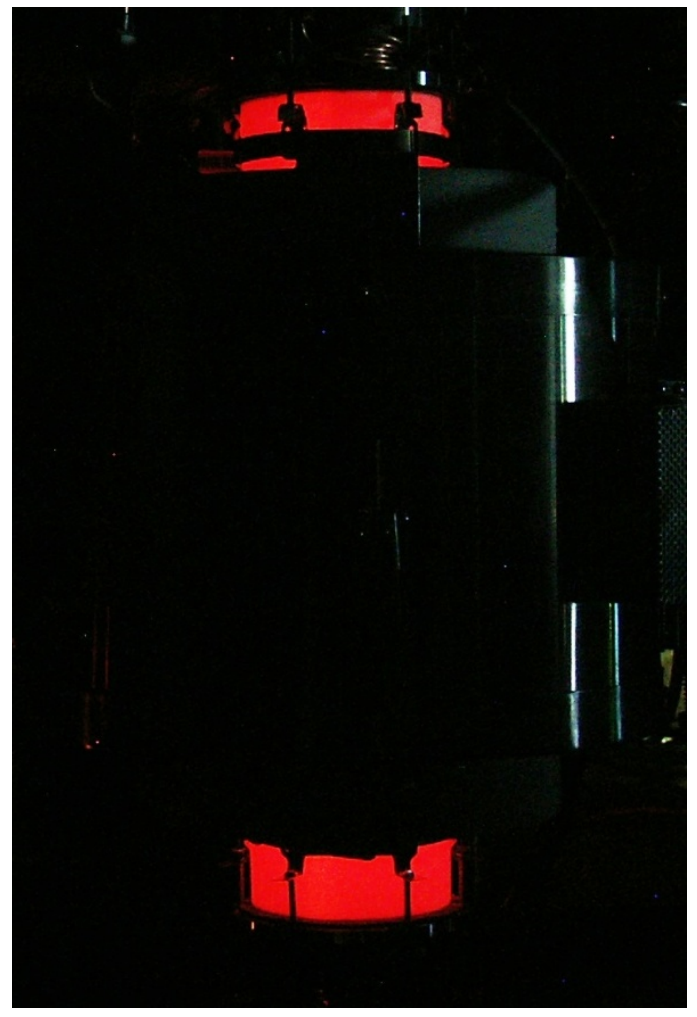

(b)

Figure 20: Controlled gas high temperature indentation system at a) room temperature and b) $1100^{\circ} \mathrm{C}$. 


\section{Chapter 3: Thermal Barrier Coatings}

Nickel based super alloys with protective thermal barrier coatings (TBC) are widely used systems for land based gas turbine engine applications. These coating are efficiently capable of extending the life and firing temperatures of combustor and stationary turbine components [47]. Currently, 6-8 weight percent yttria partially stabilized zirconia (YSZ) TBCs have facilitated the use of superalloys at temperatures $100 \sim 300^{\circ} \mathrm{C}$ higher than that of conventional operation $[48,50]$. However, the effectiveness of these TBC systems is limited by their structural reliability, corrosion resistance and unpredictable failure. Composed of multiple layers, the top coat or thermal barrier of the TBC matrix, also protecting the system from oxidation is deposited with a metallic layer residing between it and the super alloy substrate. This bond coat (BC) is often comprised of nickel, cobalt, and chromium with sufficient concentrations of aluminum ensuring the later developing thermally grown oxide (TGO) is predominantly composed of stable $\alpha-\mathrm{Al}_{2} \mathrm{O}_{3}$. Rare earth metals such as yttrium and platinum are also added in small amounts to facilitate adequate TGO adhesion [51]. The TGO, a major contributor to interfacial thermo mechanical resistance of the coating, although mainly composed of $\alpha-\mathrm{Al}_{2} \mathrm{O}_{3}$, entertains spinel oxides and other impurities such as yttrium and zirconia as well [52]. Each layer provides a specific material property needed by the TBC ensuring proper effectiveness and life. The turbine blade substrate, which is often air cooled internally by channels, establishes a large temperature gradient across its thickness. This super alloy is cast in single crystal or poly crystalline form composed of nickel or cobalt superalloys. It may also contain as many as twelve additional elements further enhancing specific material properties such as melting point, strength and oxidation resistance. At high operation temperatures, diffusion of these elements between the substrate and BC may occur. Furthermore, these diffusing elements can occasionally be found in the thermal growth oxide and ceramic top coat as well. This diffusion will have a profound 
influence on spallation failure of the thermal barrier coating, making it essential to treat this system as a material whose properties change over time.

\subsection{Yttria Stabilized Zirconia}

Few materials are able to perform the tasks required of thermal barrier coatings; however zirconia's low thermal conductivity, high thermal expansion coefficient and outstanding mechanical strength make it ideal for use in TBC systems. Yet, pure zirconia undergoes a polymorphic phase transformation upon heating and cooling, Figure 21 [53].

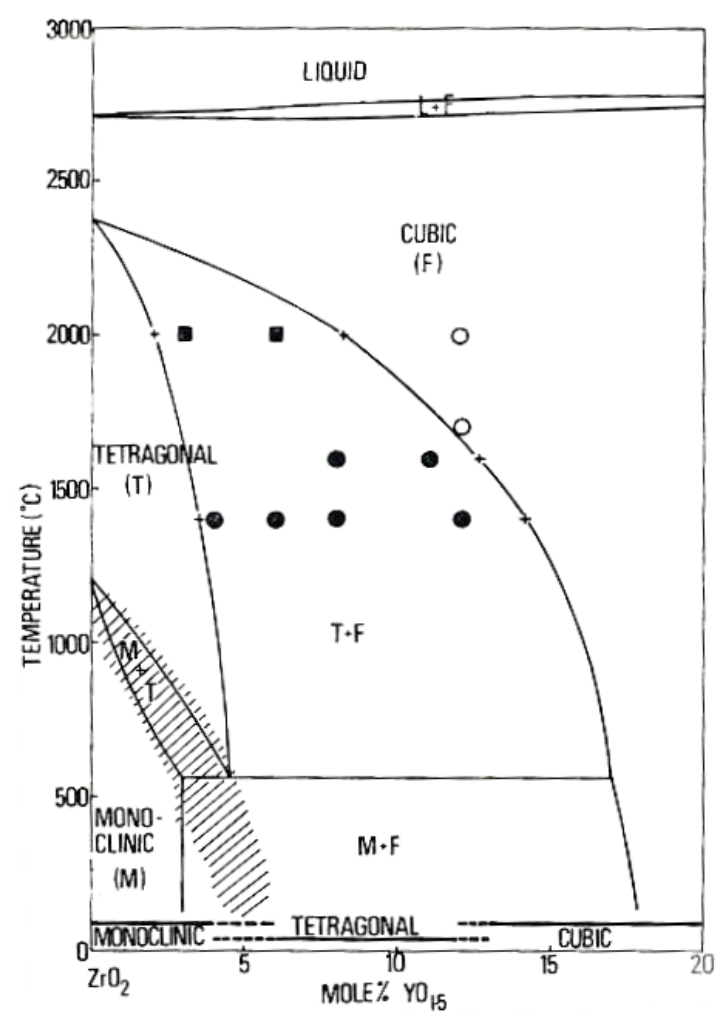

Figure 21: Yttria stabilized zirconia phase diagram [53].

This tetragonal to monoclinic phase transformation is martensitic as it does not require diffusion and occurs by small displacements of atoms within its structure. The transformation that occurs upon cooling initiates a relatively large volume increase and induces a significant amount of shear strain affecting its structural integrity. However, doping zirconia with other oxides such as yttria $\left(\mathrm{Y}_{2} \mathrm{O}_{3}\right)$ inhibits such a phase transformation and eliminates volume change [54]. 
This in turn enhances overall durability of the zirconia coating when exposed to thermal cycle fatigue.

As part of a continuing effort to develop advanced gas turbine engine components, YSZ layers containing 4 24.4 weight percent yttria were investigated [55]. Evaluations performed in a harsh, high temperature turbine like environments found YSZ of 6.2 7.9 weight percent yttria to be superior in life time fatigue to that of other percentages. Due to its high melting point, zirconia cannot be vaporized and subsequently deposited by conventional application processes. As a result, researchers turned to air plasma sprayed (APS) and electron beam physical vapor deposition (EB-PVD) techniques. These techniques although accomplishing a similar task form quite unique microstructures with the deposited YSZ. Additional details regarding both techniques are presented in later sections. Yet, regardless the application procedure, the YSZ ultimately provides thermal protection for both the superalloy substrate and the oxidative protective bond coat. This need for enhanced thermal protection has lead to the development of YSZ coatings as thick as $1 \mathrm{~mm}$. Although this increase in thickness does provide additional protection from the harsh environment of land based gas turbines, coating thicknesses have been found to reduce relative coating durability, Figure 22 [56].

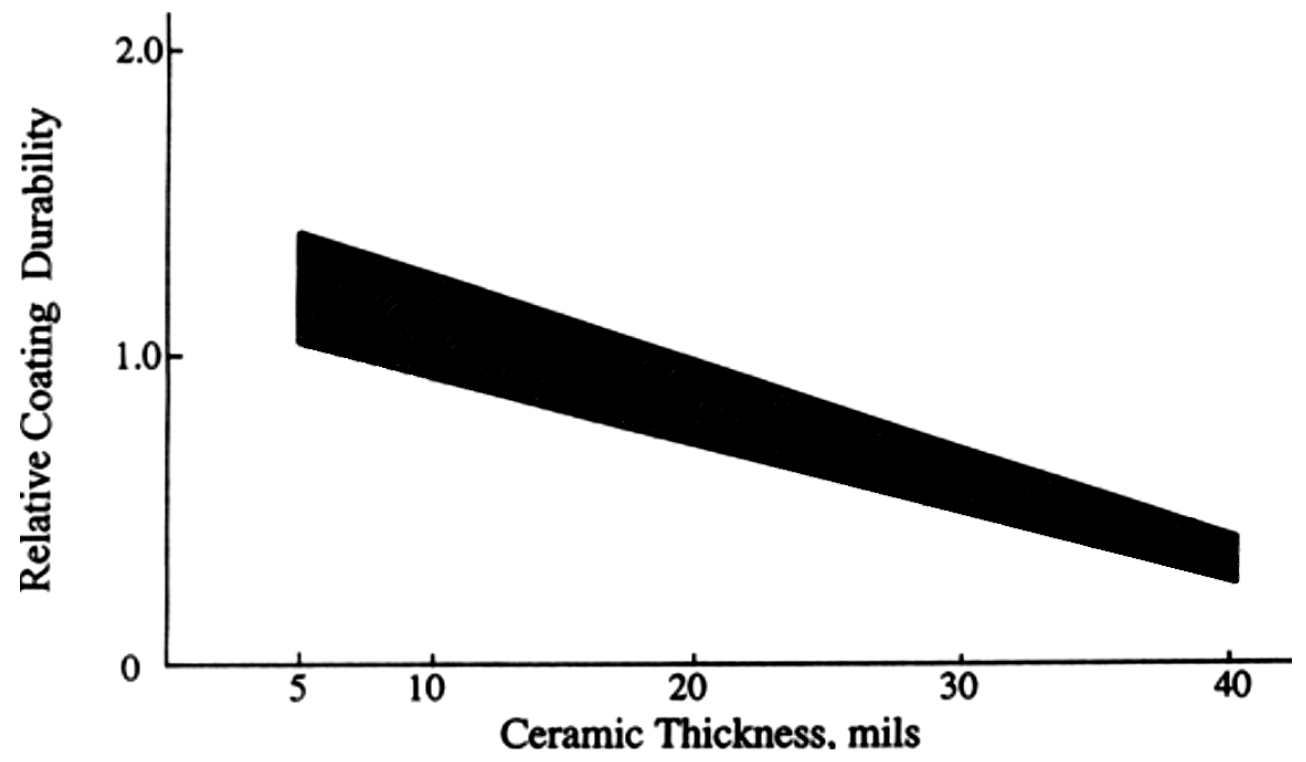

Figure 22: Reduction in relative coating durability with increasing thickness [56]. 


\subsection{Bond Coat}

Refractory metal bond coatings are necessary to create good adherence between the ceramic top coat and the substrate superalloy [57]. The bond coat (BC) does not only provide good adhesion for the ceramic top coat but also corrosion resistance for the superalloy substrate by developing aluminum oxide scales [58]. The porous nature of YSZ provides paths for oxygen ingress and subsequent oxidation of this layer. Through this process, the bond coat essentially dictates the spallation of the thermal barrier coating. As a result, an oxidation resistant metallic alloy is employed producing only the desired aluminum oxide $\alpha-\mathrm{Al}_{2} \mathrm{O}_{3}$. This alloy, often consisting of a MCrAlY (where $\mathrm{M}$ is $\mathrm{Ni}$ and/or Co) base can be applied using either air plasma sprayed (APS) or by electron beam physical vapor deposition (EB-PVD) techniques.

Generally, bond coat materials soften above $600^{\circ} \mathrm{C}$, thus if mechanical loads build up above these temperatures, extended creep behavior may be observed [59]. To improve these properties, the addition of reactive elements such Rhenium, Tantalum and Platinum in small amounts have produced bond coats with enhanced coefficient of thermal expansion coefficients, creep resistance, yield strength and strain properties have been developed leading to an overall extension of TBC life [60,61]. Yet more recently, a study of free standing $\mathrm{NiCoCrAl}(\mathrm{Y} / \mathrm{Hf})$ bond coats showed that variation of oxygen partial pressure during vacuum plasma spraying and vacuum heat treatment significantly affects the yttrium and hafnium distribution in the coating [62]. Where using high bond coat application partial pressures resulted in the yttrium and hafnium being mainly tied up in oxide precipitates. This effect is apparently responsible for an early alumina scale spallation and premature failure of the TBC system during cyclic oxidation. In contrast, coatings sprayed at low partial pressures revealed an overdoping effect, thus extensive yttrium and hafnium incorporation into the scale were observed resulting in an accelerated scale growth rate and internal oxidation. Due to this, these parameters must be optimized to develop the most reliable bond coating application procedure possible. Furthermore, coefficients of thermal expansion mismatch strains should 
be minimized when choosing the appropriate bond coat for any thermal barrier system; this will reduce the stresses and strains primarily responsible for spallation failure of these coatings [63].

\subsection{Thermally Grown Oxide}

Arguably the most important layer of the TBC matrix, responsible for the majority of spallation failures in thermal barrier coatings, the thermally grown oxide is found just below the ceramic top coat. Although, the generation of TGO is inevitable, the ideal bond coat is designed to ensure its growth is slow, uniform, and defect free. Such an oxide scale has low oxygen ionic diffusivity and provides an excellent protective barrier, suppressing further bond coat oxidation. Although predominantly composed of $\alpha-\mathrm{Al}_{2} \mathrm{O}_{3}$, initially the $\mathrm{TGO}$ is found to be an amorphous $\theta-\mathrm{Al}_{2} \mathrm{O}_{3}$, soon thereafter converting to the more stable and desirable $\alpha-\mathrm{Al}_{2} \mathrm{O}_{3}$. The TGO morphology is such that oxygen diffusion through the aluminum grains is faster than that of aluminum diffusion; this causes further oxide growth to occur at the structures base leading to an upward growth mechanism. The presence of large elongated grains near the TGO/Bond Coat interface indicates that additional oxide growth occurs predominantly at this region [64]. This also indicates that the oxygen diffuses through the $\mathrm{Al}_{2} \mathrm{O}_{3}$ layer is much faster than the aluminum. The relatively rapid inward diffusion of oxygen and slow outward diffusion of aluminum is responsible for the formation of three distinct layers within the TGO. The first, an $\mathrm{Al}_{2} \mathrm{O}_{3}-\mathrm{ZrO}_{2}$ mixed zone followed by a well developed and crystallized $\mathrm{Al}_{2} \mathrm{O}_{3}$, and finally a fined grain $\mathrm{Al}_{2} \mathrm{O}_{3}$ layer mixed with spinel oxides $\mathrm{Ni}(\mathrm{Cr}, \mathrm{Al})_{2} \mathrm{O}_{4}$ [65]. These three layers, along with defects, voids, dislocations, and yttrium segregations make up the thermal barrier coating thermally grown oxide microstructure, Figure 23 [52]. Furthermore, forming only after the BC in sufficiently depleted of its aluminum reservoir, complex oxides of both yttrium and aluminum (garnet) or nickel and cobalt (spinel) form [52]. 


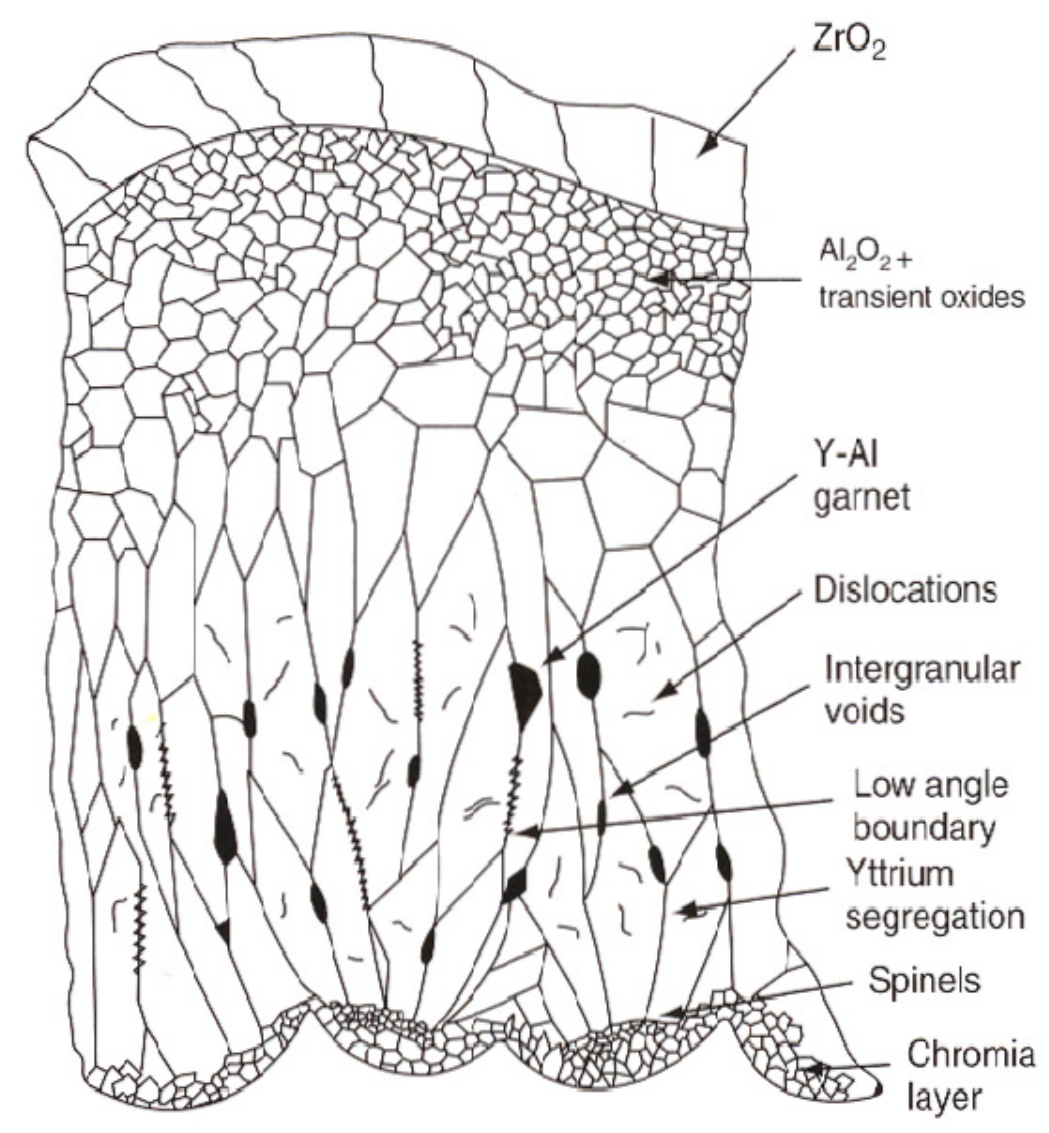

Figure 23: Thermally grown oxide microstructure [52].

These TGO characteristics and growth rate are largely controlled by the bond coat microstructure and microchemistry. Although relatively thin, the energy stored in this layer is relatively large. This contributes significantly to the potential for delaminations between the bond coat and TGO. Additionally, TGO growth involves counter diffusion of oxygen and aluminum along the $\alpha-\mathrm{Al}_{2} \mathrm{O}_{3}$ grain boundaries. Therefore, the growth rate of the TGO is fundamentally dependent upon this oxygen and aluminum diffusion rate through the $\alpha-\mathrm{Al}_{2} \mathrm{O}_{3}$ grain boundaries and is dictated by grain sizes. To accommodate further $\alpha-\mathrm{Al}_{2} \mathrm{O}_{3}$ formation within the existing grain boundaries, lateral deformation within the TGO creates a compressive growth stress, however these are intrinsic stresses and are due to chemical reactions and phase transformations [66]. This along with thermally induced stresses caused by material cooling are responsible for a majority of the ratcheting/rumpling that occurs within this layer [67]. Additionally, oxidation rates can be influenced by temperature, bond coat reactive element 
content, surface morphology and YSZ thickness, yet alumina scale growth rates of MCrAlY bond coats have been frequently observed to follow sub-parabolic oxidation kinetics with a time exponent approaching 0.33 [68]. This sub parabolic, near cubic, kinetics has been quantitatively correlated with the growth mechanisms and columnar microstructure of the alumina scale [69]. This was accomplished under the assumption that the oxide grains provide a short-circuit path for the inward diffusion of oxygen, thus also allowing any additional growth to occur from the base of this layer. This growth mechanism also forces the increase in lateral oxide scale grain width to become a linear function of depth from the surface. This width increase ultimately results in a reduction of the effective oxygen diffusion coefficient and consequently near cubic kinetics.

Recent EB-PVD TBC oxide scale growth observations have shown that those containing physical defects are capable of maintaining good TGO TBC adherence in spite of enhanced growth rates compared to thin, defect free scales. To accomplish this, minor elemental additions to the BC such as zirconia $(\mathrm{Zr})$ result in the formation of a non-uniform TGO with a number of cracks near its interface with the YSZ. This enhanced oxide growth rate and cracking allow the lifetime of the TBC system to increase by approximately twice that of Zr-free BCs [70]. This TBC lifetime extension with the $Z r$ doped $B C$ in related to the specific non-uniform scale morphology, which impedes crack propagation at the TGO/BC interface. Similar effects on the TGO morphology were also observed when hafnium ( $\mathrm{Hf})$ was present in the $\mathrm{BC}$ as well [71]. It is important to note that the positive effects of $\mathrm{Hf}$ and/or $\mathrm{Zr}$ on the TGO morphology and associated TBC lifetime crucially depend on the distribution of these reactive elements in the coating. This distribution in turn is strongly affected by the manufacturing parameters of the MCrAlY coatings.

\subsection{Superalloy Substrate}

Many of the super alloys used in hot section components of gas turbine engines are of a nickel base. The development of these alloys began in the late 1920 s as the need for more creep resistant materials for jet engines became a 
necessity [72]. Throughout the late 1940s, with the addition of molybdenum as a solid solution and carbide forming strengthener, these alloys were subsequently used in forging many turbine blades and buckets. By the late 1950s operating temperatures of gas turbines were limited by the capability of available wrought nickel base alloys, further these were costly and not easily forged. To meet this demand manufactures directed their attention to investment cast nickel base super alloys. The microstructure of these investment cast nickel base superalloys consists of a face centered cubic (FCC) solid solution matrix containing carbides and the coherent intermetallic phase $\gamma^{\prime}$. Strengthened by the additions of aluminum, titanium and tantalum, these elements when combined with nickel form the FCC $\gamma^{\prime}$ of which the alloy is composed. Also contributing to the alloys overall strength, additions of elements in solid solution such as molybdenum, tungsten and chromium improve temperature, oxidation and hot corrosion resistance. Furthermore, cobalt, aluminum chromium and titanium are often added to these alloys in trace amounts. With the addition of such materials cast nickel base super alloys provide a combination of high temperature capability, strength, ductility, oxidation resistance, microstructural stability, hot corrosion resistance and castability required for turbine blade forging.

In the 1980s the chemistries for the first nickel base superalloys suited for single crystal casting were derived from that of the aforementioned investment cast compositions. Designers of these single crystal nickel base super alloys later succeeded in optimizing the mechanical properties of these alloys by introducing large amounts of tungsten, tantalum and molybdenum. With the introduction of rhenium, a major step was taken in the increase of mechanical strength of theses alloys but at the expense of density, castability, cost and micro structure [73]. Since the development of single crystal technology for nickel base super alloys, turbine blade temperature capability has significantly increased. Yet ultimately this layer remains structural and relies on thermal barriers composed of YSZ for protection against the harsh environments of which hot section land based gas turbines. 


\subsection{Application}

The method by which the YSZ and BC are applied is the primary cause for the ways in which these thermal barriers eventually fail. The two primary process used for TBCs include both electron beam physical vapor deposition (EB-PVD) and air plasma sprayed (APS), Figure 24.

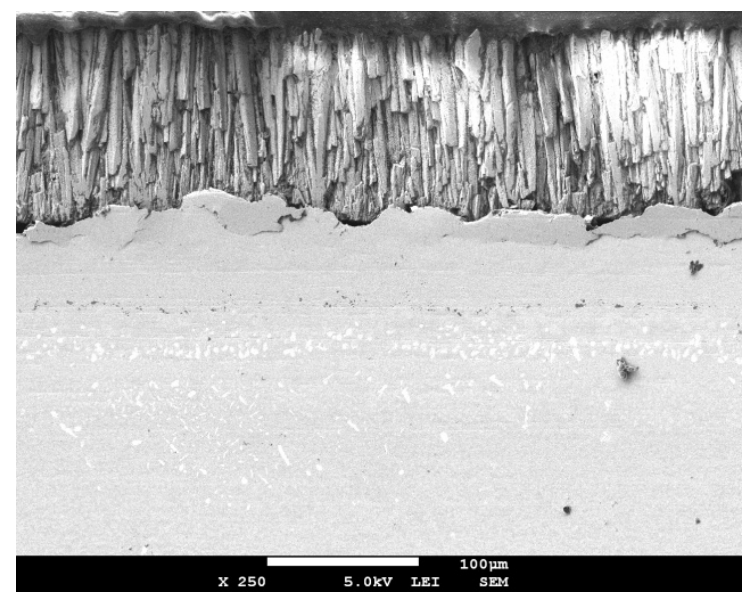

(a)

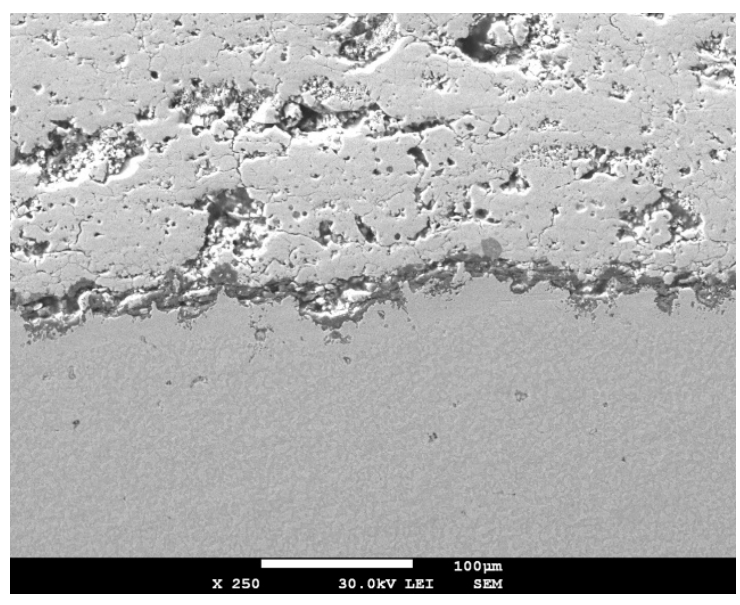

(b)

Figure 24: TBC Cross-sectional SEM Micrograph, a) EB-PVD and b) APS.

The basic principle of which the EB-PVD process is preformed enables the development of a columnar structure within the YSZ coating. Yet, that of the APS creates a series of randomly oriented layered splats. With a higher processing cost, the EB-PVD application procedure requires that a melt pool of raw YSZ particles be created in an evacuated chamber via a focused high-energy electron beam. As this molten pool evaporates, the substrate in held above after which the coating forms by deposition of vapor molecules. The coating that forms is more dense and thermal resistant than that of APS coatings, yet the cost of coating numerous large turbine blades has prevented this coating procedure from becoming prevalent for land based power generation applications. Due to this the APS process has become much more widespread in this industry. In this process, zirconia powders are fed into the hottest section of a high pressure plasma jet. The high temperatures at which the plasma flame burns causes the zirconia powders to be come molten and accelerated toward the target. Upon cooling, the zirconia particle once again becomes solid and is now bonded to the 
substrate. This interlocking between the zirconia splat and substrate is considered to be primarily mechanical in nature. Although more pores than that of the EB-PVD, APS TBCs provide adequate thermal protection when applied to hot section components of land based gas turbines.

\subsubsection{EB-PVD Microstructure}

As the coating applied in this process is highly dependent on the composition of the vapor to which it is exposed, it is important that properties of the ingot be quite pure. When vaporizing a single ingot often multiple materials are melted, if these species have widely differing vapor pressures, it is that of the highest vapor pressure which makes up the vast majority of the subsequent coating. Thus, the ingot composition must be adjusted to achieve the desired coating chemistry. Regardless the composition, the EB-PVD coating is characterized by its columnar microstructure, Figure 25 [74].

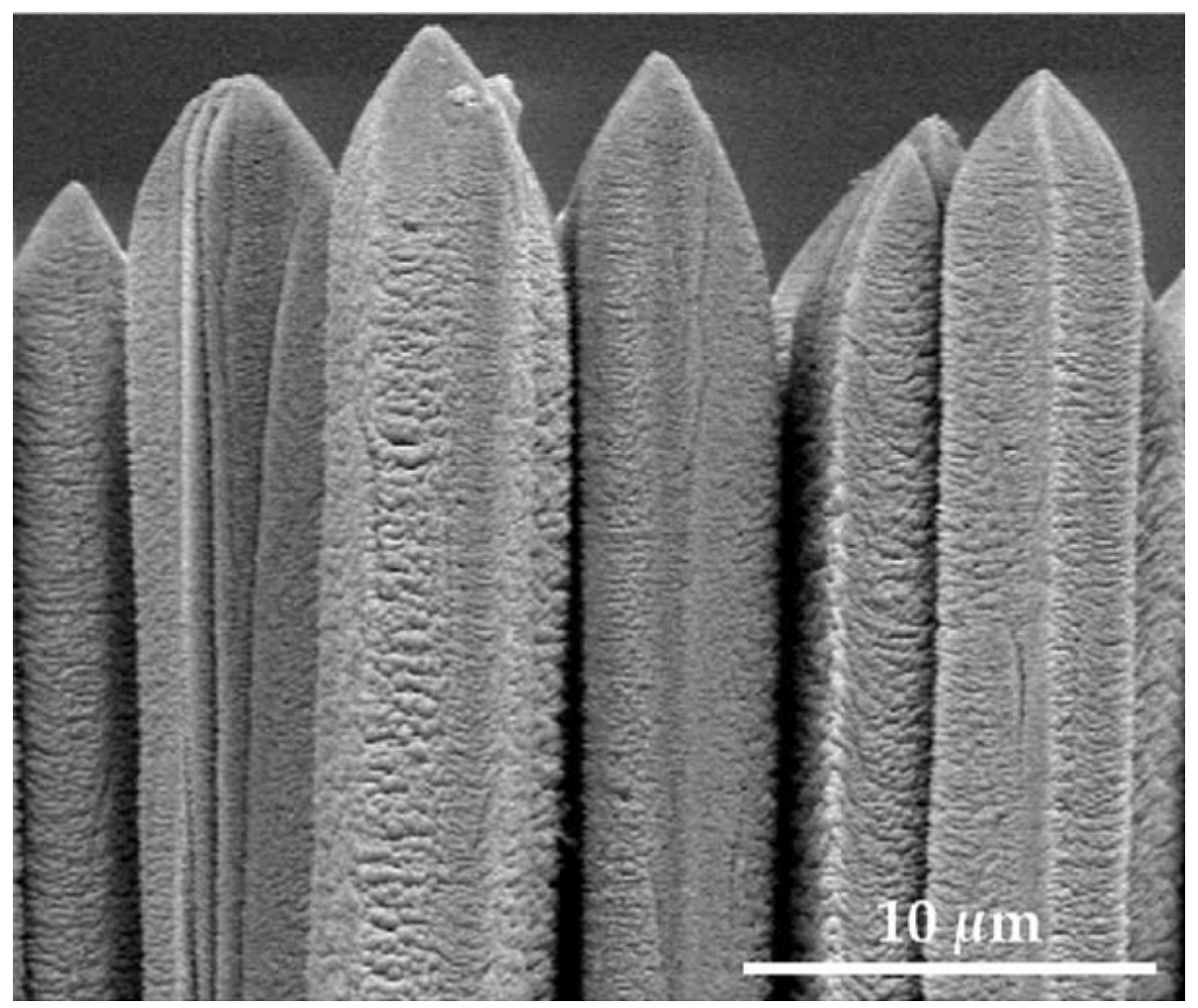

Figure 25: EB-PVD columnar structure formed upon application [74].

As vapor molecules approach the substrate with the help from their thermal energy, these molecules are absorbed by a surface diffusion mechanism 
while seeking low energy sites. Once attached to the surface these molecules now form nuclei and grow as additional molecules are deposited. Whether the subsequent depositing molecules will form new nuclei or contribute to the growth of existing nuclei is dependent on processing parameters such as chamber pressure and temperature. The deposition of a fresh layer of molecules, followed by diffusion and the ensuing energy exchange, leads to the development of a columnar growth mechanism. As these neighboring columns have weak intercolumnar bonding, this coating technique is able to produce the strain tolerant structure required of a TBC systems.

\subsubsection{APS Microstructure}

The unique microstructure that is produced via the plasma spray process typically yields a coating material with 10 to 15 percent porosity per volume, yet this value is dependent on processing parameters such as particle size and spray distance. In this process, zirconia particles are injected into a plasma melt. While passing through the plasma plume, these now molten particles are then projected towards and eventually impact a roughened substrate surface. Upon impact, the deposited particles partially flatten thus forming splats, Figure 26 [75].

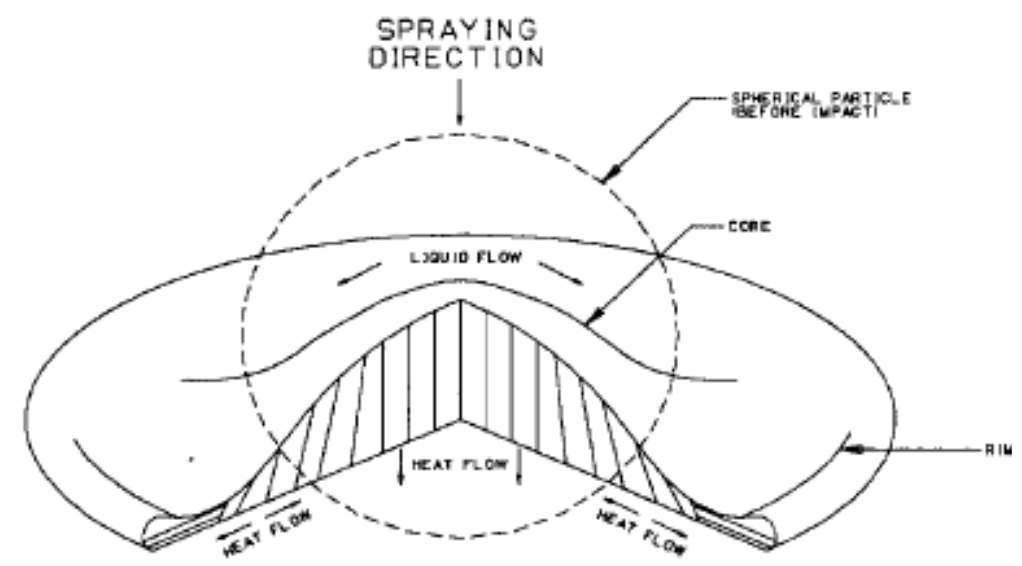

Figure 26: Plasma spray splat impact formation [75].

The degree of flattening is then given by the ratio between the splat and molten particle diameter. As is the porosity, this parameter depends on the velocity of the particle and plasma, as well as the density of the coating material and the kinetic viscosity of the melt point [76]. A typical flattening parameter of a 
deposited zirconia particle is around seven and upon solidification within the zirconia splat a columnar microstructure is formed, Figure 27 [77].

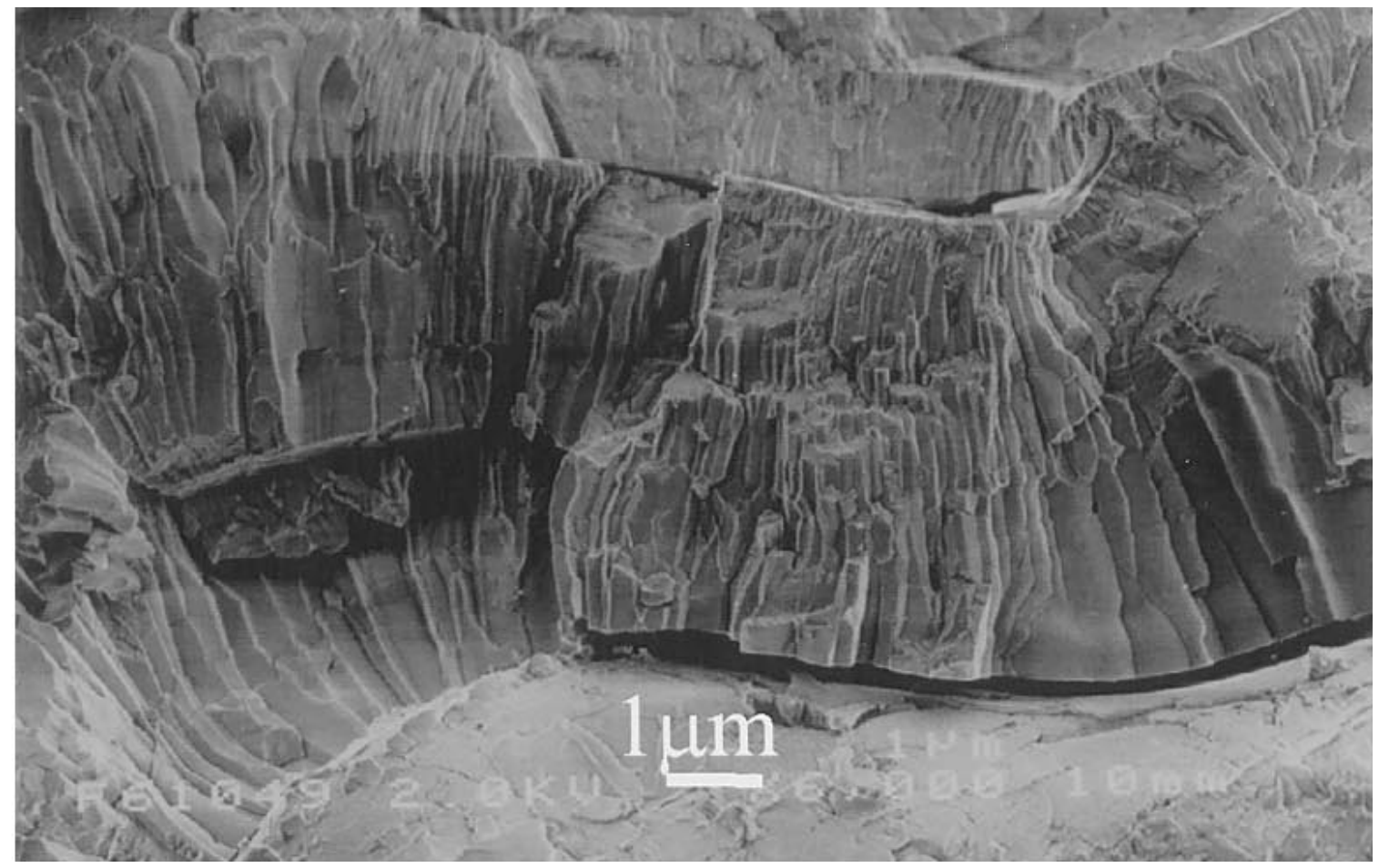

Figure 27: Plasma spray splat microstructure [77].

As a result of this quenching effect, deposited zirconia splats exhibit a large amount of residual stress. To accommodate this, stress reliving cracks develop within the brittle zirconia splats. Although cracks may form between splats, the microstructure within each splat however is well defined.

\subsection{Failure Mechanisms}

Although nearly all researches agree on the significance of bond coat oxidation for TBC life, the exact failure mechanisms for the various coatings types are not completely clarified. Complicating matters further, time to failure is also largely dependent upon cycle frequency and temperature, whereby considerable scatter is observed for even a single coating system [78]. In some cases failure is associated with the formation of brittle nickel and chromium rich spinel oxides [79]. Yet, these oxides only develop once the aluminum depletion zone reaches a critical value and are rarely seen as TBCs often fail prior to this. 
Generally, aluminum oxide growth is believed to be one of the major degradation mechanisms controlling the lifetime of TBC systems, yet no successful failure criteria using only oxidation rate has been developed. For more than a decade, the influence of bond coat oxidation behavior on TBC lifetime has been described simply by the thermally grown oxide (TGO) thickness [80, 81]. However, it has recently been shown clearly that the adherence of the YSZ/TGO/BC interface is the most important parameter governing the durability of the system, and therefore the lifetimes of these components are determined by the microstructural evolution taking place at these interfaces when exposed to thermal loads [68]. Generally, failure of in service EB-PVD TBC coatings is found to occur by spallation between the BC and TGO or solely within the TGO. Where as, APS TBC coatings are observed to failure at or near the thermally grown oxide, often propagating to and from the top coat. Due to the complex nature of these failure mechanisms, spallation observation of both EB-PVD and APS TBC coatings are detailed and described further in the following section.

\subsubsection{EB-PVD}

The most commonly observed and widely reported failure mechanism of EB-PVD TBC systems is crack initiation and propagation at the TGO/BC interface followed by a macroscopic buckling and spallation failure of the entire TBC system. This oxide scale delamination can be separated into two distinct failure mechanisms, buckling and wedging [82, 83, 84]. Buckling mechanisms are found to be the dominate mode of failure for compact scales with small concentrations of defects and relatively low interfacial toughness, while wedging is more likely to occur around thick scales with a large number of defects and strong oxide/metal interface. Yet, if macroscopic TBC failure is observed, buckling is likely responsible. Since the critical stress for buckling to occur depend on the ratio of TBC thickness versus delamination size, large scale delaminations are necessary. That is to say, the critical stage for EB-PVD TBC coating lifetime is crack growth at the oxide/metal interface, rather than a coalescence of crack initiation sites. The rate at which cracks propagate is highly 
dependent on interfacial toughness, yet the presence of water vapor in the test atmosphere accelerates this process [85]. Ultimately, the driving force behind both crack initiation and propagation is the elastic strain energy that accumulates in the TGO upon cooling and roughing or rumpling of this interface as a result of non-uniform oxide growth, Figure 30 [86].
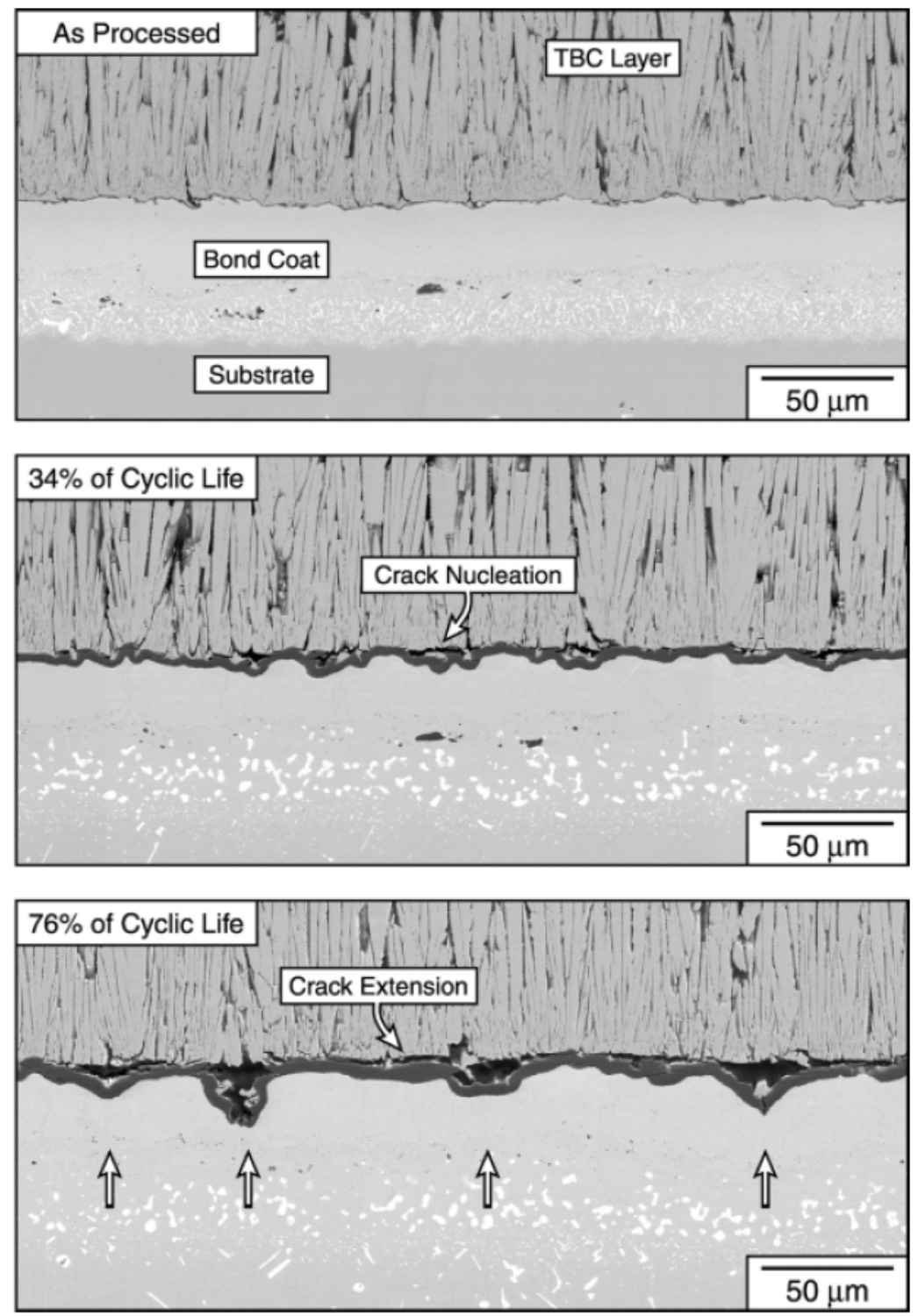

Figure 28: EB-PVD TBC cross-sectional scanning electron micrographs of Pt-aluminide bond coats showing the imperfections that enlarge by ratcheting. [86].

The large coefficient of thermal expansion mismatches that exist between the oxide scale (TGO) and both the BC and YSZ create large amounts of compressive residual stress within this layer; this however is not the direct cause 
of failure. Models aimed at using only critical scale thickness as the defining parameter for failure prediction have been largely unsuccessful, where in no

oxide scale thickness valid for all systems has been found accurate. It was observed that TGO layers having physical defects and increased amounts of reactive element oxides pegs (spinel) are capable of maintaining good adherence in spite of enhanced oxide growth rates when compared to that of thin, defect free scales $[87,88,89]$. Ultimately, these coatings are thought to be more durable than APS TBCs, yet the variation in application cost and faculties prevent this application procedure from becoming prevalent.

\subsubsection{APS}

As previously stated, the TBC failure mechanisms of greatest concern are those associated with the growth of an oxide scale between the YSZ and BC layers [90]. Eventual TBC spallation failure occurring at or near this layer has been continually observed by many [91]. Yet, microstructural analysis of isothermal and cyclically exposed TBC systems has also revealed a large amount of cracking occurring within the YSZ, TGO, BC and their interfaces [92]. These findings have led to the revelation that it is ultimately a coalescence of micro-cracking within the YSZ, TGO, BC and their interfaces that lead to large scale spallation failure of APS TBCs, where edge and buckle-driven delaminations compete as mechanisms for final spallation failure, Figure 29 [86]. 

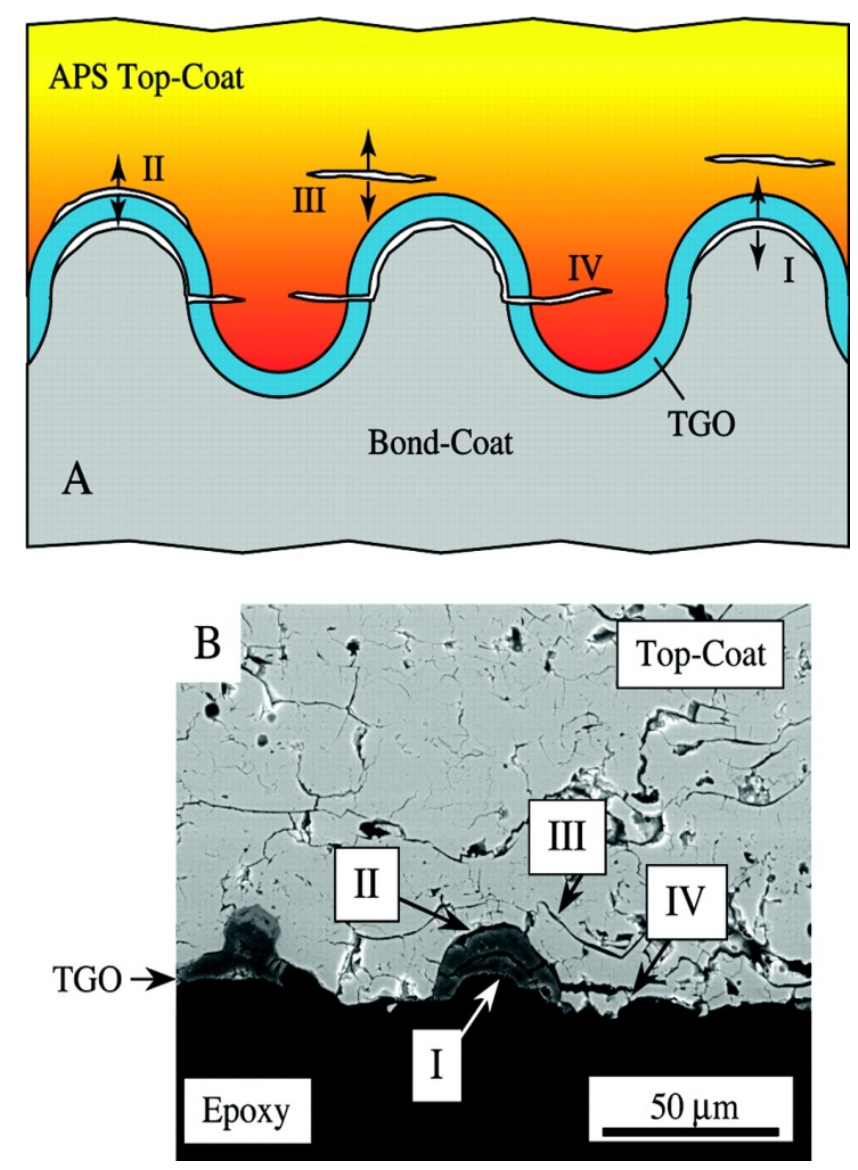

Figure 29: Schematic diagram and scanning electron micrograph showing the four different cracking mechanisms of a failed APS TBC after 240 cycles [93].

Once thought to provide additional adherence, the bond coats used in APS TBCs typically have high surface roughness or height asperity along their interfaces. Yet more recent analysis show that upon cooling, these non-uniform interfaces lead to the development of both in-plane and out-of-plane residual stresses, the degree of which is highly dependent on BC and YSZ coating application procedure, thickness and geometry [93, 94]. Furthermore, additional roughing or rumpling of this interface as a result of non-uniform oxide growth leads to the development of supplementary geometrical imperfections within the TGO further enhancing these residual stresses, Figure 30 [94]. 


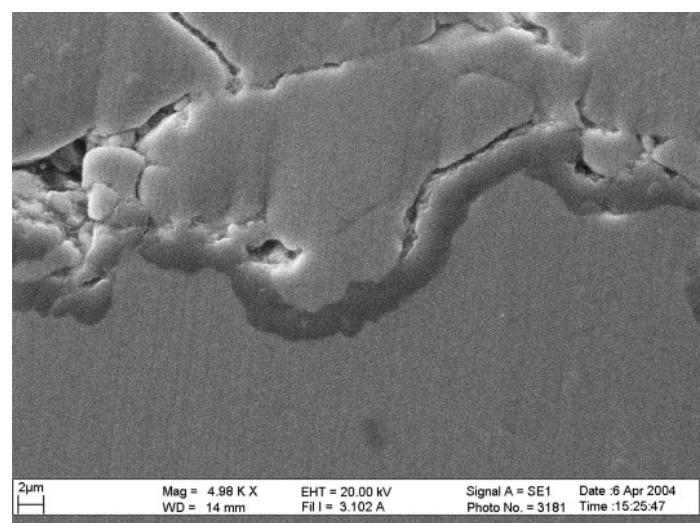

(a)

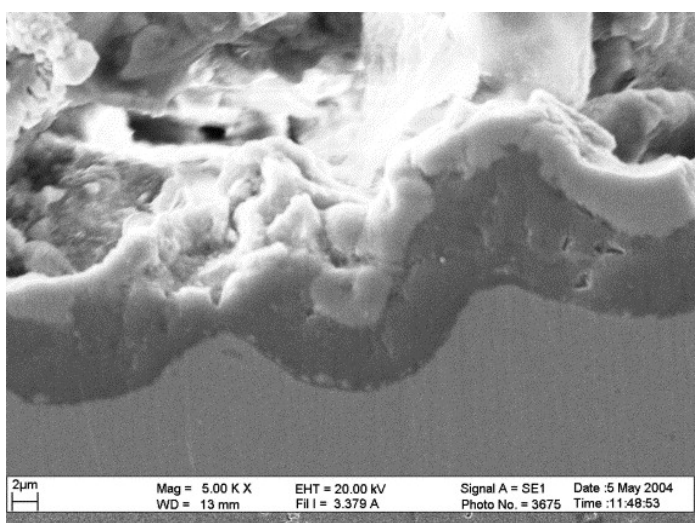

(b)

Figure 30: Cross-sectional scanning electron micrograph of an APS TBC system thermal load at $1070^{\circ} \mathrm{C}$ in air after a) 95 and b) 585 hours [94].

At these locations stresses become amplified and lead to the development of micro-cracking in and around the YSZ, TGO, BC and their interfaces [95, 96]. These initially developing micro cracks generally occur only at the peaks of the TGO/BC interface, yet do not propagate beyond this point, Figure 31 [97].

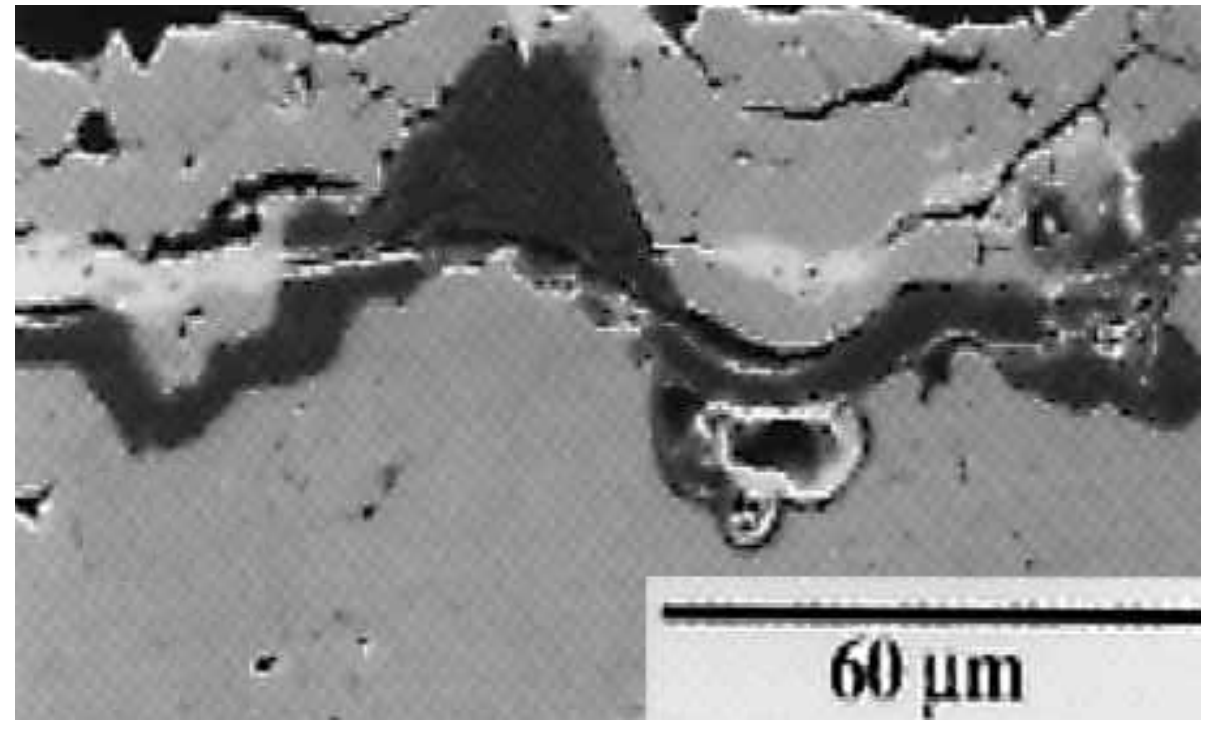

Figure 31: APS TBC system micro-crack development [97].

This suppression of propagation is generally attributed to the presence of compressive out-of-plane stresses that exists in the YSZ/TGO/BC interface troughs. Furthermore, the cause of this becomes more apparent when modeling stress distributions within an APS TBC system, where in models indicate that crack propagation within the TBC system in the YSZ/TGO/BC interface troughs 
require relatively high out-of-plane tensile stresses to initiate. Yet these stresses do not develop until a sufficiently thick TGO is formed. Additionally, numerical finite element models have also shown residual stresses within this area to be compressive rather than tensile when a thin, uniform TGO exist early in the TBC life cycle, Figure 32 [98].

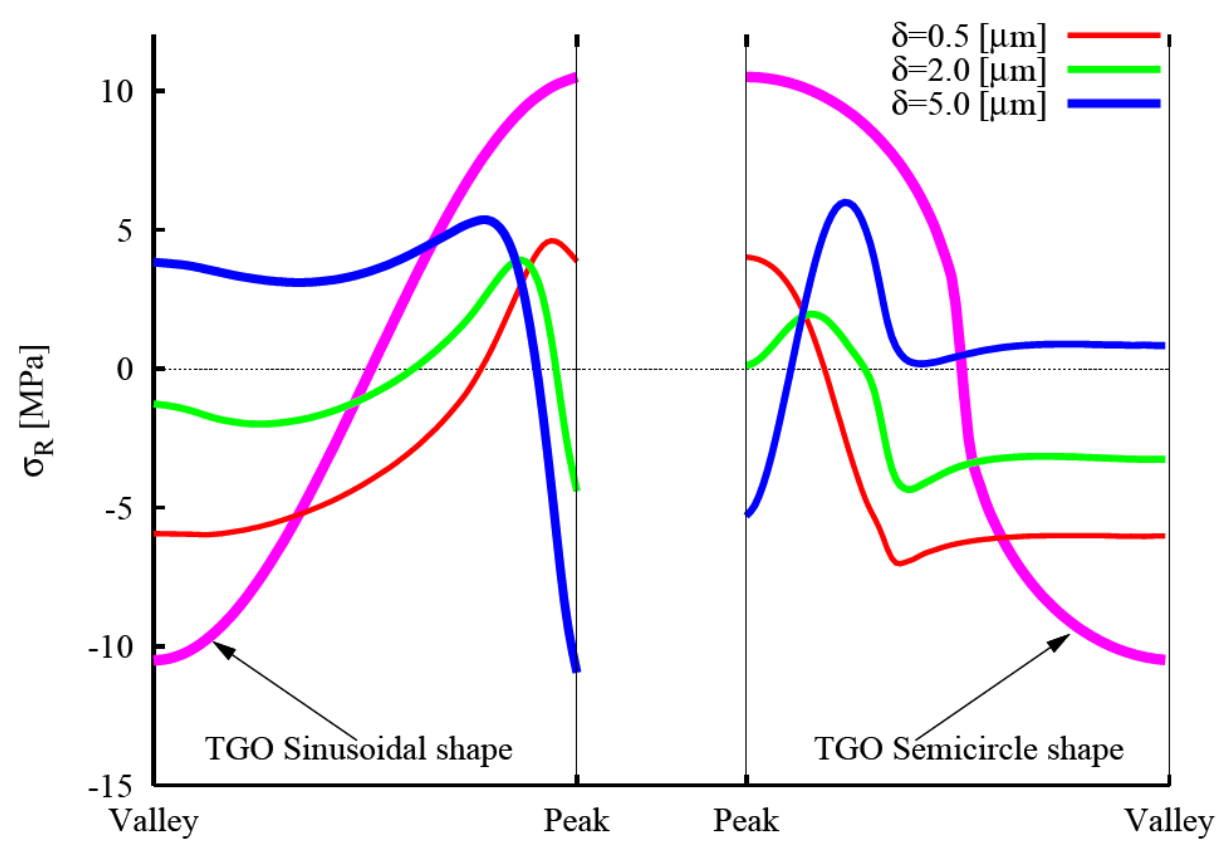

Figure 32: Oxide scale thickness induced stress analysis [98].

Although these delaminations at the YSZ/TGO/BC interface peaks occurs relatively early in the TBC's life cycle, these cracks are not completely responsible for macroscopic failure due to reasons previously mentioned as well as their relatively slow rate of prorogation. Experimental observations have shown the rate at which these TGO/BC cracks propagate to be much slower than the rate at which cracks within the YSZ prorogate. This mechanism typically leads to a TGO morphology consisting of repeated delaminations and cracking followed by re-growth, Figure 33 [99]. 


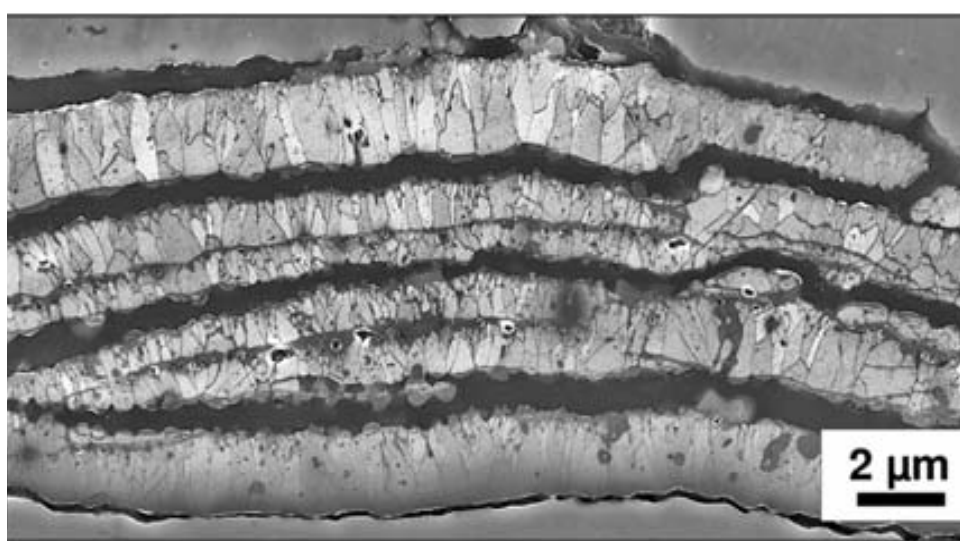

Figure 33: TGO fracturing and re-growth [99].

As the TGO/BC interface cracks develop, this now provides a location for expedited oxygen ingression and additional oxide scale growth. This process continues until a sufficiently thick TGO is formed to induce out-of-plane tensile stresses within the YSZ/TGO/BC interface troughs. As a result, APS TBC crack growth is considered to be a two phase mechanism, including both incubation (Phase I) and propagation (Phase II) phases [100, 101].

The first APS TBC failure phase, incubation, encompasses up to $50 \%$ of the systems life cycle. During this phase crack length is assumed to increase proportionally to TGO thickness and only occurs at the TGO/BC interface peaks. The slow crack propagation rate and out-of-plane compressive stresses with the YSZ/TGO interface do not allow these cracks to propagate beyond this region [102]. Yet, once a critical TGO height or crack length is reached this phase immediately ends and is followed by a second phase in which accelerated crack growth exist, Phase II. The remaining microscopic crack growth occurring throughout this propagation phase ultimately leads to large scale spallation failure. Due to this expedited crack growth, during this phase a fracture mechanics approach is utilized wherein stresses induced by the thermal expansion mismatch between the components of the TBC system, as well as those induced by TGO growth are taken into account [103]. It is in this second phase that the out-of-plane residual stresses within the YSZ/TGO interface troughs reach the critical level required to induce fracture within the YSZ and develop failure patterns containing both TGO and YSZ caps, Figure 34 [104]. 


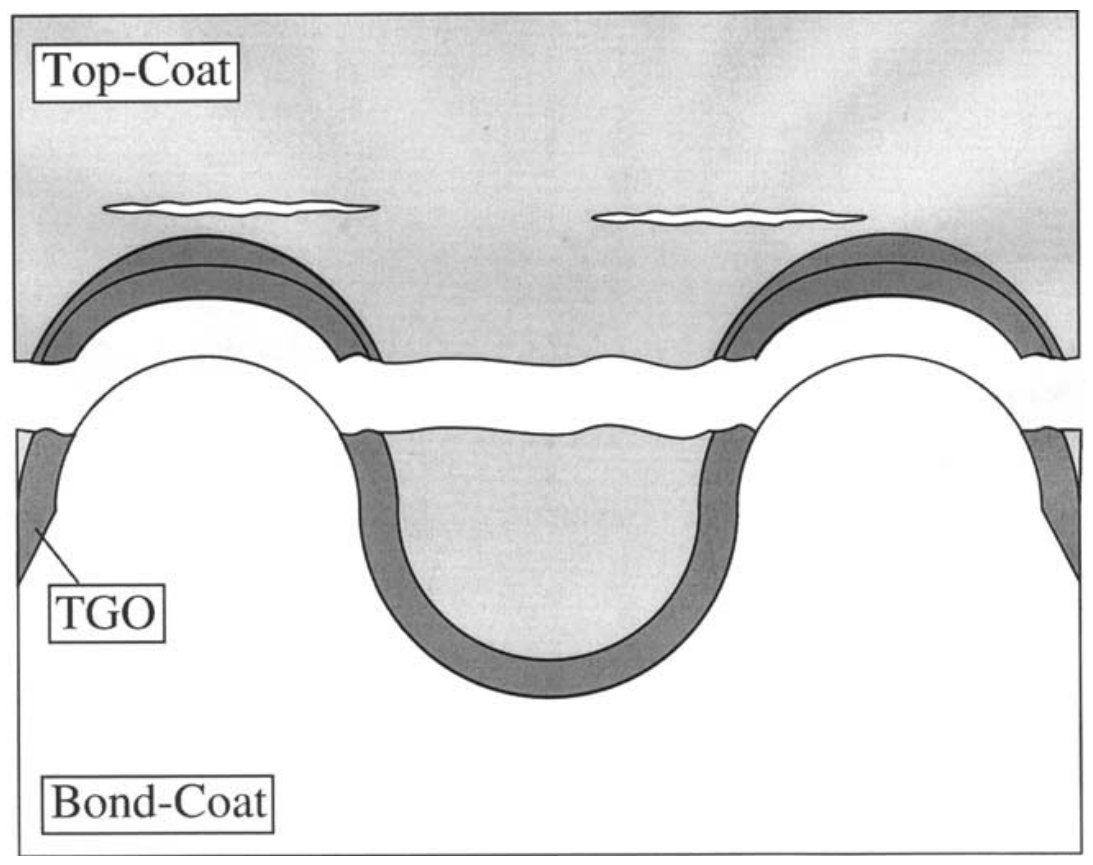

Figure 34: APS TBC failure pattern [104].

Ultimately, the strain energy accumulating in the TGO beneath the APS YSZ is the driving force for crack initiation and propagation resulting in macroscopic TBC failure. Researchers using $\mathrm{Cr}^{3+}$ photoluminescence piezospectroscopy (CPLPS) have shown TGO compressive residual stresses to be on the order of 1 3.2 GPa for APS TBCs [104]. Others utilizing Raman and X-Ray Diffraction $(X R D)$ techniques have measured stress values within the YSZ ranging anywhere from $92 \mathrm{MPa}$ at the surface to $660 \mathrm{MPa}$ at the YSZ/TGO interface, Figure $35[106,107]$.

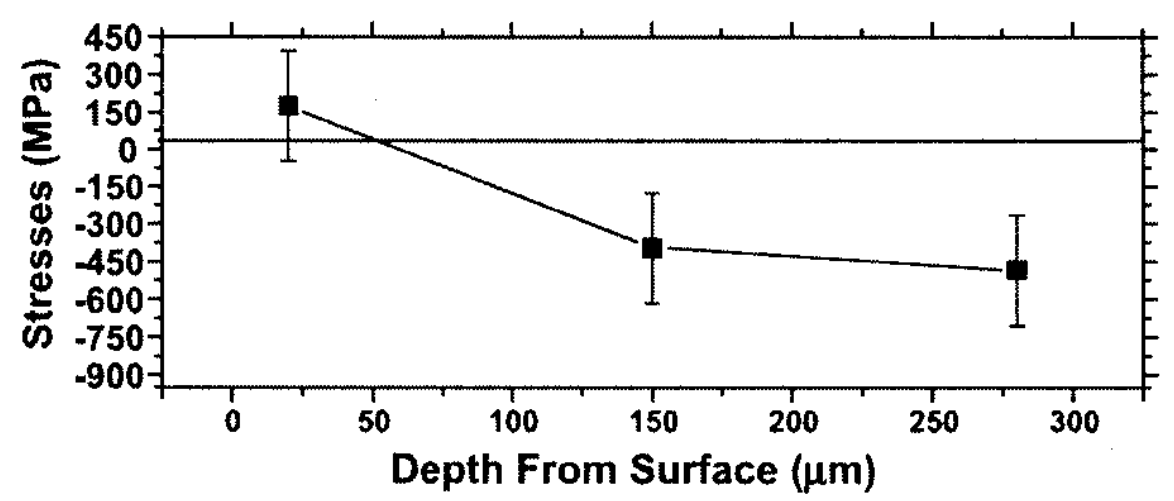

Figure 35: YSZ residual stresses measured by XRD techniques [106]. 
It has long been known that residual stresses in TBC systems create variations in behavior, performance and lifetime of the coated components [108], yet macroscopic failure is ultimately the result of a micro-crack coalescence progression. Although TGO growth is indirectly responsible for macroscopic TBC spallation failure, defining a critical TGO thickness upon which this occurs is unattainable.

\subsection{Non-Destructive TBC Evaluation}

The durability and life cycle of TBCs applied to gas turbine blades and combustor components is limiting the maximum temperature and subsequent efficiency at which these engines can operate. The development of new materials, coating technologies and evaluation techniques is required if enhanced efficiency is to be achieved. Current non-destructive TBC degradation inspection techniques and subsequent life cycles to failure predictions are made via visual inspection or by use of numerical estimation models, neither of which is highly accurate. Also missing from both techniques is a true measure of material degradation, this flaw results in the premature and often overdue replacement of combustor components. The ultimate result of such problems is additional cost, where blades are replaced prior to their life cycle fatigue limits baring catastrophic failure. Recent advancements in the non-destructive evaluation of TBCs include techniques that utilize acoustic emission, eddy current, microwave, emission spectroscopy and photo-acoustic technologies among others $[109,110,111,112,113,114]$. Although providing a true value of measure, these techniques are only capable of detecting spallation failure after its occurrence rendering them unreliable and just as time consuming as conventional methods. In the following section details of the more prevalent and successful techniques are presented.

\subsubsection{Infrared Imaging}

Local TBC spallation and delamination, when in the high temperature environment of an operational gas turbine introduces areas of low resistivity 
within the protective coating. This reduction in resistivity creates hot spots directly bellow spalled areas along the surface of the underlying superalloy substrate. Able to identify these regions, infrared sensors are sensitive to variation in temperature across an image. Siemens Power Generation, under sponsorship from the United States Department of Energy utilized this technology to develop a system for real time, on-line TBC coating failure protection and life maximization.

Using a sapphire lens as a window between the turbine and the external environment, high speed, high resolution infrared cameras are able to keep gas turbine blades in operational conditions under constant surveillance. Employing a blade and rotor synchronization system installed within the turbine, specific blade locations are continuously monitored for defects. The system is based on a telescopic arrangement consisting of a lens objective head and a series of relay lenses with the final relay acting as the system focus. A focal plane array infrared thermal wave imaging sensor is used to detect precursors to large scale TBC spallation failure [115]. The size and intensity of these precursors are used in an experimentally generated numerical model to determine time to failure or provide a repair request. Installed on a commercial gas turbine in late 2004 the system uses both near and mid wave infrared cameras to monitor blades in rows 1 and 2 . Although the system is non evasive and thus completely non-destructive, this technique detects TBC spallation and delamination after its occurrence only. Prior to spallation a considerable reduction in TBC overall continuity has occurred allowing the ingress of high temperature gasses further reducing the structural integrity of the superalloy substrate. Also, the system has a minimum life span of 8,000 hours where as many power generating gas turbine engines remain operational for much longer.

Additional research conducted in the field of mid-infrared reflectance imaging has shown promise of becoming a reliable diagnostic tool for monitoring delamination progression in TBC systems as well, [116, 117, 118, 119]. 


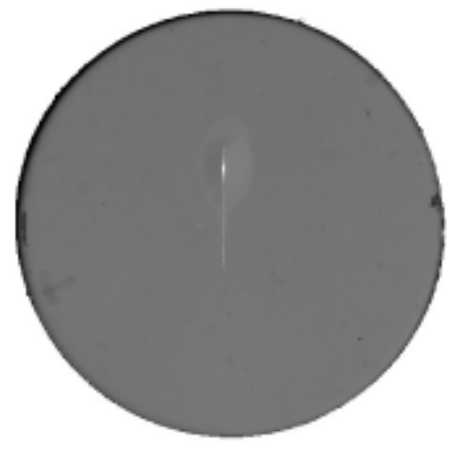

(a)

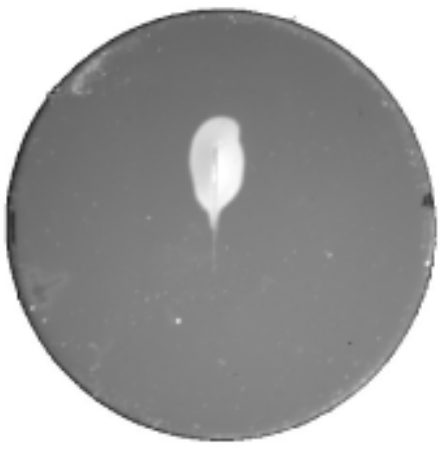

(b)

Figure 36: Delamination indication by a) white light and b) mid-infrared imaging [116].

Mid-infrared reflectance imaging utilizes the maximum transparency of TBC systems in the 3 6 $4 \mathrm{~m}$ wavelength to evaluate sub-surface delamination crack propagation that is typically hidden from visible wavelength inspection. The image contrast that identifies delamination progression arises from the increased reflectance produced by a large component of total internal reflection at the crack interface. The short wave lengths used allow this technique to detect delamination progression at the early stages. However, this technique as is the traditional infrared imaging, extremely costly.

\subsubsection{Photoluminescence Piezospectroscopy}

In this technique the integrity of TBC system interfaces is examined using $\mathrm{Cr}^{3+}$ photo-stimulated luminescence piezo-spectroscopy (CPLPS). Developed initially for the determination of residual stresses in polycrystalline alumina, luminescence has also been applied to residual stress measurement within developing oxide scales and overall interface degradation within TBCs [120, 121]. The basis for the measurement is the piezo-spectroscopic shift in the R-line fluorescence from $\mathrm{Cr}^{3+}$ impurities incorporated into the growing aluminum oxide scale. The observed piezo-spectroscopic shifts are attributed to residual stresses in the TGO. Although YSZ coatings are relatively transparent at both the excitation and fluorescence frequencies, the signal is not always strong and without noise. 
Later refinement of this technique has shown an enhanced ability to observe residual stresses and polymorphic transformation within the aluminum oxide that makes up the TGO $[122,123]$ for both APS and EB-PVD TBCs. Further investigation found that for strained $\alpha-\mathrm{Al}_{2} \mathrm{O}_{3}$, the $\mathrm{R}_{1}$ and $\mathrm{R}_{2}$ doublet fluorescence undergo a systematic shift in frequency when stress is applied. From this, a measured coefficient of piezo-spectroscopic tenors can be identified. Using this coefficient, the stress within the $\alpha-\mathrm{Al}_{2} \mathrm{O}_{3}$ can be identified assuming the thin $\alpha-\mathrm{Al}_{2} \mathrm{O}_{3} \mathrm{TGO}$ is in a bi-axial stress state and has randomly oriented polycrystalline grains [124]. More recent work has established that in addition to stress measurements, both the standard deviation and bimodal fraction of the luminescence signal show systematic changes with thermal exposure providing a more complete and accurate coating degradation assessment when using this technique [125].

Combining the research efforts of those using luminescence and infrared technology this method, unlike those previously discussed, has subsurface selectivity with the TBC/TGO interface. By employing a laser wavelength to which the YSZ is transparent but the TGO absorbs, a stronger and clearer signal is acquired. In this technique, the energy provided by the laser is converted into both luminescence and heat within the TGO. Using a mid-infrared detection sensor, the subsequent change in temperature of the TGO is measured as heat is conducted away from the TGO into the adjacent alloy and YSZ. Damage of the TBC system decreases the rate at which the heat dissipates and can be quantified from measurements of transient temperature change. The remaining luminescence signal is then again used to provide a residual compressive stress measurement within the TGO as well as a total thickness [126]. Although successful in a controlled lab environment, no large scale system utilizing this technology has been developed for use with land based gas turbines. Also, stress measurements alone have proven unable to accurately predict failure of thermal barrier coatings. 


\subsubsection{Electrochemical Impedance Spectroscopy}

Electrochemical impedance spectroscopy has long been used as a nondestructive evaluation technique [127]. Of late much research has been aimed at using this technology as a non-destructive technique for TBC degradation evaluation. The principle of this technique is to relate electrical properties of the material to its microstructural features [128]. Defects such as delamination, spallation and cracking with the TGO can be detected by evaluating the voltage and current responses of the TBC using alternating current with varying frequency. Variations in both resistance and capacitance of the TBC system are found to be associated with the growth of alumina scales and overall TGO degradation [129]. Along with degradation evaluation, impendence spectroscopy is able to evaluate YSZ and TGO thickness as well. Furthermore, with an overall TGO thickness measurement, a differentiation between $\theta-\mathrm{Al}_{2} \mathrm{O}_{3}$ and $\alpha-\mathrm{Al}_{2} \mathrm{O}_{3}$ I n this layer can be defined. Impedance analyses demonstrate that the resistance of the alumina scale increases and the capacitance decreases with thermal exposure [130]. However, a gradual decrease in the YSZ resistance was observed over prolonged thermal exposure.

During impedance measurement, an electrode is applied to the top surface of the TBC with the nickel based super alloy substrate acting as the other electrode. An electric field with varying frequency is then applied and directed through the TBC system. Electrical responses from the various components of the TBC system (YSZ, TGO and BC) reflect in differing frequency domains [131]. Due to this, the electrical response from the multiple layers can be separated in the impedance spectra and thus the microstructure of the TBC can be correlated to these impedance spectroscopy parameters. Variations in the TBC microstructure along thermal exposure lead to changes in electrical material properties and thereby variations in impedance spectra.

Highly temperature dependent, this electrical signal is highly affected by temperature, moisture and dopants in the material. Furthermore, YSZ'S low electrical conductivity makes this measurement quite difficult. As electrode size effects the measurement as well, a small electrode is used yet this increases 
evaluation time. It is also desirable to minimize the effects of surface leakage current but difficult. Additionally, testing response is highly dependent on location due to localized TGO and spinel deposits; this may lead to premature or delayed replacement of hot section gas turbine components. Moreover, this system is primarily obtains response from the alumina oxide layer forming within the TBC and not the overall degradation state.

\subsubsection{Acoustic Emission}

Acoustic emission ( $\mathrm{AE}$ ) techniques have long been used in studies to investigate the performance and failure behavior of plasma-sprayed thermal barrier coatings [132]. As degradation cracking of the TBC initiates, internal stresses acting within this system redistribute releasing acoustic emission waves that using a piezoelectric sensor can be monitored. TBC samples monitored by $A E$ techniques throughout thermal cycling under infrared quartz heat lamps have shown that sources of high AE activity develop cracks [133]. Furthermore, this technique can discriminate between vertical cracks at the surface and delaminations of the interface by analysis of the AE signals emitted during the heating and the cooling periods of thermal cycling. This technique however does not provide earl detection of TBC cracking, only notification that it has occurred.

\subsubsection{Interferometric Techniques}

All interferometric techniques, this includes holographic, thermal wave and electron speckle pattern, operate on the basic principle of interference pattern information about the object being analyzed. Thermal wave interferometry has been extensively developed for the non-destructive evaluation of plasma-sprayed coatings, wherein addition to detecting the presence of sub-surface defects, it has also been shown to be capable of determining the thickness and thermal properties of such coatings [134]. These techniques are attractive because they are non-destructive and non-contacting, furthermore, they can provide information about certain coatings which cannot be readily tested with prevailing evaluation techniques [135]. Yet as do the other prevailing non-destructive 
evaluation techniques, interferometric technologies are only capable of detecting failure after its occurrence. Thus no predetermination of thermal cycles to failure can be reported.

\subsection{Load Based Micro-Indentation Evaluation}

The objective of the following research is to develop a non-destructive TBC degradation and spallation evaluation technique by use of a spherical instrumented indentation technique. This method, as does any non-destructive technique (NDE), must first be proven truly non-destructive. Regardless the successfulness of the practice, if damage is in induced upon evaluation the technique is deemed impractical. Although destructive testing practices have been utilized in many applications, due to the cost of both the blade as well as the coating application procedure, TBC coated hot section turbine components are rarely evaluated in this manner. To validate the non-destructive nature of this micro-indentation technique several coupons were subjected to both thermal loadings and degradation evaluation while others were only exposed to thermal loadings. Ultimately, both samples were found fail at comparable cumulative thermal hours. Furthermore, indented and non-indented areas were analyzed via scanning electron microscopy (SEM) for additional cracking in or around the evaluated regions. This comparison revealed no additional degradation between the indented and non-indented regions.

Additional details and images confirming this approach are presented in a later section. Also required for any NDE technique, this method must be proven repeatable across multiple conditions and materials. Due to the complex nature of the multilayered TBC system, although chemical composition may be uniform the microstructure following application is not. Furthermore, as the thermal barrier is exposed to elevated temperatures, oxidation, cracking and sintering effects increase microstructural and chemical variability across even a single coating. This unique feature of the TBC system further complicates matters of developing a truly non-destructive spallation prediction evaluation technique. To demonstrate the consistency of this indentation based failure prediction 
methodology multiple samples cycled at various conditions are evaluated. Moreover, TBC systems applied by both APS and EB-PVD processes have been thermally cycled as well. The results of this study show that using a multiple partial unloading procedure, failure locations for both isothermal and cyclically exposed APS/MCrAlY/RenéN5 as well as EB-PVD/MDL/RenéN5 TBC coupons were correctly predicted with a $90 \pm 3 \mathrm{~N}$ evaluation load.

TBC systems were subjected to various thermal cyclic loading conditions in an ambient air environment at $1100^{\circ} \mathrm{C}$. Following heat treatment all coupons are evaluated using a load based multiple partial unloading micro-indentation procedure. A displacement controlled algorithm was implemented in which a total actuator displacement of forty microns was used encompassing twenty multiple partial unloading/loading of one and two microns respectively. The indentation instrument used throughout this system was custom built and used only for evaluation of TBC materials. Furthermore, using LABVIEW'T control software designed to perform only multiple partial unloading tests and any subsequent data reduction is utilized. Through this technique both the load and slope at each unloading could be evaluated during the study. All evaluations of TBC coupons were performed at room temperature on a vibration damping optical table. Additional details of the thermal cycling conditions and evaluation tests are presented bellow.

\subsubsection{Thermal Barrier Coating Coupons}

The TBC coupons used in this study consisted of a 1/8" thick by 1" square nickel base single crystal superalloy RenéN5 substrate followed by a $300 \mu \mathrm{m}$ MCrAlY bond coat layer and subsequent $350 \mu \mathrm{m}$ seven weight percent yttria stabilized zirconia layer applied by an APS or EB-PVD process. Seven APS coupons were evaluated, three isothermally and three cyclically exposed to temperatures of $1100^{\circ} \mathrm{C}$ in ambient air environments. Where as only one EBPVD coupon was evaluated throughout this study and was cyclically exposed. Following high temperature exposure the samples are weighed, imaged, and evaluated using the aforementioned micro instrumented indentation technique for 
TBC degradation. A multiple partial unloading indentation procedure was utilized throughout this study, capable of allowing unloading slopes at multiple loads to be analyzed. A spherical tungsten carbide (WC) indenter having a radius of $793.5 \mu \mathrm{m}$, combined with a piezoelectric actuator $(3.6 \mathrm{~nm}$ Resolution, Physik Instrumente, P-239.9S, $180 \mu \mathrm{m})$ and a high accuracy $( \pm 0.15 \%$ Accuracy, Honeywell, Model 31, $100 \mathrm{lb}$.) load cell completed the indentation chain, Figure 37.

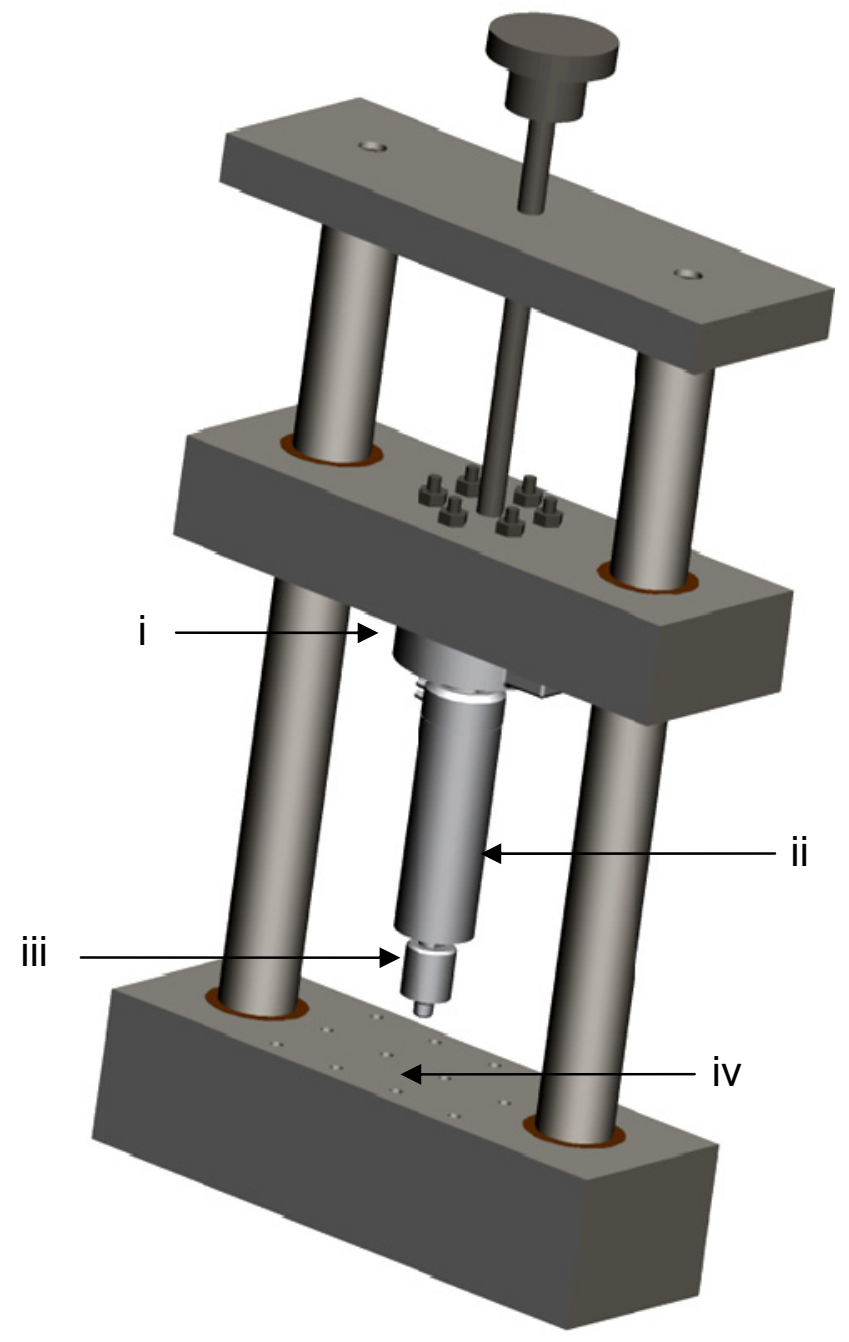

Figure 37: Experimental setup, i) $\pm 0.15 \%$ accuracy Honeywell Model $31100 \mathrm{lb}$. load cell, ii) $3.6 \mathrm{~nm}$ resolution Physik Instrumente P-239.9S $180 \mu \mathrm{m}$ piezoelectric actuator, iii) spherical tungsten carbide (WC) $750 \mu \mathrm{m}$ radius indenter, and iv) coupon stage.

The large radius indenter and high precision actuator minimized coating damage during unloading surface stiffness response evaluation. All indentation 
tests were performed on the same system enabling maximum consistency over the course of the study. Furthermore, the TBC coupons for these experiments were provided by the National Energy Technology Laboratory (NETL), a division of the United States Department of Energy (DOE).

\subsubsection{Thermal Exposure}

Isothermal high temperature exposures were performed in 40 hour increments wherein the coupon was brought to a temperature of $1100^{\circ} \mathrm{C}$ within 15 minutes followed by a 40 hour dwell period after which the coupon was removed from the furnace within 15 minutes as well. Coupon $A$ following a similar thermal ramping rate as did the other isothermally exposed TBC coupons was brought to temperature within 15 minutes, yet was not exposed in 40 hours increments but rather various dwell times $(10,15,20,50$, etc.). Although Coupon A did not receive similar heat treatment to Coupons $B, C$ and $D$, this variation in dwell period is far greater than that to which the cyclically exposed coupons experience. Thus Coupon A is characterized as an isothermally exposed TBC sample. Opposing that of the isothermally exposed coupons, cyclic thermal exposures encompassed a high temperature dwell period of only 45 minutes $(E$, $F, G$, and $H$ ). Yet, ramping procedures for each cyclic exposure consisted of the coupon being brought to $1100^{\circ} \mathrm{C}$ within 15 minutes removed from the furnace within 15 minutes as did the isothermally exposed samples. These cyclically treated coupons were exposed to 40 cycles per heat treatment. Throughout these cycles the samples were exposed to, but may have never reached room temperature conditions $\left(24^{\circ} \mathrm{C}\right)$. Both the cyclic and isothermal heat treatments were thermally exposed in a horizontal tube furnace able to maintain a set temperature of $\pm 3^{\circ} \mathrm{C}$ along with a programmable linear actuator on which the coupons were placed and inserted in the furnace. All thermal heat treatments are performed in ambient air environments. 


\subsubsection{Non-Destructive Verification}

Demonstrating the non-destructive nature of this micro-indentation testing technique when using a large radius spherical $(793.5 \mu \mathrm{m})$ indenter with relatively low maximum applied loads $(\sim 160 \mathrm{~N})$, an APS/MCrAIY/RenéN5 coupon was subjected to high temperature thermal exposure as well as micro-indentation testing and a second coupon was subjected to only high temperature thermal exposure. Observed to fail at comparable cumulative thermal cycles, the microindentation was found to have no significant effect on ultimate TBC system life cycles to failure. Also, YSZ plan section SEM analysis of the indented regions displayed no indentation induced cracking in or around the evaluated areas when compared to that of the non-indented coupon for both APS (Figure 38) and EBPVD (Figure 39) TBC systems.

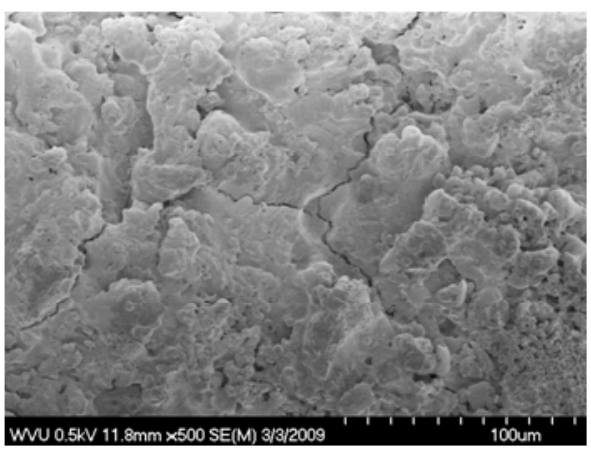

(a)

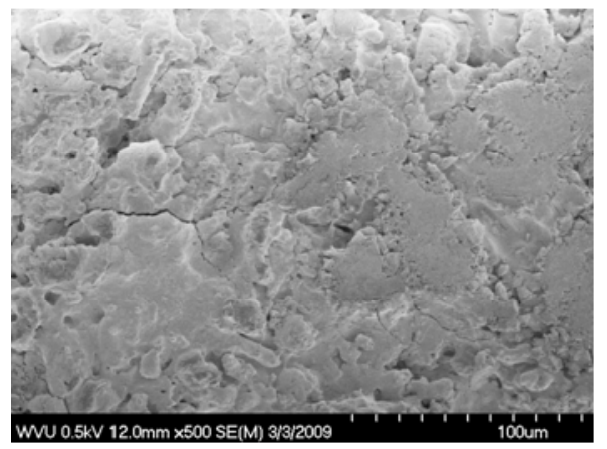

(b)

Figure 38: Top coat SEM micrographs of an APS/MCrAIY/RenéN5 obtained after a cumulative $\mathbf{3 7 5}$ isothermal hours, a) Non-Indented Region and b) Indented Region.

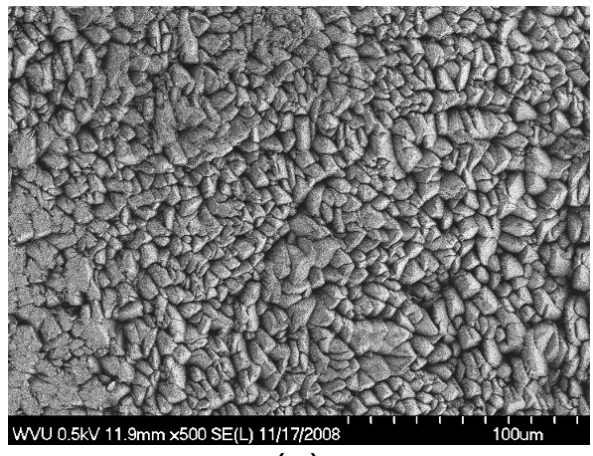

(a)

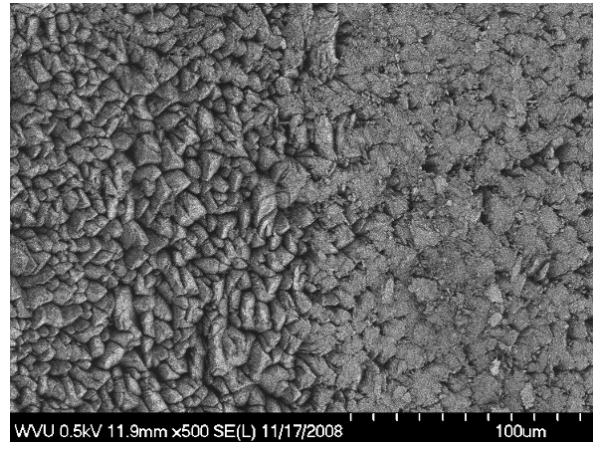

(b)

Figure 39: Top coat SEM micrographs of an EB-PVD/MDL/RenéN5 obtained after a cumulative 60 cycles, a) Non-Indented Region and b) Indented Region. 
Finally, optical micrographs obtained from an EB-PVD/MDL/RenéN5 coupon surface following failure clearly illustrate crack propagation paths avoiding both the center and edges of the evaluated areas, Figure 40. Through this process it was determined that the proposed micro-indentation degradation evaluation technique does not promote TBC system micro-cracking or debonding [136].
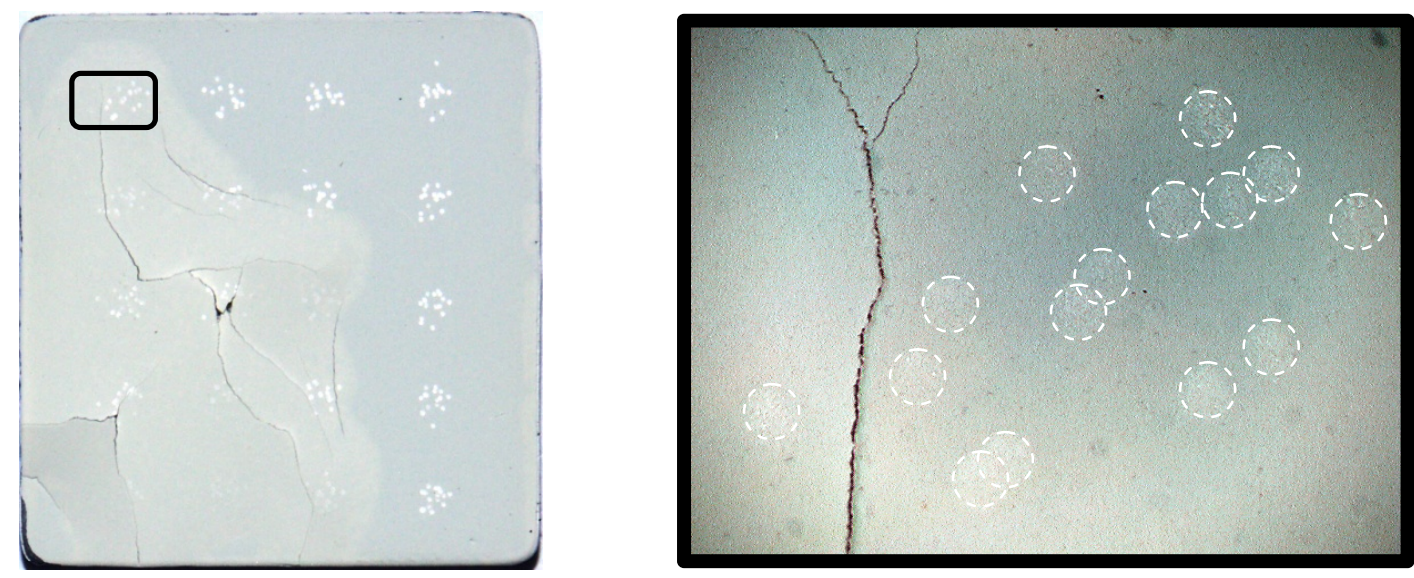

Figure 40: Failure location along the surface of the thermally cycled EB-PVD/MDL/RenéN5 coupon.

Additionally, as the TBC system is neither isotropic nor homogenous, indentation surface stiffness response and inference zone become functions of load and indenter radius. To investigate these effects, an axisymmetric TBC model has been generated to establish the level of inference from each TBC component, Figure 41. Having a width of $2000 \mu \mathrm{m}$ and a total height of $3562 \mu \mathrm{m}$ (TBC: $353 \mu \mathrm{m}$, BC: $209 \mu \mathrm{m}$ and Substrate: $3000 \mu \mathrm{m}$ ), the material properties imposed for each layer are found in Table 2 [104].

Table 2: Indentation inference zone TBC system material properties [104].

\begin{tabular}{|c|c|c|c|}
\hline Material Property & YSZ & BC & Substrate \\
\hline Elastic Modulus (GPa) & 50 & 211 & 150 \\
\hline Poisson's Ratio & 0.1 & 0.3 & 0.25 \\
\hline Yield Stress (MPa) & 250 & 422 & 841 \\
\hline Tangent Modulus (GPa) & 1 & 0.889 & 1.05 \\
\hline
\end{tabular}


Results of this simulation show the indentation induced stress zone (when a $90 \mathrm{~N}$ load is applied) extends through all layers when a $793.5 \mu \mathrm{m}$ radius indenter is implemented, Figure 42. This stress distribution indicates that the surface stiffness response measurement at $90 \mathrm{~N}$ on the TBC system is capable of acquiring some level of response (degradation, residual stress state, etc.) from all layers and their interfaces.

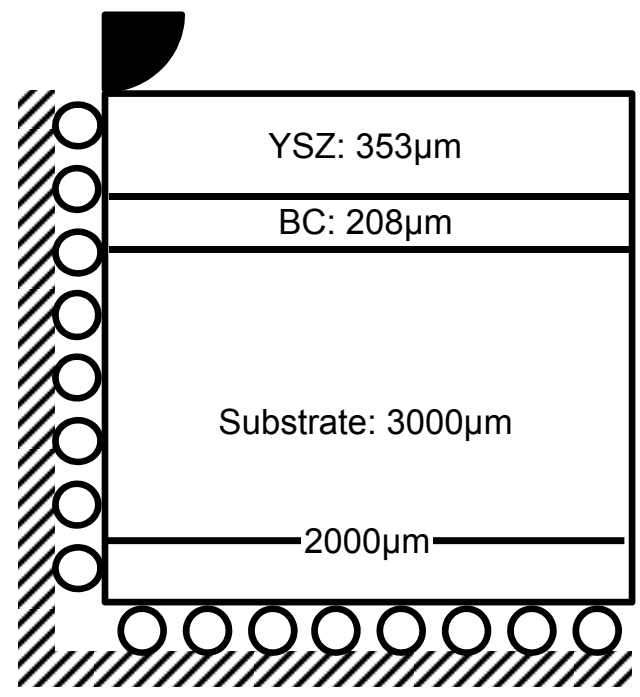

Figure 41: Axisymmetric numerical TBC indentation model boundary conditions and indenter radius of $793.5 \mu \mathrm{m}$.

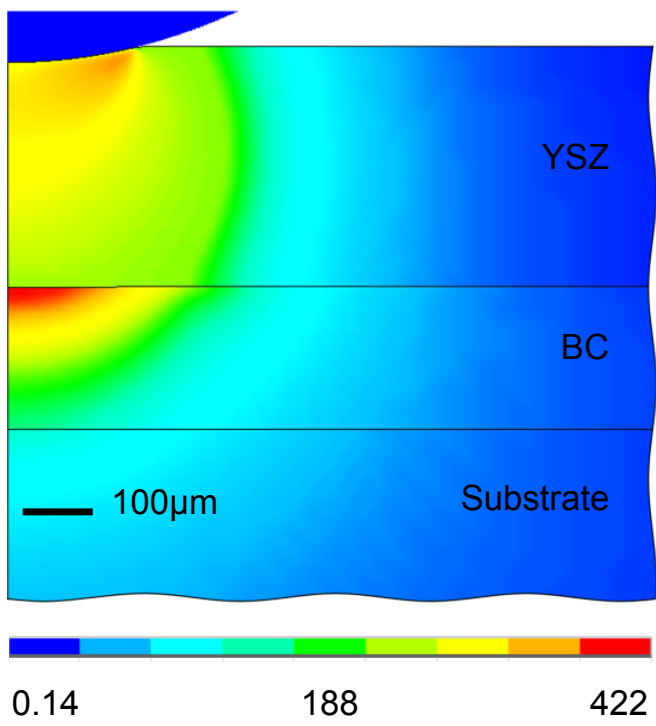

Figure 42: $90 \mathrm{~N}$ indentation inference zone shown to exist in all layers of the TBC system, Von Mises Stress (MPa).

\subsubsection{Unloading Slope Measurement Results}

Data presented in this section was obtained using the previously mentioned multiple partial unloading procedure, this technique allows for unloading slope stiffness responses at various loads to be monitored effectively. Performed on each coupon's surface were 20 evenly spaced micro-indentations $4 \mathrm{~mm}$ from the sample's edges, Figure 43. Unloading slopes obtained on each TBC coupon are presented using a contour color mapping scheme. The procedure used to generate each color map is as follows: a) acquire unloading slopes at a given load for each location upon which indentation was performed, b) assign each location a value according to the average and standard deviations obtained on the entire coupon, c) employ the use of a finite element interpolation 
algorithm to generate each contour color map using these values. Although slope data is obtained at multiple loads $(20 \sim 160 \mathrm{~N})$, the stiffness responses recorded at $90 \pm 3 \mathrm{~N}$ were shown to have manufactured the clearest degradation trends and were ultimately enlisted to predict spallation failure location.

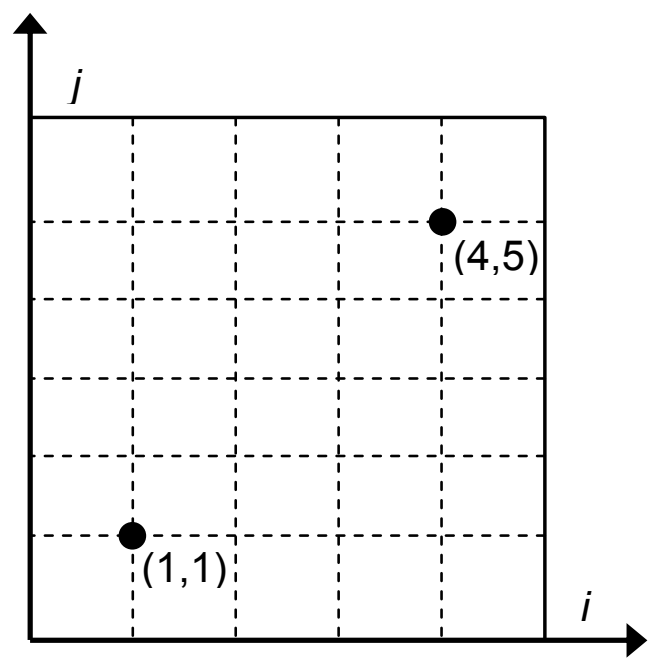

Figure 43: Indentation distribution.

As previously mentioned data was reduced through an evaluation of each locations value with respect to that of the total data sets average and standard deviation. Each data set contains only those values obtained from the coupon between each thermal exposure. Due to degradation of the TBC as consequence of thermal loading, results gathered from the coupon before thermal loading cannot be compared to those obtained after additional thermal loads are applied. Microstructural and coating interface changes over the life of the coupon restricts data comparison to only those within a given data set. An algorithm based on these principles produces a value of relative deference within the overall test set and is described as follows.

To begin the data reduction process, standard deviation of each data set is first calculated, Equation (11), where $y$ represents the unloading slope obtained at $90 \pm 3 \mathrm{~N}$ and $k$ the number of thermal loadings of which the coupon has been exposed [137]. Variables $i, n, j$ and $m$ are defined as the $\mathrm{x}$-axis test location, total number of test locations in the $x$-axis, $y$-axis test location and the total number of test locations in the y-axis respectively. As twenty tests are 
performed on each coupon per thermal cycle $n$ and $m$ are equal to 4 and 5 respectively. Also, $N$ is defined as the total number of tests per data set, thus this is equal to 20 .

$$
\sigma_{k}=\sqrt{\frac{\sum_{i, j=1}^{n, m}\left(y_{i j, k}-\bar{y}_{k}\right)^{2}}{N-1}}
$$

Defined as an outlier in this statistical analysis, any unloading slope greater than $80 \%$ of the first standard deviation is identified via Equation (12). Citing the empirical rule and assuming a normally distributed set, one standard deviation contains approximately $68 \%$ of all the data points. Defining the critically high sloped values as those within the top $20 \%$ of the first standard deviation and above, outliers encompass on average only $22.8 \%$ of all data points within the set, Figure 44 [138].

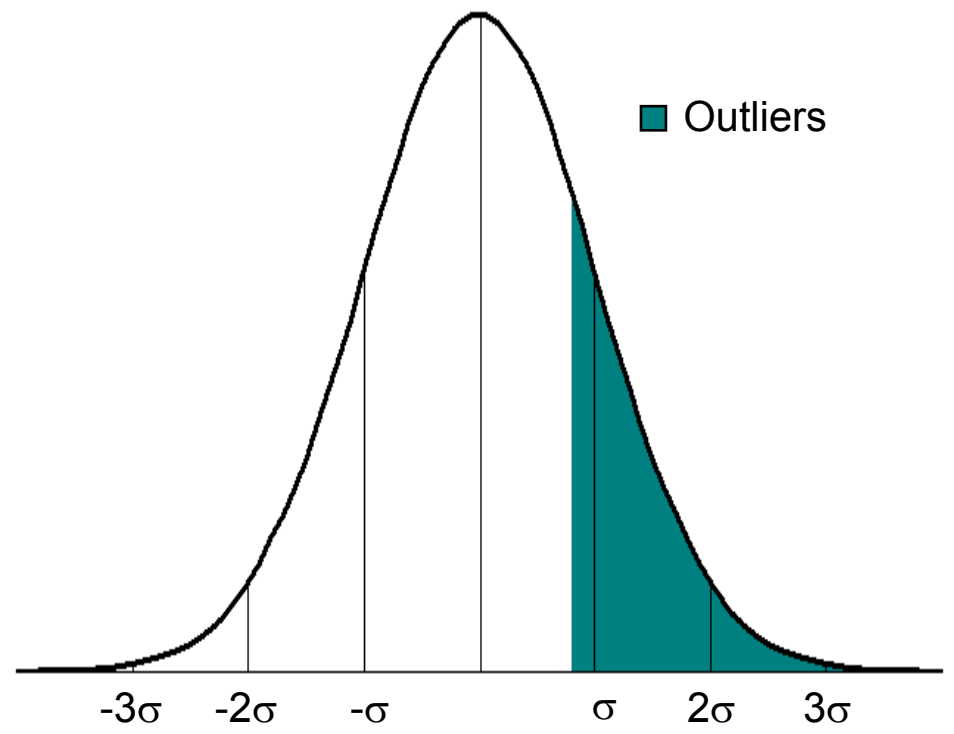

Figure 44: Outliers unloading slope measurements defined as those data points that reside within and above $\mathbf{8 0} \%$ of the first standard deviation.

These data points are considered to have higher residual stresses and thus are more prone to spallation failure. The remaining data points, those in the lower $77.2 \%$ and not critical and are assigned a value of zero, Equation (13).

$$
z_{i j, k}=y_{i j, k}-\left[\bar{y}_{k}+(0.8) \sigma_{k}\right]
$$




$$
x_{i j, k}=\left[\begin{array}{ccc}
0 & \text { if } & z_{i j, k} \leq 0 \\
z_{i j, k} & \text { if } & z_{i j, k}>0
\end{array}\right]
$$

Finally, each test locations relative level of spallation probability is found via Equation (14). As additional thermal loads are applied, the unloading slopes obtained at each location are added to those found during previous microindentation evaluations for prior thermal cycles. Through this process the contour color map for a coupon that has under gone I thermal exposures will display the summation of unloading slopes obtained in each location from thermal exposure zero to $l$.

$$
t_{i j}=\sum_{k=1}^{l} x_{i j, k}
$$

In the following sections, both images and contour color maps utilizing this procedure and data will be presented for both isothermally and cyclically exposed APS and EB-PVD TBC coupons. Unloading slopes obtained from each TBC coupon are presented using a contour color mapping scheme, examples of which are presented in later sections. The contour color maps provide a visual aid from which TBC spallation failure locations are predicted. Coupon failure for this study was characterized as visual edge delamination on the order of $1 \mathrm{~mm}$ unless otherwise stated.

\subsubsection{Isothermal Exposure}

Coupons $A, B, C$, and $D$ were isothermally exposed to ambient air high temperature environments for a cumulative total of 400 or 450 hours at $1100^{\circ} \mathrm{C}$. The coupons, three of which failed after 400 isothermal hours (coupons B, C and D) and one failing after 450 isothermal hours (coupon A) did not fail in unison. As previously stated coupon A was periodically removed from the furnace after 85 , $185,300,400$ and 450 cumulative hours of thermal exposure. Following a cumulative total of 400 isothermal loading hours, a visible corner spallation between sides 1 and 2 was found. After subjecting the coupon to two additional 50 hour thermal loadings and two weeks of storage, spallation along edge 1 was observed, Figure 45. 


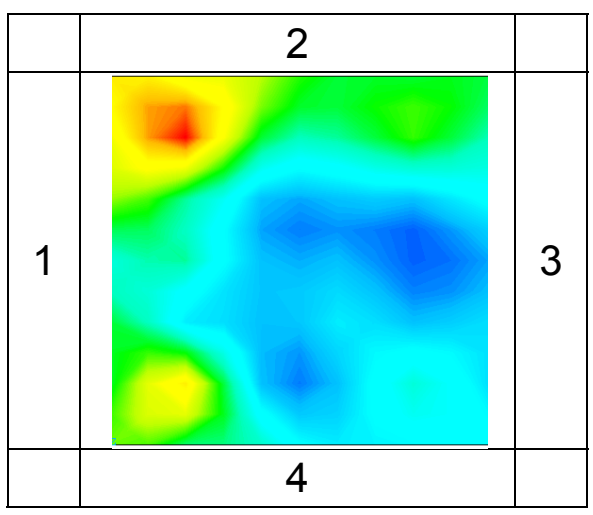

(a)

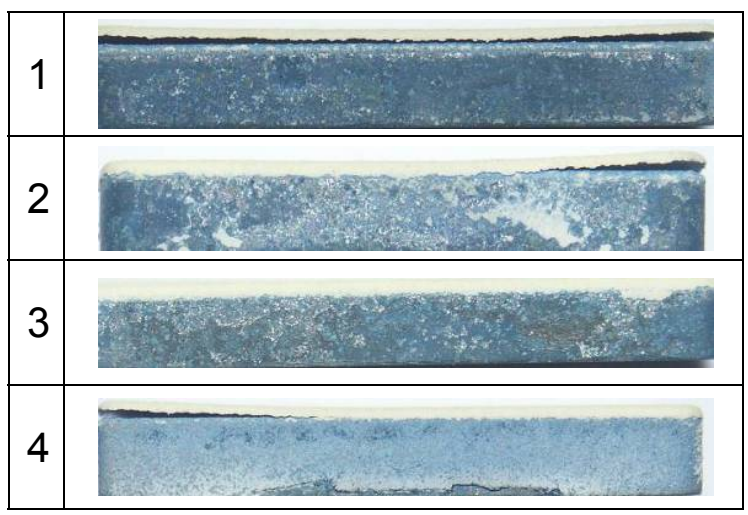

(b)

Low Stiffness

High Stiffness

Figure 45: APS/MCrAIY/RenéN5 coupon A, a) surface stiffness response contour map after 450 hours of isothermal exposure at $1100^{\circ} \mathrm{C}$ and b) edge spallation following 500 hours of isothermal exposure.

Although coupon A had failed following a cumulative total of 500 isothermal heat treatment hours, the coupon was once again placed in the furnace for a further 50 hour heat treatment where after which the YSZ completely separated from its substrate. Following large scale spallation failure coupon A was then stored for additional microstructural analysis of the fracture surface. Coupon $B$, unlike that of coupon $A$, displayed spallation failure along edge 1 upon its immediate removal from the furnace after 400 hours, Figure 46. 


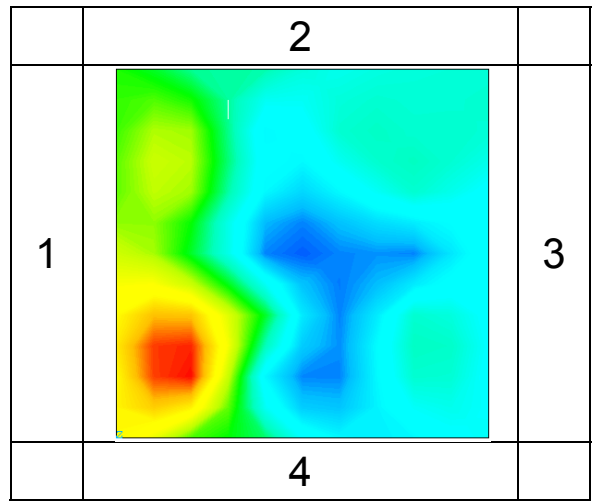

(a)

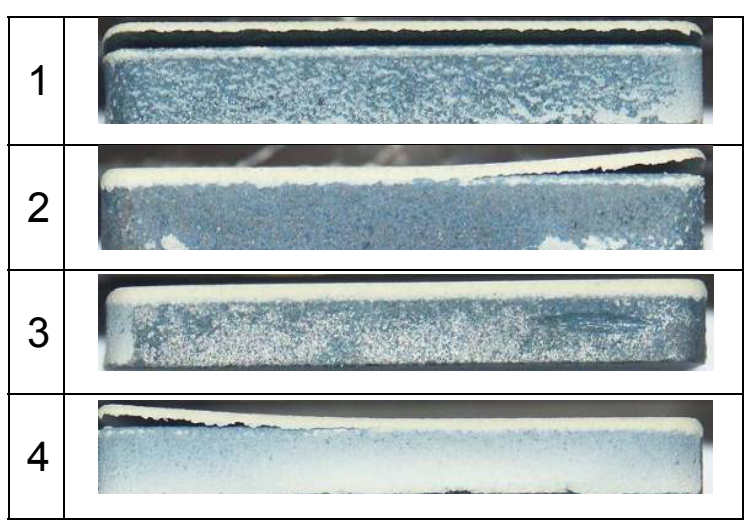

(b)

Low Stiffness

High Stiffness

Figure 46: APS/MCrAIY/RenéN5 coupon B, a) surface stiffness response contour map after 360 hours of isothermal exposure at $1100^{\circ} \mathrm{C}$ and b) edge spallation following 400 hours of isothermal exposure.

Following routine imaging and weighing, due to this failure, coupon B could no longer be evaluated using a micro-indentation technique and was thus chosen to be placed in storage for later microstructural and failure analysis. Coupons $\mathrm{C}$ and $D$, both intact post 400 hour thermal exposure underwent routine degradation evaluation using the multiple partial unloading procedure, thereafter both coupons were placed in sealed plastic bags awaiting further thermal exposures. Approximately one and a half weeks later, both coupons $C$ and $D$ were removed from storage where they were found to have spalled along edge 3 , coupon $C$ (Figure 47) and along edge 1, coupon D (Figure 48). 


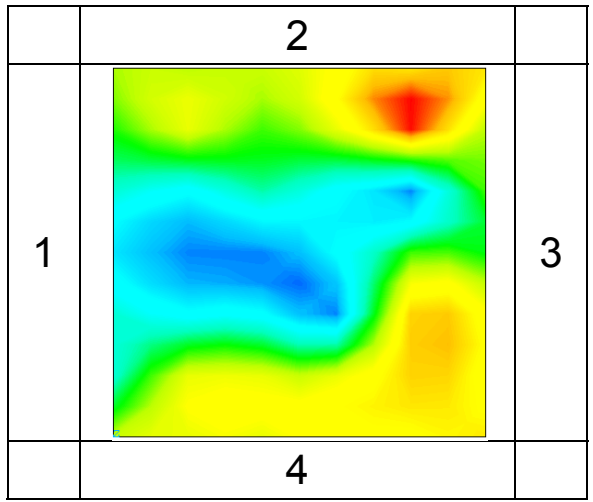

(a)

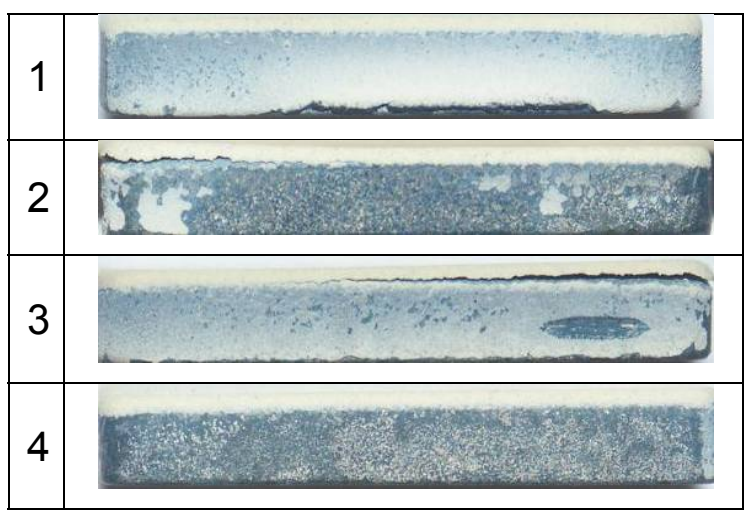

(b)

Low Stiffness

High Stiffness

Figure 47: APS/MCrAIY/RenéN5 coupon C, a) surface stiffness response contour map after 400 hours of isothermal exposure at $1100^{\circ} \mathrm{C}$ and b) desktop edge spallation 1.5 weeks after test completion.

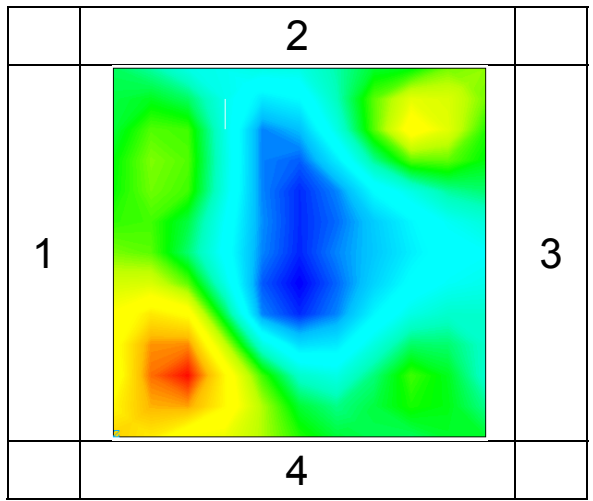

(a)

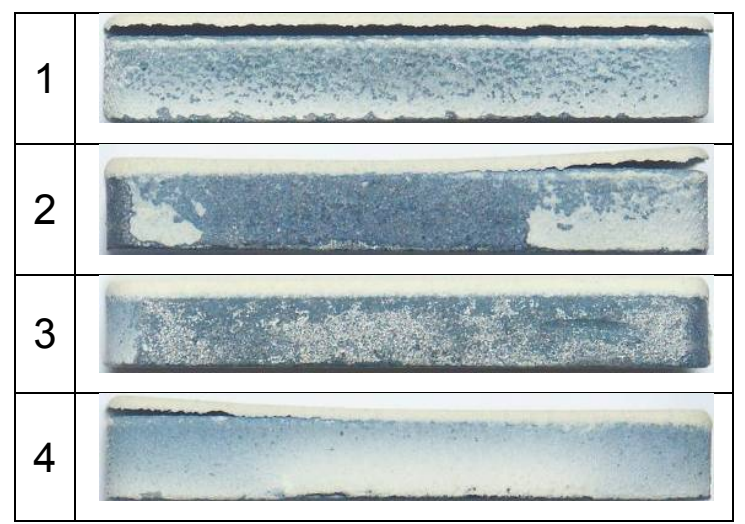

(b)

Low Stiffness

High Stiffness

Figure 48: APS/MCrAIY/RenéN5 coupon $D$, a) surface stiffness response contour map after 360 hours of isothermal exposure at $1100^{\circ} \mathrm{C}$ and b) edge spallation following 400 hours of isothermal exposure.

The contour color maps generated after 450 isothermal hours for coupon A, 360 isothermal hours for coupon B and 400 isothermal hours, for both coupon $\mathrm{C}$ and $\mathrm{D}$ displayed direct correlations to the locations of initial spallation failure. 
Denoted in red, regions having higher measured unloading slopes (shown above and further detailed in the Appendix) draw parallel to the edge spallation sites. The consistency of the contour color maps to the resulting regions of eventual spallation signifies a direct relationship between the areas having higher measured unloading surface stiffness response and eventual spallation failure location.

\subsubsection{Cyclic Exposure}

Coupons $E, F, G$ and $H$ were cyclically exposed to ambient air high temperature environments for a cumulative total of 400 and 440 hours at $1100^{\circ} \mathrm{C}$. The accumulated cycles at which all four cyclically exposed coupons failed varied from 400 (coupons $E$ and $H$ ) to 440 (coupons $F$ and E) total cycles. Spalling first, coupon $\mathrm{E}$ was exposed to a total of 400 cycles. Failure of this coupon did not occur immediately upon cooling to room temperature but rather two days later, following routine sample imaging and weighing along edge 1, Figure 49.

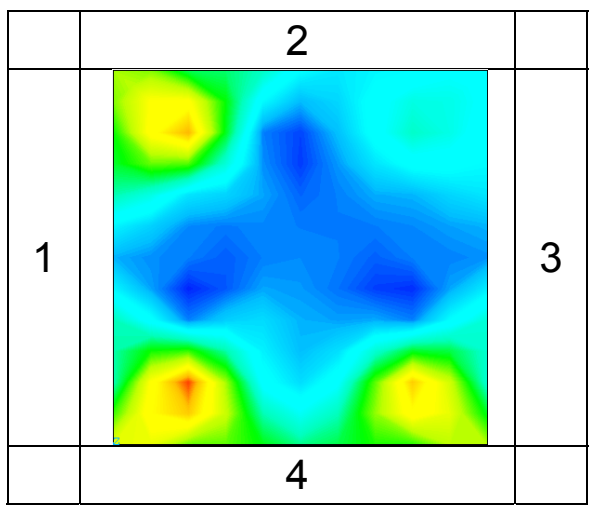

(a)

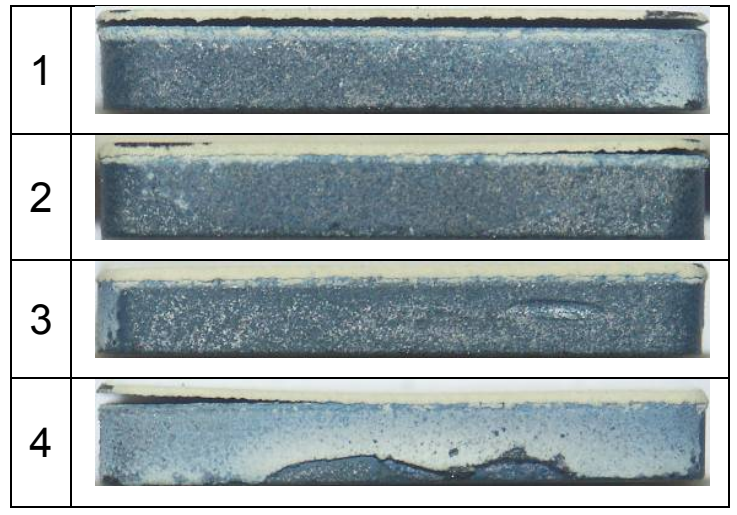

(b)

Low Stiffness

High Stiffness

Figure 49: APS/MCrAIY/RenéN5 Coupon E, a) surface stiffness response contour map after $\mathbf{3 6 0}$ thermal cycles and b) edge spallation following $\mathbf{4 0 0}$ thermal cycles.

Failing after exposure to 440 cycles, coupon F's YSZ completely separated from its super alloy substrate, Figure 50. Due to this, no direct correlation between indentation results and failure pattern could be made, yet some correlation 
between the contour color map and TBC spallation failure pattern can be seen at the corner of edges 1 and 4, Figure 50 (b).

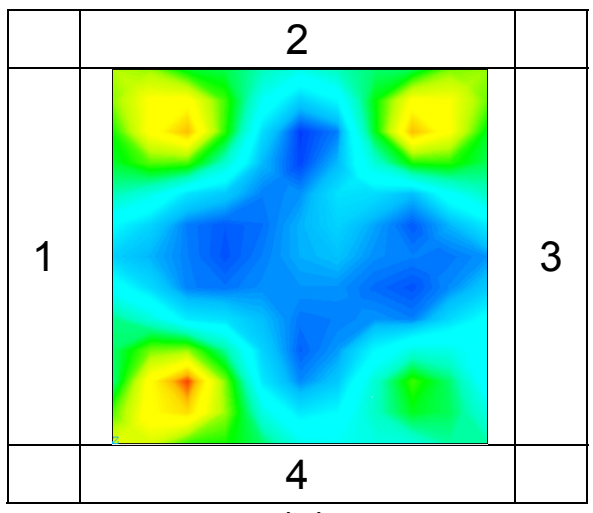

(a)

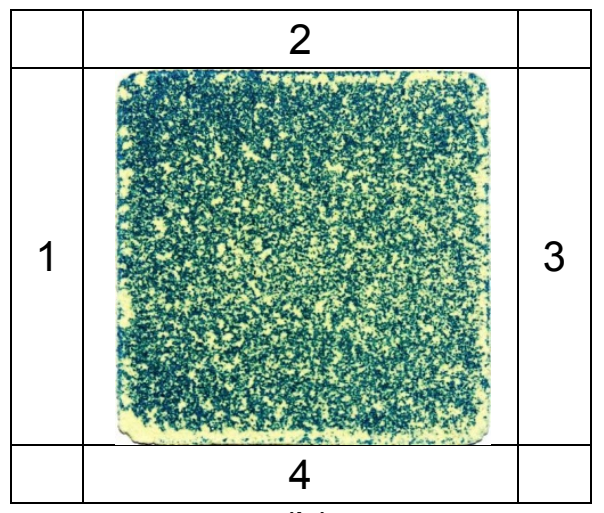

(b)

Low Stiffness

High Stiffness

Figure 50: APS /MCrAIY/RenéN5 Coupon F, a) surface stiffness response contour map after 400 thermal cycles and b) complete debonding of the APS YSZ along the TGO/metal interface after $\mathbf{4 4 0}$ thermal cycles.

Also spalling after 440 total cycles, Coupon $\mathrm{E}$ experienced a fracturing failure between the corners of edges 1 and 4, Figure 51 . This failure pattern was unusual for the APS/MCrAIY/RenéN5 TBCs and only occurred on this coupon.

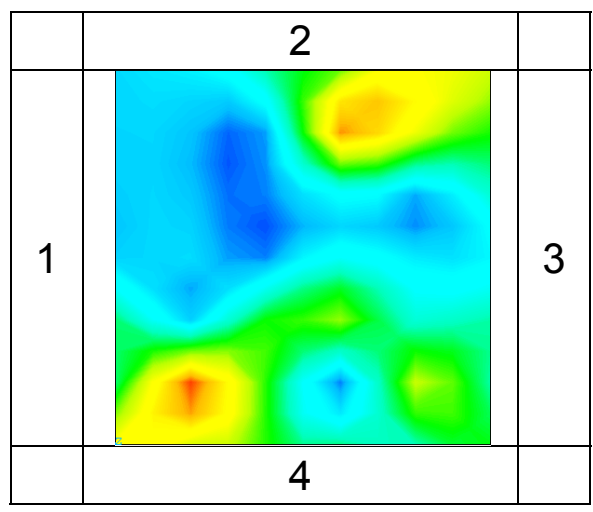

(a)

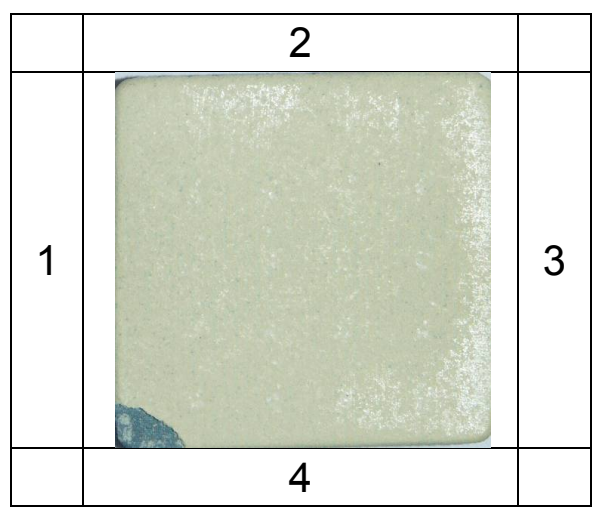

(b)

Low Stiffness

High Stiffness

Figure 51: APS /MCrAIY/RenéN5 Coupon G, a) surface stiffness response contour map after $\mathbf{4 0 0}$ thermal cycles and b) edge spallation following $\mathbf{4 4 0}$ thermal cycles. 
Coupon $\mathrm{H}$, the only EB-PVD/MDL/RenéN5 TBC in this study, was found to experience a similar failure pattern to coupon $G$ and fractured at the corner of edges 1 and 4 as well, Figure 52. Following a similar procedure to that of the isothermally exposed coupons, no samples were exposed to any additional thermal loading post spallation failure.

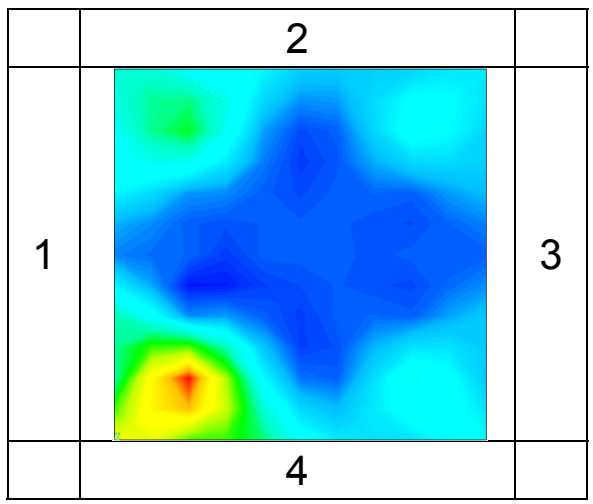

(a)

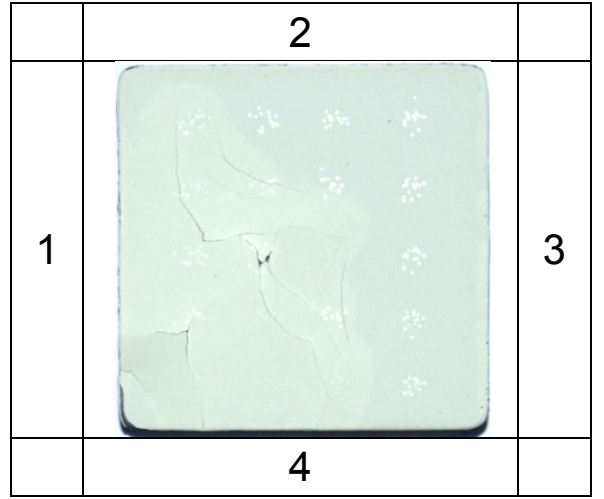

(b)

Low Stiffness

High Stiffness

Figure 52: EB-PVD/MDL/RenéN5 Coupon $\mathrm{H}$, a) surface stiffness response contour map after 600 thermal cycles and b) extensive spallation after 640 thermal cycles.

Demonstrating, as did the isothermally exposed coupons, contour color maps generated after 360 cycles for coupon E, 400 cycles for both coupon $\mathrm{G}$ and 640 cycles for coupon $\mathrm{H}$ display direct correlations with the locations of initial spallation failure. Excluding coupon $F$, for which no definite failure location has currently been identified, regions having higher measured unloading slopes (shown above and further detailed in the Appendix) coincide with observed edge spallation failure sites.

\subsubsection{Discussion}

The unloading slope averages obtained following each heat treatment did not continuously increase along cumulative thermal exposure, but rather steadily decrease, Figure 53. 


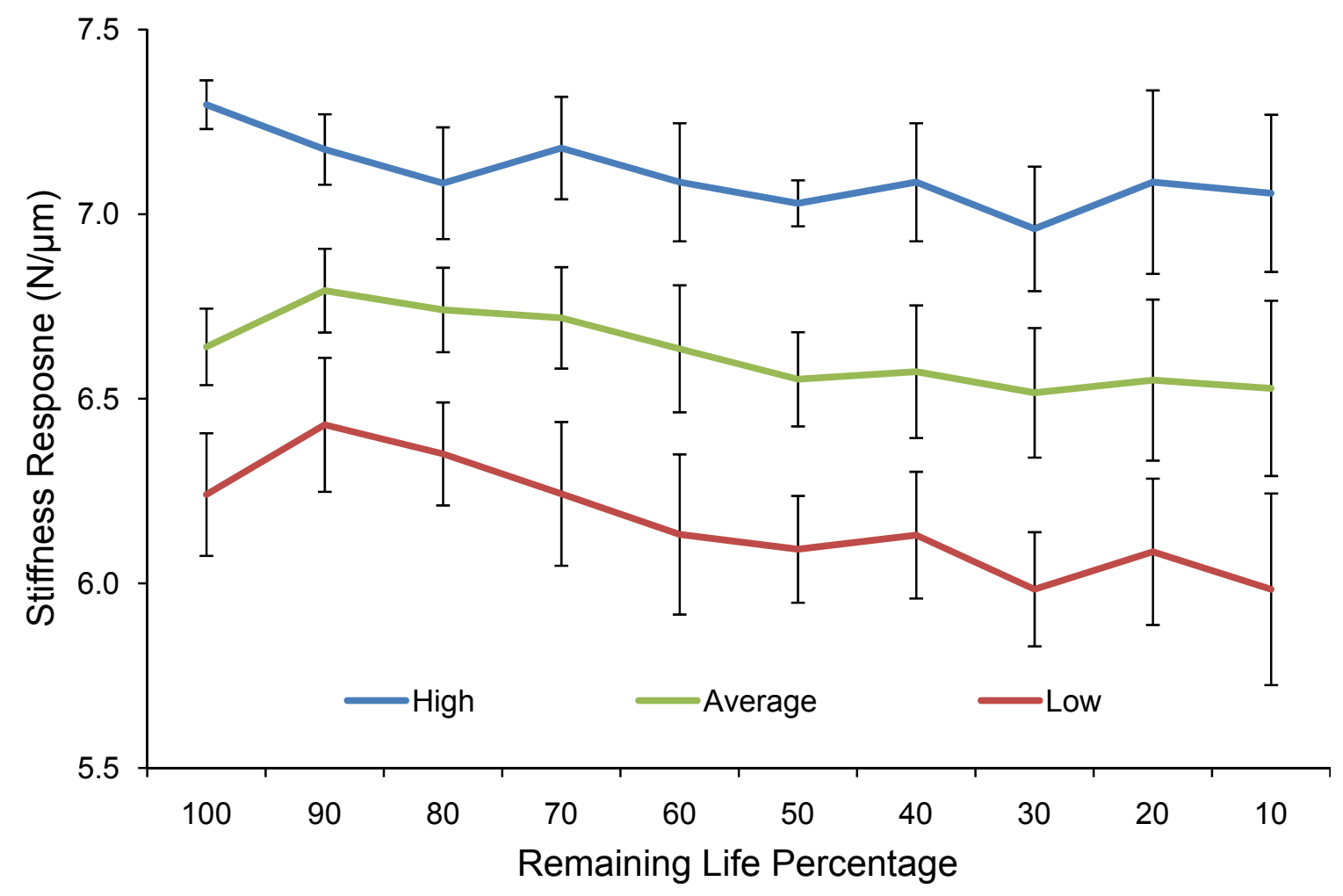

Figure 53: Average APS TBC high, low and average surface stiffness responses along TBC life percentage with $1 \sigma$ (standard deviation) shown.

This overall reduction in unloading stiffness is the result of several coating degradation mechanisms most notably cracking. However, displaying a trend opposite to that of the average unloading stiffness response, standard deviation within these data sets was found to increase with cumulative high temperature exposure, Figure 54. 


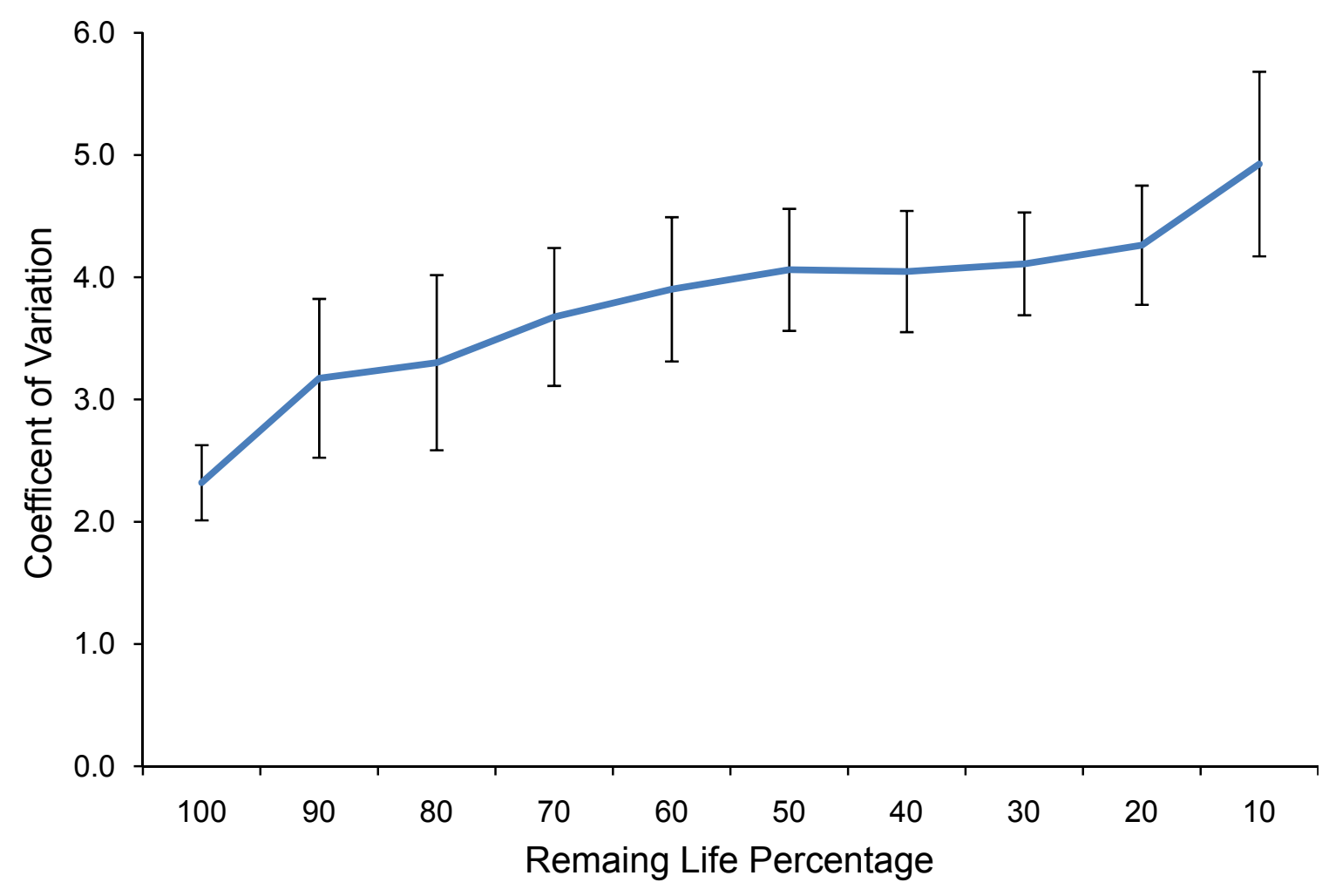

Figure 54: Average APS TBC coefficient of variation in surface stiffness response measurement with $1 \sigma$ (standard deviation) shown.

Described as the ratio between standard deviation and mean, Equation (15), coefficient of variation along thermal cycles is shown to increase indicating the existence of a greater division between low and high residually stressed regions within the TBC system [117]. Furthermore, all locations within a single TBC system have been subjected to the same thermal environments and thus only the variations in residual stress across the coupon are capable of producing this effect.

$$
C_{v}=\frac{\sigma_{k}}{\bar{y}_{k}} * 100
$$

Similar trends have also been observed by various non-destructive evaluation techniques as well, where TGO residual stress measurements acquired through photo-stimulated luminescence piezospectroscopic (PLPS) techniques show a clear two phase trends where an initial increase in residual compressive stress within the TGO is followed by a sharp decrease with increasing standard deviation in data sets, Figure 55 [139]. 


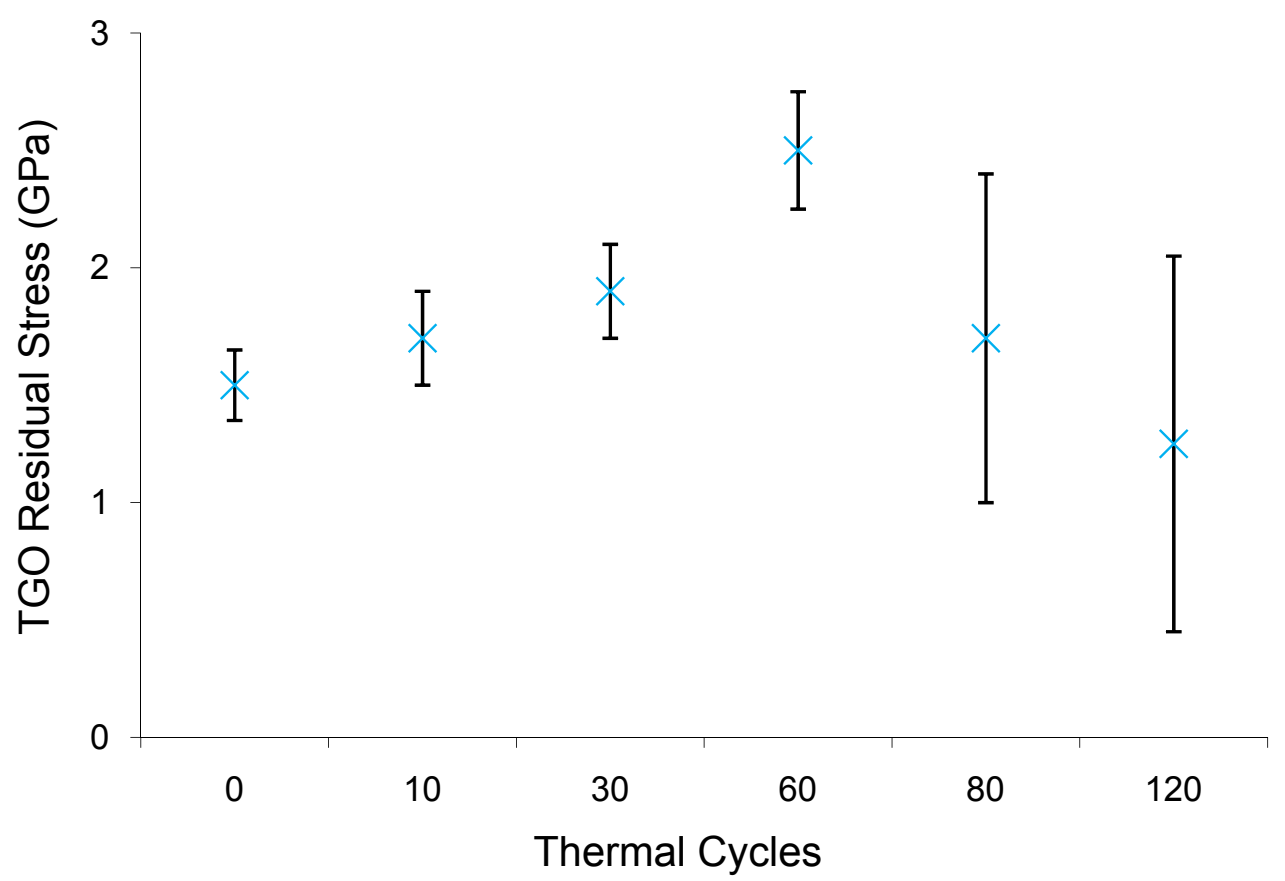

Figure 55: Average TGO residual stress along thermal exposure acquired by PLPS methods [139].

Furthermore, cross-sectional scanning electron microscope (SEM) imaging of a failed EB-PVD/MDL/RenéN5 coupon displayed those regions having a higher measured surface stiffness response prior to failure experience additional rumbling/roughening at the YSZ/TGO/BC interface, Figure 56.

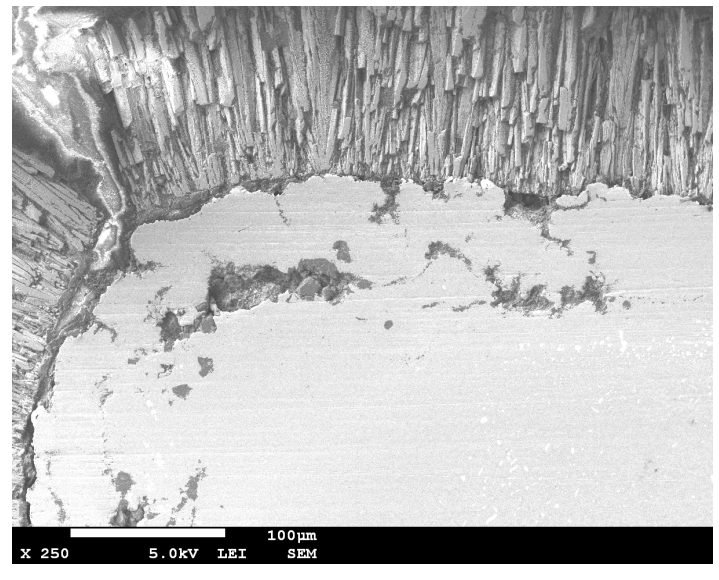

(a)

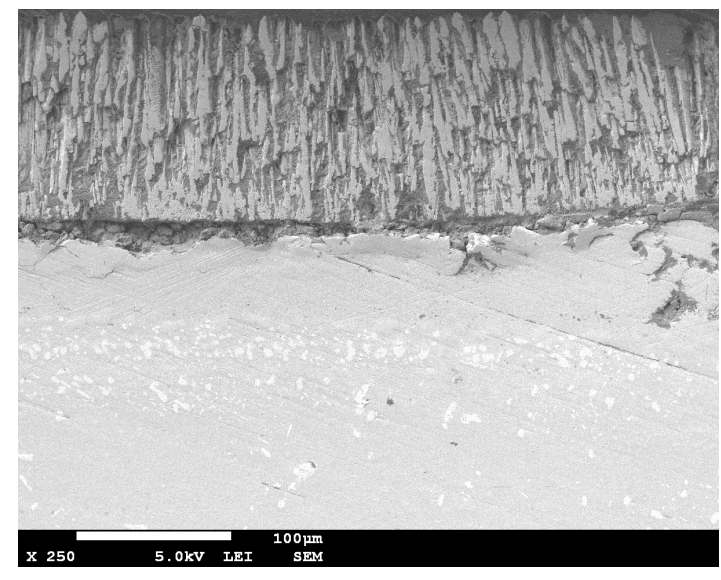

(b)

Figure 56: EB-PVD/MDL/RenéN5 coupon edge cross sectional scanning electron micrographs after 640 isothermal cycles, a) high and b) low residually stressed region. 
A direct comparison between these two images reveals a substantially greater amount of oxidation in Figure 56 (a) than that of image Figure 56 (b). Moreover, Figure 56 (b)'s YSZ/TGO interface appears to be more uniform than that of the nonlinear geometry found in Figure 56 (a). This less uniform interface geometry found in Figure 56 (a) is believed to be the source of high surface stiffness responses obtained in this region and ultimately led to the prediction of this location as that where failure would initiate. This confirms the notion that throughout Phase I the average residual stress within the TGO increases and is relatively uniform where in a small standard deviation between measurements is observed. Yet as the TBC system enters phase two and expedited cracking begins, average TGO residual stress tends to decrease yet variation within measurements is found to act opposite of that and increase [139].

The consistency of results previously presented demonstrates a direct correlation between failure location and regions having higher measured unloading surface stiffness response. The large residual stresses that develop within the TGO, YSZ and subsequent interfaces produce slight increases in unlading stiffness data obtained by the instrumented indentation system. Although throughout this study coating degradation was evaluated following each additional thermal exposure, this amount of testing may not have been necessary. Unloading stiffness values obtained at the later stages of TBC life demonstrated similar trends to those necessary for spallation failure prediction. Additionally, data is never compared to values obtained after the completion of a previous thermal exposure, eliminating the need for early cycle evaluation. Finally, some coupons used in this study did not experience immediate failure upon cooling to room temperature but rather days later. Isothermally exposed coupons $A, C$ and $D$ as well as cyclically exposed coupon $E$ did not experience failure for some time after thermal exposure. This desktop spallation failure phenomenon is credited to environmental moisture content and is difficult to control [141, 142]. Furthermore, spallation failure of all coupons thermally cycled in this study initiated from their free edges. This failure pattern is the result of shear stress concentrations occurring at the YSZ/TGO interface close to the free edge and has been shown 
to lead to interfacial cracking and delaminations in TBC systems $[143,144,145,146]$. Yet regardless the cause, the non-destructive load based indentation technique is capable of detecting those regions where failure will initiate.

\subsection{Microstructural Analysis}

The microstructural evolution within the YSZ/TGO/BC interface along ever increasing thermal loads has been documented by many yet the frequency at which coupons were sectioned has varied. In this research cross-sectional micrographs will be examined using current SEM techniques for oxide growth and interface rumbling at 40 isothermal and cyclic increments. Additionally, interface geometry across the coupon will be correlated to locations of measured increases in surface stiffness response. Through this, the cause for such increases is to be explained. However, due to the number of TBC coupons available a sample section technique was developed allowing sectioned samples to be thermally treated, sectioned and then exposed once again. The details of which are as follows: a) Prior to sectioning, all coupons are coated with an easily removed and flexible protective layer, purchased from Struers, this layer not only provides structural support but also prevents debris from imbedding within the coatings multiple layers throughout sectioning. b) Coupons are set in cycloalaphatic resin system and allowed to cure; this further enhances their structural support throughout the sectioning process. c) All coupons are sectioned using a low speed rotary saw equipped with diamond tipped wafering blades and may take up to 24 hours for coupons of this magnitude. d) Following sectioning, the coupon will be polished sequentially using $9,3,1,0.5$, and $0.1 \mu \mathrm{m}$ lapping films until a desired level of polish if reached. Furthermore, $0.02 \mu \mathrm{m}$ suspension fluid produces the surface finish required for SEM viewing. Throughout this process the coupon is periodically viewed under an optical microscope ensuring proper surface conditioning. However, although all precautions are taken to ensure no additional damage is induced throughout the sectioning process, failure is inevitable. Yet the level of induced damage is 
assumed to be constant across all samples and can be ignored. Ultimately, a library of TBC cross-sections at 40 cycle and isothermal increments is created. These sectioned coupons are then observed using a JEOL 7600F SEM with Oxford Inca EDS for microstructural evaluation.

Although experimental stress analysis techniques may be applied to TBC systems, these methods are costly and relatively undefined, thus parametric finite element modeling techniques have been applied to further describe stress relaxation behavior with various material compositions [147,148]. Additionally, analysis of the compressive residual stresses within TBC systems has lead to the development of a geometry dependent crack prediction model [149]. Following this, analytical models examining residual stresses were developed as a function of TGO thickness, thermal loading rates and thermal expansion coefficients under simulated cyclic thermal histories [150]. However, it is the undulating YSZ/TGO/BC interface, which produces the out-of-plane residual stresses responsible for in-service failure of TBC systems [93]. In this research several finite element models have been developed via experimentally obtained crosssectional scanning electron micrographs. Through a detailed analysis of the outof-plane tensile stresses produced upon cooling, a complete spallation failure prediction path has been developed in addition to causes for such delaminations occurring prior to a sufficiently thick oxide scale developing.

\subsubsection{Object Oriented Finite Element Method}

Research efforts in object oriented finite element modeling (OOFEM) techniques have allowed residual stresses within thermally cycled TBC systems to be estimated using true interface geometries [151]. Through this procedure experimentally obtained cross-sectional TBC micrographs may be transformed to finite element models along thermal exposure hours/cycles. Ultimately, a numerical simulation is employed to determine residual stress levels within and around the TBC's YSZ/TGO/BC interfaces as thermal loads are applied. Additionally, utilizing a "Photomerge" option in Adobe Photoshop CS ${ }^{\mathrm{TM}}$, multiple SEM micrographs spanning the length of a TBC cross section may be assembled 
forming a large, but highly detailed image. A partial/incomplete example of this can be found in Figure 57 where only 6 images are combined.

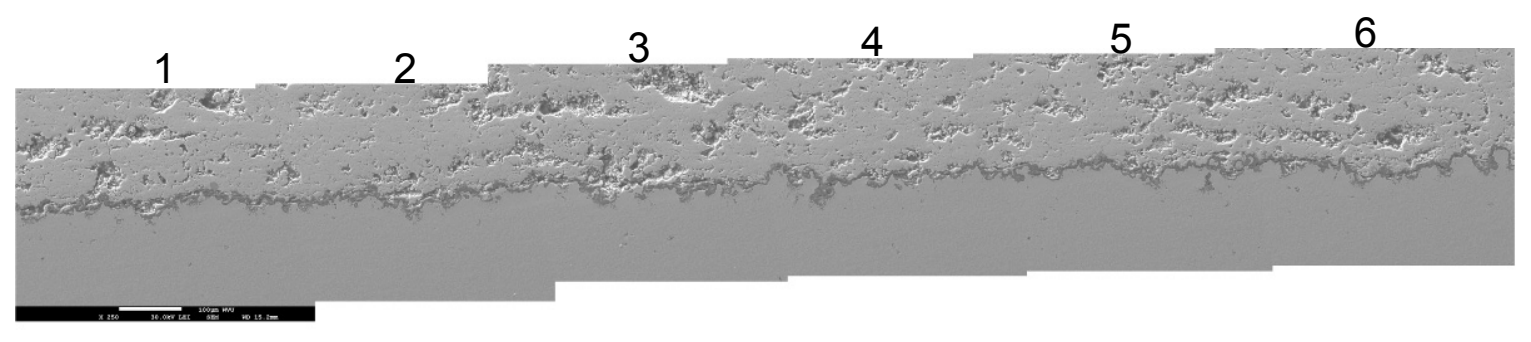

Figure 57: YSZ/TGO/BC microstructure geometry interface.

Through this process the full width of TBC cross-section may be evaluated numerically via finite element software. Following extraction of the TGO geometry via in house developed software, the remaining TBC system materials are added (TBC: $353 \mu \mathrm{m}, \mathrm{BC}: 209 \mu \mathrm{m}$ and Substrate: $3000 \mu \mathrm{m}$ ), Figure 58.

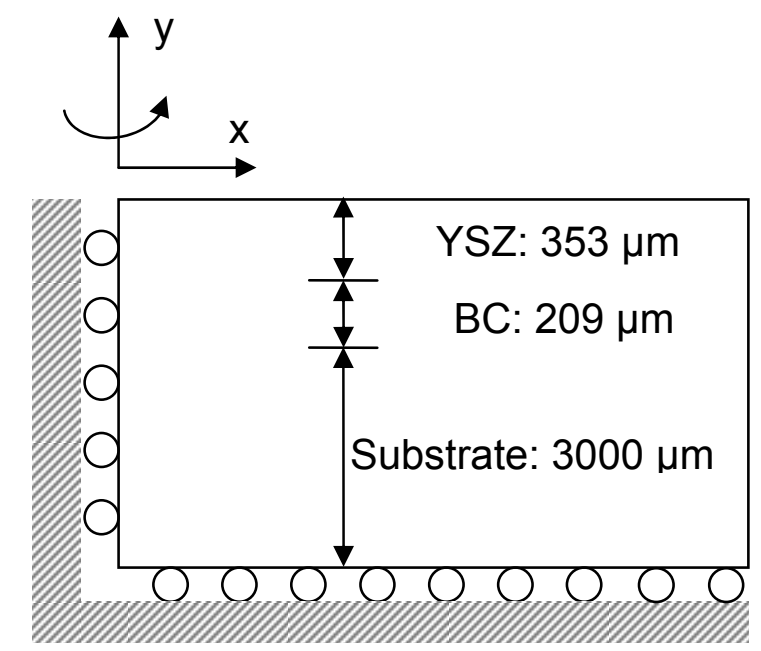

Figure 58: Object oriented finite element analysis model having a thickness of 12700 microns.

Material properties imposed on this model include the elastic modulus, Poisson's ratio, yield stress, tangent modulus and coefficient of thermal expansion for all layers, Table 3 [104]. 
Table 3: TBC system material properties [104].

\begin{tabular}{|c|c|c|c|c|}
\hline Material Property & YSZ & TGO & BC & Substrate \\
\hline Elastic Modulus (GPa) & 50 & 375 & 211 & 150 \\
\hline Poisson's Ratio & 0.1 & 0.25 & 0.3 & 0.25 \\
\hline Yield Stress (MPa) & 250 & 1000 & 422 & 841 \\
\hline Tangent Modulus (GPa) & 1 & 3.75 & 0.889 & 1.05 \\
\hline CTE $\left(10^{-6} /{ }^{\circ} \mathrm{C}\right)$ & 11 & 8 & 14 & 14.5 \\
\hline
\end{tabular}

Also, a Plane182 element is employed with axis symmetry and isotropic bilinear elastic-plastic material behavior. Finally, upon application of a temperature difference of $\Delta-1077^{\circ} \mathrm{C}$, the now complete TBC system reveals a stress contour plot in response to experimentally obtained YSZ/TGO/BC interface geometries, Figure 59.

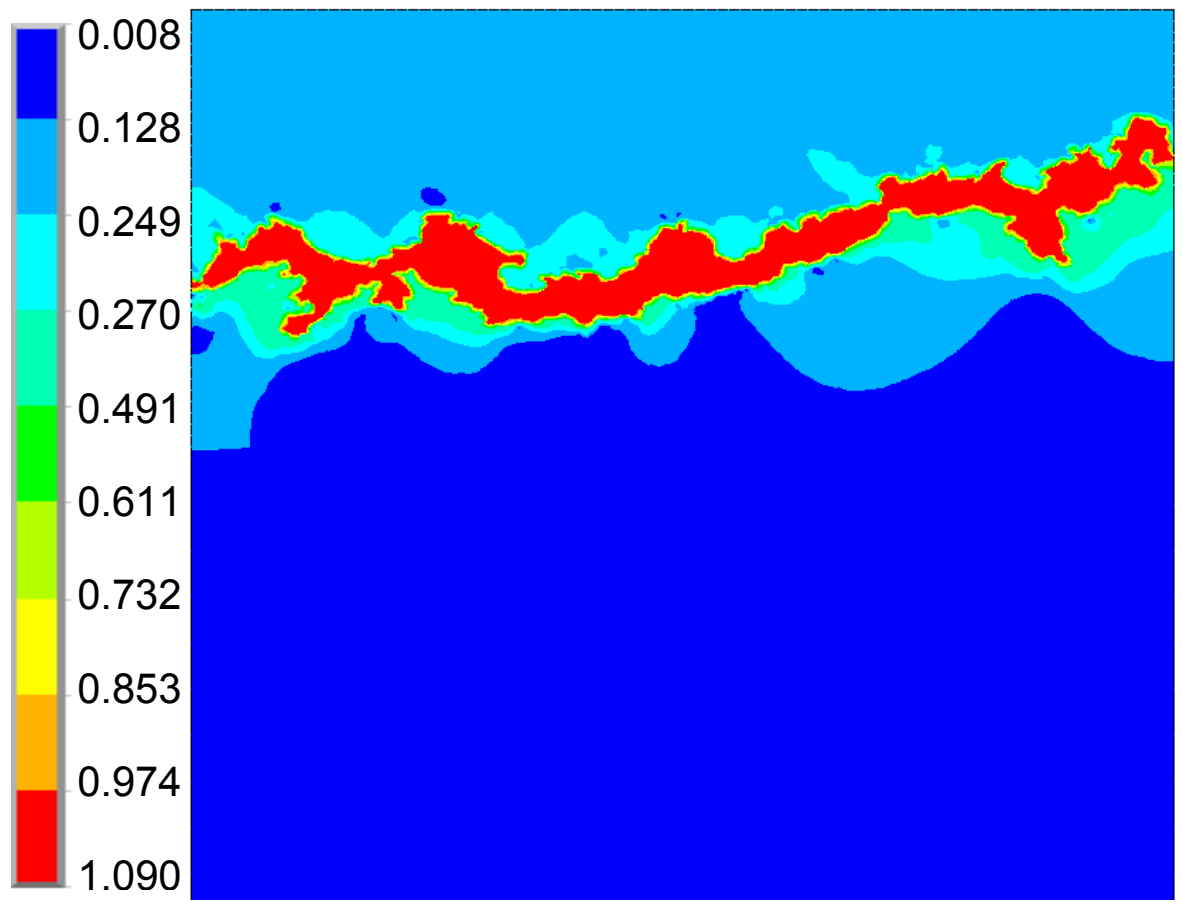

Figure 59: Object oriented finite element analysis Von Mises residual stress in GPa.

This preliminary result is shown to yield a maximum compressive residual stress within the TGO of $1 \mathrm{GPa}$. As this oxide layer continues to grow, the stresses produced in this region are expected to increase. Although this in-plane compressive residual stress is not the direct cause of spallation failure, it leads to out-of-plane tensile residuals stresses that do. The residual stresses extracted 
from this model will not only provide a comparison across thermal cycles but along the coupon's width as well. By this method it may be determined if boundary conditions along the coupon's edges are responsible for premature spallation failure.

\subsubsection{Experimental Failure Observations}

The investigated thermal barrier coating systems consisted of the single crystal Ni-base superalloy RenéN5 substrate, NiCoCrAlY bond coat and a $7 \mathrm{wt} \%$ yttria partially stabilized zirconia top coat applied by an air plasma-spray process. Atop the $1 / 8$ " thick by 1 " square substrate, bond coats and top coats were applied with thicknesses of 300 and $350 \mu \mathrm{m}$, respectively. Coupons were thermally cycled at temperatures of $1100^{\circ} \mathrm{C}$ in ambient air environments. Each thermal cycle consisted of heating with a rate of $1.2^{\circ} \mathrm{C} / \mathrm{s}$ followed by a dwell time at a maximum temperature of $1100^{\circ} \mathrm{C}$ for 40 hours, followed by a subsequent $1.2^{\circ} \mathrm{C} / \mathrm{s}$ cooling to room temperature. Thermally exposures were conducted in a horizontal, resistance coil tube furnace able to maintain a set temperature of $\pm 3^{\circ} \mathrm{C}$. The coupons were metallographically prepared by cold mounting the samples in epoxy resin to avoid additional damage that may occur during this process. Coupon section was performed with precision diamond wafer blades where after initial grinding with SiC lapping film was followed by final polishing being performed with colloidal silica suspension liquid down to $1 \mu \mathrm{m}$ grain size. The colloidal silica provided both a chemomechanical polishing (CMP) action and reduced subsurface damage. Microstructural investigations were carried out with a JEOL 7600F scanning electron microscope. All coupon cross-sections were sputter coated (AuPd) prior to viewing, this procedure was required to reduce the amount sample charging throughout imaging. All TBC coupons were supplied by the National Energy Technology Laboratory (NETL) a subsidiary of the United States Department of Energy (DOE).

Large scale spallation failure of all thermally cycled TBC systems initiated from the coupons edges, however prior to this event several microstructural oxide interface observations were made and are as follows: 
a. Although a majority of the numerical models developed to evaluate residual stresses and failure mechanisms of APS TBC systems enlist uniform TGO thickness, these geometrical assumptions are rarely experimentally observed, Figure 60.

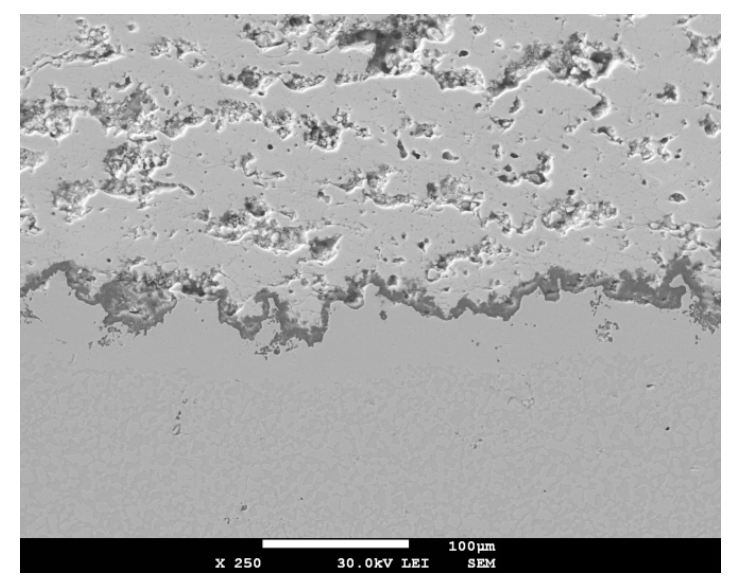

Figure 60: Non-uniform TGO thickness.

b. A result of bond coat roughness, YSZ/TGO/BC interface geometry resembles that of a sinusoidal wave and would be applicable to model in this manor, Figure 61.

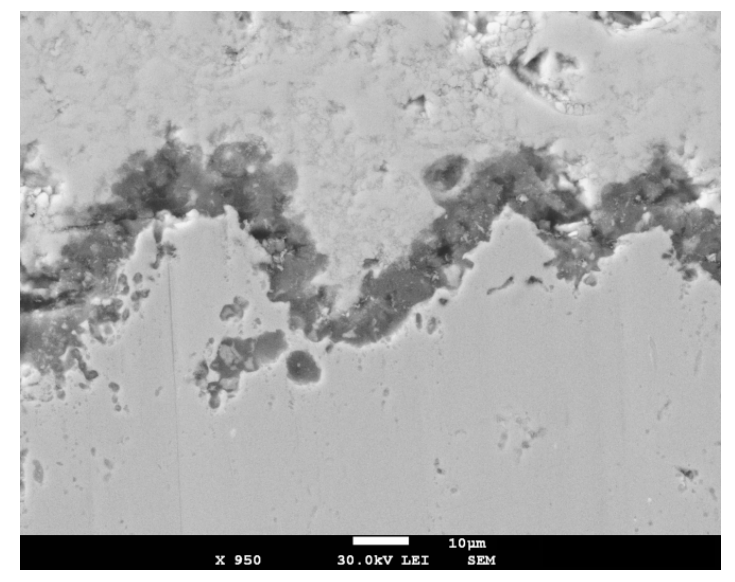

Figure 61: TGO sinusoidal wave pattern.

c. Within the TGO wave several thickness variation trends were observed, however may be separated into three categories: i) uniform thickness across the wave, ii) greater oxide thickness at the apex (Figure 62) and iii) greater oxide thickness at the base. 


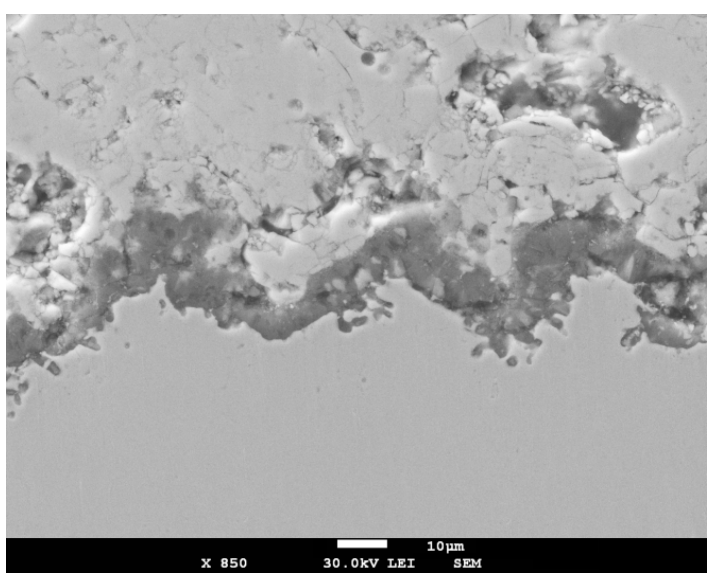

Figure 62: TGO wave thickness variability.

d. Although micro-cracks were observed in several locations, those taking place within the thermally grown oxide and not its interfaces occurred at the oxide wave's midline, Figure 63.

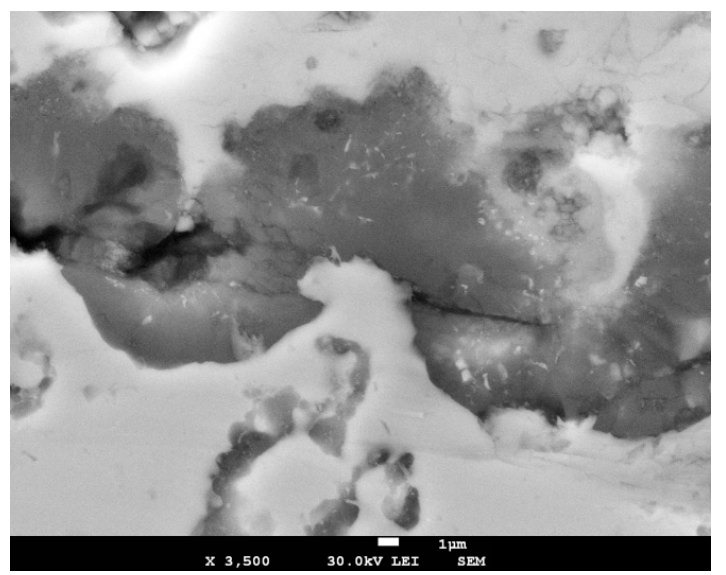

Figure 63: Observed TGO thickness variability.

e. Interface crack coalesce generally followed a similar failure progression to that previously established by various researches [86] where: i) micro cracks develop at the apex TGO/BC interface wave, ii) these cracks then propagate and extended through the TGO at the wave mid line and iii) emerging on the opposite side of the TGO, these micro-cracks then lengthen and extend to the YSZ just above the TGO wave's base, Figure 64. 


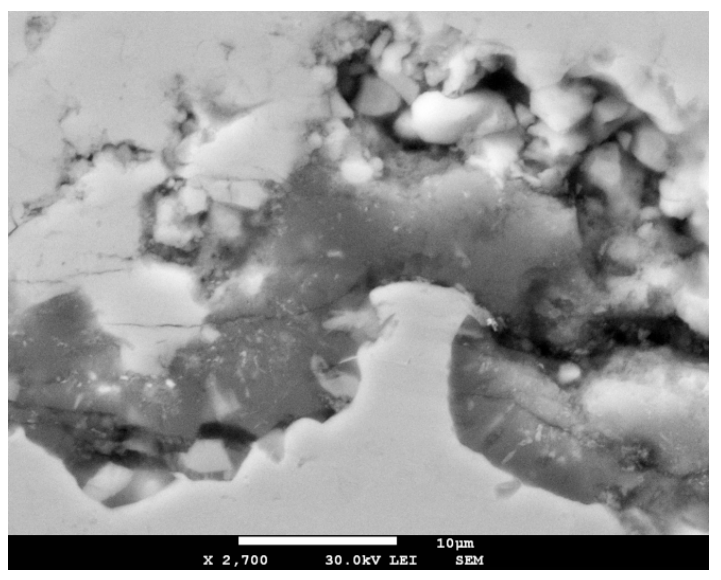

Figure 64: YSZ/TGO/BC interface failure pattern.

With these observations a parametric numerical model has been developed to explain the more commonly observed failure patterns. Specifically, detail the micro-cracking progression described in item (e) using a variable TGO thickness model. Also, establish a relative residual stress state at the YSZ/BC/TGO interface using the various non-uniform TGO thickness geometries presented in item (c). Moreover, as several uniform TGO thickening models have been developed researchers [101], a final objective of this research is the determine the relationship variable TGO geometries have on interface residuals stress states.

\subsubsection{Parametric Design}

In this parametric modeling design, a sinusoidal YSZ/TGO/BC interface was assumed. As previously stated, this geometry was implemented to more precisely simulate the experimentally observed YSZ/TGO/BC interfacial microstructure. To accomplish this, sine wave functions were implemented for both the TGO/BC as well as the YSZ/TGO interface, Figure 65. 


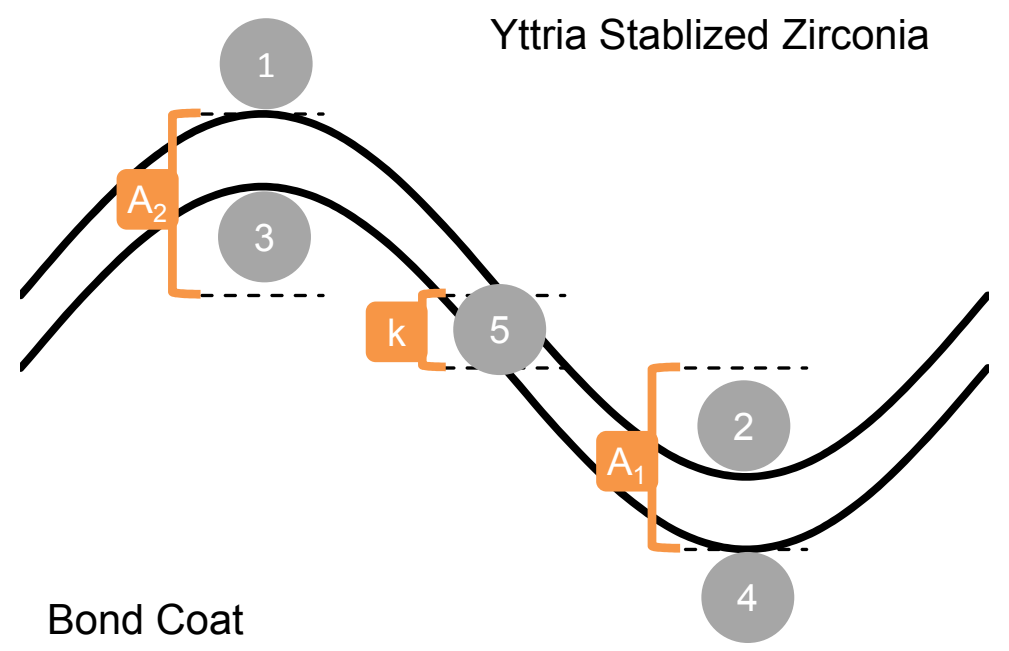

Figure 65: Thermally grown oxide modeling parameters.

Defined by amplitude $\left(A_{i}\right)$, wave length $(\lambda)$ and offset $(k)$, functions for both lines are developed using Equations (16) and (17). From these it can been seen that if both the TGO/BC interface amplitude $\left(A_{1}\right)$ and YSZ/TGO amplitude $\left(A_{2}\right)$ are equal, a uniform TGO model is generated with a thickness of offset $(k)$.

$$
\begin{gathered}
y_{T G O / B C}=A_{1} \sin \left(\frac{2 \pi}{\lambda} x\right) \\
y_{Y S Z / T G O}=A_{2} \sin \left(\frac{2 \pi}{\lambda} x\right)+k
\end{gathered}
$$

Although models having uniform TGO thickness have been found capable of reasonably predicting TBC spallation patterns [102] rarely are homogeneous TGO layers found in APS TBC systems. As previously presented, modeling this layer with a variable thickness is more practical and will ultimately lead to a more detailed failure analysis. To accomplish this, the amplitude of both interfaces, TGO/BC and YSZ/TGO, may not be equal. Furthermore, although it may be simple to vary interfacial geometry when modeling, the TGO/BC interface roughness is the result of several manufacturing parameters [152] and largely influenced by oxygen ingress, thermal cyclic temperature and surface morphology among others [68]. As a result, oxidation rates across even a single TBC coating may vary, leading to the development of non-uniform oxide scales. 
To simulate this effect a constant TGO/BC interface amplitude of $5 \mu \mathrm{m}$ was chosen, and only YSZ/TGO amplitude $\left(A_{2}\right)$ is varied. Additionally, although composed of several parameters $\left(A_{1}, A_{2}\right.$ and $\left.k\right)$, variations in TGO geometry may defined as the ratio between thicknesses at the apex and base of the sinusoidal wave, Equation (18), thus combining all geometric parameters into one quantifiable value.

$$
\beta=\frac{\left.t_{T G O}\right|_{\lambda / 4}}{\left.t_{T G O}\right|_{3 \lambda / 4}}=\frac{A_{2}-A_{1}+k}{A_{1}-A_{2}+k}
$$

Conducting a parametric numerical analysis along $\beta$ will establish any correlation that may exist between geometry and residual stress state at the YSZ/TGO/BC interface, Table 4.

Table 4: Numerical TGO model design parameters.

\begin{tabular}{|c|c|c|c|c|c|}
\hline Model & $\lambda$ & $A_{1}$ & $A_{2}$ & $k$ & $\beta$ \\
\hline Number & $(\mu \mathrm{m})$ & $(\mu \mathrm{m})$ & $(\mu \mathrm{m})$ & $(\mu \mathrm{m})$ & $(\mu \mathrm{m} / \mu \mathrm{m})$ \\
\hline 1 & 80 & 5 & 3.0 & 8.0 & $3 / 5$ \\
\hline 2 & 80 & 5 & 3.5 & 6.5 & $5 / 8$ \\
\hline 3 & 80 & 5 & 4.0 & 5.0 & $2 / 3$ \\
\hline 4 & 80 & 5 & 4.5 & 3.5 & $3 / 4$ \\
\hline 5 & 80 & 5 & 5.0 & 2.0 & $1 / 1$ \\
\hline 6 & 80 & 5 & 5.5 & 3.5 & $4 / 3$ \\
\hline 7 & 80 & 5 & 6.0 & 5.0 & $3 / 2$ \\
\hline 8 & 80 & 5 & 6.5 & 6.5 & $8 / 5$ \\
\hline 9 & 80 & 5 & 7.0 & 8.0 & $5 / 3$ \\
\hline
\end{tabular}

From this a more detailed TBC failure progression may be established and also provide explanation for experimentally observed spallation patterns detailed in the previous section. Finite element simulations were carried out using an axissymmetric TBC model in ANSYS ${ }^{\mathrm{TM}}$ V11.0 containing all layers with thicknesses of each being $353 \mu \mathrm{m}, 209 \mu \mathrm{m}$ and $2700 \mu \mathrm{m}$ for the YSZ, BC and Substrate, respectively $[147,148]$. To avoid singularity and obtain a semi-infinite domain a width of $500 \mu \mathrm{m}$ is used with nodes along the axis of symmetry and those along the base constrained to allow movement the length of these axes only, Figure 66. 


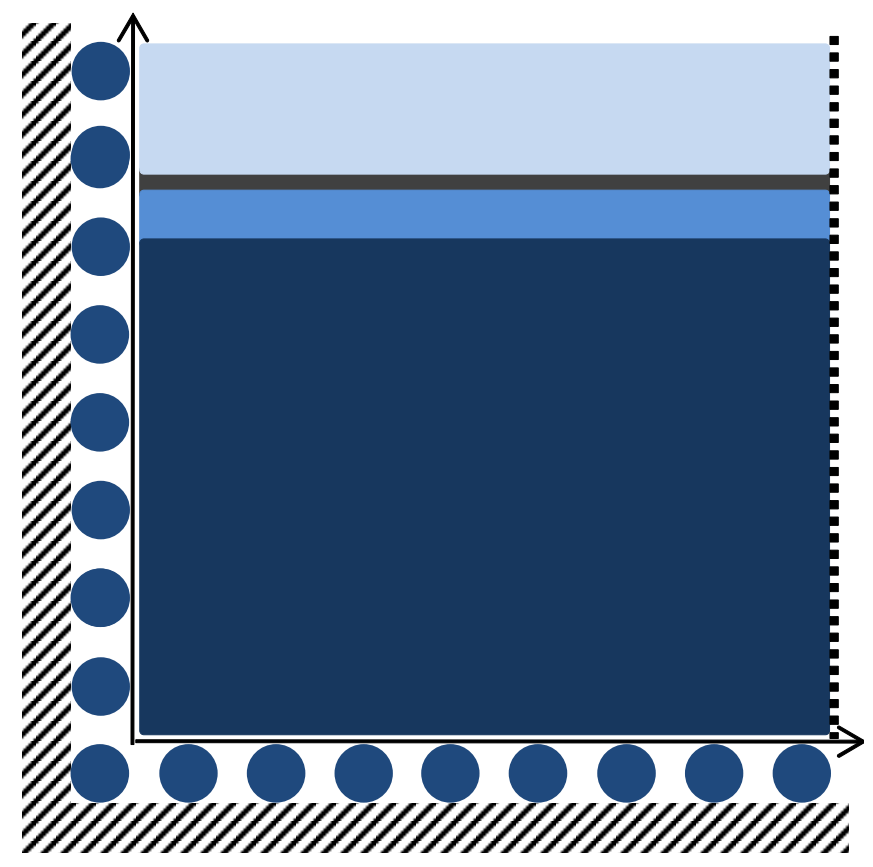

Figure 66: Boundary conditions applied to the numerical TBC model.

Enhancing resolution of the resultant stress distribution, minimum element sizes of $0.7 \mu \mathrm{m}$ were implemented within the TGO and increased to a value of 3 $\mu \mathrm{m}$ when entering the $\mathrm{YSZ}$ and $\mathrm{BC}$, Figure 67.

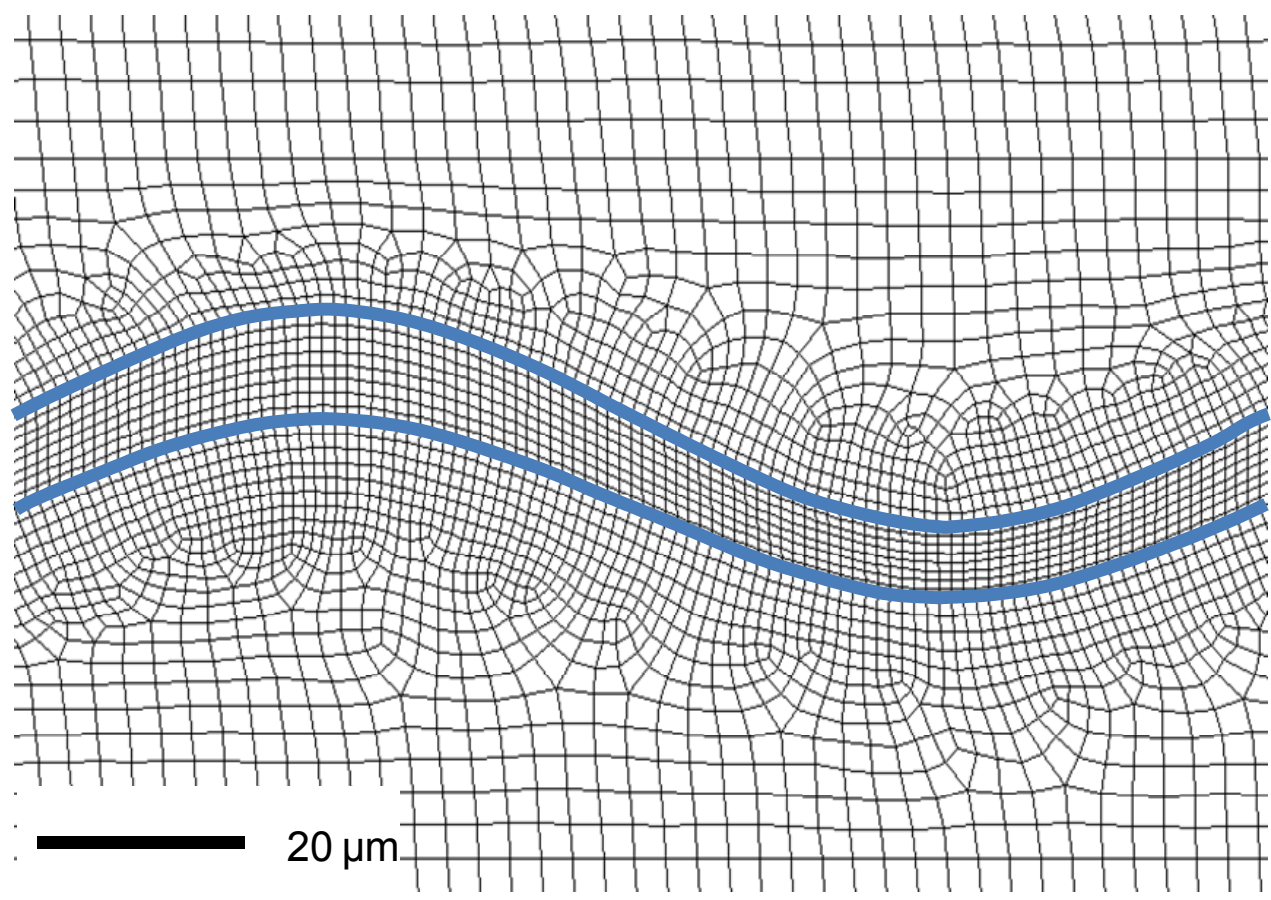

Figure 67: YSZ/YGO/BC interface mesh resolution. 
Required to induce residual stress, a reduction in thermal load from $1100^{\circ} \mathrm{C}$ to $23^{\circ} \mathrm{C}$ is applied to the TBC system with a $\Delta \mathrm{T}$ of $-1077^{\circ} \mathrm{C}$. These temperatures were chosen due to the condition of which the experimental coupons were thermally cycled. Finally, a bilinear isotropic hardening material model has been employed, as a result both elastic and elastic-plastic behavior material behavior is considered, Table 3 [104].

\subsubsection{Discussion}

Due to the complexity of TBC interface overall residual stress states along $\beta$, only the five locations where micro-cracking was consistently observed are presented, Figure 65. Of these five locations, four reside at the apex and base of the YSZ/TGO (nodes 1 and 2) and TGO/BC (nodes 3 and 4) interfaces, in addition to one at the TGO wave's midline (node 5). Although in-plane residual stress states have been reviewed, their overall magnitude was found to be well bellow that needed to cause compressive failure. Furthermore, recent studies indicate that it is not the in-plane but rather that out-of-plane tensile stresses that are directly responsible for micro-cracking and spallation failure patterns in TBC systems [86]. Due to this only the out-of-plane tensile stresses at interface nodes 1, 2, 3 and 4, as well as shear stress at node 5 are presented bellow.

The out-of-plane stresses developing at the YSZ/TGO interface's apex (node 1) are found to be tensile as $\beta$ approaches zero, however transitions to compression as it exceeds 1, Figure 68. Conversely, stresses developing at the interface's base (node 2) are inversely proportional to those at the apex, where tensile stresses at this location exist only as $\beta$ exceeds 1 , Figure 68. These contrasting states of stress at nodes 1 and 2 acts to prevent localized cracking that may develop at either from propagating beyond these points. As a result, it is not surprising that large scale spallation failure along this interface is rarely observed. Furthermore, this contrast in residual stress state exists between locations 3 and 4 as well, where the out-of-plane stresses at node 3 increase symmetrically about $\beta=1$, yet residual stress states at location 4 are found to be uniformly compressive along all $\beta$, Figure 68. These compressive stresses 
produced at node 4 are not conducive to micro-crack development and thus those developing at the location 3 typically do to propagate along the TGO/BC interface and result in large scale spallation failure either.

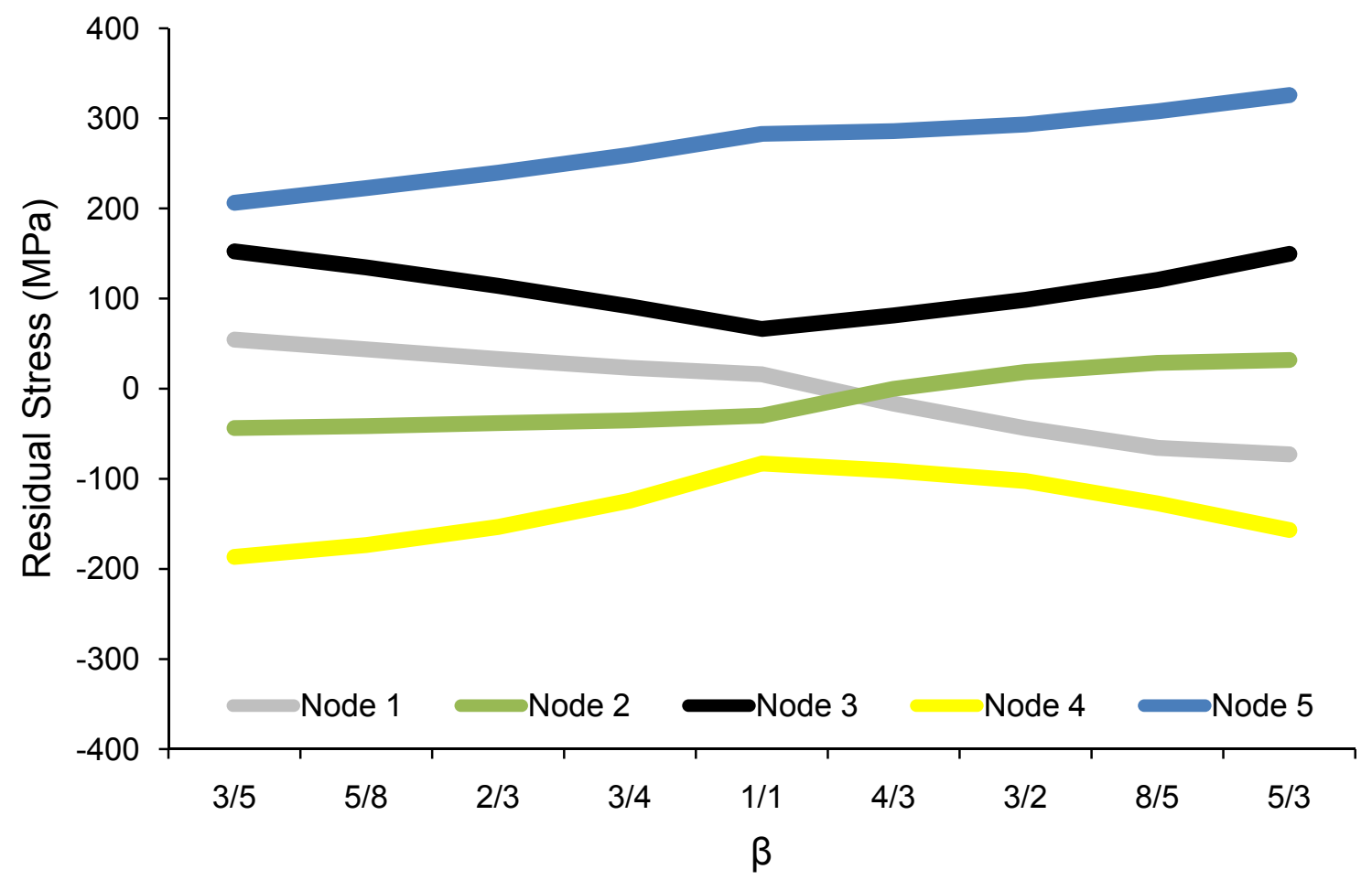

Figure 68: Finite elemental node residual stress state along $\beta$.

Unlike that of nodes 1, 2 and 3, cracks developing at location 5 are not easily explained through review of the out-of-plane residual stresses acting in this region as a compressive stress state exist along all $\beta$. However, the cause of localized cracking in this region becomes evident upon review of the systems maximum shear stress magnitude and location (node 5), Figure 68 and Figure 69.

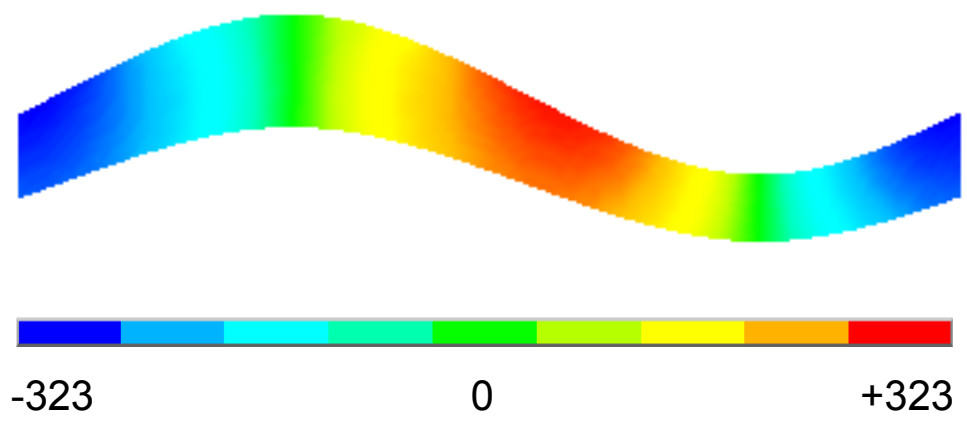

Figure 69: Oxide scale shear stress profile (MPa). 
Furthermore, according to the Rankine criterion for brittle materials, failure takes place when the maximum principal stress at a point inside the material reaches a value equal to the tensile strength $\left(\sigma_{y}\right)$ as found in a simple tension test [153]. Thus according to this criterion, the principle stresses acting node 5 (50.8 $\mathrm{MPa},-1.10 \mathrm{GPa}$ and $-1.13 \mathrm{GPa}$ ) as $\beta$ approaches a maximum exceed that of the TGO's yield stress (1 GPa) and initiate failure. From this, a now complete spallation failure pattern becomes evident as $\beta$ exceeds 1 . More specifically, as the out-of-plane tensile residual stress at node 2 and 3 increase simultaneously with shear and $\beta$, a crack path of least resistance is generated, Figure 70.

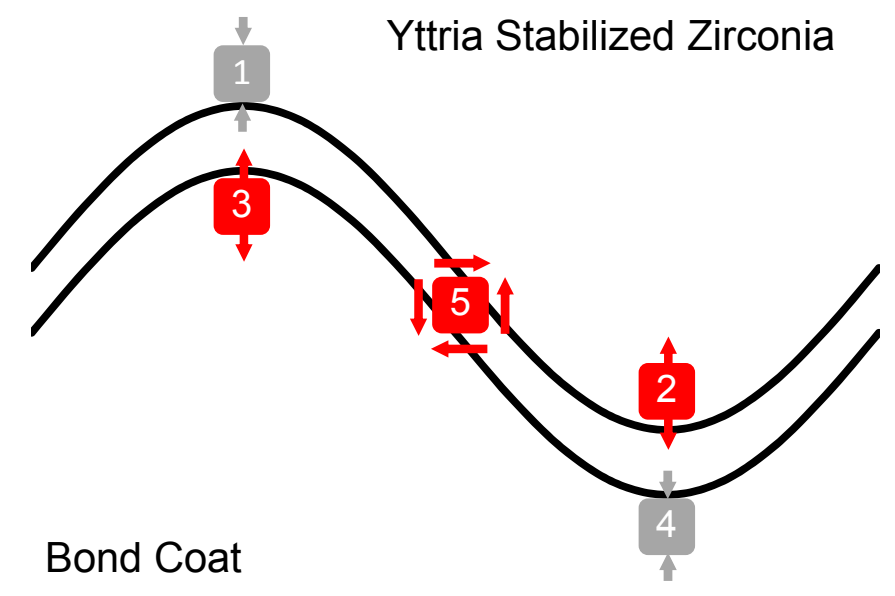

Figure 70: Oxide scale failure inducing stress pattern created as $\beta$ approaches a maximum.

Inversely, as $\beta$ approaches zero out-of-plane tensile stresses only occur at locations above and bellow the TGO wave apex (nodes 1 and 3 ), and thus are not advantageous for micro-crack propagation. Finally, although micro-cracks at nodes 1, 2 and 3 may exist due to tensile stresses developing in these regions at various $\beta$, it is not astonishing that large scale spallation failure patterns along either interface are seldom found. The contrasting out-of-plane residual stresses' acting at these locations prevents the propagation and coalescence of microcracks. As a result, large scale micro-spallation is more likely to follow a failure pattern in which cracks developing at the peak of the TGO/BC interface wave (node 3) extend not along this interface but rather through the TGO wave midline 
(node 5) followed by an extension into the top coat just above the YSZ/TGO interface wave's base (node 2), Figure 71.

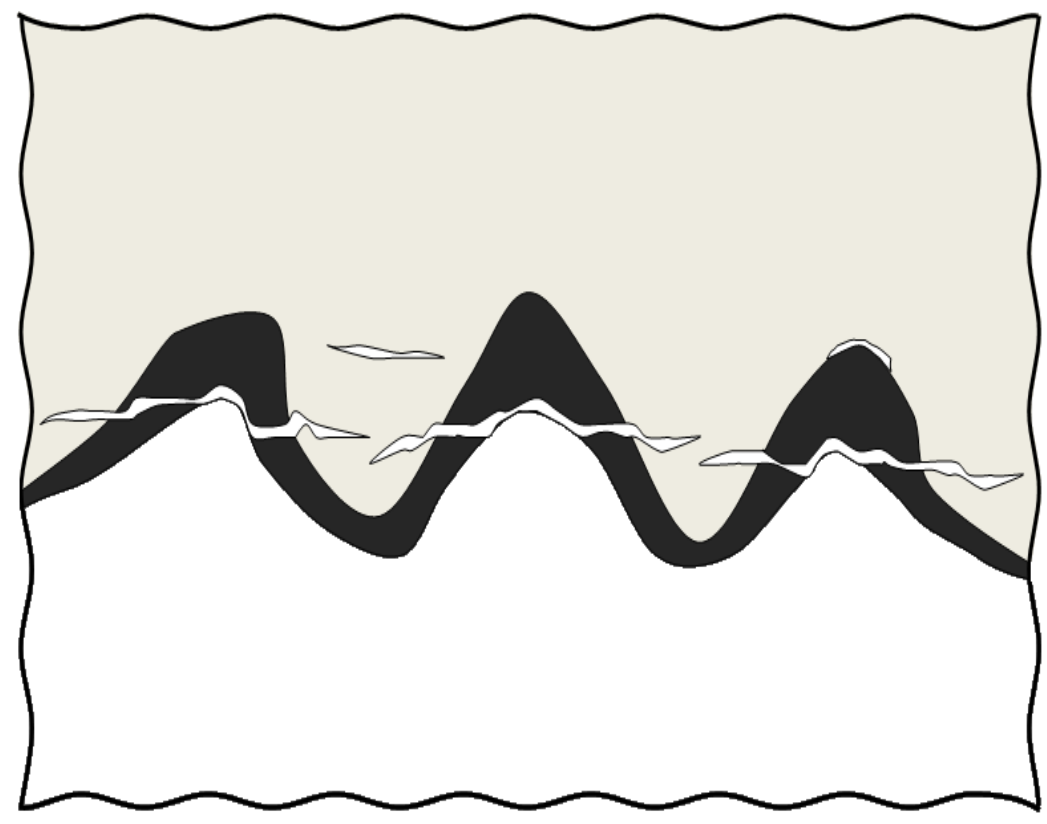

Figure 71: YSZ/YGO/BC interface failure pattern.

Although this failure pattern has been continually observed, its cause has been attributed to simply oxide scale thickness; however these results indicate that not only is it a function of TGO thickness but uniformity/geometry as well. In the event that oxide scales with a large $\beta$ develop, the out-of-plane residual stresses responsible for these failure patterns become present much earlier in the TBC's life cycle and ultimately lead to premature debonding. 


\section{Chapter 4.0: Conclusion}

Following a Hertzian contact mechanics approach, a load-based microindentation method has been developed for elastic modulus measurement of unknown materials. Utilizing an automated multiple-loading/unloading indentation testing procedure, this micro-indentation technique does not require contact area and/or indentation depth measurements alleviating much of the cost associated with prevailing nano- and micro-indentation evaluation systems. This technique has also led to the development of a portable, hand held load-depth sensing indentation system capable of accurately obtaining elastic modulus values of various metallic alloys with both flat and tubular surfaces. Furthermore, a probe for mechanical property evaluation of materials at elevated temperatures (to $1000^{\circ} \mathrm{C}$ ) has been developed as well. Calibration tests conducted on $\mathrm{H} 13$ Tool Steel from $22^{\circ} \mathrm{C}$ to $500^{\circ} \mathrm{C}$ and Haynes 230 at $1000^{\circ} \mathrm{C}$ generated results in excellent agreement with reported literature values. Additionally, preliminary creep tests of Haynes 230 alloy at $1200^{\circ} \mathrm{C}$ showed good correlation with reported creep exponents. Further system enhancements and additional testing under controlled environmental conditions are planned for the next-phase of this research effort. These evaluations will ultimately aid it the development of more sophisticated numerical models which predict spallation failure of TBC systems applied to the hot sections of land-based turbines.

Additionally, the unloading surface stiffness responses obtained from TBC coupons along thermal loadings has proven to be a viable analysis tool for the determination of spallation failure location. Using a multiple partial unloading procedure, slopes from various loads were able to be evaluated for abnormalities or trends along ever increasing thermal exposures at $1100^{\circ} \mathrm{C}$ in ambient air environments. Failure locations for both isothermal and cyclically exposed APS/MCrAIY/RenéN5 and EB-PVD/MDL/RenéN5 TBC coupons were correctly predicted with a $90 \pm 3 \mathrm{~N}$ evaluation load. Ultimately, residual stresses within the TGO and YSZ due to thermal expansion mismatches create subtle changes in measured stiffness response. These increases in unloading slope produce a 
numerical value from which initial spallation failure location is predicted. The development of this non-destructive evaluation technique is exclusive in that it is not only able to detect spallation site location but do so prior to its occurrence. Furthermore, experimentally observed micro-cracking and delamination patterns have been analyzed numerically along the geometrical parameter $\beta$. The results of this study indicate that the tensile out-of-plane residual stresses acting within the YSZ/TGO/BC interface and responsible for large scale spallation failure emerge prior to the development of sufficiently thick oxide scale as previously thought but rather as $\beta$ exceeds 1. Cross-sectional scanning electron micrographs of thermally cycled APS/MCrAIY/RenéN5 TBC coupons displaying TGO morphologies resembling that of a variable thickness sinusoidal wave were enlisted to develop a finite element TBC system model. The residual stress states at five locations within this model were evaluated as possible crack initiation sites along $\beta$, from this it was determined that large shears stress developing at the TGO wave midline allow cracks generated at the apex of the TGO/BC interface to extend to the YSZ just above the sinusoidal wave's base. Although TGO thickness has been shown capable of producing the out-of-plane tensile stresses required to initiate these failure patterns, variable thickness oxide scale geometries will produce such stresses significantly earlier in the TBC's life cycle and ultimately lead to premature failure.

\subsection{Recommendations}

A high temperature load-based micro-indentation method has been developed for elastic modulus measurement of unknown materials. Although accurate, some concerns still exist when applying the multiple partial unloading technique at ever increasing temperatures, where evaluation sample surface oxidation rates have the ability to greatly affect the rendered result. This however has been shown to have little to no effect if evaluation loads are increased proportionally to oxide scale thickness. To void this however, indentation testing above a material's oxidation temperature should be conducted under environmentally controlled conditions. Furthermore, as evaluation temperature 
increases, a furnaces ability to maintain a desired set point decreases and thus amplifies sample thermal drift. Caused by coefficients of thermal expansion, both reductions and amplifications in load are observed when the sample's temperature fluctuates. However this problem may be avoided by reducing required evaluation time as well. Ultimately the use of a multiple partial unloading algorithm allows indentation based material evaluation techniques to be easily applied despite the presence of harsh environmental conditions.

The evaluation of surface stiffness response obtained from TBC systems between thermal cyclic loadings has been shown capable of predicting the location at which initial spallation will occur. However, all coupons evaluated in this study were of a symmetric rectangular geometry. As a result, the locations of highest residual stress state occur at the TBC systems free edges and ultimately cause failure to initiate from this region. Thus to confirm the findings of this case study it would be advised to perform a similar experiment of TBC systems with either circular or cylindrical geometries. Additionally, due to their multilayered structures, TBC system surface stiffness responses are a combination of several degraded materials' mechanical properties. However, each material's mechanical properties may not be evaluated individually as the coefficients of thermal expansion mismatch within the TBC matrix produce tensile/compressive strains which prevent complete sintering of the YSZ topcoat under thermal loads. Finally, thermal cyclic degradation of TBC systems although comparable does not recreate the environment to which these coatings are exposed in industrial applications. The relationship and effects of degradation mechanisms such as erosion and particle bombardment on surface stiffness response have not been considered in this study.

Although continually observed TBC failure patterns have been described by various researchers, previously these mechanisms have been attributed to TGO thickness increases only. However current results indicate that it is both a function of TGO thickness and geometry as well. In the event that oxide scales with a large $\beta$ develop, the out-of-plane residual stresses responsible for these failure patterns will become present much earlier in the TBC's life cycle and 
ultimately lead to premature debonding. This effect must be considered while engineering and manufacturing TBC system bond coats. Additionally, although some level of surface roughness is required to mechanically bond APS topcoat structures to the alloy based bond coats; not only slow but also uniform oxidation rates across their surface should be generated. Consideration if these finding will lead to an extension of TBC life cycle fatigue limits.

\subsection{Final Remarks}

Contributions to TBC and indentation related research topics have been presented. In summary a micro-indentation technique for NDE testing of TBC system materials subjected to isothermal and cyclic loadings at $1100^{\circ} \mathrm{C}$ in air has shown to be a viable tool for initial spallation location prediction. Additionally, scanning electron micrographs of thermally loaded TBC systems displayed three distinctive oxide geometry growth trends. From this, several object oriented numerical models were developed along the defined oxide scale geometry parameter $\beta$. Results from this study indicate that although TGO thickness has previously been shown capable of producing the out-of-plane tensile stresses required to initiate failure, variable thickness oxide scale geometries with $\beta>1$

produce such stresses significantly earlier in the TBC's life cycle and ultimately lead to premature failure. Finally, following a classical Hertzian contact mechanics approach, a load-based multiple partial unloading micro-indentation technique which does not require high precision depth sensors has been used to develop a micro-indentation system capable of acquiring material mechanical properties at temperatures above $1000^{\circ} \mathrm{C}$. 


\section{References}

1. G. W. Goward, Progress in Coatings for Gas Turbine Airfoils, Surface and Coatings Technology, 108-109, 1-3 (1998).

2. P. E. Hodge, S. Stecura, M. A. Gedwill, I. Zaplatynsky, S. R. Levine, Thermal Barrier Coatings: Burner Rig Hot Corrosion Test Results, National Aeronautics and Space Administration, Technical Memorandum, 79005 (1978).

3. B. Gleeson, Thermal Barrier Coatings for Aeroengine Applications, Journal of Propulsion and Power, 22, 2 (2006).

4. U. Schulz, K. Fritscher, C. Leyens and M. Peters, Influence of Processing on Microstructure and Performance of Electron Beam Physical Vapor Deposition (EB-PVD) Thermal Barrier Coatings, Journal of Engineering for Gas Turbines and Power, 124, 2 (2002).

5. U. Schulz, C. Leyens, K. Fritscher, M. Peters, B. Saruhan-Brings, O. Lavigne, J.-M. Dorvaux, M. Poulain, R. Mévrel and M. Caliez, Some Recent Trends in Research and Technology of Advanced Thermal Barrier Coatings, Applied Science and Technology, 7, 1 (2003).

6. J. T. DeMasi-Marcin, K. D. Sheffler and S. Bose, Mechanisms of Degradation and Failure in a Plasma-Deposited Thermal Barrier Coating, Journal of Engineering for Gas Turbines and Power, 112, 4 (1990).

7. D. Naumenko, V. Shemet, L. Lorenz and W. F. Quadakkers, Failure Mechanisms of Thermal Barrier Coatings on MCrAlY-Type Bondcoats Associated with the Formation of the Thermally Grown Oxide, Journal of Materials Science, 44, 7 (2009).

8. R. Vaßen, G. Kerkhoff and D. Stöver, Development of a Micromechanical Life Prediction Model for Plasma Sprayed Thermal Barrier Coatings, Materials Science and Engineering: A, 303, 1-2 (2001).

9. A. C. Fisher-Cripps, Introduction to Contact Mechanics, Springer-Verlag, New York, New York (2000). 
10. K. L. Johnson, K. Kendall and A. D. Roberts, Surface Energy and Contact Mechanics of Elastic Solids, Proceedings of the Royal Society of London, 324, 1558 (1971).

11. H. Hertz, On the Contact of Elastic Solids. J. Reine Agnew. Math. 92, 1881, Translated and reprinted in English in Hertz's Miscellaneous Papers, Macmillan \& Company, London (1896).

12. A. I. Lure, Three-Dimensional Problems in the Theory of Elasticity, Gostekhizdat, edited by J. R. M. Radok, Interscience, New York (1964).

13. A. Bolshakov and G. M. Pharr, Influences of Pileup on the Measurement of Mechanical Properties by Load and Depth sensing Indentation Techniques, Journal of Materials Research, 13, 4 (1998).

14. W. C. Oliver and G. M. Pharr, An Improved Technique for Determining Hardness and Elastic Modulus Using Load and Displacement Sensing Indentation Experiments, Journal of Materials Research, 7, 6 (1992).

15. C. Feng, Development of a Transparent Indenter Measurement System and Indentation Analysis for Material Mechanical Property Evaluation, West Virginia University Libraries, Dissertation (2005).

16. C. Feng and B. S. Kang, A Simple Indentation Measurement for Surface Mechanical Property Evaluation, Materials Science and Technology 2007 Conference and Exhibition, Michigan (2007).

17. ASTM Standard, Standard Practice for Instrumented Indentation Testing, Designation: E2546-07, ASTM International, West Conshohocken, Pennsylvania (2007).

18. A. S. Kobayashi, Handbook on Experimental Mechanics, Second Revised Edition, Society of Experimental Mechanics, Bethel, Connecticut (1993).

19. A. C. Fisher-Cripps, Nanoindentation, Second Edition, Springer+Business Media, New York, New York (2004).

20. J. M. Tannenbaum, The Development of an Instrumented Indentation System, West Virginia University Libraries, Thesis (2008). 
21. G. Feng and A. H. W. Ngan, Effects of Creep and Thermal Drift on Modulus Measurement using Depth-Sensing Indentation, Journal of Materials Research, 17, 3 (2002).

22. J. H. Underwood, Residual-Stress Measurement using Surface Displacements around an Indentation, Experimental Mechanics, 13, 9 (1973).

23. T. Y. Tsui, W. C. Oliver and G. M. Pharr, Influences of Stress on the Measurement of Mechanical Properties Using Nanoindentation: Part I. Experimental in an Aluminum Alloy, Journal of Materials Research, 11, 3 (1996).

24. S. Carlsson and P.-L. Larsson, On the Determination of Residual Stress and Strain Fields by Sharp Indentation Testing. Part II: Experimental Investigation, Acta Materialia, 49, 12 (2001).

25. A. Bolshakov, W. C. Oliver and G. M. Pharr, Influences of Stress on the Measurement of Mechanical Properties Using Nanoindentation: Part II. Finite Element Simulations, Journal of Materials Research, 11, 3 (1996).

26. J. G. Swadener, B. Taljat and G. M. Pharr, Measurement of Residual Stresses by Load and Depth Sensing Spherical Indentation, Journal of Materials Research, 16, 7 (2001).

27. X. Chen, J. Yan and A. M. Karlsson, On the Determination of Residual Stress and Mechanical Properties by Indentation, Material Science and Engineering: A, 416, 1-2 (2006).

28. B. Taljat and G. M. Pharr, Measurement of Residual Stresses by Load and Depth Sensing Spherical Indentation, Materials Research Society Symposium Proceedings, 594 (2000).

29. S. Carlsson and P.-L. Larsson, On the Determination of Residual Stress and Strain Fields by Sharp Indentation Testing. Part I: Theoretical and Numerical Analysis, Acta Materialia, 49, 12 (2001).

30. S. Suresh and A. E. Giannakopoulos, A New Method for Estimating Residuals Stresses by Instrumented Sharp Indentation, Acta Materialia, 46, 16 (1998). 
31. K. Kese and D. J. Rowcliffe, Nanoindentation Method for Measuring Residual Stress in Brittle Materials, Journal of the American Ceramic Society, 86, 5 (2003).

32. Å. K. Jämting, J .M . Bell, M. V. Swain and N. Schwarzer, Investigation of the Elastic Modulus of Thin Films using Simple Biaxial Bending Techniques, Thin Solid Films, 308-309 (1997).

33. K. L. Johnson, Contact Mechanics, Cambridge University Press, New York, New York (1985).

34. G. Feng and A. H. W. Ngan, Effects of Creep and Thermal Drift on Modulus Measurement using Depth-Sensing Indentation, Journal of Materials Research, 17, 3 (2002).

35. D. Dorner amd B. Stöckhert, Plastic Flow Strength of Jadeite and Diopside Investigated by Microindentation Hardness Tests, Tectonophysics, 379, 14 (2004).

36. B. D. Beakea and J. F. Smith, High-Temperature Nanoindentation Testing of Fused Silica and Other Materials, Philosophical Magazine A, 82, 10 (2002).

37. M. Watanabe, C. Mercer, C. G. Levi and A. G. Evans, A Probe for the High Temperature Deformation of Thermal Barrier Oxides, Acta Materialia, 52, 6 (2004).

38. B. Passilly, P. Kanoute, F.-H. Leroy and R. Mévrel, High Temperature Instrumented Microindentation: Applications to Thermal Barrier Coating Constituent Materials, Philosophical Magazine, 86, 33-35 (2006).

39. C. A. Schuh, C. E. Packard and A. C. Lund, Nanoindentation and ContactMode Imaging at High Temperatures, Journal of Materials Research, 21, 3 (2006).

40. C. Feng, J. M. Tannenbaum, B. S. Kang, and M. A. Alvin, A Load-Based Multiple-Partial Unloading Micro-Indentation Technique for Mechanical Property Evaluation, Society of Experimental Mechanics, Springer US (2009). 
41. J. B. Wachtman, W. E. Tefft, D. G. Lam, and C. S. Apstein, Exponential Temperature Dependence of Young's Modulus for Several Oxides, Physical Review , 122, 6 (1961).

42. R. G. Munro, Evaluated Material Properties for a Sintered $\alpha$-Alumina, Journal of the American Ceramic Society, 80, 8 (1997).

43. M. F. Rothman, High Temperature Property Data: Ferrous Alloys, ASM International, Metals Park, Ohio (1988).

44. J. M. Tannenbaum, B. J. Conklin, B. S.-J. Kang, and M. A. Alvin, Development of a High Temperature Micro-Indentation Technique for Material Mechanical Property Evaluation up to $1200^{\circ} \mathrm{C}$, TMS Annual Meeting and Exhibition, February 14-18, Seattle, WA (2010).

45. X. Deng, N. Chawla, K. K. Chawla, M. Koopman and J. P. Chu, Mechanical Behavior of Multilayered Nanoscale Metal-Ceramic Composites, Advanced Engineering Materials, 7, 12 (2005).

46. J. M. Tannenbaum, K. Lee, B. S.-J. Kang and M. A. Alvin, Non-Destructive Spallation Prediction of Thermal Barrier Coatings by a Load-Based MicroIndentation Technique, MS\&T Annual Conference \& Exhibition, October 17-21, Houston, Texas (2010).

47. S. M. Meier and D. K. Gupta, The Evolution of Thermal Barrier Coatings in Gas Turbine Engine Applications, Journal of Engineering for Gas Turbines and Power, 116, 1 (1994).

48. J. Chen and B. Young, Stress-Strain Curves for Stainless Steels at Elevated Temperatures, Engineering Structures, 28, 2 (2006).

49. J. P. Singh, B. Nair, D. Renusch, M. Sutaria and M. Grimsditch, Damage Evolution and Residual Stresses in Plasma-Sprayed Zirconia Thermal Barrier Coatings, Ceramic Engineering and Science Proceedings, 20, 3 (1999).

50. J. P. Singh, B. G. Nair, D P. Renusch, M. P. Sutaria and M. H. Grimsditch, Damage Evolution and Stress Analysis in Zirconia Thermal Barrier Coatings during Cyclic and Isothermal Oxidation, Journal of the American Ceramic Society, 84, 10 (2001). 
51. A. Radiei and A.G. Evans, Failure Mechanisms Associated with the Thermally Grown Oxide in Plasma-Sprayed Thermal Barrier Coatings, Acta Materialia, 48, 15 (2000).

52. L. Lelait, S. Alpérine, and R. Mévrel, Alumina Scale Growth at ZirconiaMCrAlY Interfaces: A Microstructural Study, Journal of Materials Science, 27, 1 (1992).

53. H. G. Scott, Phase Relationships in Zirconia-Yttria Systems, Journal of Materials Science, 10, 8 (1975).

54. S. Bose, High Temperature Coatings, Butterworth-Heinemann, Elsevier Incorporated, Oxford, United Kingdom (2007).

55. S. Stecura, Effects of Compositional Changes on the Performance of a Thermal Barrier Coating System, National Aeronautics and Space Administration, Technical Memorandum, 78976 (1978).

56. S. Bose and J DeMasi-Marcin, Thermal Barrier Coating Experience in Gas Turbine Engines at Pratt \& Whitney, Journal of Thermal Spray Technology, 6, 1 (1997).

57. W. Beele, G. Marijnissen and A. van Lieshout, The Evolution of Thermal Barrier Coatings - Status and Upcoming Solutions for Today's Key Issues, Surface and Coatings Technology, 120-121 (1999).

58. D. Pan, M. W. Chen, P. K. Wright and K. J. Hemker, Evolution of a Diffusion Aluminide Bond Coat for Thermal Barrier Coatings During Thermal Cycling, Acta Materialia, 51, 8 (2003).

59. D. Stöver and C. Funke, Directions of the Development of Thermal Barrier Coatings in Energy Applications, Journal of Materials Processing Technology, 92-93 (1999).

60. N. Czech, F. Schmitz and W. Stamm, Microstructural Analysis of the Role of Rhenium in Advanced MCrAlY Coatings, Surface and Coatings Technology, 76-77, 1 (1995).

61. T. A. Taylor and D. F. Bettridge, Development of Alloyed and DispersionStrengthened MCrAlY Coatings, Surface and Coatings Technology, 86-87, 1 (1996). 
62. M. Subanovic, D. Sebold, R. Vassen, E. Wessel, D. Naumenko, L. Singheiser and W. J. Quadakkers, Effect of Manufacturing Related Parameters on Oxidation Properties of MCrAlY-Bondcoats, Materials and Corrosion, 59, 6 (2008).

63. E. P. Busso and F. A. McClintock, Thermal Fatigue Degradation of an Overlay Coating, Materials Science and Engineering: A, 161, 2 (1993).

64. V. K. Tolpygo and D. R. Clarke, Microstructural Evidence for CounterDiffusion of Aluminum and Oxygen During the Growth of Alumina Scales, Proceedings of $5^{\text {th }}$ International Conference on the Microscopy of Oxidation, Republic of Ireland, August 26-28 (2002).

65. M. Hu, S. Guo, T. Tomimatsu, Y. Ikuhara and Y. Kagawa, TEM Study on Microstructure of Thermally Grown Oxide in EB-PVD Thermal Barrier Coatings, Surface and Coatings Technology, 200 (2006).

66. V. Teixeira, Mechanical Integrity in PVD Coatings due to the Presence of Residual Stresses, Thin Film Solids, 392, 2 (2001).

67. A. G. Evans, D. R. Clarke and C. G. Levi, The Influence of Oxides on the Performance of Advanced Gas Turbine, Journal of the European Ceramic Society, 28, 7 (2008).

68. H. Echsler, D. Renusch and M. Schütze, Bond Coat Oxidation and its Significance to for Life Expectancy of Thermal barrier Coating Systems, Materials Science and Technology, 20, 3 (2004).

69. D. Naumenko, B. Gleeson, E. Wessel, L. Singheiser and W.J. Quadakkers, Correlation between the Microstructure, Growth Mechanism, and Growth Kinetics of Alumina Scales on a FeCrAlY Alloy, Metallurgical and Materials Transactions A, 38, 12 (2007).

70. E. Wessel, V. Kochubey, D. Naumenko, L. Niewolak, L. Singheiser and W. J. Quadakkers, Effects of Zr Addition on the Microstructure of the Alumina Scales on FeCrAly-Alloys, Scripta Materialia, 51, 10 (2004).

71. C. Mercer, S. Faulhaber, N. Yao, K. Mcllwrath and O. Fabrichnaya, Investigation of the Chemical Composition of the Thermally Grown Oxide 
Layer in Thermal Barrier Systems with NiCoCrAlY Bond Coats, Surface and Coatings Technology, 201, 3-4 (2006).

72. C. T. Sims and W. C. Hagel, The Superalloys, John Wiley \& Sons, New York (1972).

73. P. Caron and T. Khan, Evolution of Ni-based Superalloys for Single Crystal Gas Turbine Blade Applications, Aerospace Science and Technology, 3 , 8 (1999).

74. U. Schulz, S. G. Terry and C. G. Levi, Microstructure and Texture of EBPVD TBCs Grown Under Different Rotation Modes, Materials Science and Engineering: A, 360, 1-2 (2003).

75. S. Sampath and H. Herman, Rapid Solidification and Microstructure Development during Plasma Spray Deposition, Journal of Thermal Spray Technology, 5, 4 (1996).

76. J. Madejski, Solidification of Droplets on a Cold Surface, International Journal of Heat and Mass Transfer, 19, 9 (1976).

77. S. Sampath, X. Y. Jiang, J. Matejicek, A. C. Leger and A. Vardelle, Substrate Temperature Effects on Splat Formation, Microstructure Development and Properties of Plasma Sprayed Coatings Part I : Case Study for Partially Stabilized Zirconia, Materials Science and Engineering: A, 272, 1 (1999).

78. E. P. Busso, L. Wright, H. E. Evans, L. N. McCartney, S. R. J. Saunders, S. Osgerby and J Nunn, A Physics-Based Life Prediction Methodology for Thermal Barrier Coating Systems, Acta Materialia, 55, 5 (2007).

79. P. Niranatlumpong, C. B. Ponton and H. E. Evans, The Failure of Protective Oxides on Plasma-Sprayed NiCrAlY Overlay Coatings, Oxidation of Metals, 53, 3-4 (2000).

80. J. T. DeMasi, K. D. Sheffler and M. Ortiz, Thermal Barrier Coating Life Predication Model Development: Phase I-Final Report, National Aeronautics and Space Administration, Contractor Report, 182230 (1989). 
81. S. M. Meier, D. M. Nissley and K. D. Sheffler, Thermal Barrier Coating Life Predication Model Development: Phase II-Final Report, National Aeronautics and Space Administration, Contractor Report, 189111 (1991).

82. H. E. Evans, A. Strawbridge, R. A. Carolan and C.B. Ponton, Creep Effects on the Spallation of an Alumina Layer from a NiCrA/Y Coating, Materials Science and Engineering: A, 225, 1 (1997).

83. T. S. Hille, T. J. Nijdam, A. S. J. Suiker, S. Turteltaub and W. G. Sloof, Damage Growth Triggered by Interface Irregularities in Thermal Barrier Coatings, Acta Materialia, 57, 9 (2009).

84. K. Vaidyanathana, M. Gell and E. Jordan, Mechanisms of Spallation of Electron Beam Physical Vapor Deposited Thermal Barrier Coatings with and Without Platinum Aluminide Bond Coat Ridges, Surface and Coating Technology, 133-134 (2000).

85. R. Janakiraman, G. H. Meier and F. S. Pettit, The Effect of Water Vapor on the Oxidation of Alloys that Develop Alumina Scales for Protection, Metallurgical and Materials Transactions, 33, 11 (1999).

86. A. G. Evans, D. R. Mumm, J. W. Hutchinson, G. H. Meir and F. S. Pettit, Mechanisms Controlling the Durability of Thermal Barrier Coatings, Progress in Materials Science, 46, 5 (2001).

87. U. Schulz, M. Menzebach, C. Leyensa and Y.Q. Yang, Influences of Substrate Material on Oxidation Behavior and Cyclic Lifetime of EB-PVD TBC Systems, Surface and Coatings Technology, 146-147, 5-6 (2001).

88. T. J. Nijdam, G. H. Marijnissen, E. Vergeldt, A. B. Kloosterman and W. G. Sloof, Development of a Pre-Oxidation Treatment to Improve the Adhesion between Thermal Barrier Coatings and NiCoCrA/Y Bond Coatings, Oxidation of Metals, 66, 5-6 (2006).

89. M. Subanovic, D. Sebold, R. Vassen, E. Wessel, D. Naumenko, L. Singheiser and W. J. Quadakkers, Effect of Manufacturing Related Parameters on Oxidation Properties of MCrAlY-Bondcoats, Materials and Corrosion, 59, 6 (2008). 
90. P. K. Wright and A. G. Evans, Mechanisms Governing the Performance of Thermal Barrier Coatings, Current Opinions in Solid State and Materials Science, 4, 3 (1999).

91. S. R. Choi, J. W. Hutchinson, A. G. Evans, Delamination of Multilayer Thermal Barrier Coatings, Mechanics of Materials, 31, 7 (1999).

92. H. E. Echsler, V. Shemet, M. Schutze, L. Singheiser and W. J. Quadakkers, Cracking In and Around the Thermally Grown Oxide in Thermal Barrier Coatings: A Comparison of Isothermal and Cyclic Oxidation, Journal of Materials Science, 41, 4 (2006).

93. N. P. Padture, M. Gell and E. H. Jordan, Thermal Barrier Coatings for Gas-Turbine Engine Applications, Science, 296, 5566 (2002).

94. M. Martena, D. Botto, P. Fino, S. Sabbadini, M. M. Gola and C. Badini, Modeling of TBC System Failure: Stress Distribution as a Function of TGO Thickness and Thermal Expansion Mismatch, Engineering Failure Analysis, 13, 3 (2006).

95. A. G. Evans, G. B. Crumley and R. E. Demaray, On the Mechanical Behavior of Brittle Coatings and Layers, Oxidation of Metals, 20, 5-6 (1983).

96. C. H. Hsueh, J. A. Haynes, M. J. Lance, P. F. Becher, M. K. Ferber, E. R. Fuller, S. A. Langer, W. C. Carter and W. R. Cannon, Effects of Interface Roughness on Residual Stresses in Thermal Barrier Coatings, Journal of the American Ceramic Society, 82, 4 (1999).

97. J. A. Haynes, M. K. Ferber, W. D. Porter, and E. D. Rigney, Characterization of Alumina Scales Formed During Isothermal and Cyclic Oxidation of Plasma-Sprayed TBC Systems at $1150^{\circ} \mathrm{C}$, Oxidation of Metals, 52, 1-2 (1999).

98. P. Bednarz, Finite Element Simulation of Stress Evolution in Thermal Barrier Coating Systems, Dissertation, Schriften des Forschungszentrums Jülich, Energy Technology (2007). 
99. F. Tang and J. M. Schoenung, Local Accumulation of Thermally Grown Oxide in Plasma-Sprayed Thermal Barrier Coatings with Rough TopCoat/Bond-Coat Interfaces, Scripta Materialia, 52, 9 (2005).

100. P. Bednarz, R. Herzog, E. Trunova, R. W. Steinbrech, H. Eschler, W. J. Quaddakers, F. Schubert and L. Singheiser, Stress Distribution in APSTBCs Under Thermal Cycling Loading Conditions, 29th International Conference on Advanced Ceramics and Composites, January 23-28, Cocoa Beach, Florida (2005).

101. P. Bednarz, Finite Element Simulation of Stress Evolution in Thermal Barrier Coating Systems, Dissertation, Schriften des Forschungszentrums Jülich, Energy Technology (2007).

102. O. Trunova, T. Beck. R. Herzog, R. W. Steinbrech and L. Singheiser, Damage Mechanisms and Lifetime Behavior of Plasma Sprayed Thermal Barrier Coating Systems for Gas Turbines - Part I: Experiments, Surface Coatings and Technology, 202, 20 (2008).

103. T. Beck. R. Herzog, O. Trunova, M. Offermann, R. W. Steinbrech and L. Singheiser, Damage Mechanisms and Lifetime Behavior of Plasma Sprayed Thermal Barrier Coating Systems for Gas Turbines - Part II: Modeling, Surface Coatings and Technology, 202, 24 (2008).

104. K. W. Schlichting, N. P. Padture, E. H. Jordan and M. Gell, Failure Modes in Plasma-Sprayed Thermal Barrier Coatings, Materials Science and Engineering: A, 342, 1-2 (2003).

105. K. W. Schlichting, K. Vaidyanathan, Y. H. Sohn, E. H. Jordan, M. Gell and N. P. Padture, Application of $\mathrm{Cr}^{3+}$ Photoluminescence Piezo-Spectroscopy to Plasma-Sprayed Thermal Barrier Coatings for Residual Stress Measurement, Materials Science and Engineering: A, 291, 1-2 (2000).

106. A. Portinha, V. Teixeria, J. Carneiro, M. G. Beghi, C. E. Bottani, N. Franco, R. Vassen, D. Stoever and A. D. Sequeira, Residual Stresses and Elastic Modulus of Thermal Barrier Coatings Graded in Porosity, Surface and Coatings Technology, 188-189 (2004). 
107. D. W. Jordan and K. T. Faber, X-Ray Residual Stress Analysis of a Ceramic Thermal Barrier Coating Undergoing Thermal Cycling, Thin Film Solids, 235, 1-2 (1993).

108. T. W. Clyne and S. C. Gill, Residual Stresses in Thermal Spray Coatings and Their Effect on Interfacial Adhesion: A Review of Recent Work, Journal of Thermal Spray Technology, 5, 4 (1996).

109. L. Fu, K. A. Khor, H. W. Ng and T. N. Teo, Non-Destructive Evaluation of Plasma Sprayed Functionally Graded Thermal Barrier Coatings, Surface and Coatings Technology, 130 (2000).

110. H. K. Sezer, L Li, Z. Wu, B. Anderson and P. Williams, Non-Destructive Microwave Evaluation of TBC Delamination Induced by Acute Angle Laser Drilling, Measurement Science and Technology, 18 (2007).

111. G. Chen, K. N. Lee and S. N. Tewari, The Concept and Development of Emission Spectroscopy for the Non-Destructive Evaluation (NDE) of the Failure of Thermal Barrier Coatings, Non Destructive Testing and Evaluation, 40 (2007).

112. Y. C. Zhou and T. Hashida, Thermal Fatigue Failure Induced by Delamination in Thermal Barrier Coatings, International Journal of Fatigue, 24 (2002).

113. P. Crowther, Non Destructive Evaluation of Coatings for Land Based Gas Turbines using a Multi-Frequency Eddy Current Technique, British Institute of Non-Destructive Testing, 46, 9 (2004).

114. S. Aithal, G. Rousset, L. Bertrand, P. Cielo and S. Dallaire, Photoacoustic Characterization of Subsurface Defects in Plasma-Sprayed Coatings, Thin Solid Films, 119, 2 (1984).

115. D. H. LeMieux, On-Line Thermal Barrier Coating Monitoring for Real-Time Failure Protection and Life Maximization, United States Department of Energy, Final Technical Report (2005).

116. J. I. Eldridge, T. J. Bencic, C. M. Spuckler, J. Singh and D. E. Wolfe, Delamination-Indicating Thermal Barrier Coatings Using YSZ:EU Sublayers, Journal of the American Ceramic Society, 89, 10 (2006). 
117. J. I. Eldridge, J. Singh and D. E. Wolfe, Erosion-Indicating Thermal Barrier Coatings Using Luminescent Sublayers, Journal of the American Ceramic Society, 89, 10 (2006).

118. J. I. Eldridge, C. M. Spuckler and R. Martin, Monitoring Delamination Progression in Thermal Barrier Coatings by Mid-Infrared Reflectance Imaging, International Journal of Applied Ceramic Technology, 3, 2 (2006).

119. J. I. Eldridge, C. M. Spuckler and J. R. Markham, Determination of Scattering and Absorption Coefficients for Plasma-Sprayed YttriaStabilized Zirconia Thermal barrier Coatings at Elevated Temperatures, Journal of the American Ceramic Society, 92, 10 (2009).

120. Q. Ma and D. R. Clarke, Piezospectroscopic Determination of Residual Stresses in Polycrystalline Alumina, American Ceramic Society, 77, 2 (1994).

121. J. R. Christensen, D. M. Lipkin, D. R. Clarke and K. Murphy, Nondestructive Evaluation of the Oxidation Stresses Through Thermal Barrier Coatings using $\mathrm{Cr}^{3+}$ Piezospectroscopy, Applied Physics Letters, 69, 24 (1996).

122. Y. H. Sohn, K. Vaidyanthan, M. Ronski, E. H. Jordon and M. Gell, Thermal Cycling of EB-PVD/MCrA/Y Thermal Barrier Catings: II. Evolution of Photo-Stimulated Luminescence, Surface and Coating Technology, 146 (2001).

123. V. K. Tolpygo and D. R. Clarke, Morphological Evolution of Thermal Barrier Coatings Induced by Cyclic Oxidation, Surface and Coatings Technology, 163, 30 (2003).

124. J. He and D. R. Clarke, Determination of the Piezospectroscopic Coefficients for Chromium-Doped Sapphire, American Ceramic Society, 78, 5 (1995).

125. M. Wen, E. H. Jordan and M. Gell, Evolution of Photo-Stimulated Luminescence of EB-PVD/(Ni, Pt)Al Thermal Barrier Coatings, Materials Science and Engineering: A, 398, 1-2 (2005). 
126. B. Heeg and D. R. Clarke, Non-Destructive Thermal Barrier Coating (TBC) Damage Assessment using Laser-Induced Luminescence and Infrared Radiometry, Surface and Coatings Technology, 200, 5 (2005).

127. J. R. MacDonald, Impedance Spectroscopy, Annals of Biomedical Engineering, 20, 3 (1992).

128. S.-H. Song, P. Xiao and L.-Q. Weng, Evaluation of Microstructural Evolution in Thermal Barrier Coatings during Thermal Cycling Using Impedance Spectroscopy, European Ceramic Society, 25, 7 (2005).

129. K. Ogawa, D. Minkov, T. Shoji, M. Sato and H. Hashimoto, NDE of Degradation of Thermal Barrier Coatings by Means of Impendence Spectroscopy, Non Destructive Testing and Evaluation, 32, 3 (1999).

130. J. W. Byeon, B. Jayaraj, S. Vishweswaraiah, S. Rhee, V. H. Desai and Y. $\mathrm{H}$. Sohn, Non-Destructive Evaluation of Degradation in Multi-Layered Thermal Barrier Coatings by Electro Chemical Impedance Spectroscopy, Materials Science and Engineering: A, 407, 1-2 (2005).

131. F. Yang and P. Xiao, Nondestructive Evaluation of Thermal Barrier Coatings Using Impedance Spectroscopy, International Journal of Applied Ceramic Technology, 6, 3 (2009).

132. C. C. Berndt, Acoustic Emission Evaluation of Plasma-Sprayed Thermal Barrier Coatings, Journal of Engineering for Gas Turbines and Power, 107, 1 (1985).

133. J. Voyer, F. Gitzhofer and M. I. Boulos, Study of the Performance of TBC under Thermal Cycling Conditions using an Acoustic Emission Rig, Journal of Thermal Spray Technology, 7, 2 (1998).

134. A. C. Bento and D. P. Almond, The Accuracy of Thermal Wave Interferometry for the Evaluation of Thermophysical Properties of PlasmaSprayed Coatings, Measurement Science and Technology, 6,7 (1995).

135. S. K. Lau, D. P. Almond and P. M. Pate, Transient Thermal wave Techniques for the Evaluation of Surface Coatings, Journal of Physics D: Applied Physics, 24, 3 (1991). 
136. B. S.-J. Kang, C. Feng, J. M. Tannenbaum and M. A. Alvin, A Load-based Depth-sensing Indentation Technique for Damage Assessment of Thermal Barrier Coatings, Proceeding of ASME Turbo Expo 2009, Orlando, FL, June 8-9 (2009).

137. M. F. Triola, Elementary Statistics 4th Edition, Benjamin/Cummings Publishing, Redwood City, California (1989).

138. W. Mendenhall, Introduction to Probability and Statistics 5th Edition, Duxbury Press, North Scituate, Massachusetts (1979).

139. C. Rinaldi, L Maria and A. Corno, Use of Photo-Stimulated Luminescence Piezospectroscopy and Lifing Models to Evidence Film Cooling Effect on EB-PVD Ceramic Thermal Barriers of Ex-Service Blades, Proceedings of ASME Turbo Expo, June 14-18, Glasgow, UK (2010).

140. J. W. Dally and W. F. Riley, Experimental Stress Analysis, Third Edition, McGraw-Hill, New York (1991).

141. J. L. Smialek, D. Zhu and M. D. Cuy, Moisture-Induced Delamination Video of an Oxidized Thermal Barrier Coating, Scripta Materialia, 59, 1 (2008).

142. J. L. Smialek, Enigmatic Moisture Effects on $\mathrm{Al}_{2} \mathrm{O}_{3}$ Scale and TBC Adhesion, National Aeronautics and Space Administration, Technical Memorandum, 215206 (2008).

143. S. M. Hu, Film-Edge-Induced Stress Substrates, Journal of Applied Physics, 50, 7 (1979).

144. E. Suhir, An Approximate Analysis of Stresses in Multilayered Elastic Thin Films, Journal of Applied Mechanics, 55, 1 (1988).

145. M. Andritschky and V. Teixeira, Residual Stress and Adhesion of Molybdenum Coatings Produced by Magnetron Sputtering, Vacuum, 43, 5-7 (1992).

146. V. Teixeira, Mechanical Integrity in PVD Coatings due to the Presence of Residual Stresses, Thin Film Solids, 392, 2 (2001). 
147. J. Rösler, M. Bäker and K. Aufzug, A Parametric Study of the Stress State of Thermal Barrier Coatings, Part I: Creep Relaxation, Acta Materialia, 52, 16 (2004).

148. J. Rösler, M. Bäker and M. Volgmann, Stress State and Failure Mechanisms of Thermal Barrier Coatings: Role of Creep in Thermally Grown Oxide, Acta Materialia, 49, 18 (2001).

149. E. Tzimas, H. Mullejans, S. D. Peteves, J. Bressers and W. Stamm, Failure of Thermal Barrier Coating Systems Under Cyclic Thermomechanical Loading, Acta Materialia, 48, 18-19 (2000).

150. D. S. Balint and J. W. Hutchinson, An Analytical Model of Rumpling in Thermal Barrier Coatings, Journal of the Mechanics and Physics of Solids, 53, 4 (2005).

151. C. -H. Hsueh, J. A. Haynes, M. J. Lance, P. F. Becher, M. K. Ferber, E. R. Fuller, S. A. Langer, W. C. Carter and W. R. Cannon, Effects of Interface Roughness on Residual Stresses in Thermal Barrier Coatings, Journal of the American Ceramic Society, 82, 4 (1999).

152. R. Vaßen, G. Kerkhoff and D. Stöver, Development of a Micromechanical Life Prediction Model for Plasma Sprayed Thermal Barrier Coatings, Material Science and Engineering: A, 303, 1-2 (2001).

153. W.-F. Chen and D.-J. Han, Plasticity for Structural Engineers, SpringerVerlag, New York, New York (1988). 


\section{Appendix}

Presented in this section are detailed depictions of contour color maps generated for spallation location prediction of thermal loaded TBC systems. Unloading slopes obtained from each TBC coupon are presented using a contour color mapping scheme presented in an earlier section. The TBC coupons used in this study consisted of a $1 / 8$ " thick by 1 " square nickel-based single crystal RenéN5 substrate with an applied $300 \mu \mathrm{m}$ MCrAlY bond coat and an air plasma sprayed (APS) or electron beam physical vapor deposited (EB-PVD) $350 \mu \mathrm{m}$ yttria stabilized zirconia (YSZ) layer. Seven APS coupons were evaluated, four isothermally (Coupons $\mathrm{A}, \mathrm{B}, \mathrm{C}$ and $\mathrm{D}$ ) and three cyclically exposed to temperatures of $1100^{\circ} \mathrm{C}$ in air (Coupons $E, F$ and G). One EB-PVD TBC (Coupon $\mathrm{H})$ system was evaluated in this study and was thermal cyclically exposed. The contour color maps provide a visualization of variable and increasing surface stiffness for predicting TBC spallation failure locations. In the following more detailed contour color maps are shown for isothermally cycled coupons $A, B, C$, D, E, F, G and H. 

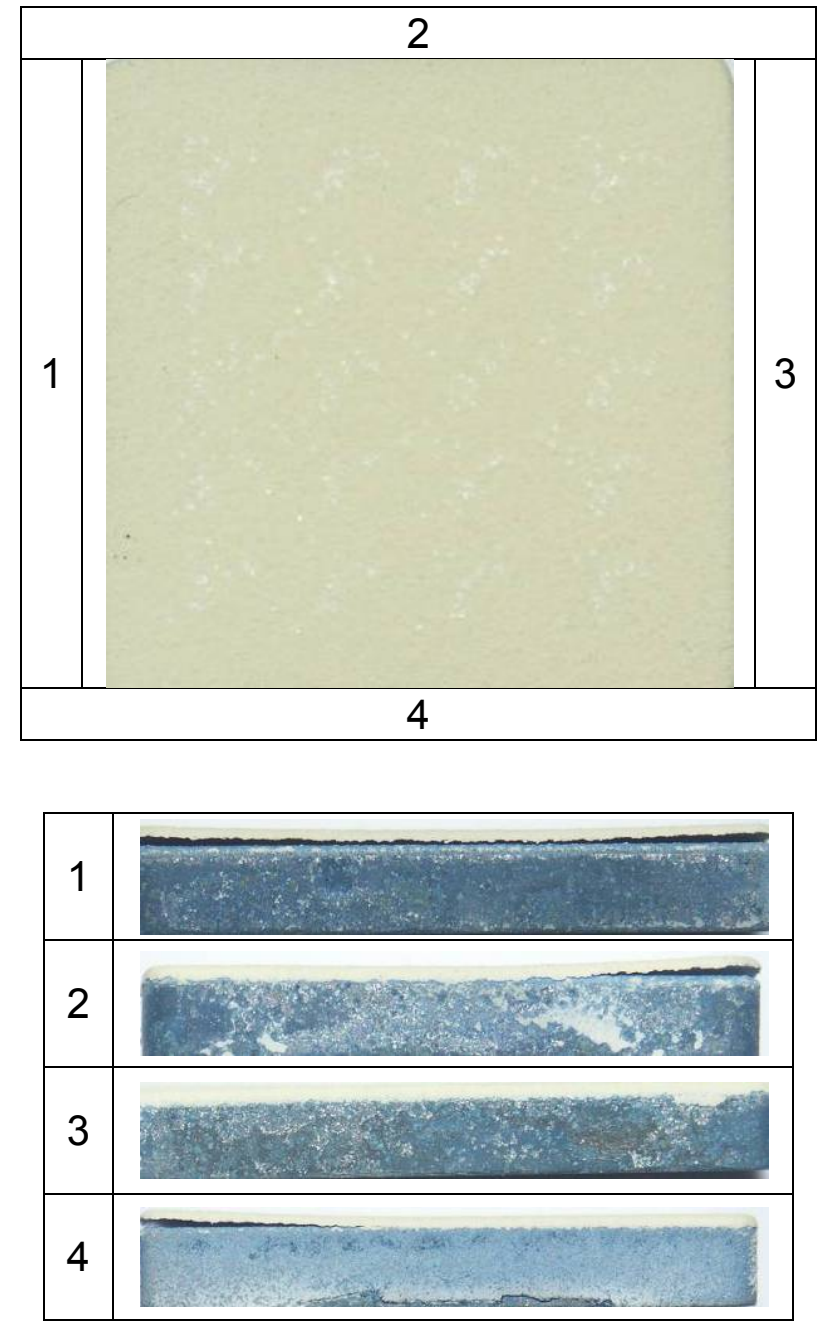

Figure 72: Edge and surface photographs of APS/MCrAIY/RenéN5 coupon A after a cumulative 500 isothermal heat treatment hours at $1100^{\circ} \mathrm{C}$ in an ambient air environment after 2 weeks storage.

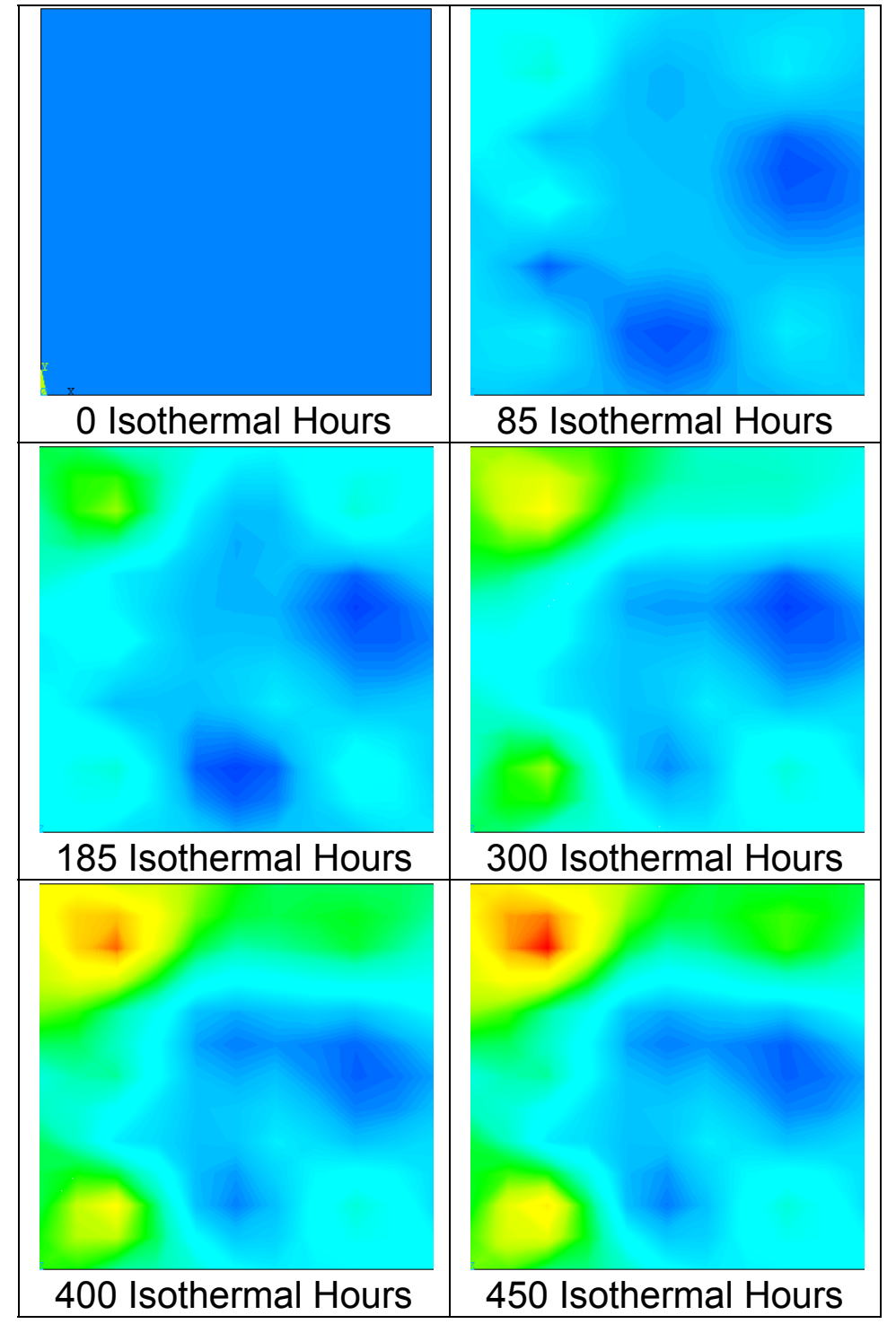

\section{Low Stiffness High Stiffness}

Figure 73: Surface stiffness response color maps for Coupon A obtained after each isothermal heat treatment. 

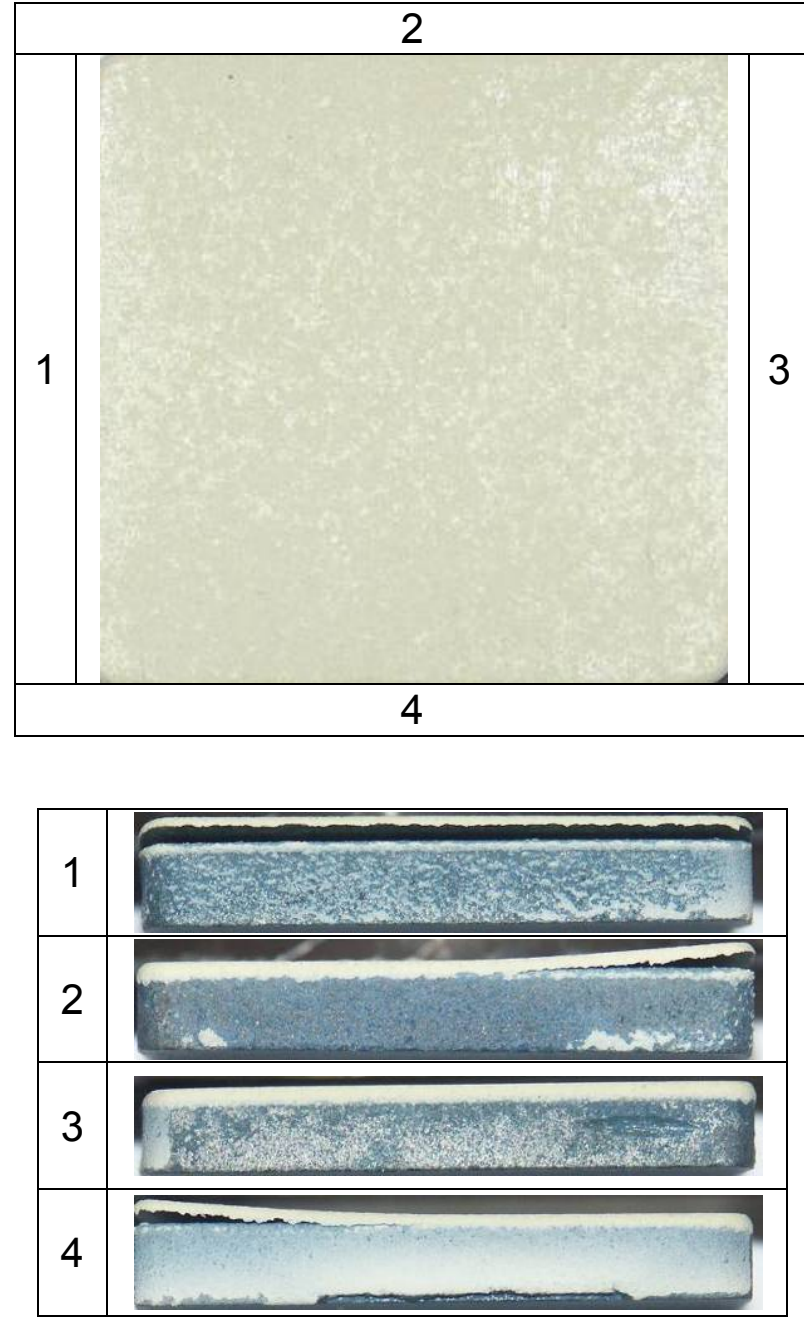

Figure 74: Edge and surface photographs of APS/MCrAIY/RenéN5 Coupon B after a cumulative 400 isothermal heat treatment hours at $1100^{\circ} \mathrm{C}$ in an ambient air environment.

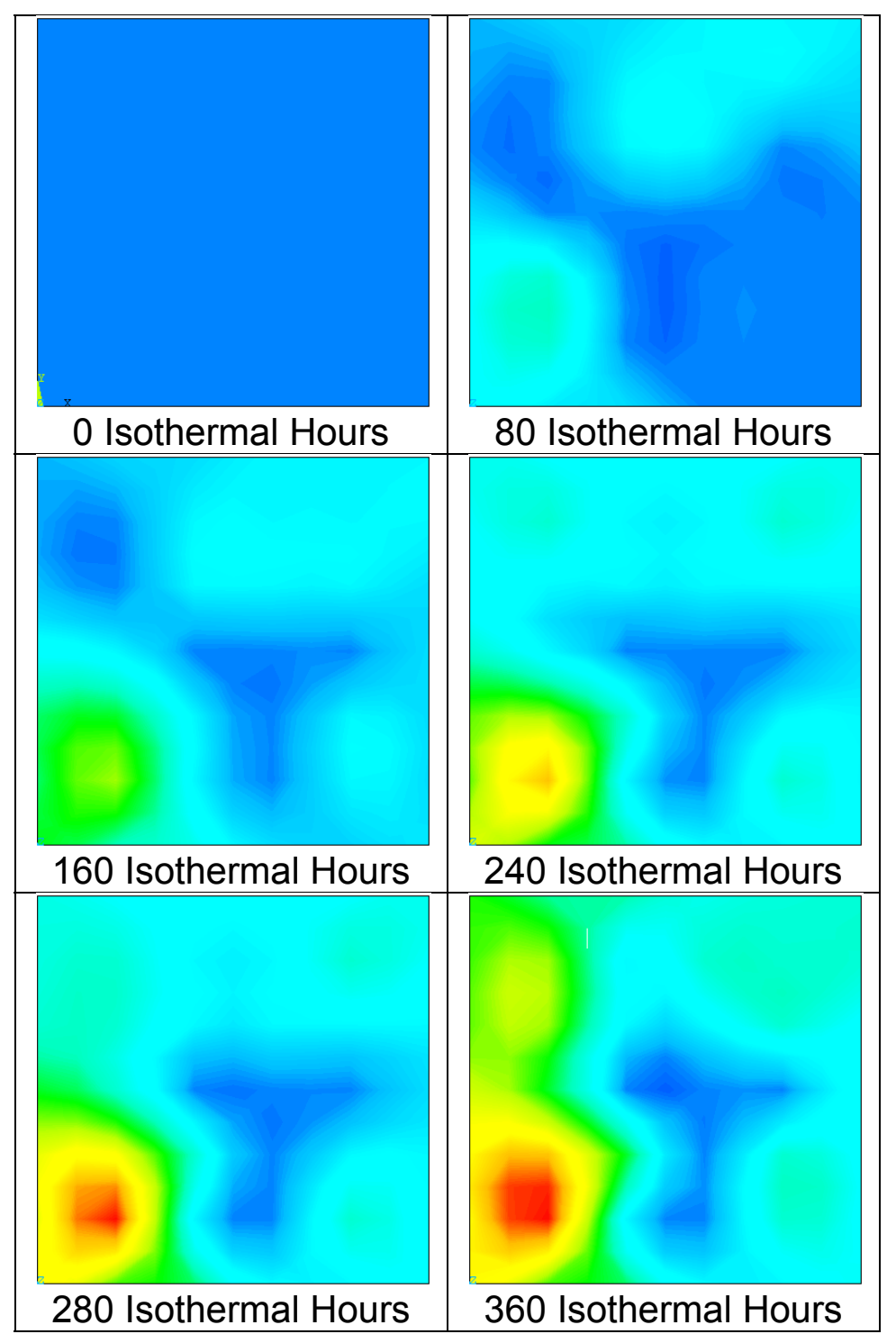

\section{Low Stiffness High Stiffness}

Figure 75: Surface stiffness response color maps for Coupon B obtained after periodical 40 hour isothermal heat treatment cycles at $1100^{\circ} \mathrm{C}$ in an ambient air environment. 

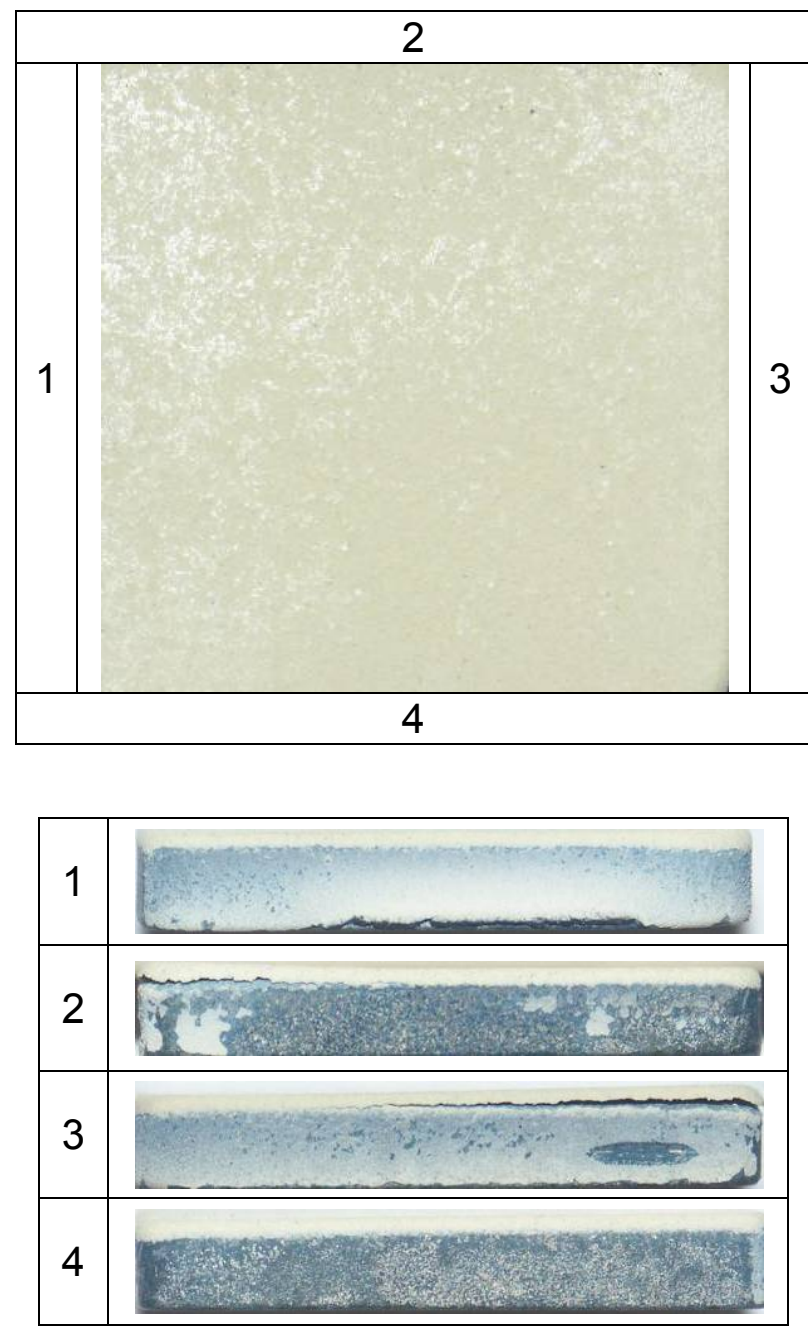

Figure 76: Edge and surface photographs of APS/MCrAIY/RenéN5 Coupon $\mathrm{C}$ after a cumulative 400 isothermal heat treatment hours at $1100^{\circ} \mathrm{C}$ in an ambient air environment and 1.5 weeks storage.

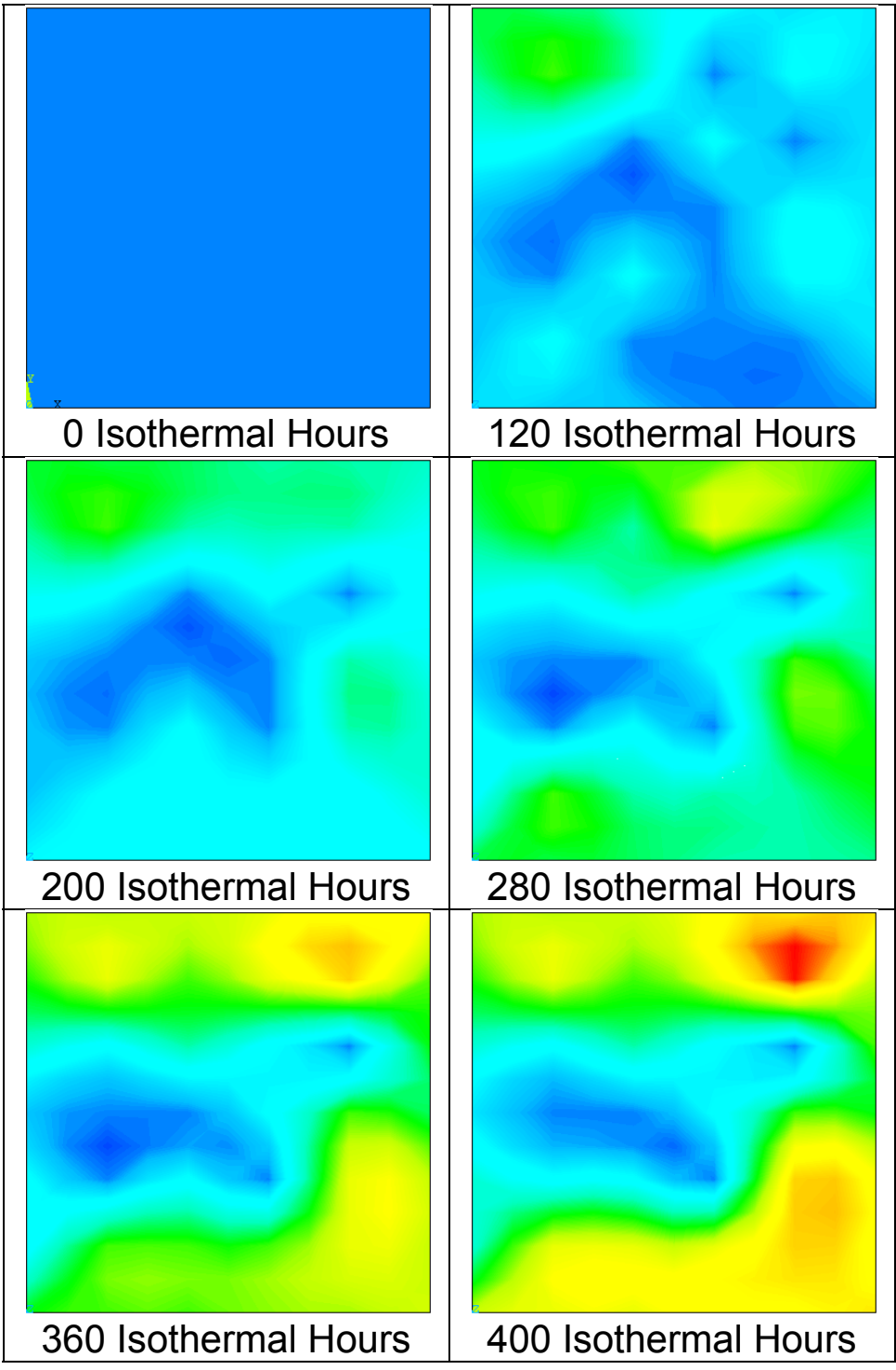

\section{Low Stiffness High Stiffness}

Figure 77: Surface stiffness response color maps for Coupon C obtained after periodical 40 hour isothermal heat treatment cycles at $1100^{\circ} \mathrm{C}$ in an ambient air environment. 

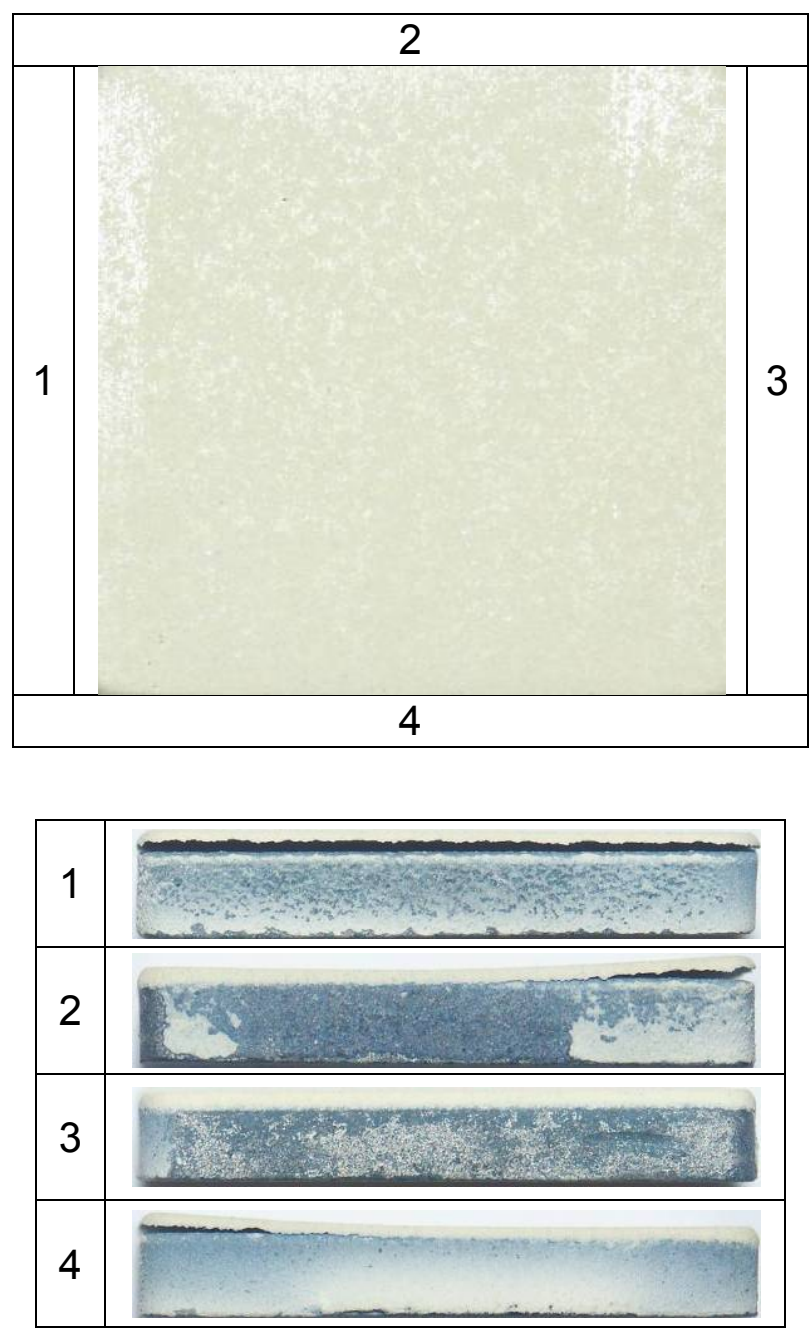

Figure 78: Edge and surface photographs of APS/MCrAIY/RenéN5 Coupon D after cumulative 400 isothermal heat treatment hours at $1100^{\circ} \mathrm{C}$ in an ambient air environment and 1.5 weeks storage.

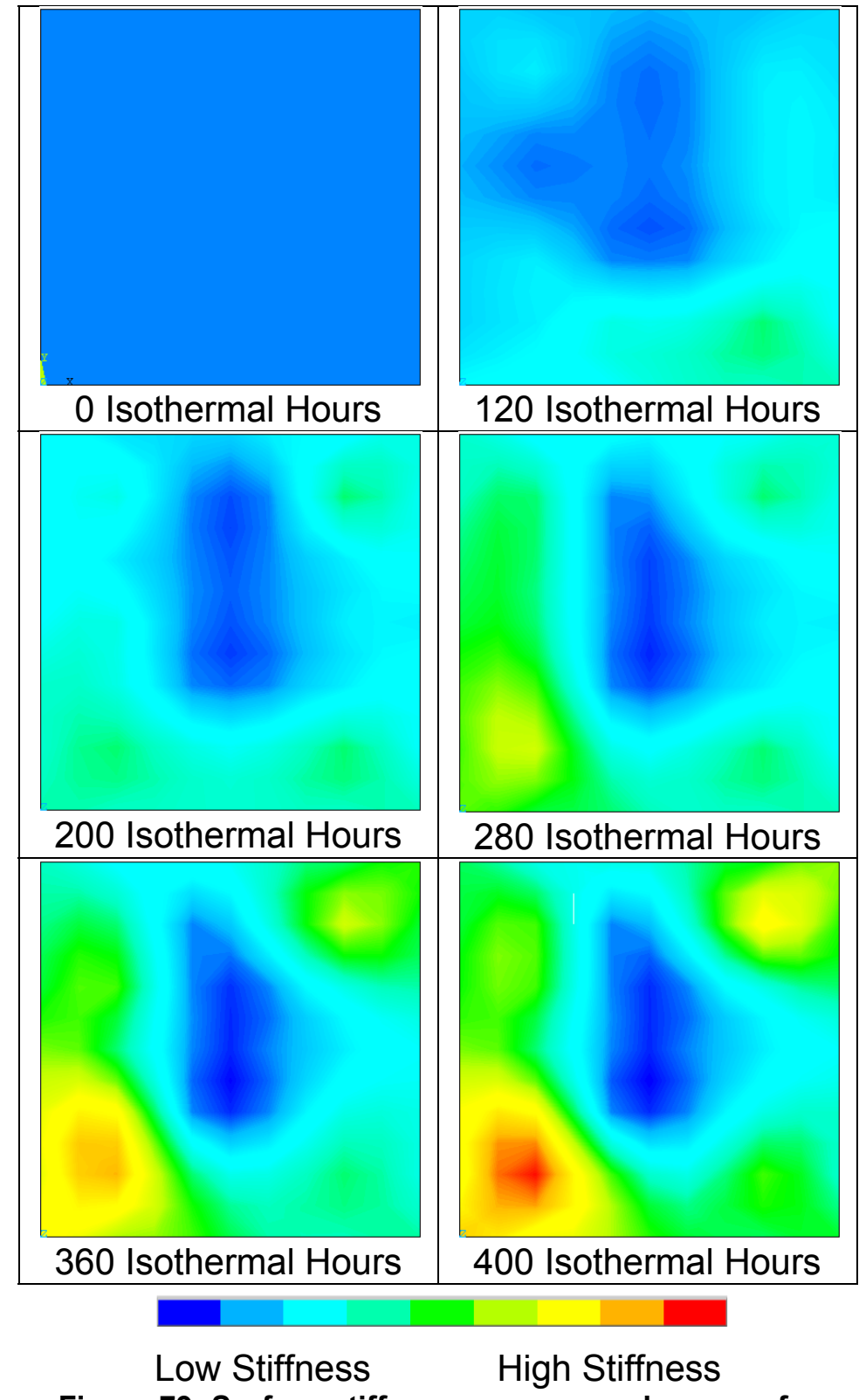

Figure 79: Surface stiffness response color maps for Coupon D obtained after periodical 40 hour isothermal heat treatment cycles at $1100^{\circ} \mathrm{C}$ in an ambient air environment. 

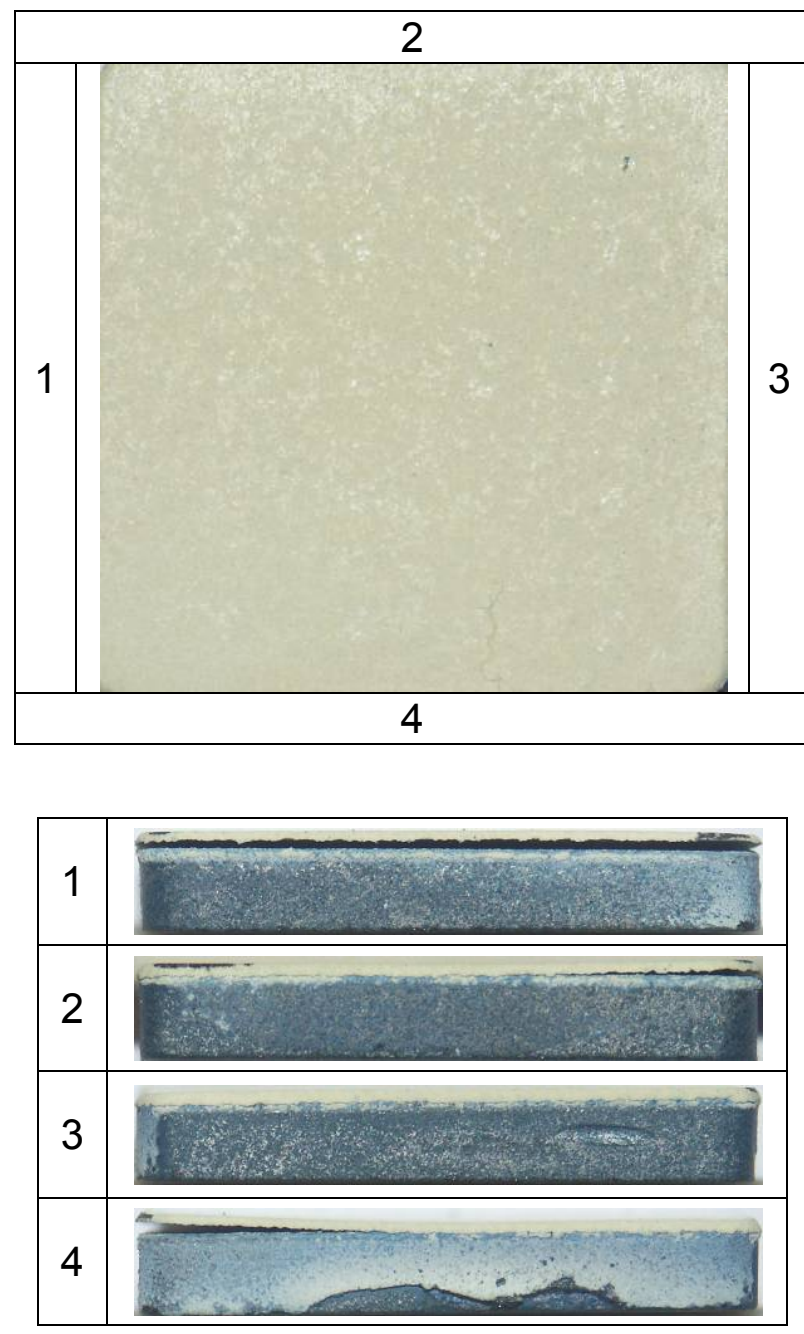

Figure 80: Edge and surface photographs of APS/MCrAIY/RenéN5 Coupon E after cumulative 400 cycles of heat treatment at $1100^{\circ} \mathrm{C}$ in an ambient air environment after 2 days in storage.

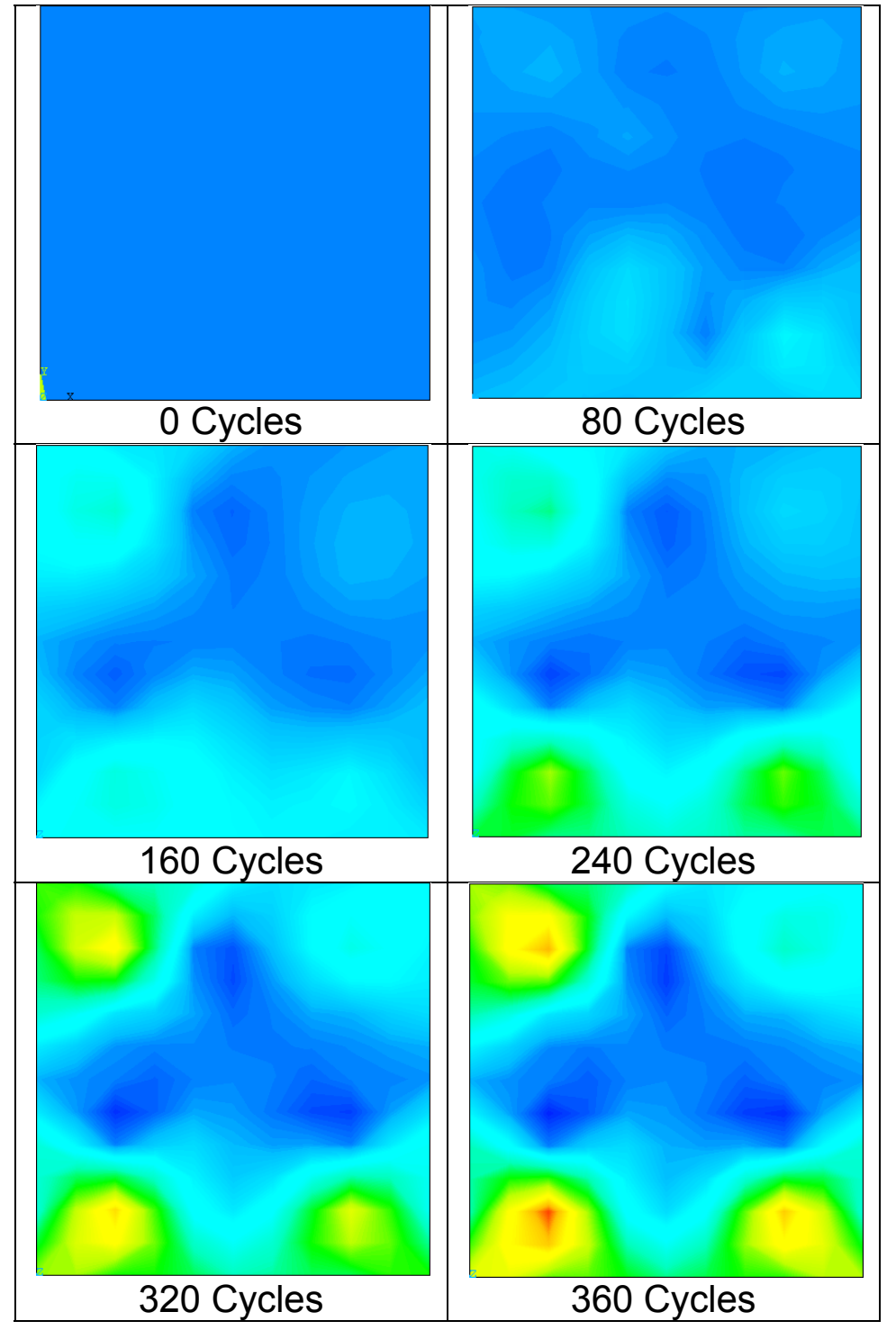

Low Stiffness High Stiffness

Figure 81: Surface stiffness response color maps for Coupon E obtained after periodical 40 cycle heat treatments at $1100^{\circ} \mathrm{C}$ in an ambient air environment. 

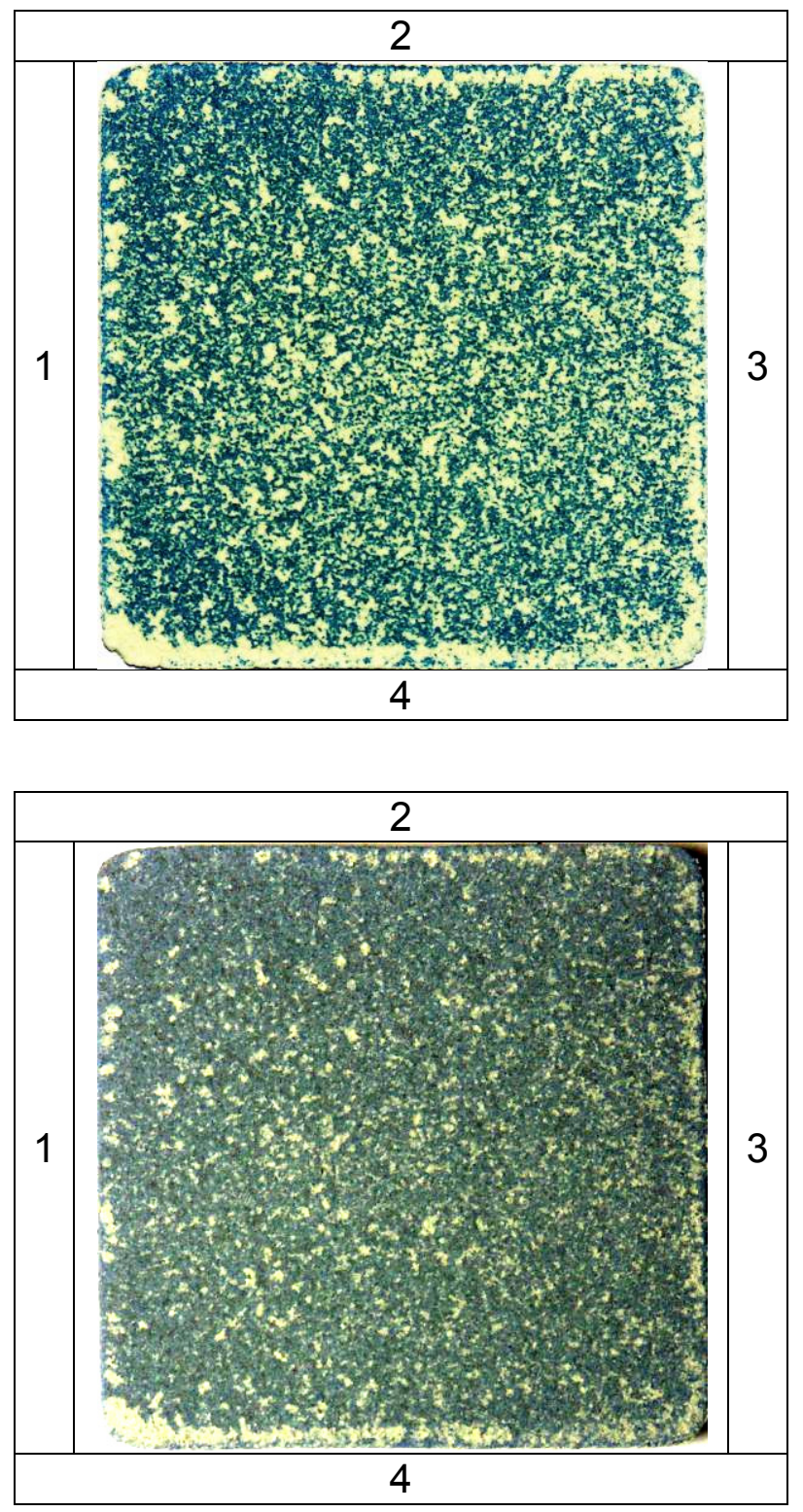

Figure 82: YSZ surface and failure surface photographs of APS/MCrAIY/RenéN5 Coupon F after cumulative 440 cycles of heat treatment at $1100^{\circ} \mathrm{C}$ in an ambient air environment.

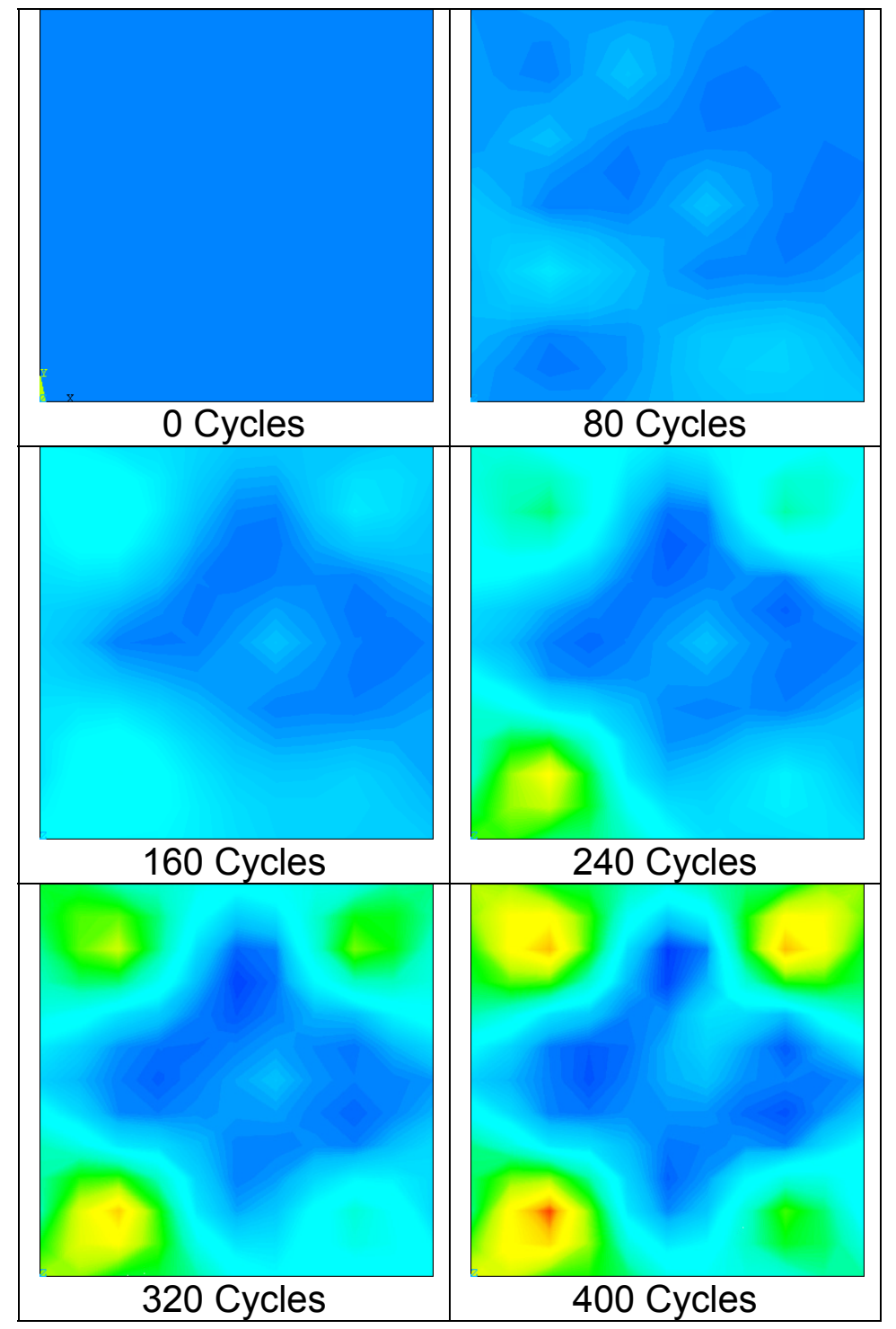

\section{Low Stiffness High Stiffness}

Figure 83: Surface stiffness response color maps for Coupon $F$ obtained after periodical 40 cycle heat treatments at $1100^{\circ} \mathrm{C}$ in an ambient air environment. 

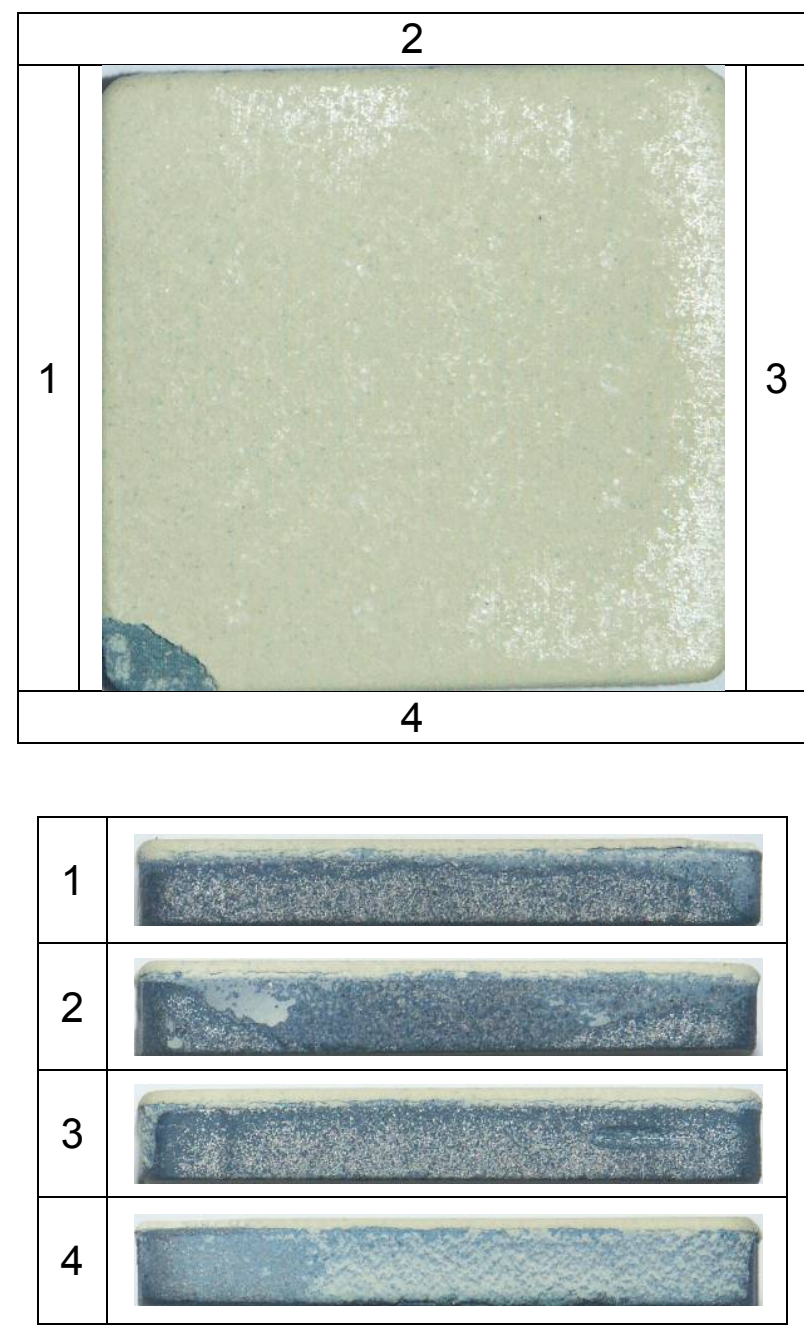

Figure 84: Edge and surface photographs of APS/MCrAIY/RenéN5 Coupon G after cumulative $\mathbf{4 4 0}$ cycles of heat treatment at $1100^{\circ} \mathrm{C}$ in an ambient air environment.

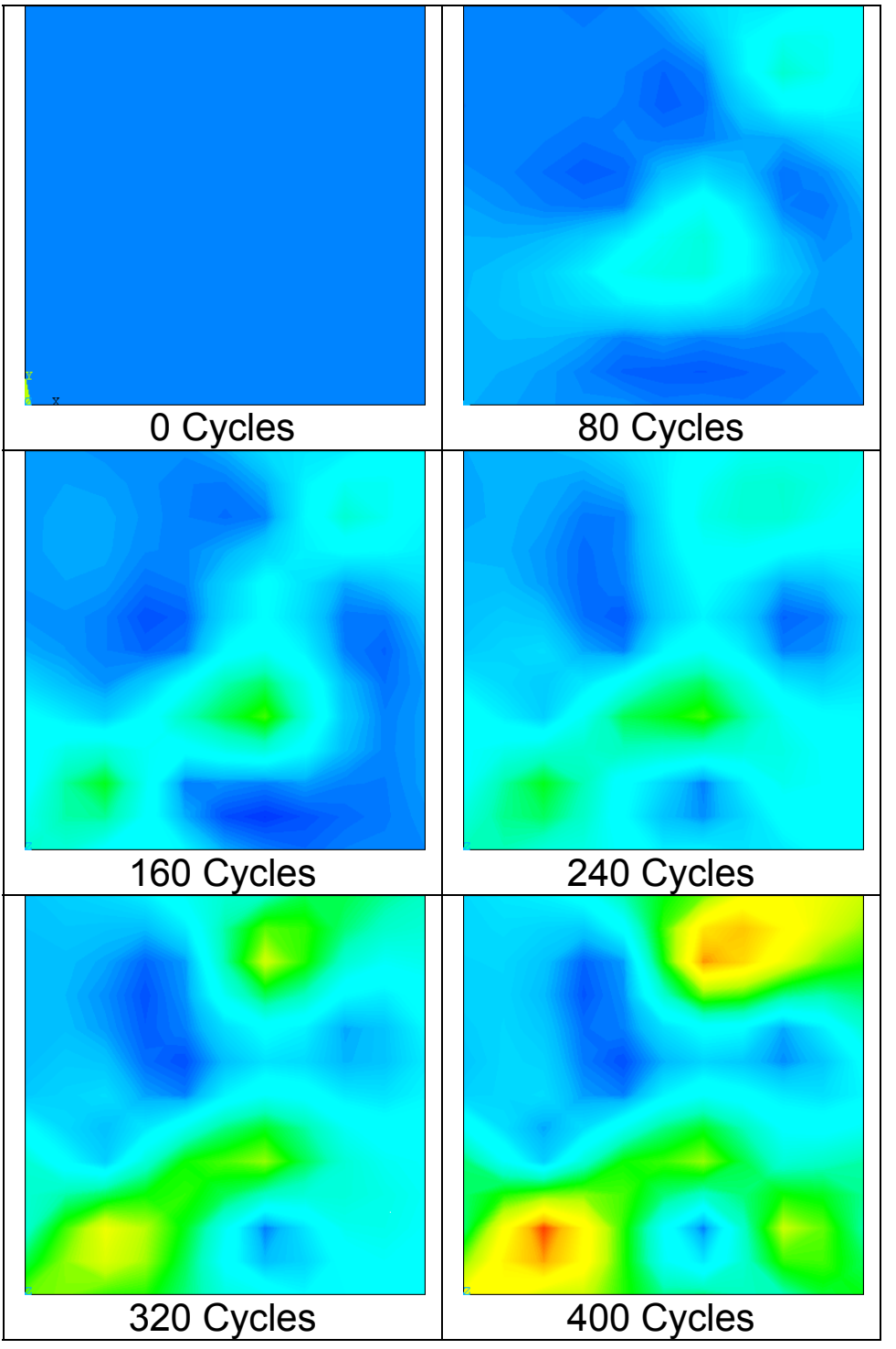

Low Stiffness High Stiffness

Figure 85: Surface stiffness response color maps for Coupon $\mathbf{G}$ obtained after periodical $\mathbf{4 0}$ cycle heat treatments at $1100^{\circ} \mathrm{C}$ in an ambient air environment. 

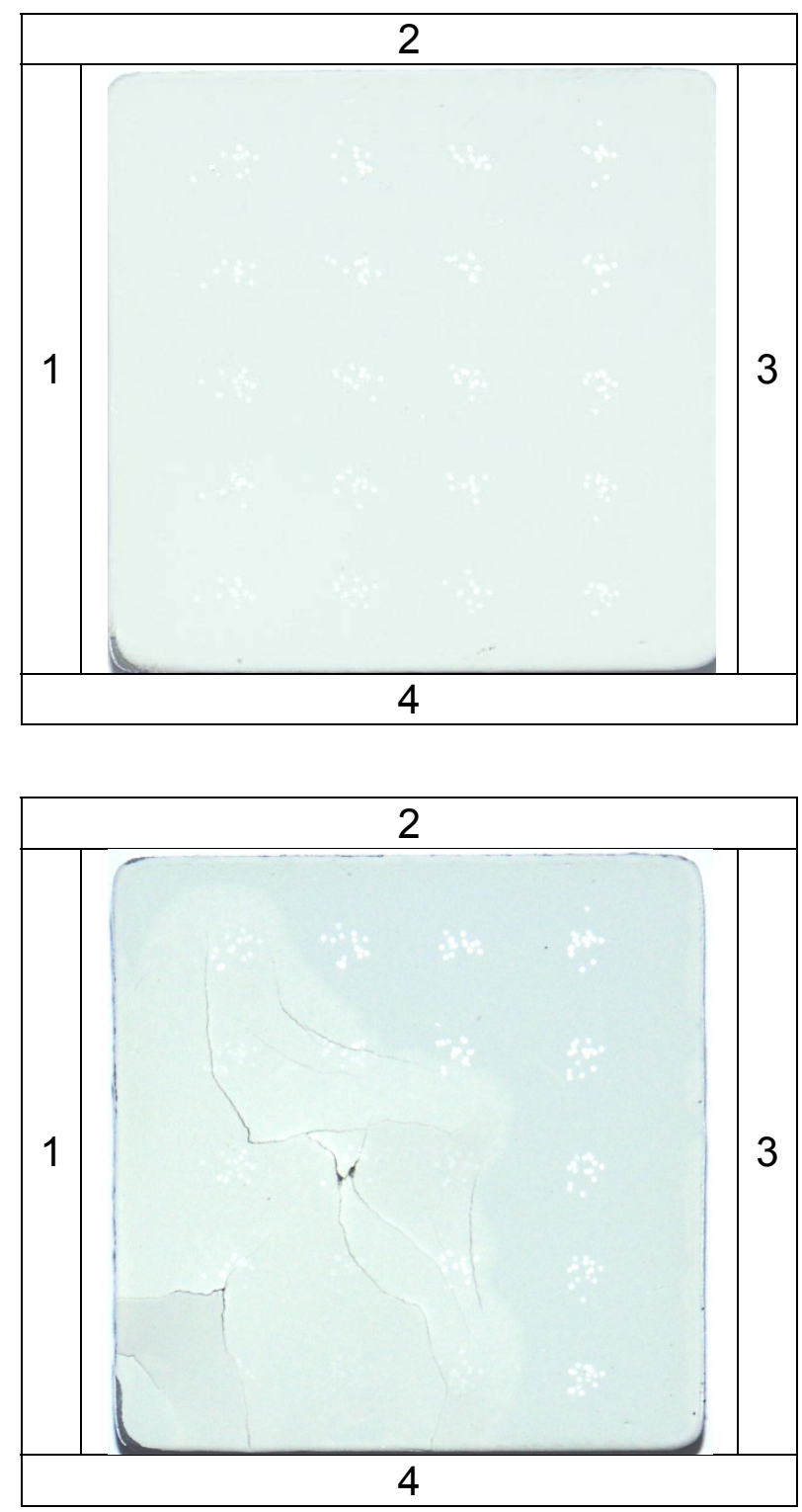

Figure 86: Surface photographs of EBPVD/MDL/RenéN5 Coupon H after a cumulative 640 cycles of heat treatment at $1100^{\circ} \mathrm{C}$ in an ambient air environment.

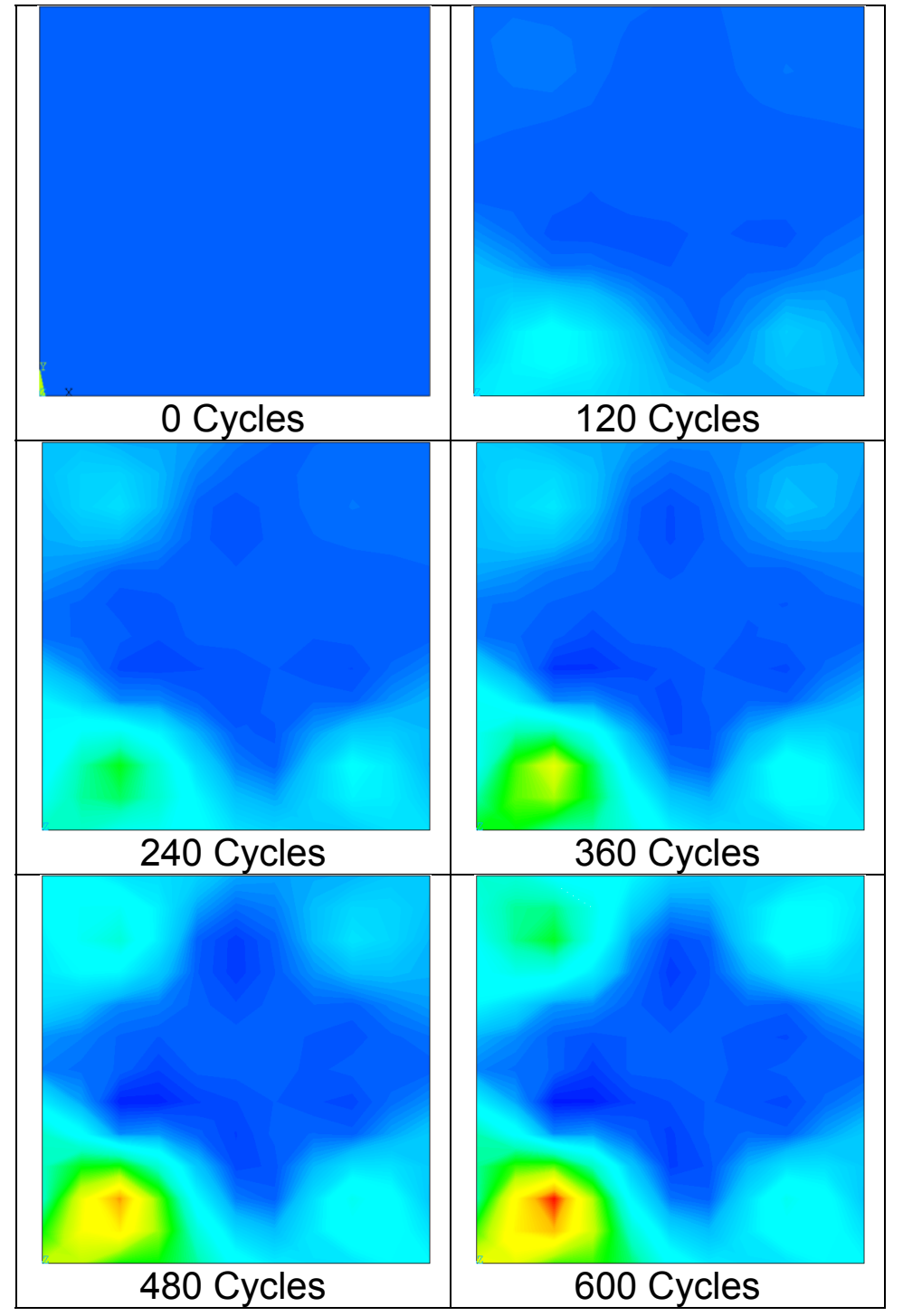

\section{Low Stiffness High Stiffness}

Figure 87: Surface stiffness response color maps for Coupon $\mathrm{H}$ obtained after periodical $\mathbf{4 0}$ cycle heat treatments at $1100^{\circ} \mathrm{C}$ in an ambient air environment. 